\begin{tabular}{|c|c|c|}
\hline \multirow{2}{*}{ Date Submitted: } & WASTE SITE RECLASSIFICATION FORM & \multirow{5}{*}{ Control Number: $2008-028$} \\
\hline & Operable Unit(s): $100-F R+2$ PCK & \\
\hline \multirow{2}{*}{$\begin{array}{l}\text { Originator: J. M. Capron } \\
\text { Phone: } \quad 372-9227\end{array}$} & Waste Site Code: $\quad 120-\mathrm{F}-1$ & \\
\hline & Type of Reclassification Action: & \\
\hline & $\begin{array}{l}\text { Closed Out } \square \text { Interim Closed Out } \otimes \text { No Action } \square \\
\text { RCRA Postclosure } \square \quad \text { Rejected } \square \quad \text { Consolidated } \square\end{array}$ & \\
\hline
\end{tabular}

This form documents agreement among parties listed authorizing classification of the subject unit as Closed Out,. Interim Closed Out, No Action, RCRA Postclosure, Rejected, or Consolidated. This form also authorizes backfill of the waste management unit, if appropriate, for Closed Out and Interim Closed Out units. Final removal from the NPL of No Action and Closed Out waste management units will occur at a future date.

Description of current waste site condition:

The 120-F-1 waste site consisted of two dumping areas located $660 \mathrm{~m}(2,170 \mathrm{ft})$ southeast of the 105-F Reactor containing laboratory equipment and bottles, demolition debris, light bulbs and tubes, small batteries, small drums, and pesticide contaminated soil. It is probable that $108-\mathrm{F}$ was the source of the debris but the material may have come from other locations within the 100-F Area. The site has been remediated and, with regulatory agency concurrence, backfilled. Remediation and verification sampling of this site have been performed in accordance with remedial action objectives and goals established by the Interim Action Record of Decision for the 100-BC-1, 100-BC-2, 100 DR-1, 100-DR-2, 100-FR-1, 100-FR-2, 100-HR-1, 100-HR-2, 100-KR-1, 100-KR-2, 100-IU-2, 100-IU-6, and 200-CW-3 Operable Units, Hanford Site, Benton County, Washington (Remaining Sites ROD), U.S. Environmental Protection Agency, Region 10, Seattle, Washington. The selected action involved: (1) evaluating the site using available process information, (2) remediating the site, (3) demonstrating through verification sampling that cleanup goals have been achieved, and (4) proposing the site for reclassification to Interim Closed Out.

Basis for reclassification:

In accordance with this evaluation, the verification sampling results support a reclassification of this site to Interim Closed Out. The current site conditions achieve the remedial action objectives and the corresponding remedial action goals established in the Remaining Sites ROD. The results of verification sampling show that residual contaminant concentrations do not preclude any future uses (as bounded by the rural-residential scenario) and allow for unrestricted use of shallow-zone soils (i.e., surface to

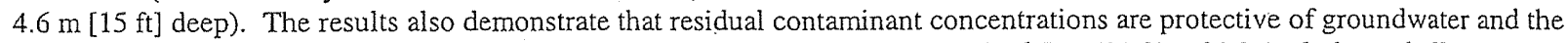
Columbia River. The 120-F-1 excavation has a maximum depth of approximately $6.5 \mathrm{~m}(21 \mathrm{ft})$, which includes a shallow zone and a deep zone. However, the entire excavation area is considered one decision unit, and is closed out using the more restrictive shallow-zone cleanup criteria; therefore, institutional controls to prevent uncontrolled drilling or excavation into the deep zone are not required. The basis for reclassification is described in detail in the Remaining Sites Verification Package for the 120-F-1, Glass Dump Waste Site (attached).

Waste Site Controls:

Engineered Controls: Yes $\square$ No $\square \quad$ Institutional Controls: Yes $\square$ No $\square \quad$ O\&M requirements: Yes $\square$ No $\square$ If any of the Waste Site Controls are checked Yes specify control requirements including reference to the Record of Decision,

R. F. Guercia
DOE Federal Project Director (printed)
Ecology Project Manager (printed)
EPA Project Manager (printed)




\section{REMAINING SITES VERIFICATION PACKAGE FOR THE 120-F-1 GLASS DUMP WASTE SITE}

Attachment to Waste Site Reclassification Form 2008-028

May 2008 


\section{REMAINING SITES VERIFICATION PACKAGE FOR THE 120-F-1 GLASS DUMP WASTE SITE}

\section{EXECUTIVE SUMMARY}

The 120-F-1 waste site is located within the 100-FR-2 Operable Unit on the Hanford Site, approximately $660 \mathrm{~m}(2,170 \mathrm{ft})$ southeast of the $105-\mathrm{F}$ Reactor. The $120-\mathrm{F}-1$ waste site includes two distinct dumping areas.

The 120-F-1 waste site was originally described as a single, open trench filled with fluorescent tubes, incandescent light bulbs, instrument vacuum tubes, small alkaline batteries, chemical bottles and laboratory apparatus with a second area of disturbed soil with surficial plastic debris approximately $46 \mathrm{~m}(150 \mathrm{ft})$ to the southeast (BHI 1994). Due to its proximity, the probable source of the debris found at the 120-F-1 waste site is believed to be the 108-F Building, but the site may have contained debris from other locations within the 100-F Area. Prior to remediation, the original access road was overgrown with $0.9 \mathrm{~m}(3-\mathrm{ft}-)$ high sagebrush, indicating that the site had not been used for many years. The exact dates of site operation are unknown.

The 120-F-1 waste site was interim stabilized March 23, 1998 (BHI 1998a). The trench was covered with 30 sheets of plywood to provide a demarcation layer to aid in future remediation. A protective layer of soil, from material mounded to the side of the trench during the original excavation, was placed over the plywood in a layer approximately 0.2 to $0.6 \mathrm{~m}(0.6$ to $2 \mathrm{ft})$ deep. The site was further protected by a barrier attached to metal posts and warning signs. Samples collected during the 1998 interim stabilization detected contamination of lead and mercury but no radiological contamination (BHI 1998a).

In preparation for remedial action at the site, a standard geophysical investigation was conducted in the vicinity of the glass dump. The second area of debris to the southeast was identified as a waste dumping area in the geophysical interpretation (BHI 2004). The southeastern dumping area proved to be larger than the aforementioned glass dump and contained concrete, wire debris, small drums with heavy oil-type petroleum hydrocarbons, and some stained soil with pesticides (dichlorodiphenyldichloroethylene [DDE] and dichlorodiphenyltrichloroethane [DDT]). The second area was remediated as part of the 120-F-1 Glass Dump Waste Site.

Confirmatory sampling was not performed because the presence of contamination was already documented during interim stabilization. On January 21, 2006, an industrial hygiene investigation of the $120-\mathrm{F}-1$ waste site was conducted to determine whether beryllium was present in the phosphor material used in the fluorescent tubes dumped at the 120-F-1 waste site (BHI 2006). Beryllium was used in the manufacture of fluorescent light bulbs prior to 1949. The previously interim-stabilized waste site was opened using a front-end loader. At this juncture, it was discovered that the plywood barrier had failed and the fluorescent tubes were crushed. Pieces of the fluorescent tubes and accompanying soils were sampled. The samples were analyzed for metals, including mercury and beryllium. Beryllium was detected in one of three samples at a concentration below its average background concentration. It was determined 
that the fluorescent tubes were not manufactured with beryllium and that beryllium would not be a health concern during remove, treat, and dispose (RTD) activities at the 120-F-1 waste site. However, mercury and, to a much lesser extent, metals such as manganese, nickel, and cadmium were detected and determined to be possible airborne inhalation hazards. Therefore, wetting methods for dust suppression were indicated for the pending RTD of the site.

Remedial action at the 120-F-1 waste site began in September 2007 and was completed in March 2008. Two distinct areas were excavated resulting in disposal of approximately 1,505 bank cubic meters of contaminated materials to the Environmental Restoration Disposal Facility.

A summary of the cleanup evaluation for the soil sample results against the applicable criteria is presented in Table ES-1. The results of the verification sampling are used to make reclassification decisions for the 120-F-1 waste site in accordance with the Tri-Party Agreement Handbook Management Procedures, TPA-MP-14 (DOE-RL 2007).

Table ES-1. Summary of Remedial Action Goals for the 120-F-1 Site. (2 Pages)

\begin{tabular}{|c|c|c|c|}
\hline $\begin{array}{l}\text { Regulatory } \\
\text { Requirement }\end{array}$ & Remedial Action Goals & Results & $\begin{array}{l}\text { Remedial Action } \\
\text { Objectives } \\
\text { Attained? }\end{array}$ \\
\hline $\begin{array}{l}\text { Direct Exposure - } \\
\text { Radionuclides }\end{array}$ & $\begin{array}{l}\text { Attain } 15-\mathrm{mrem} / \mathrm{yr} \text { dose rate above } \\
\text { background over } 1,000 \text { years. }\end{array}$ & Radionuclides are not site COPCs. & Yes \\
\hline $\begin{array}{l}\text { Direct Exposure - } \\
\text { Nonradionuclides }\end{array}$ & Attain individual COPC RAGs. & $\begin{array}{l}\text { All individual COPC concentrations } \\
\text { are below the direct exposure criteria. }\end{array}$ & Yes \\
\hline \multirow[t]{4}{*}{$\begin{array}{l}\text { Risk Requirements - } \\
\text { Nonradionuclides }\end{array}$} & $\begin{array}{l}\text { Attain a hazard quotient of }<1 \text { for } \\
\text { all individual noncarcinogens. }\end{array}$ & $\begin{array}{l}\text { All individual hazard quotients are less } \\
\text { than } 1 .\end{array}$ & \multirow{4}{*}{ Yes } \\
\hline & $\begin{array}{l}\text { Attain a cumulative hazard } \\
\text { quotient of }<1 \text { for noncarcinogens. }\end{array}$ & $\begin{array}{l}\text { The cumulative hazard quotient }(4.2 \times \\
\left.10^{-2}\right) \text { is less than } 1 .\end{array}$ & \\
\hline & $\begin{array}{l}\text { Attain an excess cancer risk of } \\
<1 \times 10^{-6} \text { for individual } \\
\text { carcinogens. }\end{array}$ & $\begin{array}{l}\text { The individual excess cancer risk for } \\
\text { carcinogens are less than } 1 \times 10^{-6} \text {. }\end{array}$ & \\
\hline & $\begin{array}{l}\text { Attain a cumulative excess cancer } \\
\text { risk of }<1 \times 10^{-5} \text { for carcinogens. }\end{array}$ & $\begin{array}{l}\text { The total excess cancer risk } \\
\left(1.1 \times 10^{-6}\right) \text { is less than } 1 \times 10^{-5} \text {. }\end{array}$ & \\
\hline \multirow{4}{*}{$\begin{array}{l}\text { Groundwater/River } \\
\text { Protection - } \\
\text { Radionuclides }\end{array}$} & $\begin{array}{l}\text { Attain single-COPC groundwater } \\
\text { and river protection RAGs. }\end{array}$ & \multirow{4}{*}{ Radionuclides are not site COPCs. } & \multirow{4}{*}{ Yes } \\
\hline & $\begin{array}{l}\text { Attain national primary drinking } \\
\text { water standards: } 4 \text { mrem/yr } \\
\text { (beta/gamma) dose rate to target } \\
\text { receptor/organs. }\end{array}$ & & \\
\hline & $\begin{array}{l}\text { Meet drinking water standards for } \\
\text { alpha emitters: the most stringent } \\
\text { of } 15 \mathrm{pCi} / \mathrm{L} \mathrm{MCL} \text { or } 1 / 25 \text { th of the } \\
\text { derived concentration guides from } \\
\text { DOE Order } 5400.5^{b}\end{array}$ & & \\
\hline & $\begin{array}{l}\text { Meet total uranium standard of } \\
30 \mu \mathrm{g} / \mathrm{L}(21.2 \mathrm{pCi} / \mathrm{L})\end{array}$ & & \\
\hline
\end{tabular}


Table ES-1. Summary of Remedial Action Goals for the 120-F-1 Site. (2 Pages)

\begin{tabular}{|l|l|l|l|}
\hline \multicolumn{1}{|c|}{$\begin{array}{c}\text { Regulatory } \\
\text { Requirement }\end{array}$} & \multicolumn{1}{|c|}{ Remedial Action Goals } & Results & $\begin{array}{c}\text { Remedial Action } \\
\text { Objectives } \\
\text { Attained? }\end{array}$ \\
\hline $\begin{array}{l}\text { Groundwater/River } \\
\text { Protection - } \\
\text { Nonradionuclides }\end{array}$ & $\begin{array}{l}\text { Attain individual nonradionuclide } \\
\text { groundwater and river cleanup } \\
\text { requirements. }\end{array}$ & $\begin{array}{l}\text { Residual concentrations of selenium, } \\
\text { diesel range TPH, and motor oil range } \\
\text { TPH are above the groundwater and } \\
\text { river protection soil RAGs. However, } \\
\text { RESRAD modeling predicts these } \\
\text { constituents will not reach } \\
\text { groundwater (and, therefore, the } \\
\text { Columbia River) within 1,000 years. }{ }^{\text {d }}\end{array}$ & Yes \\
\hline
\end{tabular}

a "National Primary Drinking Water Regulations" (40 Code of Federal Regulations 141).

${ }^{\mathrm{b}}$ Radiation Protection of the Public and the Environment (DOE Order 5400.5).

c Remedial Design Report/Remedial Action Work Plan for the 100 Area (DOE-RL 2005b).

d Based on the 100 Area Analogous Sites RESRAD Calculations (BHI 2005), these constituents are not predicted to migrate more than $2 \mathrm{~m}(6.6 \mathrm{ft}$ ) vertically in 1,000 years (based on the lowest soil-partitioning coefficient distribution [for TPH] of $50 \mathrm{~mL} / \mathrm{g}$ ).

$\mathrm{COPC}=$ contaminant of potential concern

$\mathrm{MCL}=$ maximum contaminant level (drinking water standard)

$\mathrm{RAG} \quad=$ remedial action goal

RESRAD $=$ RESidual RADioactivity (dose model)

$\mathrm{TPH}=$ total petroleum hydrocarbons

Verification sampling for both the northwest and the southeast excavations within the 120-F-1 waste site was performed in December 2007 (WCH 2007b) to determine if the remedial action goals had been met. The contaminants of potential concern (COPCs) for verification sampling included inductively coupled plasma metals, hexavalent chromium, mercury, semivolatile organic compounds, and polychlorinated biphenyls (PCBs) (WCH 2007a). In the southeast excavation, total petroleum hydrocarbons were also COPCs.

Several iterations of material removal and sampling for limited analytes were conducted in the northwest excavation after the initial verification sampling results showed elevated contaminant levels. The verification sample results from the northwest excavation showed elevated levels of several pesticides and metals. Additional material was removed and the boundary of the waste site was increased. A second sample design was prepared for the northwest portion, and this area was sampled for PCBs and pesticides on February 4, 2008. The second set of samples also showed elevated levels of pesticides. Again, material was removed and the site boundary was increased. The northwest excavation was then sampled for pesticide analytes only on February 19,2008 , using a third, revised sample design. Slightly elevated pesticide levels were still present and additional material was removed from the area, which again changed the remediation boundary. Sampling for pesticide analytes was performed on March 5, 2008 in the northwest excavation using a fourth sample design. The fourth set of sampling results showed detectable levels of pesticides at a single sample site. A final remediation was performed at this specific area after which the same location was resampled. Pesticides were undetected in the sample. The full set of verification samples for the northwest excavation was then taken on March 18, 2008 , using the sampling coordinates from the fourth sample design.

In accordance with this evaluation, the verification sampling results support a reclassification of this site to Interim Closed Out. The current site conditions achieve the remedial action 
objectives and the corresponding remedial action goals established in the Remedial Design Report/Remedial Action Work Plan for the 100 Area (DOE-RL 2005b) and the Interim Action Record of Decision for the 100-BC-1, 100-BC-2, 100-DR-1, 100-DR-2, 100-FR-1, 100-FR-2, 100-HR-1, 100-HR-2, 100-KR-1, 100-KR-2, 100-IU-2, 100-IU-6, and 200-CW-3 Operable Units, Hanford Site, Benton County, Washington (EPA 1999). These results show that residual soil concentrations support future land uses that can be represented (or bounded) by a rural-residential scenario. The results also demonstrate that residual contaminant concentrations

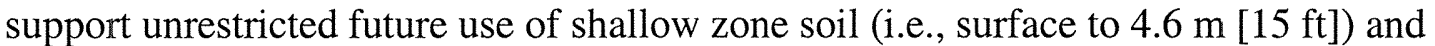
contaminant levels remaining in the soil are protective of groundwater and the Columbia River. Although a portion of the excavation extends into the deep zone, the site is being closed out using shallow zone criteria; therefore, no deep zone institutional controls are required.

A comparison against ecological risk screening levels has been made for the site contaminants of potential concern (COPCs) and other constituents. Screening levels were not exceeded for the site constituents, with the exception of antimony, boron, manganese, mercury, and vanadium. Exceedance of screening values does not necessarily indicate the existence of risk to ecological receptors. It is believed that the presence of these constituents does not pose a risk to ecological receptors because concentrations of antimony, manganese, and vanadium are below site background levels, and boron concentrations are consistent with those seen elsewhere at the Hanford Site (no established background value is available for boron). A single verification sample contained a concentration of mercury approximately two times above Hanford Site background. All other samples of mercury are below the ecological screening levels. 


\section{REMAINING SITES VERIFICATION PACKAGE FOR THE 120-F-1 GLASS DUMP WASTE SITE}

\section{STATEMENT OF PROTECTIVENESS}

This report demonstrates that the 120-F-1 glass dump waste site meets the objectives for interim closure as established in the Remedial Design Report/Remedial Action Work Plan for the 100 Area (RDR/RAWP) (DOE-RL 2005b) and the Interim Action Record of Decision for the 100-BC-1, 100-BC-2, 100-DR-1, 100-DR-2, 100-FR-1, 100-FR-2, 100-HR-1, 100-HR-2, 100-KR-1, 100-KR-2, 100-IU-2, 100-IU-6, and 200-CW-3 Operable Units, Hanford Site, Benton County, Washington (Remaining Sites ROD) (EPA 1999). These results show that residual soil concentrations support future land uses that can be represented (or bounded) by a rural-residential scenario. The results also demonstrate that residual contaminant concentrations

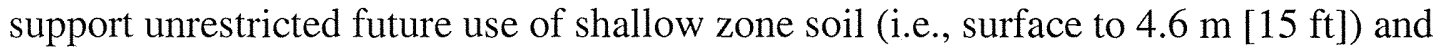
contaminant levels remaining in the soil are protective of groundwater and the Columbia River. Although a portion of the excavation extends into the deep zone, the site is being closed out using shallow zone criteria; therefore, no deep zone institutional controls are required.

A comparison against ecological risk screening levels has been made for the site contaminants of potential concern (COPCs) and other constituents. Screening levels were not exceeded for the site constituents, with the exception of antimony, boron, manganese, mercury, and vanadium. Exceedance of screening values does not necessarily indicate the existence of risk to ecological receptors. It is believed that the presence of these constituents does not pose a risk to ecological receptors because concentrations of antimony, manganese, and vanadium are below site background levels, and boron concentrations are consistent with those seen elsewhere at the Hanford Site (no established background value is available for boron). A single verification sample contained a concentration of mercury approximately two times above Hanford Site background. All other samples of mercury are below the ecological screening levels.

\section{GENERAL SITE INFORMATION AND BACKGROUND}

The 120-F-1 glass dump waste site, part of the 100-FR-2 Operable Unit, was located approximately $660 \mathrm{~m}(2,165 \mathrm{ft}$ ) southeast of the 105-F Reactor (Figure 1$)$. The site originally consisted of an uncovered trench filled with waste (northwest excavation), but a second dumping area (southeast excavation) was later added as described below.

The 120-F-1 waste site was designated in the RDR/RAWP (DOE-RL 2005b) and the Remaining Sites ROD (EPA 1999) as an area for remove/treat/dispose (RTD) due to reports of fluorescent tubes, vacuum tubes, small batteries, and empty chemical bottles in an open trench. In accordance with the Sampling and Analysis Instruction for the 120-F-1 Glass Dump Site (BHI 1998b), the site was surveyed for volatile organic compounds and mercury vapors. No 
Figure 1. 120-F-1 Waste Site Location Map.

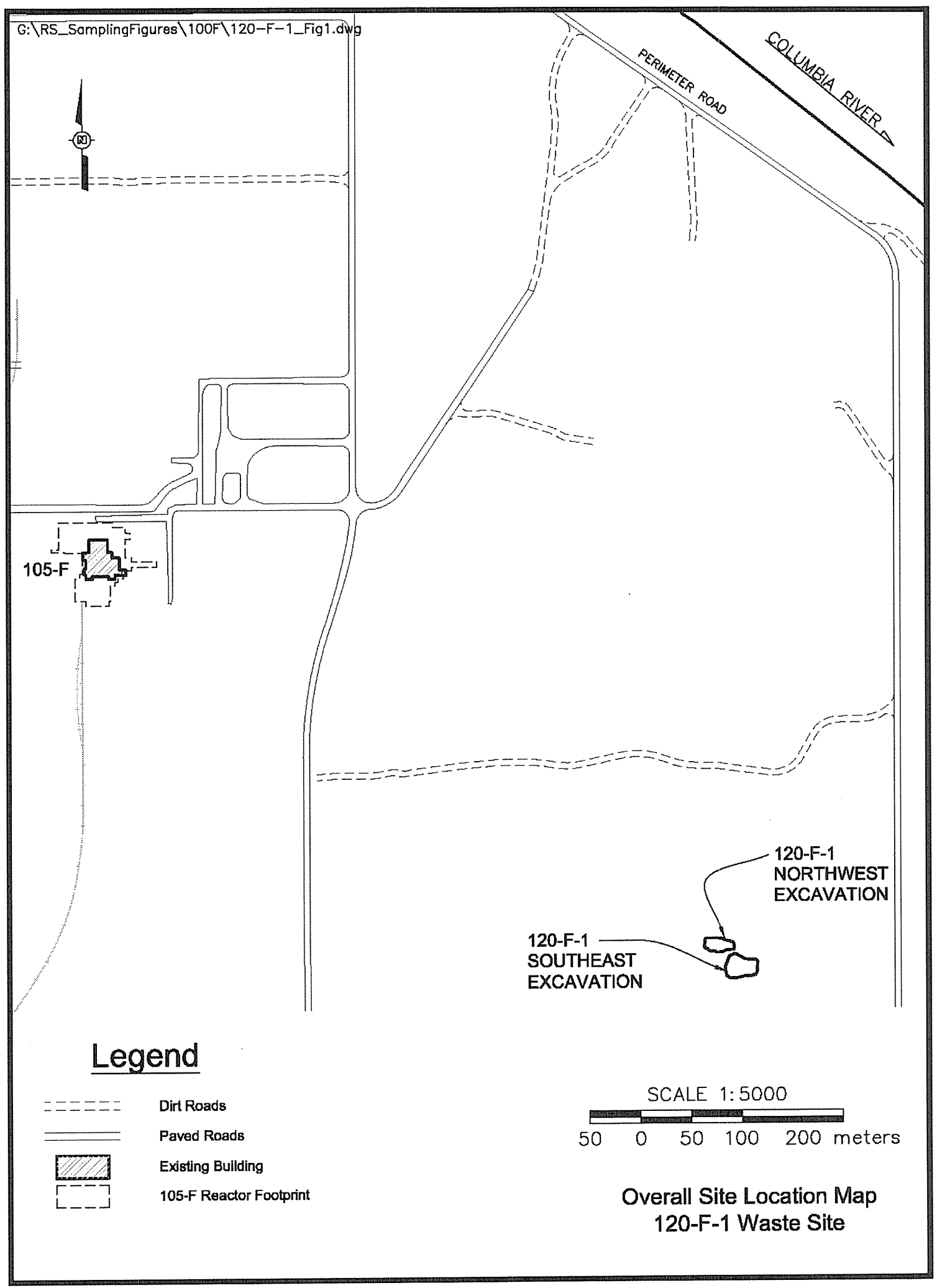


organic vapors were detected, but mercury vapors were detected in the immediate vicinity of the broken fluorescent tubes.

Interim stabilization and limited sampling was performed on March 23, 1998. 'Interim stabilization was conducted as a housekeeping activity to reduce and stabilize hazards and to deter accidental or inadvertent entry. Evaluation of the data from samples collected during interim stabilization is provided in the 120-F-1 Glass Dump Interim Stabilization Final Report (BHI 1998a). The data evaluation concluded that no radiological contamination was present and the lead and mercury results associated with the fluorescent bulbs reaffirmed that RTD was required at the $120-\mathrm{F}-1$ waste site.

\section{PRE-REMEDIATION ACTIVITIES}

On January 21, 2006, an industrial hygiene investigation of the 120-F-1 waste site was conducted to determine whether beryllium was present in the phosphor material used in the fluorescent tubes dumped at the 120-F-1 waste site (BHI 2006). Beryllium was used in the manufacture of fluorescent light bulbs prior to 1949. The previously interim-stabilized waste site was opened using a front-end loader. At this juncture, it was discovered that the plywood barrier had failed and the fluorescent tubes were crushed. Pieces of the fluorescent tubes and accompanying soils were sampled. The samples were analyzed for metals, including mercury and beryllium. Beryllium was detected in one of three samples at a concentration below its average background concentration. It was determined that the fluorescent tubes were not manufactured with beryllium and that beryllium would not be a health concern during RTD of the 120-F-1 waste site. However, mercury and, to a much lesser extent, metals such as manganese, nickel, and cadmium were detected and determined to be possible airborne inhalation hazards. Therefore, wetting methods for dust suppression were indicated for the pending RTD of the site.

\section{Nonintrusive Investigation Results}

In preparation for remedial action at the site, a standard geophysical investigation was conducted in the vicinity of the glass dump (BHI 2004). The second area of debris was identified in the geophysical interpretation, just southeast of the original glass dump. The second, southeast dumping area was larger than the original, northwest glass dump and contained concrete, wire debris, small drums with heavy oil-type petroleum hydrocarbons, and stained soil with pesticides (dichlorodiphenyldichloroethylene [DDE] and dichlorodiphenyltrichloroethane [DDT] ).

\section{Confirmatory Sample Design}

The 120-F-1 site was sent directly to remediation without confirmatory sampling based on process knowledge and sampling results (BHI 1994, 1998a). 


\section{REMEDIAL ACTION SUMMARY}

Remediation of the 120-F-1 waste site was performed during September 2007. Approximately 1,505 bank cubic meters (BCM) of debris from both of the 120-F-1 dump sites was excavated and disposed of at the Environmental Restoration Disposal Facility (ERDF). Eight in-process samples were collected prior to the initial verification sampling (Appendix A). From the northwest excavation, one sample of suspect asbestos-containing material (ACM) (J152H5), one sample of the soil beneath the ACM (J152H6), and two additional soil samples (J153H3 and J153H4). From the southeast excavation, in process samples were collected from an ERDF container with oil saturated soil (J152V6), the site of the excavated soil (J155N6), a site with yellow staining before excavation (J15JB0) and after excavation (J15P45). Remedial action continued in the northwest excavation even after verification sampling due to elevated levels of pesticides in the samples. A final post-excavation civil survey of the waste site is presented in Figure 2.

The northwest excavation contained fluorescent light bulbs and laboratory glassware, as well as most of the pesticide contaminated soil from the waste site. The southeast excavation contained a significant amount of demolition debris such as concrete, wire, and steel, as well as breached oil drums and pesticide contaminated soil. The southeast excavation is deeper than the northwest excavation because of the buried debris and stained soil. Selected photos of the material found at the 120-F-1 waste site are presented in Appendix B.

\section{VERIFICATION SAMPLING ACTIVITIES}

Remedial action goals (RAGs) are the specific numeric goals against which the cleanup verification data are evaluated to demonstrate attainment of the remedial action objectives for the site. Verification sampling for the 120-F-1 waste site was initially performed on December 3 and December 17, 2007 (WCH 2007b) to collect data from both excavations to determine if the RAGs had been met. Inadvertently, hexavalent chromium analysis for these samples was not requested while analyses for anions, cyanide, and sulfide were added. Several iterations of material removal and sampling for limited analytes were conducted in the northwest excavation after the initial verification sampling results showed elevated contaminant levels (Appendix A). The verification sample design for the northwest area was updated to account for changes in the excavation boundary. The final verification sampling for the northwest excavation was conducted on March 18, 2008. Hexavalent chromium sampling for the southeast excavation was performed on February 19, 2008. The following subsections provide additional discussion of the information used to develop the verification sampling design. The results of verification sampling are also summarized to support interim closure of the site.

\section{Contaminants of Potential Concern}

The waste site COPCs for the 120-F-1 waste site are described in the verification work instruction (WCH 2007a). COPCs for verification sampling in the northwestern excavation are inductively coupled plasma (ICP) metals, hexavalent chromium, mercury, semivolatile organic compounds by semivolatile organic analysis, pesticides, and polychlorinated biphenyls (PCBs). 
Figure 2. Post-Excavation Civil Survey of the 120-F-1 Waste Site.

$G: \backslash 100 F \backslash 060208 A . d w g$

- (10)



SCALE 1:500

$50010 \quad 20$ meters
120-F-1 Waste Site
Post-Excavation
Topography Map


In the southeast excavation, the COPCs are the same as in the northwest excavation with the addition of total petroleum hydrocarbons. All analyses are discussed in the Data Evaluation portion of this remaining sites verification package.

\section{Verification Sample Design}

This section describes the basis for selection of an appropriate sample design and determination of the number of verification samples that were collected. The 120-F-1 waste site was divided into two decision units for the purpose of verification sampling. The first decision unit consisted of the southeast excavation and the second decision unit consisted of the northwest excavation.

\section{Verification Sampling - Excavation Footprint}

The decision rule for demonstrating compliance with the cleanup criteria requires comparison of the true population mean, as estimated by the $95 \%$ upper confidence limit (UCL) on the sample mean, with the cleanup level. Therefore, a statistical sampling design was the preferred verification sampling approach for this site because the distribution of potential residual soil contamination over the site was uncertain. The Washington State Department of Ecology publication, Guidance on Sampling and Data Analysis Methods (Ecology 1995), recommends that systematic sampling with sample locations distributed over the entire study area be used. This sampling approach is referred to by the Washington State Department of Ecology as "areawide sampling."

Statistical parameters (i.e., standard deviation within the populations) for residual contaminant levels following remediation at the 120-F-1 waste site were unknown at the time of sample design development. Therefore, the standard deviation of the residual contaminant population was assumed to be less than $25 \%$ of the corresponding decision thresholds for the population. This assumption was verified later using the resulting verification sampling data in Appendix C. The assumption held true for all analytes with the exception of lead in the southeast excavation. This topic will be considered in the data quality assessment for the data set.

Each excavation footprint was delineated in Visual Sample Plan ${ }^{1}$ and used as the basis for location of a random-start systematic grid for verification soil sample collection locations. A total of 10 verification soil samples were calculated to be collected on a random-start, triangular grid for each sampling area. Because the nature of the debris found at the two dumping areas within the 120-F-1 waste site was significantly different and because the COPCs for the two areas are different, each area will be separately evaluated with 10 samples collected within each area. A triangular grid was selected for this investigation based on studies that indicate triangular grids are superior to square grids (Gilbert 1987). Additional discussion of the development of the statistical verification design is available in the $120-\mathrm{F}-1$ verification work instruction (WCH 2007a).

Verification samples from both excavations were collected in December 2007 for all COPCs except for hexavalent chromium. In its place, IC anions, cyanide, and sulfide analyses were inadvertently requested. The sample results showed elevated levels of several pesticides

\footnotetext{
${ }^{1}$ Visual Sample Plan is a site map-based user-interface program that may be downloaded at http://vsp.pnl.gov.
} 
(chlordane, heptachlor, dieldrin, endrin, and DDD) and had a higher than acceptable practical quantitation limit for several PCB samples from the northwest excavation (Appendix A, Table A-2). Additional material was removed from the northwest excavation, and a second sample design for the northwest excavation only was developed due to an increase in the excavation boundary. Samples were collected on February 4, 2008, for PCB and pesticide analyses only. The results of these samples showed several elevated pesticide values (Appendix A, Table A-3), and additional material was removed from the northwest excavation. The excavation boundary was again expanded and an updated sample design was prepared. Samples were collected from these locations on February 19, 2008. Sample results for 3 of the 10 sample locations showed slightly elevated levels of one or more pesticides (Appendix A, Table A-4). Additional material was again removed and samples were collected at locations from a new sample design on March 5, 2008. Only one sample (NW-5) showed detection of pesticides (Appendix A, Table A-5). Additional material was removed from this location, but the excavation boundary did not change. This single sample location was resampled on March 11, 2008, with no detected pesticides (Appendix A, Table A-6). With regulatory agency concurrence (WCH 2008a), this final sampling design was then used to collect verification samples from all 10 locations in the northwest excavation for the full suite of analyses. The revised sample design for the northwest excavation, presented in Appendix D, differed from that in the original design (WCH 2007a) only in the specific sample locations due to the enlarged waste site boundary. The statistical assumptions and parameters were not altered in the design revision.

Summaries of the samples collected and the analyses performed for the verification sampling event are presented in Table 1 and the locations are shown in Figure 3. All sampling was performed in accordance with ENV-1, Environmental Monitoring \& Management, to fulfill the requirements of the 100 Area Remedial Action Sampling and Analysis Plan (DOE-RL 2005a).

Table 1. Verification Sample Summary for the 120-F-1 Waste Site." (3 Pages)

\begin{tabular}{|c|c|c|c|c|}
\hline \multirow{2}{*}{$\begin{array}{l}\text { Sample } \\
\text { Location }\end{array}$} & \multirow{2}{*}{$\begin{array}{l}\text { Sample } \\
\text { Media }\end{array}$} & $\begin{array}{c}\text { Actual } \\
\text { Coordinates }^{b}\end{array}$ & \multirow{2}{*}{$\begin{array}{l}\text { HEIS } \\
\text { Number }\end{array}$} & \multirow{2}{*}{ Sample Analysis $^{c}$} \\
\hline & & $\begin{array}{l}\text { Northing } \\
\text { Easting }\end{array}$ & & \\
\hline $\mathrm{SE}-1$ & Soil & $\begin{array}{l}\text { N } 147190.5 \\
\text { E } 581051.3\end{array}$ & $\mathrm{~J} 16332$ & $\begin{array}{l}\text { ICP metals, mercury, SVOA, PCBs, pesticides, } \\
\text { TPH, IC anions, total cyanide, sulfides }\end{array}$ \\
\hline $\mathrm{SE}-2$ & Soil & $\begin{array}{l}\text { N } 147185.4 \\
\text { E } 581057.7\end{array}$ & $\mathrm{~J} 16333$ & $\begin{array}{l}\text { ICP metals, mercury, SVOA, PCBs, pesticides, } \\
\text { TPH, IC anions, total cyanide, sulfides }\end{array}$ \\
\hline SE-3 & Soil & $\begin{array}{l}\text { N } 147198.5 \\
\text { E } 581052.6\end{array}$ & $\mathrm{~J} 16335$ & $\begin{array}{l}\text { ICP metals, mercury, SVOA, PCBs, pesticides, } \\
\text { TPH, IC anions, total cyanide, sulfides }\end{array}$ \\
\hline SE-4 & Soil & $\begin{array}{l}\text { N } 147193.4 \\
\text { E } 581058.9\end{array}$ & $\mathrm{~J} 16336$ & $\begin{array}{l}\text { ICP metals, mercury, SVOA, PCBs, pesticides, } \\
\text { TPH, IC anions, total cyanide, sulfides }\end{array}$ \\
\hline SE-5 & Soil & $\begin{array}{l}\text { N } 147188.4 \\
\text { E } 581065.2\end{array}$ & $\mathrm{~J} 16337$ & $\begin{array}{l}\text { ICP metals, mercury, SVOA, PCBs, pesticides, } \\
\text { TPH, IC anions, total cyanide, sulfides }\end{array}$ \\
\hline SE-6 & Soil & $\begin{array}{l}\text { N } 147201.5 \\
\text { E } 581060.1\end{array}$ & $\mathrm{~J} 16338$ & $\begin{array}{l}\text { ICP metals, mercury, SVOA, PCBs, pesticides, } \\
\text { TPH, IC anions, total cyanide, sulfides }\end{array}$ \\
\hline
\end{tabular}


Table 1. Verification Sample Summary for the 120-F-1 Waste Site. (3 Pages)

\begin{tabular}{|c|c|c|c|c|}
\hline \multirow{2}{*}{$\begin{array}{l}\text { Sample } \\
\text { Location }\end{array}$} & \multirow{2}{*}{$\begin{array}{l}\text { Sample } \\
\text { Media }\end{array}$} & $\begin{array}{c}\text { Actual } \\
\text { Coordinates }^{b}\end{array}$ & \multirow{2}{*}{$\begin{array}{l}\text { HEIS } \\
\text { Number }\end{array}$} & \multirow{2}{*}{ Sample Analysis ${ }^{c}$} \\
\hline & & $\begin{array}{l}\text { Northing } \\
\text { Easting }\end{array}$ & & \\
\hline SE-7 & Soil & $\begin{array}{l}\text { N } 147196.4 \\
\text { E } 581066.5\end{array}$ & $\mathrm{~J} 16339$ & $\begin{array}{l}\text { ICP metals, mercury, SVOA, PCBs, pesticides, } \\
\text { TPH, IC anions, total cyanide, sulfides }\end{array}$ \\
\hline SE-8 & Soil & $\begin{array}{l}\text { N } 147191.3 \\
\text { E } 581072.8\end{array}$ & $\mathrm{~J} 16340$ & $\begin{array}{l}\text { ICP metals, mercury, SVOA, PCBs, pesticides, } \\
\text { TPH, IC anions, total cyanide, sulfides }\end{array}$ \\
\hline SE-9 & Soil & $\begin{array}{l}\text { N } 147199.4 \\
\text { E } 581074.0\end{array}$ & J16341 & $\begin{array}{l}\text { ICP metals, mercury, SVOA, PCBs, pesticides, } \\
\text { TPH, IC anions, total cyanide, sulfides }\end{array}$ \\
\hline SE-10 & Soil & $\begin{array}{l}\text { N } 147194.3 \\
\text { E } 581080.4\end{array}$ & $\mathrm{~J} 16342$ & $\begin{array}{l}\text { ICP metals, mercury, SVOA, PCBs, pesticides, } \\
\text { TPH, IC anions, total cyanide, sulfides }\end{array}$ \\
\hline SE-1 & Soil & $\begin{array}{l}\text { N } 147190.5 \\
\text { E } 581051.3\end{array}$ & $\mathrm{~J} 16 \mathrm{~B} 36$ & Hexavalent chromium \\
\hline SE-2 & Soil & $\begin{array}{l}\text { N } 147185.4 \\
\text { E } 581057.7\end{array}$ & $\mathrm{~J} 16 \mathrm{~B} 37$ & Hexavalent chromium \\
\hline SE-3 & Soil & $\begin{array}{l}\text { N } 147198.5 \\
\text { E } 581052.6\end{array}$ & $\mathrm{~J} 16 \mathrm{~B} 38$ & Hexavalent chromium \\
\hline SE-4 & Soil & $\begin{array}{l}\text { N } 147193.4 \\
\text { E } 581058.9\end{array}$ & J16B39 & Hexavalent chromium \\
\hline SE-5 & Soil & $\begin{array}{l}\text { N } 147188.4 \\
\text { E } 581065.2\end{array}$ & $\mathrm{~J} 16 \mathrm{~B} 40$ & Hexavalent chromium \\
\hline SE-6 & Soil & $\begin{array}{l}\text { N } 147201.5 \\
\text { E } 581060.1\end{array}$ & $\mathrm{~J} 16 \mathrm{~B} 41$ & Hexavalent chromium \\
\hline SE-7 & Soil & $\begin{array}{l}\text { N } 147196.4 \\
\text { E } 581066.5\end{array}$ & $\mathrm{~J} 16 \mathrm{~B} 42$ & Hexavalent chromium \\
\hline $\mathrm{SE}-8$ & Soil & $\begin{array}{l}\text { N } 147191.3 \\
\text { E } 581072.8\end{array}$ & $\mathrm{~J} 16 \mathrm{~B} 43$ & Hexavalent chromium \\
\hline SE-9 & Soil & $\begin{array}{l}\text { N } 147199.4 \\
\text { E } 581074.0\end{array}$ & $\mathrm{~J} 16 \mathrm{~B} 44$ & Hexavalent chromium \\
\hline SE-10 & Soil & $\begin{array}{l}\text { N } 147194.3 \\
\text { E } 581080.4\end{array}$ & $\mathrm{~J} 16 \mathrm{~B} 45$ & Hexavalent chromium \\
\hline$N W-1$ & Soil & $\begin{array}{l}\text { N } 147211.5 \\
\text { E } 581035.4\end{array}$ & J16DT7 & $\begin{array}{l}\text { ICP metals, mercury, hexavalent chromium, } \\
\text { pesticides, SVOA, PCBs, IC anions }\end{array}$ \\
\hline NW-2 & Soil & $\begin{array}{l}\text { N } 147210.0 \\
\text { E } 581041.3\end{array}$ & J16DT8 & $\begin{array}{l}\text { ICP metals, mercury, hexavalent chromium, } \\
\text { pesticides, SVOA, PCBs, IC anions }\end{array}$ \\
\hline NW-3 & Soil & $\begin{array}{l}\text { N } 147217.4 \\
\text { E } 581033.8\end{array}$ & J16DT9 & $\begin{array}{l}\text { ICP metals, mercury, hexavalent chromium, } \\
\text { pesticides, SVOA, PCBs, IC anions }\end{array}$ \\
\hline NW-4 & Soil & $\begin{array}{l}\text { N } 147215.9 \\
\text { E } 581039.7\end{array}$ & J16DV0 & $\begin{array}{l}\text { ICP metals, mercury, hexavalent chromium, } \\
\text { pesticides, SVOA, PCBs, IC anions }\end{array}$ \\
\hline NW-5 & Soil & $\begin{array}{l}\text { N } 147214.3 \\
\text { E } 581045.6\end{array}$ & J16DV 1 & $\begin{array}{l}\text { ICP metals, mercury, hexavalent chromium, } \\
\text { pesticides, SVOA, PCBs, IC anions }\end{array}$ \\
\hline
\end{tabular}


Table 1. Verification Sample Summary for the 120-F-1 Waste Site. (3 Pages)

\begin{tabular}{|c|c|c|c|c|}
\hline \multirow{2}{*}{$\begin{array}{l}\text { Sample } \\
\text { Location }\end{array}$} & \multirow{2}{*}{$\begin{array}{l}\text { Sample } \\
\text { Media }\end{array}$} & $\begin{array}{c}\text { Actual } \\
\text { Coordinates }^{b}\end{array}$ & \multirow{2}{*}{$\begin{array}{l}\text { HEIS } \\
\text { Number }\end{array}$} & \multirow{2}{*}{ Sample Analysis ${ }^{c}$} \\
\hline & & $\begin{array}{l}\text { Northing } \\
\text { Easting }\end{array}$ & & \\
\hline NW-6 & Soil & $\begin{array}{l}\text { N } 147212.7 \\
\text { E } 581051.5\end{array}$ & J16DV2 & $\begin{array}{l}\text { ICP metals, mercury, hexavalent chromium, } \\
\text { pesticides, SVOA, PCBs, IC anions }\end{array}$ \\
\hline NW-7 & Soil & $\begin{array}{l}\text { N } 147221.8 \\
\text { E } 581038.1\end{array}$ & J16DV3 & $\begin{array}{l}\text { ICP metals, mercury, hexavalent chromium, } \\
\text { pesticides, SVOA, PCBs, IC anions }\end{array}$ \\
\hline$N W-8$ & Soil & $\begin{array}{l}\text { N } 147220.2 \\
\text { E } 581044.0\end{array}$ & J16DV4 & $\begin{array}{l}\text { ICP metals, mercury, hexavalent chromium, } \\
\text { pesticides, SVOA, PCBs, IC anions }\end{array}$ \\
\hline NW-9 & Soil & $\begin{array}{l}\text { N } 147218.6 \\
\text { E } 581049.9\end{array}$ & J16DV5 & $\begin{array}{l}\text { ICP metals, mercury, hexavalent chromium, } \\
\text { pesticides, SVOA, PCBs, IC anions }\end{array}$ \\
\hline NW-10 & Soil & $\begin{array}{l}\text { N } 147217.1 \\
\text { E } 581055.8\end{array}$ & J16DV6 & $\begin{array}{l}\text { ICP metals, mercury, hexavalent chromium, } \\
\text { pesticides, SVOA, PCBs, IC anions }\end{array}$ \\
\hline $\begin{array}{l}\text { Duplicate of } \\
\text { J16333 }\end{array}$ & Soil & $\begin{array}{l}\text { N } 147185.4 \\
\text { E } 581057.7\end{array}$ & $\mathrm{~J} 16334$ & $\begin{array}{l}\text { ICP metals, mercury, SVOA, PCBs, pesticides, } \\
\text { TPH, IC anions, total cyanide, sulfides }\end{array}$ \\
\hline $\begin{array}{l}\text { Duplicate of } \\
\text { J16B46 }\end{array}$ & Soil & $\begin{array}{l}\text { N } 147194.3 \\
\text { E } 581080.4\end{array}$ & $\mathrm{~J} 16 \mathrm{~B} 46$ & Hexavalent chromium \\
\hline $\begin{array}{l}\text { Duplicate of } \\
\text { J16DV7 }\end{array}$ & Soil & $\begin{array}{l}\text { N } 147217.1 \\
\text { E } 581055.8\end{array}$ & J16DV6 & $\begin{array}{l}\text { ICP metals, mercury, hexavalent chromium, } \\
\text { pesticides, SVOA, PCBs, IC anions }\end{array}$ \\
\hline $\begin{array}{l}\text { Equipment } \\
\text { Blank }\end{array}$ & Silica sand & NA & J16354 & ICP metals, IC anions \\
\hline $\begin{array}{l}\text { Equipment } \\
\text { Blank }\end{array}$ & Silica sand & NA & J16DT6 & ICP metals, IC anions \\
\hline
\end{tabular}

a Source: Field logbook EFL-1174-4, pp. 27-29 (WCH 2007b) and 88-89 (WCH 2008b).

b Washington State Plane (meters).

c Analyses of IC anions, total cyanide, sulfides were inadvertently requested for the southeast excavation samples instead of hexavalent chromium. These analyses were not required for the verification sampling. Due to detections of some anions, this analysis was added to the northwest excavation samples for consistency. Hexavalent chromium samples for the southeast excavation were collected at a later time.

HEIS = Hanford Environmental Information System

ICP = inductively coupled plasma

IC = ion chromatography

$\mathrm{NA}=$ not applicable

$\mathrm{PCB}=$ polychlorinated biphenyl

SVOA $=$ semi-volatile organic analysis

$\mathrm{TPH}=$ total petroleum hydrocarbons 
Figure 3. 120-F-1 Verification Sample Locations.

G: \RS_SamplingFigures \100F \120-F-1_Fig2.dwg
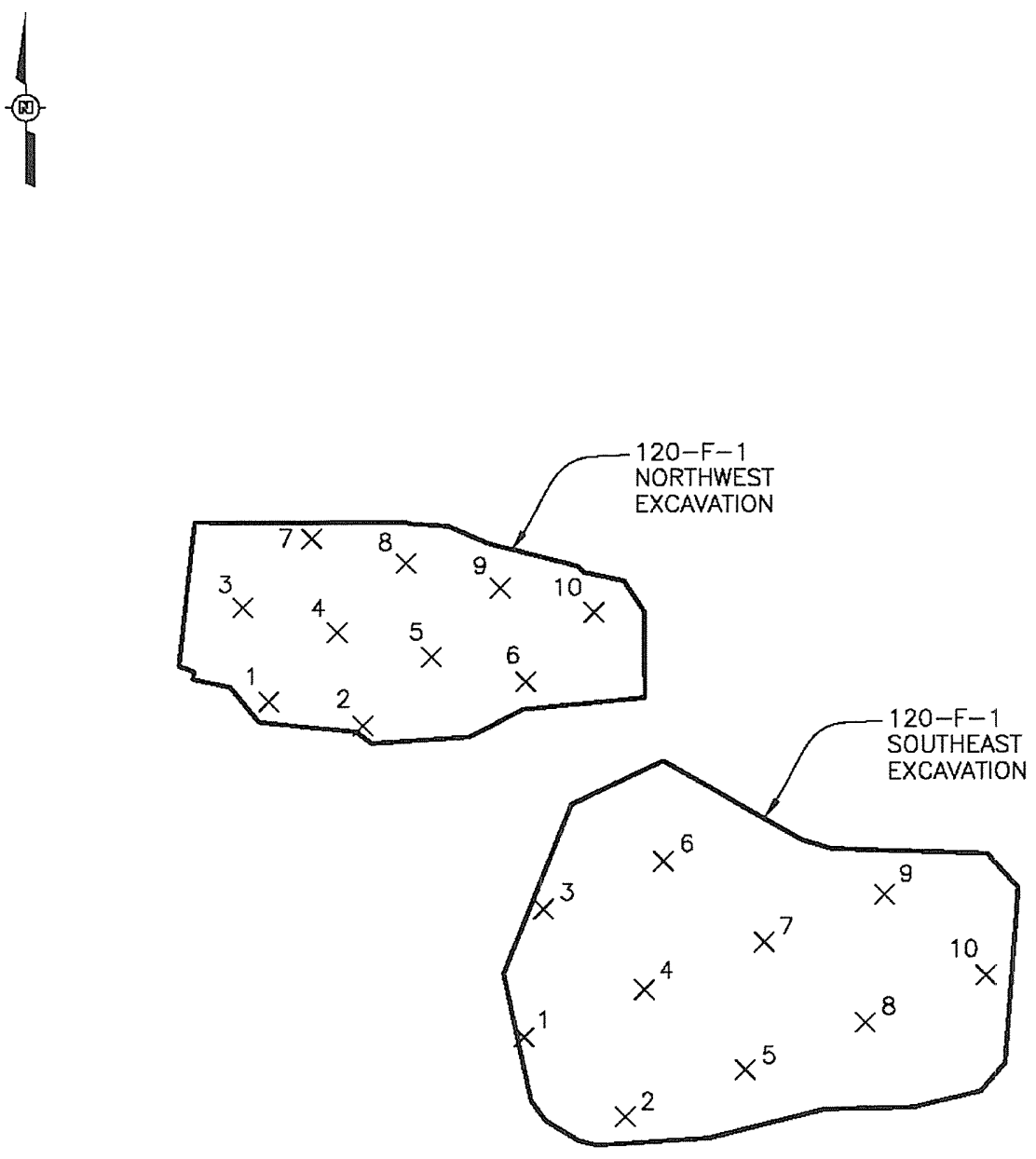

\section{Legend}

$x^{1} \quad$ Verification Sampling Points

SCALE 1:500

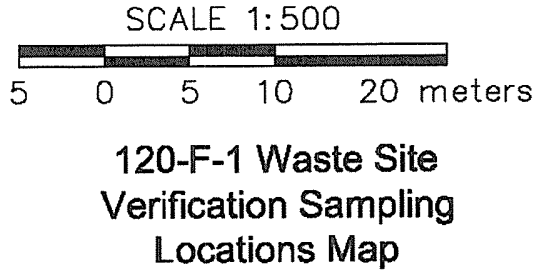




\section{Verification Sampling Results}

Verification samples were analyzed using U.S. Environmental Protection Agency-approved analytical methods. The laboratory-reported data results for all constituents are stored in the Environmental Restoration project-specific database prior to archival in the Hanford Environmental Information System and are presented in Attachment 1 of the 95\% UCL calculation in Appendix C.

As noted earlier, the 120-F-1 waste site was divided into two sampling areas for verification sampling: the northwest excavation and the southeast excavation. Evaluation of the verification data from the excavation footprint was calculated using the 95\% UCL on the true population mean for residual concentrations of COPCs as specified by the RDR/RAWP (DOE-RL 2005b). These calculations are provided in Appendix C. When a COPC was detected in fewer than 50\% of the verification samples collected, the maximum detected value was used for comparison against the RAGs. If no detections for a given COPC were reported in the data set, then no statistical evaluation or calculations were performed for that COPC.

Comparisons of the statistical and maximum results for COPCs with the shallow zone RAGs for the southeast and northwest excavation footprints are summarized in Tables $2 \mathrm{a}$ and $2 \mathrm{~b}$, respectively. Both sampling areas are evaluated using the more restrictive shallow zone cleanup criteria, even though a portion of the southeast excavation exceeded $4.6 \mathrm{~m}(15 \mathrm{ft})$ in depth. Contaminants that were not detected by laboratory analysis are excluded from these tables. Calculated cleanup levels are not presented in the Cleanup Levels and Risk Calculations Database (Ecology 2005) under Washington Administrative Code (WAC) 173-340-740(3) for aluminum, calcium, iron, magnesium, potassium, silicon, and sodium; therefore, these constituents are not considered site COPCs.

Table 2a. Comparison of Maximum or Statistical Contaminant Concentrations to Action Levels for the 120-F-1 Southeast Verification Sampling Event. (2 Pages)

\begin{tabular}{|c|c|c|c|c|c|c|}
\hline \multirow[b]{2}{*}{ COPC } & \multirow{2}{*}{$\begin{array}{c}\text { Statistical or } \\
\text { Maximum Result } \\
(\mathbf{m g} / \mathrm{kg})\end{array}$} & \multicolumn{3}{|c|}{ Soil Cleanup Levels, $(\mathrm{mg} / \mathrm{kg})^{\mathrm{a}}$} & \multirow{2}{*}{$\begin{array}{l}\text { Does the } \\
\text { Maximum } \\
\text { Exceed } \\
\text { RAGs? }\end{array}$} & \multirow{2}{*}{$\begin{array}{l}\text { Does the } \\
\text { Result Pass } \\
\text { RESRAD } \\
\text { Modeling? }\end{array}$} \\
\hline & & $\begin{array}{c}\text { Direct } \\
\text { Exposure }\end{array}$ & $\begin{array}{l}\text { Protective of } \\
\text { Groundwater }\end{array}$ & $\begin{array}{c}\text { Protective } \\
\text { of the River }\end{array}$ & & \\
\hline Arsenic & $2.9(<\mathrm{BG})$ & 20 & 20 & 20 & No & -- \\
\hline Barium & $58.2(<\mathrm{BG})$ & 5,600 & $132^{b}$ & 224 & No & - \\
\hline Beryllium & $0.73(<\mathrm{BG})$ & $10.4^{\mathrm{c}}$ & $1.51^{\mathrm{b}}$ & $1.51^{\mathrm{b}}$ & No & -- \\
\hline Boron $^{d}$ & 4.6 & 16,000 & 320 & $\mathrm{NA}$ & No & - \\
\hline Chromium, total & $7.4(<\mathrm{BG})$ & 80,000 & $18.5^{\mathrm{b}}$ & $18.5^{\mathrm{b}}$ & No & -- \\
\hline Cobalt & $5.3(<\mathrm{BG})$ & 1,600 & 32 & $\mathrm{NA}$ & No & - \\
\hline Copper & $12.6(<\mathrm{BG})$ & 2,960 & 59.2 & $22.0^{6}$ & No & -- \\
\hline Hexavalent chromium $^{\mathrm{d}}$ & 1.8 & $2.1^{\mathrm{c}}$ & 4.8 & 2 & No & -- \\
\hline Lead & $6.1(<\mathrm{BG})$ & 353 & $10.2^{b}$ & $10.2^{b}$ & No & -- \\
\hline Manganese & $259(<\mathrm{BG})$ & 11,200 & $512^{b}$ & $512^{b}$ & No & - \\
\hline Mercury & 0.65 & 24 & $0.33^{b}$ & $0.33^{b}$ & Yes & Yes $^{e}$ \\
\hline Molybdenum $^{\mathrm{d}}$ & 0.85 & 400 & 8 & NA & No & -- \\
\hline Nickel & $9.3(<\mathrm{BG})$ & 1,600 & $19.1^{b}$ & 27.4 & No & -- \\
\hline
\end{tabular}


Table 2a. Comparison of Maximum or Statisticall Contaminant Concentrations to Action Levels for the 120-F-1 Southeast Verification Sampling Event. (2 Pages)

\begin{tabular}{|c|c|c|c|c|c|c|}
\hline \multirow[b]{2}{*}{ COPC } & \multirow{2}{*}{$\begin{array}{c}\text { Statistical or } \\
\text { Maximum Result } \\
(\mathrm{mg} / \mathrm{kg})\end{array}$} & \multicolumn{3}{|c|}{ Soil Cleanup Levels, $(\mathrm{mg} / \mathrm{kg})^{\mathrm{a}}$} & \multirow{2}{*}{$\begin{array}{l}\text { Does the } \\
\text { Maximum } \\
\text { Exceed } \\
\text { RAGs? }\end{array}$} & \multirow{2}{*}{$\begin{array}{l}\text { Does the } \\
\text { Result Pass } \\
\text { RESRAD } \\
\text { Modeling? }\end{array}$} \\
\hline & & $\begin{array}{c}\text { Direct } \\
\text { Exposure }\end{array}$ & $\begin{array}{l}\text { Protective of } \\
\text { Groundwater }\end{array}$ & $\begin{array}{c}\text { Protective } \\
\text { of the River }\end{array}$ & & \\
\hline Vanadium & $38.5(<\mathrm{BG})$ & 560 & $85.1^{\mathrm{b}}$ & NA & No & -- \\
\hline Zinc & $37.5(<\mathrm{BG})$ & 24,000 & 480 & $67.8^{b}$ & No & - \\
\hline Fluoride & 3.9 & 4,800 & 96 & NA & No & - \\
\hline Nitrate (as Nitrogen) & $4.2(<\mathrm{BG})$ & 128,000 & 1,000 & 2,000 & $\mathrm{No}$ & -- \\
\hline Sulfate & $8,410^{f}$ & NA & 25,000 & 50,000 & $\mathrm{No}$ & -- \\
\hline $\begin{array}{l}\text { Bis(2-ethylhexyl) } \\
\text { phthalate }\end{array}$ & 0.11 & 71.4 & 0.625 & 0.36 & No & - \\
\hline Aroclor- 1254 & 0.023 & 0.5 & $0.017^{\mathrm{g}}$ & $0.017^{\mathrm{g}}$ & No & $\cdots$ \\
\hline Aroclor- 1260 & 0.010 & 0.5 & $0.017^{\mathrm{g}}$ & $0.017^{\mathrm{g}}$ & No & - \\
\hline Dibenz $(\mathrm{a}, \mathrm{h})$ anthracene & 0.025 & $0.33^{\mathrm{g}}$ & $0.33^{\mathrm{g}}$ & $0.33^{\mathrm{g}}$ & No & - \\
\hline alpha-Chlordane & 0.010 & 0.769 & $0.0165^{\mathrm{g}}$ & $0.0165^{\mathrm{g}}$ & No & -- \\
\hline gamma-Chlordane & 0.013 & 0.769 & $0.0165^{\mathrm{g}}$ & $0.0165^{\mathrm{g}}$ & No & -- \\
\hline DDE & 0.0018 & 2.94 & 0.0257 & $0.0033^{\mathrm{g}}$ & No & -- \\
\hline DDT & 0.0021 & 2.94 & 0.0257 & $0.0033^{g}$ & No & -- \\
\hline Endosulfan I & 0.0018 & 480 & 9.6 & 0.0112 & No & -- \\
\hline
\end{tabular}

" Lookup values and RAGs obtained from the Remedial Design Report/Remedial Action Work Plan for the 100 Area (DOE-RL 2005b) or calculated per WAC 173-340-720, WAC 173-340-730, and WAC 173-340-740, Method B, 1996, unless otherwise noted.

b Where cleanup levels are less than background, cleanup levels default to background per WAC 173-340-700[4][d] (Ecology 1996).

${ }^{c}$ Carcinogenic cleanup level calculated based on the inhalation exposure pathway (WAC 173-340-750[3]) (Ecology 1996) using an airborne particulate mass-loading rate of $0.0001 \mathrm{~g} / \mathrm{m}^{3}$ (WDOH 1997).

d No Hanford Site-specific or Washington State background value available.

e Based on the 100 Area Analogous Sites RESRAD Calculations (BHI 2005), residual concentrations of mercury are not expected to migrate more than $2 \mathrm{~m}(6.6 \mathrm{ft})$ vertically in 1,000 years based on the soil-partitioning distribution coefficient for mercury of $30 \mathrm{~mL} / \mathrm{g}$. The vadose zone underlying the remediation footprint is approximately $6.6 \mathrm{~m}(21.7 \mathrm{ft})$ thick. Therefore, residual concentrations of mercury are predicted to be protective of groundwater and the Columbia River.

$f$ See sulfate data discussion in following section.

\& Where cleanup levels are less than RDLs, cleanup levels default to RDLs per WAC 173-340-707(2) (Ecology 1996).

- $\quad=$ not applicable

$\mathrm{BG} \quad=$ background

RAG = remedial action goal

RDL $\quad=$ required detection limit

$\mathrm{COPC}=$ contaminant of potential concern

$\mathrm{NA}=$ not available

RESRAD = RESidual RADioactivity (dose assessment model)

WAC $=$ Washington Administrative Code

Table $2 \mathrm{~b}$. Comparison of Maximum or Statistical Contaminant Concentrations to Action Levels for the 120-F-1 Northwest Verification Sampling Event. (2 Pages)

\begin{tabular}{|c|c|c|c|c|c|c|}
\hline \multirow[b]{2}{*}{ COPC } & \multirow[b]{2}{*}{$\begin{array}{c}\text { Statistical or } \\
\text { Maximum Result } \\
(\mathrm{mg} / \mathrm{kg})\end{array}$} & \multicolumn{3}{|c|}{ Soil Cleanup Levels, $(\mathrm{mg} / \mathrm{kg})^{\mathrm{a}}$} & \multirow{2}{*}{$\begin{array}{l}\text { Does the } \\
\text { Maximum } \\
\text { Exceed } \\
\text { RAGs? }\end{array}$} & \multirow{2}{*}{$\begin{array}{l}\text { Does the } \\
\text { Result Pass } \\
\text { RESRAD } \\
\text { Modeling? }\end{array}$} \\
\hline & & $\begin{array}{c}\text { Direct } \\
\text { Exposure }\end{array}$ & $\begin{array}{l}\text { Protective of } \\
\text { Groundwater }\end{array}$ & $\begin{array}{l}\text { Protective } \\
\text { of the River }\end{array}$ & & \\
\hline Antimony & $0.91(<\mathrm{BG})$ & 32 & $5^{b}$ & $5^{b}$ & $\mathrm{No}$ & \\
\hline Arsenic & $2.5(<\mathrm{BG})$ & 20 & 20 & 20 & No & -- \\
\hline Barium & $65.8(<\mathrm{BG})$ & 5,600 & $132^{\mathrm{b}}$ & 224 & No & $\cdots$ \\
\hline Beryllium & $0.26(<\mathrm{BG})$ & $10.4^{\mathrm{c}}$ & $1.51^{\mathrm{b}}$ & $1.51^{b}$ & No & - \\
\hline
\end{tabular}




\section{Table 2b. Comparison of Maximum or Statistical Contaminant Concentrations to Action} Levels for the 120-F-1 Northwest Verification Sampling Event. (2 Pages)

\begin{tabular}{|c|c|c|c|c|c|c|}
\hline \multirow[b]{2}{*}{$\mathrm{COPC}$} & \multirow{2}{*}{$\begin{array}{c}\text { Statistical or } \\
\text { Maximum Result } \\
(\mathrm{mg} / \mathrm{kg})\end{array}$} & \multicolumn{3}{|c|}{ Soil Cleanup Levels, $(\mathrm{mg} / \mathrm{kg})^{\mathrm{a}}$} & \multirow{2}{*}{$\begin{array}{l}\text { Does the } \\
\text { Maximum } \\
\text { Exceed } \\
\text { RAGs? }\end{array}$} & \multirow{2}{*}{$\begin{array}{c}\text { Does the } \\
\text { Result Pass } \\
\text { RESRAD } \\
\text { Modeling? }\end{array}$} \\
\hline & & $\begin{array}{c}\text { Direct } \\
\text { Exposure }\end{array}$ & $\begin{array}{l}\text { Protective of } \\
\text { Groundwater }\end{array}$ & $\begin{array}{l}\text { Protective } \\
\text { of the River }\end{array}$ & & \\
\hline Boron $^{d}$ & 1.6 & 16,000 & 320 & NA & No & -- \\
\hline Chromium, total & $11.7(<\mathrm{BG})$ & 80,000 & $18.5^{b}$ & $18.5^{b}$ & No & -- \\
\hline Cobalt & $7.1(<\mathrm{BG})$ & 1,600 & 32 & NA & No & $-\ldots$ \\
\hline Copper & $12.2(<\mathrm{BG})$ & 2,960 & 59.2 & $22.0^{b}$ & No & -- \\
\hline Hexavalent chromium $^{d}$ & 0.30 & $2.1^{\mathrm{c}}$ & 4.8 & 2 & No & -- \\
\hline Lead & $2.9(<\mathrm{BG})$ & 353 & $10.2^{b}$ & $10.2^{b}$ & No & -- \\
\hline Manganese & $318(<\mathrm{BG})$ & 11,200 & $512^{b}$ & $512^{b}$ & No & - \\
\hline Nickel & $11.8(<\mathrm{BG})$ & 1,600 & $19.1^{b}$ & 27.4 & No & -- \\
\hline Selenium & 1.8 & 400 & 5 & 1 & Yes & Yes $^{\mathrm{e}}$ \\
\hline Vanadium & $53.9(<\mathrm{BG})$ & 560 & 85.1 & $\mathrm{NA}$ & No & -- \\
\hline Zinc & $37.3(<\mathrm{BG})$ & 24,000 & 480 & $67.8^{b}$ & No & -- \\
\hline Nitrate (as Nitrogen) & $5.7(<\mathrm{BG})$ & 128,000 & 1,000 & 2,000 & No & -- \\
\hline Sulfate & $6.4(<\mathrm{BG})$ & NA & 25,000 & 50,000 & No & -- \\
\hline $\begin{array}{l}\text { Bis(2-ethylhexyl) } \\
\text { phthalate }\end{array}$ & 0.12 & 71.4 & 0.625 & 0.36 & No & -- \\
\hline Di-n-butylphthalate & 0.027 & 8,000 & 160 & 540 & No & -- \\
\hline alpha-Chlordane & 0.0021 & 0.769 & $0.02^{f}$ & $0.02^{\mathrm{f}}$ & No & $\cdots$ \\
\hline gamma-Chlordane & 0.0022 & 0.769 & $0.02^{f}$ & $0.02^{f}$ & No & -- \\
\hline
\end{tabular}

" Lookup values and RAGs obtained from the Remedial Design Report/Remedial Action Work Plan for the 100 Area (DOE-RL 2005b) or calculated per WAC 173-340-720, WAC 173-340-730, and WAC 173-340-740, Method B, 1996, unless otherwise noted.

b Where cleanup levels are less than background, cleanup levels default to background per WAC 173-340-700[4][d] (Ecology 1996).

c Carcinogenic cleanup level calculated based on the inhalation exposure pathway (WAC 173-340-750[3]) (Ecology 1996) using an airborne particulate mass-loading rate of $0.0001 \mathrm{~g} / \mathrm{m}^{3}$ (WDOH 1997).

d No Hanford Site-specific or Washington State background value available.

e Based on the 100 Area Analogous Sites RESRAD Calculations (BHI 2005), residual concentrations of selenium are not expected to

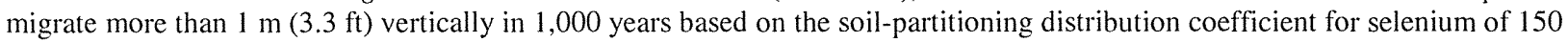
$\mathrm{mL} / \mathrm{g}$. The vadose zone underlying the remediation footprint is approximately $6.6 \mathrm{~m}(21.7 \mathrm{ft})$ thick. Therefore, residual concentrations of selenium are predicted to be protective of groundwater and the Columbia River.

$f$ Where cleanup levels are less than RDLs, cleanup levels default to RDLs per WAC 173-340-707(2) (Ecology 1996).

$-\quad=$ not applicable $\quad$ RAG $\quad=$ remedial action goal

$\mathrm{BG}=$ background $\quad \mathrm{RDL} \quad$ = required detection limit

COPC = contaminant of potential concern $\quad$ RESRAD $=$ RESidual RADioactivity (dose assessment model)

$\mathrm{NA}=$ not available $\quad \mathrm{WAC}=$ Washington Administrative Code

\section{VERIFICATION SAMPLE DATA EVALUATION}

Evaluation of the verification sampling results in Tables $2 \mathrm{a}$ and $2 \mathrm{~b}$ show that all direct exposure cleanup levels are met for the two sampling areas within the 120-F-1 waste site.

In the southeast excavation (Table 2a), groundwater and Columbia River protection RAGs were exceeded for mercury based on a single sample result. Analysis of the remaining nine samples did not detect mercury. Data were not collected on the vertical extent of residual contamination, but RESidual RADioactivity (RESRAD) modeling predicts that compounds having a soil- 
partitioning coefficient $\left(\mathrm{K}_{\mathrm{d}}\right)$ greater than $12 \mathrm{~mL} / \mathrm{g}$ will not migrate through the $6.6 \mathrm{~m}$ (21.7-ft-) thick vadose zone between the shallow zone and groundwater at the deeper southeast portion of the waste site (BHI 2005). The $\mathrm{K}_{\mathrm{d}}$ for mercury is $50 \mathrm{~mL} / \mathrm{g}$ and, as discussed above, is not expected to migrate through the vadose zone. Therefore, the remediation performed in the southeast excavation of the $120-\mathrm{F}-1$ waste site is protective of groundwater and the Columbia River.

In the northwest excavation (Table $2 b$ ), the Columbia River protection RAG was exceeded for selenium based on a single sample result. Analyses of the remaining nine samples did not detect selenium. Data were not collected on the vertical extent of residual contamination, but RESRAD modeling predicts that compounds having a soil-partitioning coefficient $\left(\mathrm{K}_{\mathrm{d}}\right)$ greater than $8 \mathrm{~mL} / \mathrm{g}$ will not migrate through the $10.5 \mathrm{~m}$ (34.4-ft-) thick vadose zone between the shallow zone and groundwater at the shallower, northwest excavation of the site (BHI 2005). The $\mathrm{K}_{\mathrm{d}}$ for selenium is $150 \mathrm{~mL} / \mathrm{g}$ and, as discussed above, selenium is not predicted to migrate through the vadose zone within 1,000 years. Therefore, the remediation performed in the northwest excavation of the $120-\mathrm{F}-1$ waste site is protective of groundwater and the Columbia River.

All other COPCs for the 120-F-1 waste site were either not detected or were quantified below RAGs.

When using a statistical sampling approach, a RAG requirement for nonradionuclides is the WAC 173-340-740(7)(e) three-part test. The application of the three-part test for the 120-F-1 waste site is included in the statistical calculations (Appendix C). All residual COPC concentrations for both excavations within the 120-F-1 waste site pass the three-part test.

Assessment of the risk requirements for the 120-F-1 waste site is determined by calculation of the hazard quotient and carcinogenic (excess cancer) risk values for nonradionuclides. These calculations are located in Appendix $\mathrm{C}$. The requirements include an individual hazard quotient of less than 1.0, a cumulative hazard quotient of less than 1.0, an individual contaminant carcinogenic risk of less than $1 \times 10^{-6}$, and a cumulative excess carcinogenic risk of less than $1 \times 10^{-5}$. These risk values were conservatively calculated for the entire waste site using the highest values from each of the sampling areas. Risk values were not calculated for constituents that were not detected or were detected at concentrations below Hanford Site or Washington State background values. The calculations indicated that all individual hazard quotients for noncarcinogenic constituents are less than 1.0. The cumulative hazard quotient for the 120-F-1 waste site is $4.2 \times 10^{-2}$. All individual cumulative carcinogenic risk values are less than $1 \times 10^{-6}$. The cumulative carcinogenic risk value is $1.1 \times 10^{-6}$. Therefore, nonradionuclide risk requirements are met.

\section{$\underline{\text { Sulfate Data Discussion }}$}

The sulfate data analysis in the southeast excavation was problematic due to a spread of three orders of magnitude in the sample results and the use of a lognormal distribution to calculate the $95 \%$ UCL. A $95 \%$ UCL value of $1,740,000 \mathrm{mg} / \mathrm{kg}$ was obtained from the 10 sample results (censored) using Ecology MTCAStat software and a lognormal distribution (Figure 4).

MTCAStat software uses Land's method of statistical calculation and a H-statistic when the data 
is determined to be lognormal. However, the maximum sulfate result from these 10 samples was $8,410 \mathrm{mg} / \mathrm{kg}$ with a mean of $5,202 \mathrm{mg} / \mathrm{kg}$ and a standard deviation of $2,660 \mathrm{mg} / \mathrm{kg}$ (Figure 4).

The 95\% UCL result from MTCAStat software using a lognormal distribution and a H-statistic does not provide a realistic upper value for sulfate. Significant sample heterogeneity is apparent in the primary and duplicate samples for these samples where a 92\% relative percent difference was calculated for sulfate (Appendix C). While $80 \%$ of the sulfate data were above the detection limit indicating a lognormal MTCAStat analysis is suitable, the maximum sulfate result is the appropriate value to use for comparison against the RAGs in this case.

The sulfate data were analyzed using ProUCL version 4.0 (EPA 2007) to determine if a better statistical test was appropriate. Results from this analysis indicated the data was gamma distributed and suggested using an adjusted gamma UCL (Figure 5). The 95\% UCL value for sulfate using this test was $7,396 \mathrm{mg} / \mathrm{kg}$.

The groundwater protection RAG for sulfate is based on a secondary maximum contaminant level (MCL) value. This RAG $(25,000 \mathrm{mg} / \mathrm{kg})$ is nearly three times the maximum value from the sample set $(8,410 \mathrm{mg} / \mathrm{kg})$. There is no direct exposure value for sulfate. There is a health based, drinking water advisory level for sulfate of $500 \mathrm{mg} / \mathrm{L}$ from EPA (EPA 2003). This equates to a soil concentration value of $50,000 \mathrm{mg} / \mathrm{kg}$ using the $100 \mathrm{X}$ rule as prescribed in the RDR/RAWP (DOE-RL 2005b).

For the sulfate results in the 120-F-1 southeast excavation, the MTCAStat 95\% UCL value does not provide a realistic upper bound of concentration. Furthermore, the source of the sulfate RAG is a secondary MCL, which is driven by aesthetic concerns, not health risks. Therefore, it is appropriate to use the maximum sample result for comparison against the groundwater and Columbia River protection RAGs.

\section{VERIFICATION SAMPLING DATA QUALITY ASSESSMENT}

A data quality assessment (DQA) is performed to compare the verification sampling approach, the field logbook (WCH 2008b), and resulting analytical data with the sampling and data quality requirements specified by the project objectives and performance specifications. 
Figure 4. MTCAStat calculation for sulfate results from 120-F-1 southeast excavation.

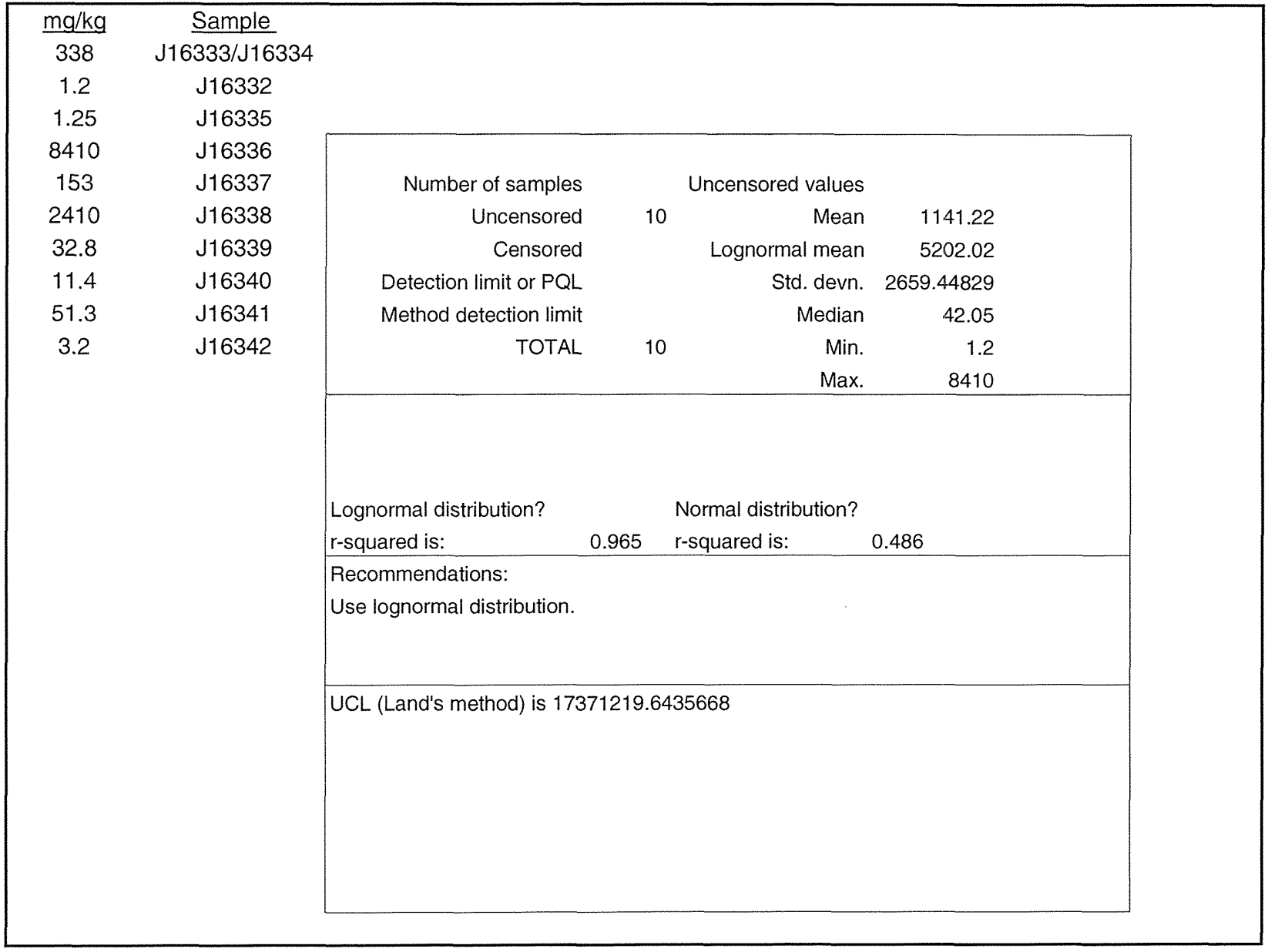




\section{Figure 5. Results of 95\% UCL Analysis of Sulfate Results for Southeast Excavation Using ProUCL 4.0.}

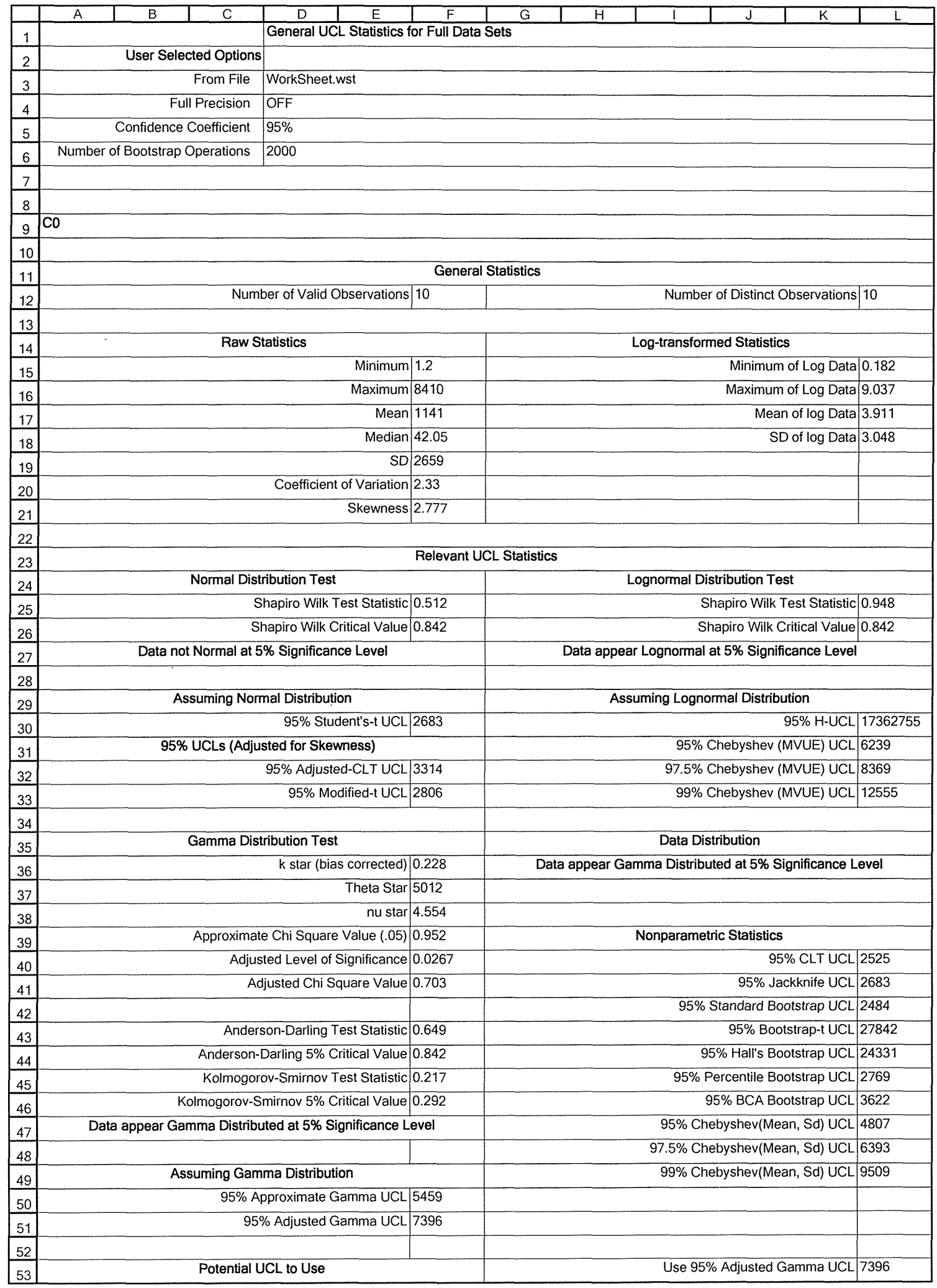


The DQA for the 120-F-1 waste site established that the data are of the right type, quality, and quantity to support site verification decisions within specified error tolerances. All analytical data were found to be acceptable for decision-making purposes. The evaluation verified that the sample design was sufficient for the purpose of clean site verification. The cleanup verification sample analytical data are stored in the ENRE project-specific database for data evaluation prior to its archival in the HEIS and are summarized in Appendix C. The detailed DQA is presented in Appendix E.

\section{SUMMARY FOR INTERIM CLOSURE}

The 120-F-1 glass dump waste site has been remediated in accordance with the Remaining Sites ROD (EPA 1999) and the RDR/RAWP (DOE-RL 2005b). The site was remediated by removing approximately 1,505 BCM of material for disposal at the ERDF. Statistical sampling to verify the completeness of remediation was performed, and analytical results for the two decision units were shown to meet the cleanup objectives for direct exposure, groundwater protection, and river protection. Accordingly, an interim closure reclassification is supported for the 120-F-1 waste site. The 120-F-1 waste site excavation area has a maximum depth of approximately $6.5 \mathrm{~m}$ $(21 \mathrm{ft})$, which includes a shallow zone and a deep zone. However, the entire excavation area is considered one decision unit, and will be closed out using the more restrictive shallow zone cleanup criteria; therefore, institutional controls to prevent uncontrolled drilling or excavation into the deep zone are not required.

\section{REFERENCES}

40 CFR 141, "National Primary Drinking Water Regulations," Code of Federal Regulations, as amended.

BHI, 1994, 100-F Reactor Site Technical Baseline Report Including Operable Units 100-FR-1 and 100-FR-2, BHI-00031, Rev. 0, Bechtel Hanford, Inc., Richland, Washington.

BHI, 1998a, 120-F-1 Glass Dump Interim Stabilization Final Report, BHI-01197, Rev. 0, Bechtel Hanford, Inc., Richland, Washington.

BHI, 1998b, Sampling and Analysis Instruction for the 120-F-1 Glass Dump Site, BHI-01022, Rev. 0, Bechtel Hanford, Inc., Richland, Washington.

BHI, 2004, Results of Geophysical Investigation at 100-F-Area Remaining Sites, Interoffice Memorandum to R. A. Carlson, CCN 112477, dated May 27, 2004, Bechtel Hanford, Inc., Richland, Washington.

BHI, 2005, 100 Area Analogous Sites RESRAD Calculations, 0100X-CA-V0050, Rev. 0, Bechtel Hanford, Inc., Richland, Washington. 
BHI, 2006, Industrial Hygiene Investigation of Potential Beryllium-Contaminated Fluorescent Light Bulbs, CCN 0571201 dated February 9, 2006, Bechtel Hanford, Inc., Richland, Washington.

DOE Order 5400.5, Radiation Protection of the Public and the Environment, as amended, U.S. Department of Energy, Washington, D.C.

DOE-RL, 2005a, 100 Area Remedial Action Sampling and Analysis Plan, DOE/RL-96-22, Rev. 4, Draft B, U.S. Department of Energy, Richland Operations Office, Richland, Washington.

DOE-RL, 2005b, Remedial Design Report/Remedial Action Work Plan for the 100 Area, DOE/RL-96-17, Rev. 5, Draft B, U.S. Department of Energy, Richland Operations Office, Richland, Washington.

DOE-RL, 2007, Tri-Party Agreement Handbook Management Procedures, RL-TPA-90-0001, Guideline Number TPA-MP-14, "Maintenance of the Waste Information Data System (WIDS)," Rev. 1, U.S. Department of Energy, Richland Operations Office, Richland, Washington.

Ecology, 1995, Washington State Department of Ecology Toxics Cleanup Program - Guidance on Sampling and Data Analysis Methods, Publication No. 94-49, Washington State Department of Ecology, Olympia, Washington.

Ecology, 1996, "Model Toxics Control Act--Cleanup," Washington Administrative Code (WAC) 173-340, Washington State Department of Ecology, Olympia, Washington

Ecology, 2005, Cleanup Levels and Risk Calculations (CLARC) Database, Washington State Department of Ecology, Olympia, Washington, $<$ https://fortress.wa.gov/ecy/clarc/CLARCHome.aspx>.

EPA, 1999, Interim Action Record of Decision for the 100-BC-1, 100-BC-2, 100-DR-1, 100-DR-2, 100-FR-1, 100-FR-2, 100-HR-1, 100-HR-2, 100-KR-1, 100-KR-2, 100-IU-2, 100-IU-6, and 200-CW-3 Operable Units, Hanford Site, Benton County, Washington, U.S. Environmental Protection Agency, Region 10, Seattle, Washington.

EPA, 2000, Guidance for Data Quality Assessment, EPA QA/G-9, QA00 Update, U.S. Environmental Protection Agency, Office of Environmental Information, Washington, D.C.

EPA, 2003, Drinking Water Advisory: Consumer Acceptability Advice and Health Effects Analysis on Sulfate, EPA 822-F-03-007, U.S. Environmental Protection Agency, Office of Water (4304T), Health and Ecological Criteria Division, Washington, D. C.

EPA, 2006, Integrated Risk Information System (IRIS), U.S. Environmental Protection Agency, Washington, D.C., available at <http://www.epa.gov/iris>. 
EPA, 2007, ProUCL, Version 4.0, U.S. Environmental Protection Agency, Washington, D.C. $<$ http://www.epa.gov/esd/tsc/install.htm>.

ENV-1, Environmental Monitoring \& Management, Washington Closure Hanford, Richland, Washington.

Gilbert, R. O., 1987, Statistical Methods for Environmental Pollution Monitoring, Wiley \& Sons, Inc., New York, New York.

PNNL, 2002, Visual Sample Plan, Version 2.0, available at http://dqo.pnl.gov/VSP, Pacific Northwest National Laboratory, Richland, Washington.

WAC 173-340, "Model Toxics Control Act -- Cleanup," Washington Administrative Code, amended 1996.

WCH, 2007a, Work Instruction for Verification Sampling of the 120-F-1 Glass Dump Waste Site, 0100F-WI-G0069, Rev. 0, Washington Closure Hanford, Richland, Washington.

WCH, 2007b, 100-F Remedial Sampling, Logbook ELF-1174-4, pp. 27-29, 37-39, and 67-68, Washington Closure Hanford, Richland, Washington.

WCH, 2008a, Revised 120-F-1 Verification Sampling, CCN 139173 dated February 14, 2008, to R. Lobos and D.C. Smith from J. M. Capron, Washington Closure Hanford, Richland, Washington.

WCH, 2008b, 100-F Remedial Sampling, Logbook EFL-1173-4 pp 37-39, 81-82, and 88-89, Washington Closure Hanford, Richland, Washington. 


\section{APPENDIX A}

\section{IN-PROCESS AND VERIFICATION SAMPLING RESULTS}


Table A-1 In-Process Samples. (7 pages)

\begin{tabular}{|c|c|c|c|c|c|c|c|c|c|c|c|c|c|c|c|c|c|c|c|c|}
\hline \multirow{2}{*}{ Sample } & \multirow{2}{*}{$\begin{array}{c}\text { HEIS } \\
\text { Number }\end{array}$} & \multirow{2}{*}{ Sample Date } & \multicolumn{3}{|c|}{ Americium-241 GEA } & \multicolumn{3}{|c|}{ Barium-133 } & \multicolumn{3}{|c|}{ Cesium-137 } & \multicolumn{3}{|c|}{ Cobalt- 60} & \multicolumn{3}{|c|}{ Europium-152 } & \multicolumn{3}{|c|}{ Europium-154 } \\
\hline & & & $\mathrm{pCi} / \mathrm{g}$ & $Q$ & MDA & $\mathrm{pCi} / \mathrm{g}$ & $Q$ & MDA & $\mathrm{pCi} / \mathrm{g}$ & $Q$ & MDA & $\mathrm{pCi} / \mathrm{g}$ & $Q$ & MDA & $\mathrm{pCi} / \mathrm{g}$ & $Q$ & MDA & $\mathrm{pCi} / \mathrm{g}$ & $Q$ & MDA \\
\hline soil & $\mathrm{J} 152 \mathrm{H} 3$ & $5 / 3 / 2007$ & 0.088 & $\mathrm{U}$ & 0.088 & & & & 0.06 & $\mathrm{U}$ & 0.06 & 0.069 & $\mathrm{U}$ & 0.069 & 0.14 & $\mathrm{U}$ & 0.14 & 0.2 & $\mathrm{U}$ & 0.2 \\
\hline soil & $\mathrm{J} 152 \mathrm{H} 4$ & $5 / 3 / 2007$ & 0.11 & $\mathrm{U}$ & 0.11 & & & & 0.113 & & 0.038 & 0.035 & $\mathrm{U}$ & 0.035 & 0.091 & $\mathrm{U}$ & 0.091 & 0.12 & $\mathrm{U}$ & 0.12 \\
\hline container & $\mathrm{J} 152 \mathrm{~V} 6$ & $6 / 6 / 2007$ & 0.22 & $\mathrm{U}$ & 0.22 & & & & 0.1 & $\mathrm{U}$ & 0.1 & 0.088 & $\mathrm{U}$ & 0.088 & 0.18 & $\mathrm{U}$ & 0.18 & 0.28 & $\mathrm{U}$ & 0.28 \\
\hline stockpile & J155N6 & $6 / 6 / 2007$ & 0.27 & $\mathrm{U}$ & 0.27 & & & & 0.08 & $\mathrm{U}$ & 0.08 & 0.079 & $\mathrm{U}$ & 0.079 & 0.22 & $\mathrm{U}$ & 0.22 & 0.25 & $\mathrm{U}$ & 0.25 \\
\hline yellow stain & $\mathrm{J} 15 \mathrm{JB} 0$ & $9 / 11 / 2007$ & 0.065 & $U$ & 0.065 & 0.059 & $\mathrm{U}$ & 0.059 & 0.07 & $U$ & 0.07 & 0.067 & $\mathrm{U}$ & 0.067 & 0.165 & $\mathrm{U}$ & 0.165 & 0.249 & $\mathrm{U}$ & 0.249 \\
\hline
\end{tabular}

\begin{tabular}{|c|c|c|c|c|c|c|c|c|c|c|c|c|c|c|c|c|c|c|c|c|}
\hline \multirow{2}{*}{ Sample } & \multirow{2}{*}{$\begin{array}{c}\text { HEIS } \\
\text { Number }\end{array}$} & \multirow{2}{*}{ Sample Date } & \multicolumn{3}{|c|}{ Europium-155 } & \multicolumn{3}{|c|}{ Potassium-40 } & \multicolumn{3}{|c|}{ Radium-226 } & \multicolumn{3}{|c|}{ Radium-228 } & \multicolumn{3}{|c|}{ Silver-108 Metastable } & \multicolumn{3}{|c|}{ Thorium-228 GEA } \\
\hline & & & $\mathrm{pCi} / \mathrm{g}$ & $Q$ & MDA & $\mathrm{pCi} / \mathrm{g}$ & $Q$ & MDA & $\mathrm{pCi} / \mathrm{g}$ & $Q$ & MDA & $\mathrm{pCi} / \mathrm{g}$ & $Q$ & MDA & $\mathrm{pCi} / \mathrm{g}$ & $\mathbf{Q}$ & MDA & $\mathrm{pCi} / \mathrm{g}$ & $Q$ & MDA \\
\hline soil & $\mathrm{J} 152 \mathrm{H} 3$ & $5 / 3 / 2007$ & 0.13 & $\mathrm{U}$ & 0.13 & 12.4 & & 0.38 & 0.414 & & 0.099 & 0.621 & & 0.28 & 0.621 & & 0.28 & 0.578 & & 0.08 \\
\hline soil & $\mathrm{J} 152 \mathrm{H} 4$ & $5 / 3 / 2007$ & 0.09 & $\mathrm{U}$ & 0.09 & 12 & & 0.36 & 0.43 & & 0.063 & 0.758 & & 0.12 & 0.758 & & 0.12 & 0.764 & & 0.056 \\
\hline container & $\mathrm{J} 152 \mathrm{~V} 6$ & $6 / 6 / 2007$ & 0.18 & $\mathrm{U}$ & 0.18 & 10.3 & & 0.84 & 0.379 & & 0.11 & 0.672 & & 0.3 & 0.672 & & 0.3 & 0.46 & & 0.11 \\
\hline stockpile & J155N6 & $6 / 6 / 2007$ & 0.19 & $\mathrm{U}$ & 0.19 & 11.3 & & 0.9 & 0.441 & & 0.13 & 0.575 & & 0.39 & 0.575 & & 0.39 & 0.588 & & 0.12 \\
\hline yellow stain & $\mathrm{J} 15 \mathrm{JB} 0$ & $9 / 11 / 2007$ & 0.142 & $\mathrm{U}$ & 0.142 & 13.2 & & 0.807 & 0.306 & & 0.128 & 0.406 & & 0.278 & 0.406 & & 0.278 & 0.292 & & 0.081 \\
\hline
\end{tabular}

\begin{tabular}{|c|c|c|c|c|c|c|c|c|c|c|c|}
\hline \multirow{2}{*}{ Sample } & \multirow{2}{*}{$\begin{array}{c}\text { HEIS } \\
\text { Number }\end{array}$} & \multirow{2}{*}{ Sample Date } & \multicolumn{3}{|c|}{ Thorium-232 GEA } & \multicolumn{2}{c|}{ Uranium-235 GEA } & \multicolumn{2}{c|}{ Uranium-238 GEA } \\
\cline { 5 - 11 } & & & pCi/g & Q & MDA & pCi/g & Q & MDA & pCi/g & Q & MDA \\
\hline soil & J152H3 & $5 / 3 / 2007$ & 0.621 & & 0.28 & 0.25 & $\mathrm{U}$ & 0.25 & 6.7 & U & 6.7 \\
\hline soil & $\mathrm{J} 152 \mathrm{H} 4$ & $5 / 3 / 2007$ & 0.758 & & 0.12 & 0.15 & $\mathrm{U}$ & 0.15 & 3.8 & $\mathrm{U}$ & 3.8 \\
\hline container & $\mathrm{J} 152 \mathrm{~V} 6$ & $6 / 6 / 2007$ & 0.672 & & 0.3 & 0.27 & $\mathrm{U}$ & 0.27 & 9.5 & $\mathrm{U}$ & 9.5 \\
\hline stockpile & $\mathrm{J} 155 \mathrm{~N} 6$ & $6 / 6 / 2007$ & 0.575 & & 0.39 & 0.31 & $\mathrm{U}$ & 0.31 & 8.7 & $\mathrm{U}$ & 8.7 \\
\hline yellow stain & $\mathrm{J} 15 \mathrm{JB} 0$ & $9 / 11 / 2007$ & 0.406 & & 0.278 & 0.246 & $\mathrm{U}$ & 0.246 & 8.19 & $\mathrm{U}$ & 8.19 \\
\hline
\end{tabular}

\begin{tabular}{|c|c|c|c|c|}
\hline Sample & $\begin{array}{c}\text { HEIS } \\
\text { Number }\end{array}$ & Sample Date & Total Asbestos & Notes \\
\hline suspect ACM & J152H5 & $5 / 3 / 2007$ & non-detected & $60-70 \%$ fiberglass \\
\hline soil & J152H6 & $5 / 3 / 2007$ & non-detected & $3-5 \%$ fiberglass \\
\hline
\end{tabular}

Acronyms and notes apply to all of the tables in this appendix

Note: Data qualified with B, C, and/or J are considered acceptable values.

$\mathrm{C}$ = blank contamination (inorganic compounds)

$\mathrm{D}$ = diluted

$\mathrm{J} \quad=$ estimate value

$\mathrm{PQL}=$ Practical Quantitation Limit

$\mathrm{R}$ = rejected

$\mathrm{Q}=$ qualifier




Table A-1. 120-F-1 In-Process samples. (7 pages)

\begin{tabular}{|c|c|c|c|c|c|c|c|c|c|c|c|c|c|c|c|c|c|}
\hline Sample & $\begin{array}{c}\text { Sample } \\
\text { Number }\end{array}$ & Sample Date & \multicolumn{3}{|c|}{ Aluminum } & \multicolumn{3}{|c|}{ Antimony } & \multicolumn{3}{|c|}{ Arsenic } & \multicolumn{3}{|c|}{ Barium } & \multicolumn{3}{|c|}{ Beryllium } \\
\hline soil & $\mathrm{J} 152 \mathrm{H} 3$ & $5 / 3 / 2007$ & 8910 & & 6.2 & 1.4 & $\bar{U}$ & 1.4 & 1.8 & & 1.8 & 97.4 & $\mathrm{C}$ & 0.09 & 0.53 & & 0.04 \\
\hline soil & $\mathrm{J} 152 \mathrm{H} 4$ & $5 / 3 / 2007$ & 5670 & & 4.6 & 1 & $\mathrm{U}$ & 1 & 2.8 & & 1.3 & 102 & \begin{tabular}{|l|}
$\mathrm{C}$ \\
\end{tabular} & 0.06 & 0.36 & & 0.03 \\
\hline container & J152V6 & $6 / 6 / 2007$ & 3450 & $\mathrm{C}$ & 5.1 & 0.68 & $\mathrm{U}$ & 0.68 & 1.3 & $\mathrm{U}$ & 1.3 & 140 & $\mathrm{C}$ & 0.06 & 0.07 & & 0.03 \\
\hline yellow stain initial & J15JB0 & $9 / 11 / 2007$ & 1280 & $\mathrm{C}$ & 5 & 0.67 & $\mathrm{U}$ & 0.67 & 1.2 & $\bar{U}$ & 1.2 & 48.8 & $\mathrm{C}$ & 0.06 & 0.03 & $\mathrm{U}$ & 0.03 \\
\hline Yellow stain after RTD & $\mathrm{J} 15 \mathrm{P} 45$ & 9/19/2007 & 5350 & & 5.8 & 0.77 & $\mathrm{U}$ & 0.77 & 3.5 & & 1.4 & 46.4 & $\mathrm{C}$ & 0.07 & 0.04 & $\bar{U}$ & 0.04 \\
\hline
\end{tabular}

\begin{tabular}{|c|c|c|c|c|c|c|c|c|c|c|c|c|c|c|c|c|c|}
\hline Sample & $\begin{array}{c}\text { Sample } \\
\text { Number }\end{array}$ & Sample Date & \multicolumn{3}{|c|}{ Boron } & \multicolumn{3}{|c|}{ Cadmium } & \multicolumn{3}{|c|}{ Calcium } & \multicolumn{3}{|c|}{ Chromium (Total) } & \multicolumn{3}{|c|}{ Cobalt } \\
\hline soil & $\mathrm{J} 152 \mathrm{H} 3$ & $5 / 3 / 2007$ & 3.8 & & 1.6 & 0.13 & $\overrightarrow{\mathrm{U}}$ & 0.13 & 10100 & C & 3.2 & 12.1 & $\mathrm{C}$ & 0.34 & 9 & & 0.28 \\
\hline soil & $\mathrm{J} 152 \mathrm{H} 4$ & $5 / 3 / 2007$ & 4.8 & & 1.2 & 0.65 & & 0.09 & 4770 & \begin{tabular}{|l|}
$\mathrm{C}$ \\
\end{tabular} & 2.4 & 8.8 & C & 0.25 & 7 & & 0.28 \\
\hline container & $\mathrm{J} 152 \mathrm{~V} 6$ & $6 / 6 / 2007$ & 20.4 & & 1.1 & 0.23 & & 0.15 & 6950 & $\mathrm{C}$ & 2.2 & 3.1 & & 0.32 & 2.9 & & 0.25 \\
\hline yellow stain initial & J15JB0 & $9 / 11 / 2007$ & 3 & $\mathrm{C}$ & 1.1 & 0.15 & $\overline{\mathrm{U}}$ & 0.15 & 7080 & $\mathrm{C}$ & 2.2 & 3.4 & & 0.3 & 0.59 & & 0.24 \\
\hline Yellow stain after RTD & $\mathrm{J} 15 \mathrm{P} 45$ & $9 / 19 / 2007$ & 1.3 & $\overline{\mathrm{U}}$ & 1.3 & 0.18 & $\overline{\mathrm{U}}$ & 0.18 & 7450 & $\mathrm{C}$ & 2.5 & 15.9 & $\mathrm{C}$ & 0.35 & 2.8 & & 0.28 \\
\hline
\end{tabular}




Table A-1. 120-F-1 In-Process samples. ( 7 pages)

\begin{tabular}{|c|c|c|c|c|c|c|c|c|c|c|c|c|c|c|c|c|c|}
\hline \multirow{2}{*}{ Sample } & \multirow{2}{*}{$\begin{array}{c}\text { Sample } \\
\text { Number } \\
\end{array}$} & \multirow{2}{*}{ Sample Date } & \multicolumn{3}{|c|}{ Manganese } & \multicolumn{3}{|c|}{ Mercury } & \multicolumn{3}{|c|}{ Molybdenum } & \multicolumn{3}{|c|}{ Nickel } & \multicolumn{3}{|c|}{ Potassium } \\
\hline & & & $\mathrm{mg} / \mathrm{kg}$ & Q & $\overline{P Q L}$ & $\mathrm{mg} / \mathrm{kg}$ & $\mathbf{Q}$ & $\mathrm{PQL}$ & $\mathrm{mg} / \mathrm{kg}$ & $\bar{Q}$ & PQL & $\mathrm{mg} / \mathrm{kg}$ & $Q$ & PQL & $\mathrm{mg} / \mathrm{kg}$ & $\bar{Q}$ & PQL \\
\hline soil & $\mathrm{J} 152 \mathrm{H} 3$ & $5 / 3 / 2007$ & 513 & C & 0.09 & 1.3 & & 0.02 & 1 & & 0.55 & 13.2 & & 0.81 & 1480 & & 20.8 \\
\hline soil & $\mathrm{J} 152 \mathrm{H} 4$ & $5 / 3 / 2007$ & 297 & $\mathrm{C}$ & 0.06 & 0.36 & & 0.02 & 5.5 & & 0.41 & 19.6 & & 0.6 & 1330 & & 15.4 \\
\hline container & $\mathrm{J} 152 \mathrm{~V} 6$ & $6 / 6 / 2007$ & 163 & & 0.22 & 0.26 & & 0.01 & 1.2 & & 0.49 & 5.6 & & 0.83 & 639 & & 9.8 \\
\hline stockpile & J155N6 & $5 / 2007$ & 312 & & 0.22 & 0.04 & & 0.02 & 0.5 & $\mathrm{U}$ & 0.5 & 9.9 & & 0.85 & 1210 & & 10 \\
\hline yellow stain initial & $\mathrm{J} 15 \mathrm{JB} 0$ & $9 / 11 / 2007$ & 29.1 & $\mathrm{C}$ & 0.21 & 0.67 & & 0.02 & 0.48 & $\mathrm{U}$ & 0.48 & 0.82 & & 0.82 & 788 & $\mathrm{C}$ & 9.7 \\
\hline Yellow stain after RTD & $\mathrm{J} 15 \mathrm{P} 45$ & $9 / 19 / 2007$ & 118 & & 0.25 & 0.05 & & 0.02 & 0.56 & $\bar{U}$ & 0.56 & 7.4 & & 0.95 & 1190 & $\bar{C}$ & 11.2 \\
\hline
\end{tabular}

\begin{tabular}{|c|c|c|c|c|c|c|c|c|c|c|c|c|c|c|c|c|c|}
\hline \multirow{2}{*}{ Sample } & \multirow{2}{*}{$\begin{array}{c}\text { Sample } \\
\text { Number }\end{array}$} & \multirow{2}{*}{ Sample Date } & \multicolumn{3}{|c|}{ Selenium } & \multicolumn{3}{|c|}{ Silicon } & \multicolumn{3}{|c|}{ Silver } & \multicolumn{3}{|c|}{ Sodium } & \multicolumn{3}{|c|}{ Vanadium } \\
\hline & & & $\mathrm{mg} / \mathrm{kg}$ & $\mathbf{Q}$ & PQL & $\mathrm{mg} / \mathrm{kg}$ & $\mathbf{Q}$ & $\mathrm{PQL}$ & $\mathrm{mg} / \mathrm{kg}$ & $\mathbf{Q}$ & PQL & $\mathrm{mg} / \mathrm{kg}$ & $\mathbf{Q}$ & PQL & $\mathrm{mg} / \mathrm{kg}$ & $\mathbf{Q}$ & $\mathbf{P Q L}$ \\
\hline soil & $\mathrm{J} 152 \mathrm{H} 3$ & $5 / 3 / 2007$ & 1.6 & $\mathrm{U}$ & 1.6 & 1620 & $\mathrm{C}$ & 1.8 & 0.38 & $\mathrm{U}$ & 0.38 & 305 & $\mathrm{C}$ & 1.9 & 63.9 & & 0.43 \\
\hline soil & $\mathrm{J} 152 \mathrm{H} 4$ & $5 / 3 / 2007$ & 1.2 & $\mathrm{U}$ & 1.2 & 541 & $\mathrm{C}$ & 1.3 & 0.51 & & 0.28 & 503 & $\mathrm{C}$ & 1.4 & 46.6 & & 0.32 \\
\hline container & J152V6 & $6 / 6 / 2007$ & 1.3 & $\bar{U}$ & 1.3 & 1200 & & 2.6 & 0.28 & $\mathrm{U}$ & 0.28 & 269 & $\mathrm{C}$ & 2.2 & 16.3 & & 0.25 \\
\hline stockpile & J155N6 & $6 / 6 / 2007$ & 1.3 & $\mathrm{U}$ & 1.3 & 1400 & & 2.7 & 0.28 & $\mathrm{U}$ & 0.28 & 136 & $\mathrm{C}$ & 2.2 & 41.2 & & 0.25 \\
\hline yellow stain initial & J15JB0 & $9 / 11 / 2007$ & 1.3 & $\bar{U}$ & 1.3 & 959 & $\mathrm{C}$ & 2.6 & 0.27 & $\mathrm{U}$ & 0.27 & 678 & $\mathrm{C}$ & 2.1 & 10.2 & & 0.24 \\
\hline Yellow stain after RTD & $\mathrm{J} 15 \mathrm{P} 45$ & 9/19/2007 & 1.5 & $\bar{U}$ & 1.5 & 2650 & $\mathrm{C}$ & 3 & 0.32 & $\mathrm{U}$ & 0.32 & 1040 & $\mathrm{C}$ & 2.5 & 59.4 & & 0.28 \\
\hline
\end{tabular}

\begin{tabular}{|c|c|c|c|c|c|}
\hline \multirow{2}{*}{ Sample } & Sample & \multirow{2}{*}{ Sample Date } & \multicolumn{3}{|c|}{ Zinc } \\
\cline { 4 - 6 } & Number & mg/kg & Q & PQL \\
\hline soil & $\mathrm{J} 152 \mathrm{H} 3$ & $5 / 3 / 2007$ & 61.9 & $\mathrm{C}$ & 0.13 \\
\hline soil & $\mathrm{J} 152 \mathrm{H} 4$ & $5 / 3 / 2007$ & 83.2 & $\mathrm{C}$ & 0.09 \\
\hline container & $\mathrm{J} 152 \mathrm{~V} 6$ & $6 / 6 / 2007$ & 86 & $\mathrm{C}$ & 0.12 \\
\hline stockpile & $\mathrm{J} 155 \mathrm{~N} 6$ & $6 / 6 / 2007$ & 63.5 & $\mathrm{C}$ & 0.13 \\
\hline yellow stain initial & $\mathrm{J} 15 \mathrm{JB} 0$ & $9 / 11 / 2007$ & 10 & $\mathrm{C}$ & 0.12 \\
\hline Yellow stain after RTD & $\mathrm{J} 15 \mathrm{P} 45$ & $9 / 19 / 2007$ & 24 & $\mathrm{C}$ & 0.14 \\
\hline
\end{tabular}


Table A-1. 120-F-1 In-Process Samples. (7 Pages)

\begin{tabular}{|c|c|c|c|c|c|c|c|c|c|c|c|c|c|c|c|c|c|c|}
\hline \multirow[t]{2}{*}{ Constituents } & \multicolumn{3}{|c|}{$\begin{array}{c}\text { Sample J152H3 } \\
\text { soil } \\
5 / 3 / 2007\end{array}$} & \multicolumn{3}{|c|}{$\begin{array}{c}\text { Sample J152H4 } \\
\text { soil } \\
5 / 3 / 2007\end{array}$} & \multicolumn{3}{|c|}{$\begin{array}{c}\text { Sample J152V6 } \\
\text { container } \\
6 / 6 / 2007\end{array}$} & \multicolumn{3}{|c|}{$\begin{array}{c}\text { Sample J155N6 } \\
\text { waste designation } \\
9 / 11 / 07\end{array}$} & \multicolumn{3}{|c|}{$\begin{array}{l}\text { Sample J15JB0 } \\
\text { yellow stain } \\
9 / 11 / 2007\end{array}$} & \multicolumn{3}{|c|}{\begin{tabular}{|c} 
Sample J15P45 yellow \\
stain @ $20 \mathrm{ft} \quad 9 / 19 / 2007$
\end{tabular}} \\
\hline & $\mu \mathrm{g} / \mathrm{kg}$ & Q & PQL & $\mu \mathrm{g} / \mathrm{kg}$ & $\mathbf{Q}$ & $\mathbf{P Q L}$ & $\mu \mathrm{g} / \mathrm{kg}$ & $\mathrm{Q}$ & $\mathbf{P Q L}$ & $\mu \mathrm{g} / \mathrm{kg}$ & $\mathrm{Q}$ & PQL & $\mu \mathrm{g} / \mathrm{kg}$ & $Q$ & $\mathrm{PQL}$ & $\mu \mathrm{g} / \mathrm{kg}$ & $\mathbf{Q}$ & $\mathbf{P Q L}$ \\
\hline \multicolumn{19}{|c|}{ Polychlorinated Biphenyls } \\
\hline Aroclor-1016 & 20 & U & 20 & 220 & UD & 220 & 14 & $U$ & 14 & 14 & $\mathrm{U}$ & 14 & & & & & & \\
\hline Aroclor-1221 & 20 & $U$ & 20 & 220 & UD & 220 & 14 & $\mathrm{U}$ & 14 & 14 & $\mathrm{U}$ & 14 & & & & & & \\
\hline Aroclor-1232 & 20 & $u$ & 20 & 220 & UD & 220 & 14 & 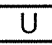 & 14 & 14 & $\mathrm{U}$ & 14 & & & & & & \\
\hline Aroclor-1242 & 20 & $u$ & 20 & 220 & UD & 220 & 14 & $U$ & 14 & 14 & $\mathrm{U}$ & 14 & & & & & & \\
\hline Aroclor-1248 & 20 & U & 20 & 220 & UD & 220 & 14 & $\mathrm{U}$ & 14 & 14 & $\mathrm{U}$ & 14 & & & & & & \\
\hline Aroclor-1254 & 20 & U & 20 & 640 & $\mathrm{D}$ & 220 & 38 & & 14 & 8.3 & $\mathrm{~J}$ & 14 & & & & & & \\
\hline Aroclor-1260 & 20 & $u$ & 20 & 220 & UD & 220 & 14 & $\mathrm{U}$ & 14 & 14 & $\mathrm{U}$ & 14 & & & & & & \\
\hline \multicolumn{19}{|c|}{ Pesticides } \\
\hline Aldrin & & & & & & & & & & & & & 1.4 & UD & 1.4 & 1.6 & UD & 1.6 \\
\hline alpha-BHC & & & & & & & & & & & & & 1.4 & UD & 1.4 & 1.6 & UD & 1.6 \\
\hline alpha-Chlordane & & & & & & & & & & & & & 1.4 & UD & 1.4 & 1.6 & $\mathrm{JD}$ & 1.6 \\
\hline beta-1,2,3,4,5,6-Hexachlorocyclohexane & & & & & & & & & & & & & 1.4 & UD & 1.4 & 1.6 & UD & 1.6 \\
\hline delta-BHC & & & & & & & & & & & & & 1.4 & UD & 1.4 & 1.6 & UD & 1.6 \\
\hline \begin{tabular}{|l|} 
Dichlorodiphenyldichloroethane \\
\end{tabular} & & & & & & & & & & & & & 1.4 & UD & 1.4 & 1.6 & UD & 1.6 \\
\hline Dichlorodiphenyldichloroethylene & & & & & & & & & & & & & 22 & $\mathrm{D}$ & 1.4 & 1.6 & UD & 1.6 \\
\hline Dichlorodiphenyltrichloroethane & & & & & & & & & & & & & 21 & $\mathrm{D}$ & 1.4 & 1.6 & UD & 1.6 \\
\hline Dieldrin & & & & & & & & & & & & & 4.9 & JXD & 1.4 & 1.6 & UD & 1.6 \\
\hline Endosulfan I & & & & & & & & & & & & & 2.4 & $\mathrm{JD}$ & 1.4 & 1.6 & UD & 1.6 \\
\hline Endosulfan II & & & & & & & & & & & & & 3.7 & JXD & 1.4 & 1.6 & UD & 1.6 \\
\hline Endosulfan sulfate & & & & & & & & & & & & & 1.4 & UD & 1.4 & 1.6 & UD & 1.6 \\
\hline Endrin & & & & & & & & & & & & & 1.7 & $J D$ & 1.4 & 1.6 & UD & 1.6 \\
\hline Endrin aldehyde & & & & & & & & & & & & & 4.2 & JXD & 1.4 & 1.6 & UD & 1.6 \\
\hline Endrin ketone & & & & & & & & & & & & & 7 & $\mathrm{XD}$ & 1.4 & 1.6 & UD & 1.6 \\
\hline gamma-BHC (Lindane) & & & & & & & & & & & & & 1.4 & UD & 1.4 & 1.6 & UD & 1.6 \\
\hline $\begin{array}{l}\text { gamma-Chlordane } \\
\end{array}$ & & & & & & & & & & & & & 1.4 & UD & 1.4 & 1.6 & UD & 1.6 \\
\hline Heptachlor & & & & & & & & & & & & & 1.4 & UD & 1.4 & 1.6 & UD & 1.6 \\
\hline Heptachlor epoxide & & & & & & & & & & & & & 1.4 & UD & 1.4 & 1.6 & UD & 1.6 \\
\hline \begin{tabular}{|l} 
Methoxychlor \\
\end{tabular} & & & & & & & & & & & & & 1.4 & UD & 1.4 & 1.6 & UD & 1.6 \\
\hline Toxaphene & & & & & & & & & & & & & 14 & UD & 14 & 16 & UD & 16 \\
\hline
\end{tabular}


Table A-1. 120-F-1 In-Process Samples. (7 Pages)

\begin{tabular}{|c|c|c|c|c|c|c|c|c|c|c|c|c|c|c|c|c|c|c|}
\hline \multirow[t]{2}{*}{ Constituent } & \multicolumn{3}{|c|}{$\begin{array}{c}\text { Sample J152H3 } \\
\text { soil } \\
5 / 3 / 2007\end{array}$} & \multicolumn{3}{|c|}{$\begin{array}{c}\text { Sample J152H4 } \\
\text { soil } \\
5 / 3 / 2007\end{array}$} & \multicolumn{3}{|c|}{$\begin{array}{c}\text { Sample J152V6 } \\
\text { container } \\
6 / 6 / 2007\end{array}$} & \multicolumn{3}{|c|}{$\begin{array}{c}\text { Sample J155N6 } \\
\text { waste designation } \\
9 / 11 / 07\end{array}$} & \multicolumn{3}{|c|}{$\begin{array}{c}\text { Sample J15JB0 } \\
\text { yellow stain } \\
9 / 11 / 2007\end{array}$} & \multicolumn{3}{|c|}{\begin{tabular}{|} 
Sample J15P45 yellow \\
stain @ $20 \mathrm{ft} \quad 9 / 19 / 2007$
\end{tabular}} \\
\hline & $\mu \mathrm{g} / \mathrm{kg}$ & $\mathbf{Q}$ & PQL & $\mu \mathrm{g} / \mathrm{kg}$ & $\mathbf{Q}$ & $\overline{\mathrm{PQL}}$ & $\mu \mathrm{g} / \mathrm{kg}$ & $Q$ & PQL & $\mu \mathrm{g} / \mathrm{kg}$ & $Q$ & PQL & $\mu \mathrm{g} / \mathrm{kg}$ & $Q$ & $\mathbf{P Q L}$ & $\mu \mathrm{g} / \mathrm{kg}$ & $Q$ & PQL \\
\hline \multicolumn{19}{|c|}{ SVOAs } \\
\hline 1,2,4-Trichlorobenzene & 500 & U & 500 & 370 & $U$ & 370 & 360 & $\mathrm{U}$ & 360 & 360 & $U$ & 360 & & & & & & \\
\hline 1,2-Dichlorobenzene & 500 & $\mathrm{U}$ & 500 & 370 & $\mathrm{U}$ & 370 & 360 & $U$ & 360 & 360 & $\mathrm{U}$ & 360 & & & & & & \\
\hline 1,3-Dichlorobenzene & 500 & $\mathrm{U}$ & 500 & 370 & $U$ & 370 & 360 & $U$ & 360 & 360 & $\mathrm{U}$ & 360 & & & & & & \\
\hline \begin{tabular}{|l}
$1,4-D i c h l o r o b e n z e n e$ \\
\end{tabular} & 500 & $\mathrm{U}$ & 500 & 370 & $U$ & 370 & 360 & $U$ & 360 & 360 & $\mathrm{U}$ & 360 & & & & & & \\
\hline 2,4,5-Trichlorophenol & 1300 & $\mathrm{U}$ & 1300 & 930 & $\mathrm{U}$ & 930 & 900 & $\mathrm{U}$ & 900 & 890 & $\mathrm{U}$ & 890 & & & & & & \\
\hline 2,4,6-Trichlorophenol & 500 & $\mathrm{U}$ & 500 & 370 & U & 370 & 360 & $U$ & 360 & 360 & $U$ & 360 & & & & & & \\
\hline 2,4-Dichlorophenol & 500 & $\mathrm{U}$ & 500 & 370 & $\mathrm{U}$ & 370 & 360 & $U$ & 360 & 360 & $U$ & 360 & & & & & & \\
\hline 2,4-Dimethylphenol & 500 & $\mathrm{U}$ & 500 & 370 & $\mathrm{U}$ & 370 & 360 & $\mathrm{U}$ & 360 & 360 & $\mathrm{U}$ & 360 & & & & & & \\
\hline 2,4-Dinitrophenol & 1300 & $U$ & 1300 & 930 & $\mathrm{U}$ & 930 & 900 & $\mathrm{U}$ & 900 & 890 & $\mathrm{U}$ & 890 & & & & & & \\
\hline 2,4-Dinitrotoluene & 500 & $\mathrm{U}$ & 500 & 370 & $U$ & 370 & 360 & $\mathrm{U}$ & 360 & 360 & $U$ & 360 & & & & & & \\
\hline 2,6-Dinitrotoluene & 500 & $\mathrm{U}$ & 500 & 370 & $U$ & 370 & 360 & $U$ & 360 & 360 & $\mathrm{U}$ & 360 & & & & & & \\
\hline 2-Chloronaphthalene & 500 & $\mathrm{U}$ & 500 & 370 & $U$ & 370 & 360 & $\mathrm{U}$ & 360 & 360 & $\mathrm{U}$ & 360 & & & & & & \\
\hline 2-Chlorophenol & 500 & $\mathrm{U}$ & 500 & 370 & U & 370 & 360 & $\mathrm{U}$ & 360 & 360 & $\mathrm{U}$ & 360 & & & & & & \\
\hline 2-Methylnaphthalene & 500 & $U$ & 500 & 65 & J & 370 & 360 & $\mathrm{U}$ & 360 & 360 & $\mathrm{U}$ & 360 & & & & & & \\
\hline 2-Methylphenol (cresol, o-) & 500 & $U$ & 500 & 370 & $\mathrm{U}$ & 370 & 360 & $U$ & 360 & 360 & $\mathrm{U}$ & 360 & & & & & & \\
\hline 2-Nitroaniline & 1300 & $\mathrm{U}$ & 1300 & 930 & U & 930 & 900 & $U$ & 900 & 890 & $U$ & 890 & & & & & & \\
\hline 2-Nitrophenol & 500 & $U$ & 500 & 370 & $\mathrm{U}$ & 370 & 360 & $U$ & 360 & 360 & $U$ & 360 & & & & & & \\
\hline 3,3'-Dichlorobenzidine & 500 & $\mathrm{U}$ & 500 & 370 & $\mathrm{U}$ & 370 & 360 & $U$ & 360 & 360 & $\mathrm{U}$ & 360 & & & & & & \\
\hline \begin{tabular}{|l} 
3-Nitroaniline \\
\end{tabular} & 500 & $\mathrm{U}$ & 500 & 370 & $\mathrm{U}$ & 370 & 360 & $U$ & 360 & 360 & $\mathrm{U}$ & 360 & & & & & & \\
\hline \begin{tabular}{|l|} 
4,6-Dinitro-2-methylphenol \\
\end{tabular} & 1300 & $\mathrm{U}$ & 1300 & 930 & $\mathrm{U}$ & 930 & 900 & $U$ & 900 & 890 & $\mathrm{U}$ & 890 & & & & & & \\
\hline \begin{tabular}{|l} 
4-Bromophenylphenyl ether \\
\end{tabular} & 1300 & $U$ & 1300 & 930 & $\mathrm{U}$ & 930 & 900 & $U$ & 900 & 890 & $\mathrm{U}$ & 890 & & & & & & \\
\hline \begin{tabular}{|l} 
4-Chloro-3-methylphenol \\
\end{tabular} & 500 & $\mathrm{U}$ & 500 & 370 & $\mathrm{U}$ & 370 & 360 & $U$ & 360 & 360 & $\mathrm{U}$ & 360 & & & & & & \\
\hline 4-Chloroaniline & 500 & $\mathrm{U}$ & 500 & 370 & $\mathrm{U}$ & 370 & 360 & $U$ & 360 & 360 & $\mathrm{U}$ & 360 & & & & & & \\
\hline 4-Chlorophenylphenyl ether & 500 & $\mathrm{U}$ & 500 & 370 & $\mathrm{U}$ & 370 & 360 & $\mathrm{U}$ & 360 & 360 & $\mathrm{U}$ & 360 & & & & & & \\
\hline 4-Methylphenol (cresol, p-) & 500 & $\mathrm{U}$ & 500 & 370 & $\mathrm{U}$ & 370 & 360 & $U$ & 360 & 360 & $U$ & 360 & & & & & & \\
\hline 4-Nitroaniline & 1300 & $\mathrm{U}$ & 1300 & 930 & $U$ & 930 & 900 & $\mathrm{U}$ & 900 & 890 & $\mathrm{U}$ & 890 & & & & & & \\
\hline 4-Nitrophenol & 1300 & $\mathrm{U}$ & 1300 & 930 & $\mathrm{U}$ & 930 & 900 & $\mathrm{U}$ & 900 & 890 & $\mathrm{U}$ & 890 & & & & & & \\
\hline \begin{tabular}{|l|} 
Acenaphthene \\
\end{tabular} & 500 & $\mathrm{U}$ & 500 & 370 & $\mathrm{U}$ & 370 & 360 & $\mathrm{U}$ & 360 & 360 & $\mathrm{U}$ & 360 & & & & & & \\
\hline \begin{tabular}{|l} 
Acenaphthylene \\
\end{tabular} & 500 & $\mathrm{U}$ & 500 & 370 & $\mathrm{U}$ & 370 & 360 & $\mathrm{U}$ & 360 & 360 & $\mathrm{U}$ & 360 & & & & & & \\
\hline \begin{tabular}{|l} 
Anthracene \\
\end{tabular} & 500 & $\mathrm{U}$ & 500 & 580 & & 370 & 360 & $\mathrm{U}$ & 360 & 360 & $\mathrm{U}$ & 360 & & & & & & \\
\hline Benzo(a)anthracene & 500 & $\mathrm{U}$ & 500 & 42 & $\mathrm{~J}$ & 370 & 41 & $J$ & 360 & 360 & $\mathrm{U}$ & 360 & & & & & & \\
\hline \begin{tabular}{|l|} 
Benzo(a)pyrene \\
\end{tabular} & 500 & $\mathrm{U}$ & 500 & 370 & $\mathrm{U}$ & 370 & 65 & $J$ & 360 & 30 & $\mathrm{~J}$ & 360 & & & & & & \\
\hline Benzo(b)fluoranthene & 500 & $\mathrm{U}$ & 500 & 370 & $\mathrm{U}$ & 370 & 63 & $\mathrm{~J}$ & 360 & 31 & $\mathrm{~J}$ & 360 & & & & & & \\
\hline Benzo(ghi)perylene & 500 & U & 500 & 370 & $\mathrm{U}$ & 370 & 47 & $\mathrm{~J}$ & 360 & 39 & $\mathrm{~J}$ & 360 & & & & & & \\
\hline Benzo(k)fluoranthene & 500 & $\mathrm{U}$ & 500 & 370 & $\mathrm{U}$ & 370 & 69 & $\mathrm{~J}$ & 360 & 35 & $\mathrm{~J}$ & 360 & & & & & & \\
\hline Bis(2-chloro-1-methylethyl)ether & 500 & $\mathrm{U}$ & 500 & 370 & $\mathrm{U}$ & 370 & 360 & $U$ & 360 & 360 & $\mathrm{U}$ & 360 & & & & & & \\
\hline Bis(2-Chloroethoxy)methane & 500 & U & 500 & 370 & $\mathrm{U}$ & 370 & 360 & $\mathrm{U}$ & 360 & 360 & $\mathrm{U}$ & 360 & & & & & & \\
\hline
\end{tabular}


Table A-1. 120-F-1 In-Process Samples. (7 Pages)

\begin{tabular}{|c|c|c|c|c|c|c|c|c|c|c|c|c|c|c|c|c|c|c|}
\hline \multirow[t]{2}{*}{ Constituent } & \multicolumn{3}{|c|}{$\begin{array}{l}\text { Sample J152H3 } \\
\text { soil } \\
5 / 3 / 2007\end{array}$} & \multicolumn{3}{|c|}{$\begin{array}{c}\text { Sample J152H4 } \\
\text { soil } \\
5 / 3 / 2007\end{array}$} & \multicolumn{3}{|c|}{$\begin{array}{l}\text { Sample J152V6 } \\
\text { container } \\
6 / 6 / 2007\end{array}$} & \multicolumn{3}{|c|}{$\begin{array}{c}\text { Sample J155N6 } \\
\text { waste designation } \\
9 / 11 / 07\end{array}$} & \multicolumn{3}{|c|}{$\begin{array}{l}\text { Sample J15JB0 } \\
\text { yellow stain } \\
\text { 9/11/2007 }\end{array}$} & \multicolumn{3}{|c|}{$\begin{array}{l}\text { Sample J15P45 yellow } \\
\text { stain @ } 20 \mathrm{ft} \quad 9 / 19 / 2007\end{array}$} \\
\hline & $\mu \mathrm{g} / \mathrm{kg}$ & \begin{tabular}{|l|l|l|} 
Q & \\
\end{tabular} & $\mathrm{PQL}$ & $\mu \mathrm{g} / \mathrm{kg}$ & $\mathrm{Q}$ & PQL & $\mu \mathrm{g} / \mathrm{kg}$ & $Q$ & $\mathrm{PQL}$ & $\mu \mathrm{g} / \mathrm{kg}$ & $Q$ & $\mathrm{PQL}$ & $\mu \mathrm{g} / \mathrm{kg}$ & $\mathrm{Q}$ & PQL & $\mu \mathrm{g} / \mathrm{kg}$ & $\mathrm{Q}$ & PQL \\
\hline \multicolumn{19}{|c|}{ SVOAs (continued) } \\
\hline \begin{tabular}{|l} 
Bis(2-chloroethyl) ether \\
\end{tabular} & 500 & U & 500 & 370 & $\mathrm{U}$ & 370 & 360 & $U$ & 360 & 360 & $\mathrm{U}$ & 360 & & & & & & \\
\hline Bis(2-ethylhexyl) phthalate & 110 & \begin{tabular}{|l|l|l|l|l|l|l|l|l}
$J$ & \\
\end{tabular} & 500 & 140 & $\mathrm{~J}$ & 370 & 180 & $\mathrm{JB}$ & 360 & 72 & $\mathrm{JB}$ & 360 & & & & & & \\
\hline \begin{tabular}{|l} 
Butylbenzylphthalate \\
\end{tabular} & 500 & \begin{tabular}{|l|l} 
\\
\end{tabular} & 500 & 370 & $\mathrm{U}$ & 370 & 360 & $U$ & 360 & 360 & U & 360 & & & & & & \\
\hline \begin{tabular}{|l|} 
Carbazole \\
\end{tabular} & 500 & \begin{tabular}{|l|l|} 
\\
\end{tabular} & 500 & 370 & $\mathrm{U}$ & 370 & 360 & $U$ & 360 & 360 & $\mathrm{U}$ & 360 & & & & & & \\
\hline \begin{tabular}{|l|} 
Chrysene \\
\end{tabular} & 500 & \begin{tabular}{|l|l}
$U$ \\
\end{tabular} & 500 & 99 & $\mathrm{~J}$ & 370 & 86 & $\mathrm{~J}$ & 360 & 24 & \begin{tabular}{|l|l|}
$J$ \\
\end{tabular} & 360 & & & & & & \\
\hline Di-n-butylphthalate & 45 & \begin{tabular}{|l|l|l|} 
\\
\end{tabular} & 500 & 370 & $\mathrm{U}$ & 370 & 27 & $\mathrm{JB}$ & 360 & 48 & JB & 360 & & & & & & \\
\hline \begin{tabular}{|l|} 
Di-n-octylphthalate \\
\end{tabular} & 500 & \begin{tabular}{|l|l|}
$U$ \\
\end{tabular} & 500 & 370 & $U$ & 370 & 360 & $\mathrm{U}$ & 360 & 360 & $\mathrm{U}$ & 360 & & & & & & \\
\hline Dibenz[a,h]anthracene & 500 & U & 500 & 370 & $\mathrm{U}$ & 370 & 360 & $\mathrm{U}$ & 360 & 360 & $\mathrm{U}$ & 360 & & & & & & \\
\hline \begin{tabular}{|l} 
Dibenzofuran \\
\end{tabular} & 500 & U & 500 & 370 & $\mathrm{U}$ & 370 & 360 & $\mathrm{U}$ & 360 & 360 & $\mathrm{U}$ & 360 & & & & & & \\
\hline Diethylphthalate & 500 & U & 500 & 370 & $\mathrm{U}$ & 370 & 360 & $\mathrm{U}$ & 360 & 360 & $\mathrm{U}$ & 360 & & & & & & \\
\hline \begin{tabular}{|l} 
Dimethyl phthalate \\
\end{tabular} & 500 & u & 500 & 370 & $\mathrm{U}$ & 370 & 360 & $\mathrm{U}$ & 360 & 360 & $\mathrm{U}$ & 360 & & & & & & \\
\hline \begin{tabular}{|l} 
Fluoranthene \\
\end{tabular} & 500 & U & 500 & 71 & $\mathrm{~J}$ & 370 & 63 & $\mathrm{~J}$ & 360 & 28 & $\mathrm{~J}$ & 360 & & & & & & \\
\hline \begin{tabular}{|l} 
Fluorene \\
\end{tabular} & 500 & U & 500 & 370 & $\mathrm{U}$ & 370 & 360 & $\bar{U}$ & 360 & 360 & $U$ & 360 & & & & & & \\
\hline \begin{tabular}{|l|} 
Hexachlorobenzene \\
\end{tabular} & 500 & u & 500 & 370 & $U$ & 370 & 360 & $\bar{U}$ & 360 & 360 & $\mathrm{U}$ & 360 & & & & & & \\
\hline Hexachlorobutadiene & 500 & u & 500 & 370 & $\mathrm{U}$ & 370 & 360 & $U$ & 360 & 360 & $U$ & 360 & & & & & & \\
\hline Hexachlorocyclopentadiene & 500 & U & 500 & 370 & $\mathrm{U}$ & 370 & 360 & $U$ & 360 & 360 & $\mathrm{U}$ & 360 & & & & & & \\
\hline Hexachloroethane & 500 & $\mathrm{u}$ & 500 & 370 & $\mathrm{U}$ & 370 & 360 & $\mathrm{U}$ & 360 & 360 & $U$ & 360 & & & & & & \\
\hline Indeno( $(1,2,3$-cd)pyrene & 500 & $\mathrm{U}$ & 500 & 370 & $\mathrm{U}$ & 370 & 43 & $J$ & 360 & 35 & $\mathrm{~J}$ & 360 & & & & & & \\
\hline \begin{tabular}{|l|} 
Isophorone \\
\end{tabular} & 500 & U & 500 & 370 & $\mathrm{U}$ & 370 & 360 & $\bar{U}$ & 360 & 360 & $\mathrm{U}$ & 360 & & & & & & \\
\hline N-Nitroso-di-n-dipropylamine & 500 & 0 & 500 & 370 & $\mathrm{U}$ & 370 & 360 & $\mathrm{U}$ & 360 & 360 & $\mathrm{U}$ & 360 & & & & & & \\
\hline N-Nitrosodiphenylamine & 500 & $\mathrm{U}$ & 500 & 370 & $\mathrm{U}$ & 370 & 360 & $\mathrm{U}$ & 360 & 360 & $\mathrm{U}$ & 360 & & & & & & \\
\hline \begin{tabular}{|l|} 
Naphthalene \\
\end{tabular} & 500 & 0 & 500 & 59 & $\mathrm{~J}$ & 370 & 360 & $\bar{U}$ & 360 & 360 & $U$ & 360 & & & & & & \\
\hline \begin{tabular}{|l|} 
Nitrobenzene \\
\end{tabular} & 500 & U & 500 & 370 & $\mathrm{U}$ & 370 & 360 & $u$ & 360 & 360 & $\mathrm{U}$ & 360 & & & & & & \\
\hline Pentachlorophenol & 1300 & U & 1300 & 20000 & $\mathrm{D}$ & 9300 & 34 & $\mathrm{~J}$ & 900 & 890 & $\mathrm{U}$ & 890 & & & & & & \\
\hline Phenanthrene & 500 & 0 & 500 & 370 & $\mathrm{U}$ & 370 & 33 & $\mathrm{~J}$ & 360 & 18 & $\mathrm{~J}$ & 360 & & & & & & \\
\hline Phenol & 500 & u & 500 & 370 & $\mathrm{U}$ & 370 & 48 & 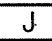 & 360 & 360 & $U$ & 360 & & & & & & \\
\hline Pyrene & 500 & U & 500 & 170 & $\mathrm{~J}$ & 170 & 100.255 & $\mathrm{~J}$ & 360 & 40 & $\mathrm{~J}$ & 360 & & & & & & \\
\hline \multicolumn{19}{|c|}{ Total Petroleum Hydrocarbon (TPH) } \\
\hline
\end{tabular}


Table A-1. 120-F-1 In-Process Samples. (7 Pages)

\begin{tabular}{|c|c|c|c|c|c|c|c|c|c|c|c|c|c|c|c|c|c|c|}
\hline \multirow[t]{2}{*}{ Constituent } & \multicolumn{3}{|c|}{$\begin{array}{c}\text { Sample J152H3 } \\
\text { soil } \\
5 / 3 / 2007\end{array}$} & \multicolumn{3}{|c|}{$\begin{array}{c}\text { Sample J152H4 } \\
\text { soil } \\
5 / 3 / 2007\end{array}$} & \multicolumn{3}{|c|}{$\begin{array}{c}\text { Sample J152V6 } \\
\text { container } \\
6 / 6 / 2007\end{array}$} & \multicolumn{3}{|c|}{$\begin{array}{c}\text { Sample J155N6 } \\
\text { waste designation } \\
9 / 11 / 07\end{array}$} & \multicolumn{3}{|c|}{$\begin{array}{c}\text { Sample J15JB0 } \\
\text { yellow stain } \\
9 / 11 / 2007\end{array}$} & \multicolumn{3}{|c|}{$\begin{array}{l}\text { Sample J15P45 yellow } \\
\text { stain @ } 20 \mathrm{ft} \quad 9 / 19 / 2007\end{array}$} \\
\hline & $\mu \mathrm{g} / \mathrm{kg}$ & Q & PQL & $\mu \mathrm{g} / \mathrm{kg}$ & $Q$ & PQL & $\mu \mathrm{g} / \mathrm{kg}$ & $\mathrm{Q}$ & $\overline{\text { PQL }}$ & $\mu \mathrm{g} / \mathrm{kg}$ & $\mathbf{Q}$ & PQL & $\mu \mathrm{g} / \mathrm{kg}$ & $Q$ & PQL & $\mu \mathrm{g} / \mathrm{kg}$ & $\mathrm{Q}$ & PQL \\
\hline \multicolumn{19}{|c|}{$\frac{1 \text { pons }}{\text { VOAs }}$} \\
\hline 1,1,1-Trichloroethane & & & & & & & 6 & $U$ & 6 & 6 & $U$ & 6 & & & & & & \\
\hline $1,1,2,2$-Tetrachloroethane & & & & & & & 6 & $\bar{U}$ & 6 & 6 & $\mathrm{U}$ & 6 & & & & & & \\
\hline 1,1,2-Trichloroethane & & & & & & & 6 & $\bar{U}$ & 6 & 6 & $U$ & 6 & & & & & & \\
\hline 1,1-Dichloroethane & & & & & & & 6 & $\mathrm{U}$ & 6 & 6 & $U$ & 6 & & & & & & \\
\hline 1,1-Dichloroethene & & & & & & & 6 & $U$ & 6 & 6 & $U$ & 6 & & & & & & \\
\hline 1,2-Dichloroethane & & & & & & & 6 & $\mathrm{U}$ & 6 & 6 & $\mathrm{U}$ & 6 & & & & & & \\
\hline 1,2-Dichloroethene(Total) & & & & & & & 6 & $\mathrm{U}$ & 6 & 6 & $U$ & 6 & & & & & & \\
\hline 1,2-Dichloropropane & & & & & & & 6 & $\mathrm{U}$ & 6 & 6 & $U$ & 6 & & & & & & \\
\hline 2-Butanone & & & & & & & 11 & $U$ & 11 & 11 & $U$ & 11 & & & & & & \\
\hline 2-Hexanone & & & & & & & 11 & $U$ & 11 & 11 & $U$ & 11 & & & & & & \\
\hline \begin{tabular}{|l} 
4-Methyl-2-Pentanone \\
\end{tabular} & & & & & & & 11 & $\mathrm{U}$ & 11 & 11 & $U$ & 11 & & & & & & \\
\hline Acetone & & & & & & & 11 & $\mathrm{U}$ & 11 & 2 & J & 11 & & & & & & \\
\hline Benzene & & & & & & & 6 & $\mathrm{U}$ & 6 & $\frac{2}{6}$ & $\mathrm{U}$ & 6 & & & & & & \\
\hline Bromodichloromethane & & & & & & & 6 & $U$ & 6 & 6 & $U$ & 6 & & & & & & \\
\hline Bromoform & & & & & & & 6 & $\mathrm{U}$ & 6 & 6 & $U$ & 6 & & & & & & \\
\hline Bromomethane & & & & & & & 11 & $\mathrm{U}$ & 11 & 11 & $U$ & 11 & & & & & & \\
\hline Carbon disulfide & & & & & & & 6 & $U$ & 6 & 6 & $U$ & 6 & & & & & & \\
\hline Carbon tetrachloride & & & & & & & 6 & $U$ & 6 & $\frac{0}{6}$ & $\mathrm{U}$ & $\frac{0}{6}$ & & & & & & \\
\hline \begin{tabular}{|l} 
Chlorobenzene \\
\end{tabular} & & & & & & & $\frac{0}{6}$ & $\mathrm{U}$ & $\frac{0}{6}$ & 6 & $U$ & 6 & & & & & & \\
\hline \begin{tabular}{|l} 
Chloroethane \\
\end{tabular} & & & & & & & 11 & $U$ & $\frac{0}{11}$ & 11 & $\bar{U}$ & $\frac{0}{11}$ & & & & & & \\
\hline Chloroform & & & & & & & 6 & $U$ & $\frac{11}{6}$ & 6 & $U$ & $\frac{11}{6}$ & & & & & & \\
\hline \begin{tabular}{|l} 
Chloromethane \\
\end{tabular} & & & & & & & 11 & $\mathrm{U}$ & 11 & 11 & 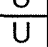 & $\frac{0}{6}$ & & & & & & \\
\hline cis-1,2-Dichloroethylene & & & & & & & 6 & $\mathrm{U}$ & $\frac{11}{6}$ & 6 & 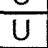 & 6 & & & & & & \\
\hline cis-1,3-Dichloropropene & & & & & & & 6 & $\mathrm{U}$ & 6 & 6 & $U$ & 6 & & & & & & \\
\hline \begin{tabular}{|l} 
Dibromochloromethane \\
\end{tabular} & & & & & & & 6 & $\mathrm{U}$ & 6 & 6 & $U$ & 6 & & & & & & \\
\hline \begin{tabular}{|l} 
Ethylbenzene \\
\end{tabular} & & & & & & & $\frac{0}{6}$ & $U$ & $\frac{0}{6}$ & $\frac{0}{6}$ & $U$ & $\frac{0}{6}$ & & & & & & \\
\hline \begin{tabular}{|l} 
Methylenechloride \\
\end{tabular} & & & & & & & 11 & $\mathrm{~B}$ & $\frac{0}{6}$ & $\frac{0}{9}$ & $\mathrm{~B}$ & $\frac{0}{6}$ & & & & & & \\
\hline Styrene & & & & & & & 6 & $\mathrm{U}$ & 6 & 6 & $U$ & 6 & & & & & & \\
\hline Tetrachloroethene & & & & & & & 6 & $U$ & 6 & 6 & $U$ & 6 & & & & & & \\
\hline Toluene & & & & & & & 6 & $\mathrm{U}$ & 6 & 6 & $\bar{U}$ & 6 & & & & & & \\
\hline trans-1,2-Dichloroethylene & & & & & & & 6 & $\mathrm{U}$ & 6 & 6 & 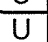 & 6 & & & & & & \\
\hline \begin{tabular}{|l} 
trans-1,3-Dichloropropene \\
\end{tabular} & & & & & & & 6 & $\mathrm{U}$ & 6 & 6 & $\mathrm{U}$ & 6 & & & & & & \\
\hline Trichloroethene & & & & & & & 6 & $U$ & 6 & 6 & $\mathrm{U}$ & 6 & & & & & & \\
\hline \begin{tabular}{|l} 
Vinyl chloride \\
\end{tabular} & & & & & & & 11 & $U$ & 11 & 11 & $U$ & 11 & & & & & & \\
\hline Xylenes (total) & & & & & & & 6 & $\mathrm{U}$ & 6 & 6 & $U$ & 6 & & & & & & \\
\hline
\end{tabular}


Table A-2. 120-F-1 Northwest Excavation Sampling Results from Initial Verification Samples. (8 Pages)

\begin{tabular}{|c|c|c|c|c|c|c|c|c|c|c|c|c|c|c|c|c|c|c|c|c|c|c|c|}
\hline \multirow{2}{*}{$\begin{array}{c}\text { Sample } \\
\text { Location }\end{array}$} & \multirow{2}{*}{$\begin{array}{c}\text { HEIS } \\
\text { Number }\end{array}$} & \multirow{2}{*}{$\begin{array}{c}\text { Sample } \\
\text { Date }\end{array}$} & \multicolumn{3}{|c|}{ Aluminum } & \multicolumn{3}{|c|}{ Antimony } & \multicolumn{3}{|c|}{ Arsenic } & \multicolumn{3}{|c|}{ Barium } & \multicolumn{3}{|c|}{ Beryllium } & \multicolumn{3}{|c|}{ Boron } & \multicolumn{3}{|c|}{ Cadmium } \\
\hline & & & $\mathrm{mg} / \mathrm{kg}$ & $Q$ & $\mathrm{PQL}$ & $\mathrm{mg} / \mathrm{kg}$ & $Q$ & $\mathrm{PQL}$ & $\mathrm{mg} / \mathrm{kg}$ & $Q$ & $\mathrm{PQL}$ & $\mathrm{mg} / \mathrm{kg}$ & Q & $\mathrm{PQL}$ & $\mathrm{mg} / \mathrm{kg}$ & $Q$ & $\mathrm{PQL}$ & $\mathrm{mg} / \mathrm{kg}$ & Q & $\mathrm{PQL}$ & $\mathrm{mg} / \mathrm{kg}$ & Q & $\overline{P Q L}$ \\
\hline NW-1 & $\mathrm{J} 16343$ & $12 / 3 / 2007$ & 5490 & & 11.2 & 0.84 & $\mathrm{U}$ & 0.84 & 2.1 & & 1.4 & 49.8 & & 0.28 & 0.39 & & 0.14 & 1.4 & \begin{tabular}{|l|} 
\\
\end{tabular} & 1.4 & 0.14 & $\mathrm{U}$ & 0.14 \\
\hline NW-2 & J16344 & $12 / 3 / 2007$ & 6500 & & 12.4 & 0.93 & $\mathrm{U}$ & 0.93 & 2.2 & & 1.5 & 77 & & 0.31 & 0.44 & & 0.15 & 4.7 & & 1.5 & 0.15 & $\mathrm{U}$ & 0.15 \\
\hline NW-3 & $\mathrm{J} 16345$ & $12 / 3 / 2007$ & 5000 & & 11.5 & 0.87 & $\mathrm{U}$ & 0.87 & 2.6 & & 1.4 & 65.7 & & 0.29 & 0.36 & & 0.14 & 2.3 & & 1.4 & 0.14 & \begin{tabular}{|l|}
$\mathrm{U}$ \\
\end{tabular} & 0.14 \\
\hline NW-4 & $\mathrm{J} 16346$ & $12 / 3 / 2007$ & 5780 & & 12.5 & 0.94 & $\mathrm{U}$ & 0.94 & 2.6 & & 1.6 & 79.5 & & 0.31 & 0.4 & & 0.16 & 2.7 & & 1.6 & 0.16 & $\mathrm{U}$ & 0.16 \\
\hline NW-5 & $\mathrm{J} 16347$ & $12 / 3 / 2007$ & 5500 & & 12.8 & 0.96 & $\mathrm{U}$ & 0.96 & 2.1 & & 1.6 & 65.8 & & 0.32 & 0.37 & & 0.16 & 1.9 & & 1.6 & 0.16 & $\mathrm{U}$ & 0.16 \\
\hline$N W-6$ & $\mathrm{~J} 16348$ & $12 / 3 / 2007$ & 5790 & & 11.7 & 0.88 & $\mathrm{U}$ & 0.88 & 2.3 & & 1.5 & 64.1 & & 0.29 & 0.33 & & 0.15 & 2 & & 1.5 & 0.55 & & 0.15 \\
\hline NW-7 & $J 16349$ & $12 / 3 / 2007$ & 4600 & & 11.7 & 0.88 & $\mathrm{U}$ & 0.88 & 2.3 & & 1.5 & 81.3 & & 0.29 & 0.3 & & 0.15 & 2.3 & & 1.5 & 1.0 & & 0.15 \\
\hline Dup of & & & & & & & & & & & & & & & & & & & & & & & \\
\hline $\mathrm{J} 16349$ & $\mathrm{~J} 16350$ & $12 / 3 / 2007$ & 5340 & & 12.2 & 0.92 & $\mathrm{U}$ & 0.92 & 2.4 & & 1.5 & 72.3 & & 0.31 & 0.31 & & 0.15 & 1.9 & & 1.5 & 0.49 & & 0.15 \\
\hline NW-8 & $\mathrm{J} 16351$ & $12 / 3 / 2007$ & 4780 & & 12.4 & 0.93 & $\mathrm{U}$ & 0.93 & 2.6 & & 1.5 & 48.1 & & 0.31 & 0.29 & & 0.15 & 1.7 & & 1.5 & 0.71 & & 0.15 \\
\hline NW-9 & $\mathrm{J} 16352$ & $12 / 3 / 2007$ & 5090 & & 12.3 & 0.92 & $\mathrm{U}$ & 0.92 & 3 & & 1.5 & 67.2 & & 0.31 & 0.33 & & 0.15 & 1.6 & & 1.5 & 0.15 & $\mathrm{U}$ & 0.15 \\
\hline NW-10 & $\mathrm{J16353}$ & $12 / 3 / 2007$ & 4930 & & 12.2 & 0.92 & $\mathrm{U}$ & 0.92 & 2.3 & & 1.5 & 59.8 & & 0.31 & 0.33 & & 0.15 & 1.5 & $\mathrm{U}$ & 1.5 & 0.15 & $\mathrm{U}$ & 0.15 \\
\hline Equip blank & $\mathrm{J16354}$ & $12 / 17 / 2007$ & 80.7 & & 3.7 & 0.28 & $\mathrm{U}$ & 0.28 & 0.46 & $\mathrm{U}$ & 0.46 & 1.7 & & 0.09 & 0.05 & $\mathrm{U}$ & 0.05 & 0.46 & \begin{tabular}{|l|}
$\mathrm{U}$ \\
\end{tabular} & 0.46 & 0.05 & $\mathrm{U}$ & 0.05 \\
\hline anomaly & $\mathrm{J} 163 \mathrm{~T} 2$ & $12 / 11 / 2007$ & 114 & & 3.6 & 1.6 & $\mathrm{U}$ & 0.27 & 2.8 & $\mathrm{U}$ & 0.45 & 1.8 & & 0.09 & 0.04 & & 0.04 & 0.36 & C & 0.45 & 0.50 & $U$ & 0.04 \\
\hline
\end{tabular}



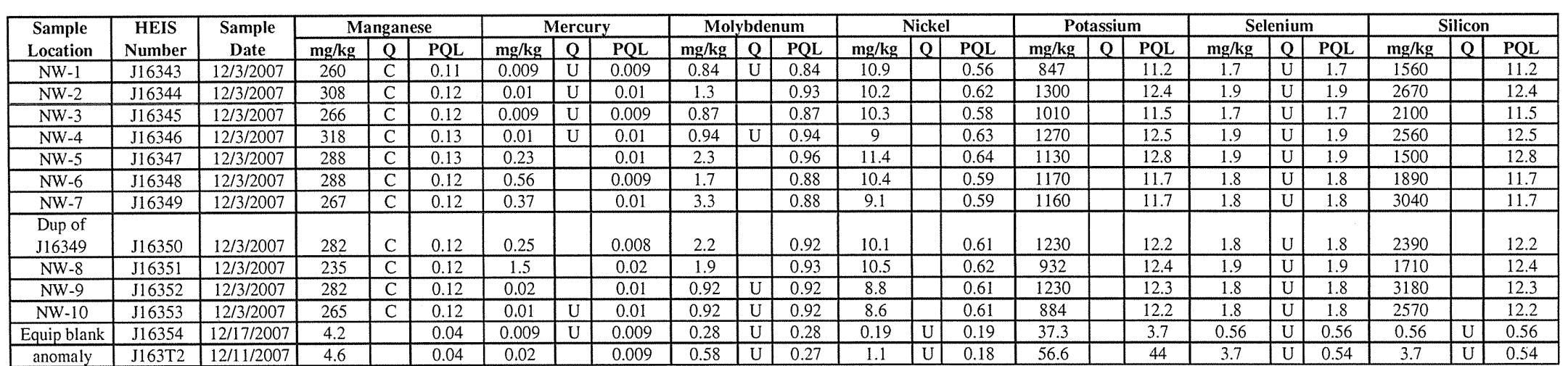


Table A-2. 120-F-1 Northwest Excavation Sampling Results from Initial Verification Samples. (8 Pages)

\begin{tabular}{|c|c|c|c|c|c|c|c|c|c|c|c|c|c|c|c|c|c|c|c|c|c|c|c|}
\hline \multirow{2}{*}{$\begin{array}{l}\text { Sample } \\
\text { Location }\end{array}$} & \multirow{2}{*}{$\begin{array}{l}\text { HEIS } \\
\text { Number }\end{array}$} & \multirow{2}{*}{$\begin{array}{l}\text { Sample } \\
\text { Date }\end{array}$} & \multicolumn{3}{|c|}{ Silver } & \multicolumn{3}{|c|}{ Sodium } & \multicolumn{3}{|c|}{ Vanadium } & \multicolumn{3}{|c|}{ Zinc } & \multicolumn{3}{|c|}{$\begin{array}{c}\text { Total petroleum } \\
\text { hydrocarbons }\end{array}$} & \multicolumn{3}{|c|}{ Bromide } & \multicolumn{3}{|c|}{ Chloride } \\
\hline & & & $\mathrm{mg} / \mathrm{kg}$ & $Q$ & $\mathrm{PQL}$ & $\mathrm{mg} / \mathrm{kg}$ & $Q$ & $\mathrm{PQL}$ & $\mathrm{mg} / \mathrm{kg}$ & $Q$ & PQL & $\mathrm{mg} / \mathrm{kg}$ & Q & $\mathrm{PQL}$ & $\mathrm{mg} / \mathrm{kg}$ & $Q$ & PQL & $\mathrm{mg} / \mathrm{kg}$ & $Q$ & $\mathrm{PQL}$ & $\mathrm{mg} / \mathrm{kg}$ & $Q$ & $\mathrm{PQL}$ \\
\hline NW-1 & $\mathrm{J} 16343$ & $12 / 3 / 2007$ & 0.28 & $\mathrm{U}$ & 0.28 & 204 & C & 5.6 & 50.1 & & 0.39 & 36.6 & C & 1.7 & 139 & $\mathrm{U}$ & 139 & 2.3 & $\mathrm{U}$ & 2.3 & 2.3 & $\mathrm{U}$ & 2.3 \\
\hline NW-2 & $\mathrm{J} 16344$ & $12 / 3 / 2007$ & 0.31 & $\mathrm{U}$ & 0.31 & 190 & $\mathrm{C}$ & 6.2 & 45.7 & & 0.43 & 38.7 & $\mathrm{C}$ & 1.9 & 148 & $\mathrm{U}$ & 148 & 2.6 & $\mathrm{U}$ & 2.6 & 2.6 & $\mathrm{U}$ & 2.6 \\
\hline NW-3 & $\mathrm{J} 16345$ & $12 / 3 / 2007$ & 0.29 & $\mathrm{U}$ & 0.29 & 156 & $\mathrm{C}$ & 5.8 & 41.9 & & 0.4 & 34 & \begin{tabular}{|l|} 
C \\
\end{tabular} & 1.7 & 146 & $\mathrm{U}$ & 146 & 2.4 & $\mathrm{U}$ & 2.4 & 2.4 & $\mathrm{U}$ & 2.4 \\
\hline NW-4 & $\mathrm{J} 16346$ & $12 / 3 / 2007$ & 0.31 & $\mathrm{U}$ & 0.31 & 163 & $C$ & 6.3 & 41.4 & & 0.44 & 44.6 & C & 1.9 & 148 & $\mathrm{U}$ & 148 & 2.3 & $\mathrm{U}$ & 2.3 & 2.3 & $\mathrm{U}$ & 2.3 \\
\hline NW-5 & $\mathrm{J} 16347$ & $12 / 3 / 2007$ & 0.38 & & 0.32 & 153 & $\mathrm{C}$ & 6.4 & 41.8 & & 0.45 & 44.1 & \begin{tabular}{|l|}
$\mathrm{C}$ \\
\end{tabular} & 1.9 & 148 & $\mathrm{U}$ & 148 & 2.4 & $\mathrm{U}$ & 2.4 & 2.4 & $\mathrm{U}$ & 2.4 \\
\hline NW-6 & $\mathrm{J} 16348$ & $12 / 3 / 2007$ & 0.72 & & 0.29 & 179 & $\mathrm{C}$ & 5.9 & 44.6 & & 0.41 & 43.7 & C & 1.8 & 148 & $\mathrm{U}$ & 148 & 2.6 & $\mathrm{U}$ & 2.6 & 2.6 & $\mathrm{U}$ & 2.6 \\
\hline NW-7 & J16349 & $12 / 3 / 2007$ & 0.46 & & 0.29 & 129 & $\mathrm{C}$ & 5.9 & 32.8 & & 0.41 & 38.9 & C & 1.8 & 147 & $\mathrm{U}$ & 147 & 2.6 & $\mathrm{U}$ & 2.6 & 2.6 & $\mathrm{U}$ & 2.6 \\
\hline $\begin{array}{l}\text { Dup of } \\
\text { J16349 }\end{array}$ & $\mathrm{J} 16350$ & $12 / 3 / 2007$ & 0.31 & $\mathrm{U}$ & 0.31 & 147 & $\mathrm{C}$ & 6.1 & 39.9 & & 0.43 & 37.8 & C & 1.8 & 147 & U & 147 & 2.4 & $\mathrm{U}$ & 2.4 & 2.4 & $\mathrm{U}$ & 2.4 \\
\hline NW-8 & $\mathrm{J} 16351$ & $12 / 3 / 2007$ & 0.31 & $\mathrm{U}$ & 0.31 & 135 & $\mathrm{C}$ & 6.2 & 34 & & 0.43 & 43.9 & $\begin{array}{ll}\text { C } \\
\end{array}$ & 1.9 & 140 & $\mathrm{U}$ & 140 & 2.3 & $\mathrm{U}$ & 2.3 & 2.3 & $\mathrm{U}$ & 2.3 \\
\hline NW-9 & $\mathrm{J} 16352$ & $12 / 3 / 2007$ & 0.31 & $\mathrm{U}$ & 0.31 & 123 & $C$ & 6.1 & 30.9 & & 0.43 & 34.5 & C & 1.8 & 150 & $\mathrm{U}$ & 150 & 2.7 & $\mathrm{U}$ & 2.7 & 2.7 & $\mathrm{U}$ & 2.7 \\
\hline NW-10 & $\mathrm{J} 16353$ & $12 / 3 / 2007$ & 0.31 & $\mathrm{U}$ & 0.31 & 154 & $\mathrm{C}$ & 6.1 & 40.9 & & 0.43 & 32.3 & $\mathrm{C}$ & 1.8 & 149 & $\mathrm{U}$ & 149 & 2.2 & $\mathrm{U}$ & 2.2 & 2.2 & $\mathrm{U}$ & 2.2 \\
\hline
\end{tabular}

\begin{tabular}{|c|c|c|c|c|c|c|c|c|c|c|c|c|c|c|c|c|c|c|c|c|c|c|c|}
\hline \multirow{2}{*}{$\begin{array}{l}\text { Sample } \\
\text { Location }\end{array}$} & \multirow{2}{*}{$\begin{array}{c}\text { HEIS } \\
\text { Number }\end{array}$} & \multirow{2}{*}{$\begin{array}{c}\text { Sample } \\
\text { Date }\end{array}$} & \multicolumn{3}{|c|}{ Cyanide } & \multicolumn{3}{|c|}{ Fluoride } & \multicolumn{3}{|c|}{ Nitrate } & \multicolumn{3}{|c|}{ Nitrate } & \multicolumn{3}{|c|}{ Phosphate } & \multicolumn{3}{|c|}{ Sulfate } & \multicolumn{3}{|c|}{ Sulfide } \\
\hline & & & $\mathrm{mg} / \mathrm{kg}$ & $Q$ & PQL & $\mathrm{mg} / \mathrm{kg}$ & $Q$ & PQL & $\mathrm{mg} / \mathrm{kg}$ & $Q$ & $\overline{\mathrm{PQL}}$ & $\mathrm{mg} / \mathrm{kg}$ & Q & $\overline{P Q L}$ & $\mathrm{mg} / \mathrm{kg}$ & Q & PQL & $\mathrm{mg} / \mathrm{kg}$ & Q & PQL & $\mathrm{mg} / \mathrm{kg}$ & \begin{tabular}{l|l} 
Q \\
\end{tabular} & $\overline{\mathrm{PQL}}$ \\
\hline NW-1 & $\mathrm{J} 16343$ & $12 / 3 / 2007$ & 0.49 & $\mathrm{U}$ & 0.49 & 2.3 & $\overrightarrow{\mathrm{U}}$ & 2.3 & 2.26 & $\overrightarrow{\mathrm{U}}$ & 2.3 & 2.26 & $\mathrm{U}$ & 2.3 & 2.3 & $\overrightarrow{\mathrm{U}}$ & 2.3 & 2.3 & $\mathrm{U}$ & 2.3 & 0.22 & $\overrightarrow{\mathrm{U}}$ & 0.22 \\
\hline NW-2 & $\mathrm{J} 16344$ & $12 / 3 / 2007$ & 0.56 & $\mathrm{U}$ & 0.56 & 2.6 & $\mathrm{U}$ & 2.6 & 4.94 & & 2.6 & 2.58 & $\mathrm{U}$ & 2.6 & 2.6 & $\mathrm{U}$ & 2.6 & 104 & & 2.6 & 27.6 & $\mathrm{U}$ & 27.6 \\
\hline NW-3 & $\mathrm{J} 16345$ & $12 / 3 / 2007$ & 0.54 & $\bar{U}$ & 0.54 & 2.4 & $\mathrm{U}$ & 2.4 & 3.05 & & 2.4 & 2.43 & $\mathrm{U}$ & 2.4 & 2.4 & $\mathrm{U}$ & 2.4 & 10.1 & & 2.4 & 24 & U & 24 \\
\hline NW-4 & $\mathrm{J} 16346$ & $12 / 3 / 2007$ & 0.55 & $\mathrm{U}$ & 0.55 & 2.3 & $\bar{U}$ & 2.3 & 2.33 & $\mathrm{U}$ & 2.3 & 2.33 & $\mathrm{U}$ & 2.3 & 2.3 & $\mathrm{U}$ & 2.3 & 2.3 & $\mathrm{U}$ & 2.3 & 29.6 & $\mathrm{U}$ & 29.6 \\
\hline NW-5 & $J 16347$ & $12 / 3 / 2007$ & 0.55 & U & 0.55 & 2.4 & $\mathrm{U}$ & 2.4 & 2.45 & $\mathrm{U}$ & 2.4 & 2.45 & $\mathrm{U}$ & 2.4 & 2.4 & $\mathrm{U}$ & 2.4 & 2.4 & $\mathrm{U}$ & 2.4 & 27.3 & $\mathrm{U}$ & 27.3 \\
\hline NW-6 & $\mathrm{J} 16348$ & $12 / 3 / 2007$ & 0.55 & $\mathrm{U}$ & 0.55 & 2.6 & $\mathrm{U}$ & 2.6 & 2.56 & $\mathrm{U}$ & 2.6 & 2.56 & $\mathrm{U}$ & 2.6 & 3.3 & & 2.6 & 2.6 & $\mathrm{U}$ & 2.6 & 21.8 & $\mathrm{U}$ & 21.8 \\
\hline NW-7 & $\mathrm{J} 16349$ & $12 / 3 / 2007$ & 0.53 & $\mathrm{U}$ & 0.53 & 2.6 & $\mathrm{U}$ & 2.6 & 2.55 & $\mathrm{U}$ & 2.6 & 2.55 & $\mathrm{U}$ & 2.6 & 2.6 & $\mathrm{U}$ & 2.6 & 2.6 & $\mathrm{U}$ & 2.6 & 22.5 & $\mathrm{U}$ & 22.5 \\
\hline Dup of & & & & & & & & & & & & & & & & & & & & & & & \\
\hline $\mathrm{J} 16349$ & $\mathrm{~J} 16350$ & $12 / 3 / 2007$ & 0.48 & U & 0.48 & 2.4 & U & 2.4 & 2.38 & $\mathrm{U}$ & 2.4 & 2.38 & $\mathrm{U}$ & 2.4 & 2.7 & & 2.4 & 2.4 & $\mathrm{U}$ & 2.4 & 22.8 & $\mathrm{U}$ & 22.8 \\
\hline NW-8 & $\mathrm{J} 16351$ & $12 / 3 / 2007$ & 0.45 & $\mathrm{U}$ & 0.45 & 2.3 & $\mathrm{U}$ & 2.3 & 2.3 & $\mathrm{U}$ & 2.3 & 2.3 & $\mathrm{U}$ & 2.3 & 2.3 & $\mathrm{U}$ & 2.3 & 2.3 & $\mathrm{U}$ & 2.3 & 23.2 & $\mathrm{U}$ & 23.2 \\
\hline NW-9 & $\mathrm{J} 16352$ & $12 / 3 / 2007$ & 0.49 & $\mathrm{U}$ & 0.49 & 2.7 & $\mathrm{U}$ & 2.7 & 2.68 & $\mathrm{U}$ & 2.7 & 2.68 & $\mathrm{U}$ & 2.7 & 2.7 & $\mathrm{U}$ & 2.7 & 2.7 & $\mathrm{U}$ & 2.7 & 23.4 & $\mathrm{U}$ & 23.4 \\
\hline NW-10 & $J 16353$ & $12 / 3 / 2007$ & 0.56 & $\mathrm{U}$ & 0.56 & 2.2 & $\mathrm{U}$ & 2.2 & 2.93 & & 2.2 & 2.24 & $\mathrm{U}$ & 2.2 & 4.9 & & 2.2 & 3.1 & & 2.2 & 24.5 & $\mathrm{U}$ & 24.5 \\
\hline
\end{tabular}


Table A-2. 120-F-1 Northwest Excavation Sampling Results from Initial Verification Samples. (8 Pages)

\begin{tabular}{|c|c|c|c|c|c|c|c|c|c|c|c|c|}
\hline \multirow[t]{2}{*}{ Constituent } & \multicolumn{3}{|c|}{$\begin{array}{c}\mathrm{J16343} \\
\text { Sample Location NW-1 } \\
\text { Sample Date 12/3/08 }\end{array}$} & \multicolumn{3}{|c|}{$\begin{array}{c}\text { J16344 } \\
\text { Sample Location NW-2 } \\
\text { Sample Date 12/3/08 }\end{array}$} & \multicolumn{3}{|c|}{$\begin{array}{c}\mathbf{J 1 6 3 4 5} \\
\text { Sample Location NW-3 } \\
\text { Sample Date } 12 / 3 / 08\end{array}$} & \multicolumn{3}{|c|}{$\begin{array}{c}\text { J16346 } \\
\text { Sample Location NW-4 } \\
\text { Sample Date 12/3/08 }\end{array}$} \\
\hline & $\mu \mathrm{g} / \mathrm{kg}$ & $Q$ & PQL & $\mu \mathrm{g} / \mathrm{kg}$ & $Q$ & $\mathbf{P Q L}$ & $\mu \mathrm{g} / \mathrm{kg}$ & $Q$ & $\mathbf{P Q L}$ & $\mu \mathrm{g} / \mathrm{kg}$ & $Q$ & $\mathbf{P Q L}$ \\
\hline \multicolumn{13}{|c|}{ Polychlorinated Biphenyls } \\
\hline Aroclor-1016 & 14 & $\mathrm{U}$ & 14 & 15 & $\mathrm{U}$ & 15 & 15 & $\mathrm{U}$ & 15 & 15 & $\bar{U}$ & 15 \\
\hline Aroclor-1221 & 14 & $\mathrm{U}$ & 14 & 15 & $\mathrm{U}$ & 15 & 15 & $\mathrm{U}$ & 15 & 15 & $\mathrm{U}$ & 15 \\
\hline Aroclor-1232 & 14 & $\mathrm{U}$ & 14 & 15 & $\mathrm{U}$ & 15 & 15 & $\mathrm{U}$ & 15 & 15 & $\mathrm{U}$ & 15 \\
\hline Aroclor-1242 & 14 & $\mathrm{U}$ & 14 & 15 & $\mathrm{U}$ & 15 & 15 & $\mathrm{U}$ & 15 & 15 & $\mathrm{U}$ & 15 \\
\hline Aroclor-1248 & 14 & $\mathrm{U}$ & 14 & 15 & $\mathrm{U}$ & 15 & 15 & $\mathrm{U}$ & 15 & 15 & $\mathrm{U}$ & 15 \\
\hline Aroclor-1254 & 14 & $\mathrm{U}$ & 14 & 15 & $\mathrm{U}$ & 15 & 15 & $\mathrm{U}$ & 15 & 15 & $U$ & 15 \\
\hline Aroclor-1260 & 14 & $\mathrm{U}$ & 14 & 15 & $\mathrm{U}$ & 15 & 15 & $\mathrm{U}$ & 15 & 15 & $\mathrm{U}$ & 15 \\
\hline \multicolumn{13}{|c|}{ Pesticides } \\
\hline Aldrin & 1.4 & UD & 1.4 & 1.5 & UD & 1.5 & 1.5 & UD & 1.5 & 1.5 & UD & 1.5 \\
\hline Alpha-BHC & 1.4 & UD & 1.4 & 1.5 & UD & 1.5 & 1.5 & UD & 1.5 & 1.5 & UD & 1.5 \\
\hline \begin{tabular}{|l} 
Alpha-Chlordane \\
\end{tabular} & 1.8 & \begin{tabular}{|l|} 
JD \\
\end{tabular} & 1.4 & 1.5 & UD & 1.5 & 9.7 & $\mathrm{D}$ & 1.5 & 29 & \begin{tabular}{|l|}
$\mathrm{D}$ \\
\end{tabular} & 1.5 \\
\hline Beta-BHC & 1.4 & UD & 1.4 & 1.5 & UD & 1.5 & 1.5 & UD & 1.5 & 1.5 & UD & 1.5 \\
\hline Delta-BHC & 1.4 & UD & 1.4 & 1.5 & UD & 1.5 & 1.5 & UD & 1.5 & 1.5 & UD & 1.5 \\
\hline \begin{tabular}{|l|} 
Dichlorodiphenyldichloroethane \\
\end{tabular} & 1.4 & UD & 1.4 & 1.5 & UD & 1.5 & 1.5 & UD & 1.5 & 1.5 & UD & 1.5 \\
\hline \begin{tabular}{|l|} 
Dichlorodiphenyldichloroethylene \\
\end{tabular} & 1.4 & UD & 1.4 & 1.5 & UD & 1.5 & 1.5 & UD & 1.5 & 1.5 & UD & 1.5 \\
\hline \begin{tabular}{|l|} 
Dichlorodiphenyltrichloroethane \\
\end{tabular} & 1.4 & UD & 1.4 & 1.5 & UD & 1.5 & 1.5 & UD & 1.5 & 1.5 & UD & 1.5 \\
\hline \begin{tabular}{|l|} 
Dieldrin \\
\end{tabular} & 1.4 & UD & 1.4 & 1.5 & UD & 1.5 & 1.5 & UD & 1.5 & 1.5 & UD & 1.5 \\
\hline \begin{tabular}{|l|} 
Endosulfan I \\
\end{tabular} & 1.4 & UD & 1.4 & 1.5 & UD & 1.5 & 1.5 & UD & 1.5 & 1.5 & UD & 1.5 \\
\hline Endosulfan II & 1.4 & UD & 1.4 & 1.5 & UD & 1.5 & 1.5 & UD & 1.5 & 1.5 & UD & 1.5 \\
\hline \begin{tabular}{|l} 
Endosulfan sulfate \\
\end{tabular} & 1.4 & UD & 1.4 & 1.5 & UD & 1.5 & 1.5 & UD & 1.5 & 1.5 & UD & 1.5 \\
\hline Endrin & 1.4 & UD & 1.4 & 1.5 & UD & 1.5 & 1.5 & UD & 1.5 & 1.6 & \begin{tabular}{|l|l} 
JD \\
\end{tabular} & 1.6 \\
\hline \begin{tabular}{|l} 
Endrin aldehyde \\
\end{tabular} & 1.4 & UD & 1.4 & 1.5 & UD & 1.5 & 1.5 & UD & 1.5 & 1.5 & UD & 1.5 \\
\hline \begin{tabular}{|l} 
Endrin ketone \\
\end{tabular} & 1.4 & UD & 1.4 & 1.5 & UD & 1.5 & 1.5 & UD & 1.5 & 1.5 & UD & 1.5 \\
\hline \begin{tabular}{|l} 
Gamma-BHC (Lindane) \\
\end{tabular} & 1.4 & UD & 1.4 & 1.5 & UD & 1.5 & 1.5 & UD & 1.5 & 1.5 & UD & 1.5 \\
\hline \begin{tabular}{|l} 
gamma-Chlordane \\
\end{tabular} & 2 & \begin{tabular}{|l|l}
$\mathrm{JD}$ \\
\end{tabular} & 1.4 & 1.5 & UD & 1.5 & 9.5 & $\mathrm{D}$ & 1.5 & 32 & \begin{tabular}{|l|l}
$\mathrm{D}$ \\
\end{tabular} & 1.5 \\
\hline Heptachlor & 1.4 & \begin{tabular}{|l|} 
UD \\
\end{tabular} & 1.4 & 1.5 & UD & 1.5 & 1.5 & UD & 1.5 & 11 & \begin{tabular}{|l|}
$\mathrm{D}$ \\
\end{tabular} & 11 \\
\hline Heptachlor epoxide & 1.4 & UD & 1.4 & 1.5 & UD & 1.5 & 1.5 & UD & 1.5 & 1.5 & UD & 1.5 \\
\hline Methoxychlor & 1.4 & UD & 1.4 & 1.5 & UD & 1.5 & 1.5 & UD & 1.5 & 1.5 & UD & 1.5 \\
\hline Toxaphene & 14 & UD & 14 & 15 & UD & 15 & 15 & UD & 15 & 15 & UD & 15 \\
\hline \multicolumn{13}{|c|}{ Semivolatile Organic Analytes } \\
\hline 1,2,4-Trichlorobenzene & 350 & U & 350 & 370 & U & 370 & 370 & $\mathrm{U}$ & 370 & 370 & $\mathrm{U}$ & 370 \\
\hline 1,2-Dichlorobenzene & 350 & $\mathrm{U}$ & 350 & 370 & U & 370 & 370 & $\mathrm{U}$ & 370 & 370 & $\mathrm{U}$ & 370 \\
\hline 1,3-Dichlorobenzene & 350 & $\mathrm{U}$ & 350 & 370 & U & 370 & 370 & $\mathrm{U}$ & 370 & 370 & U & 370 \\
\hline \begin{tabular}{|l|}
$1,4-D i c h l o r o b e n z e n e$ \\
\end{tabular} & 350 & $\mathrm{U}$ & 350 & 370 & U & 370 & 370 & $\mathrm{U}$ & 370 & 370 & \begin{tabular}{|l}
$\mathrm{U}$ \\
\end{tabular} & 370 \\
\hline 2,4,5-Trichlorophenol & 870 & $\mathrm{U}$ & 870 & 940 & U & 940 & 870 & $\mathrm{U}$ & 870 & 930 & $\mathrm{U}$ & 930 \\
\hline 2,4,6-Trichlorophenol & 350 & $\mathrm{U}$ & 350 & 370 & $\mathrm{U}$ & 370 & 370 & $\mathrm{U}$ & 370 & 370 & U & 370 \\
\hline 2,4-Dichlorophenol & 350 & $\mathrm{U}$ & 350 & 370 & $\mathrm{U}$ & 370 & 370 & $\mathrm{U}$ & 370 & 370 & $\mathrm{U}$ & 370 \\
\hline 2,4-Dimethylphenol & 350 & $\mathrm{U}$ & 350 & 370 & $\mathrm{U}$ & 370 & 370 & $\mathrm{U}$ & 370 & 370 & \begin{tabular}{|l|}
$U$ \\
\end{tabular} & 370 \\
\hline 2,4-Dinitrophenol & 870 & $\mathrm{U}$ & 870 & 940 & U & 940 & 910 & $\mathrm{U}$ & 910 & 930 & $\mathrm{U}$ & 930 \\
\hline 2,4-Dinitrotoluene & 350 & $\mathrm{U}$ & 350 & 370 & U & 370 & 370 & $\mathrm{U}$ & 370 & 370 & U & 370 \\
\hline 2,6-Dinitrotoluene & 350 & $\mathrm{U}$ & 350 & 370 & $\mathrm{U}$ & 370 & 370 & $\mathrm{U}$ & 370 & 370 & \begin{tabular}{|l|}
$\mathrm{U}$ \\
\end{tabular} & 370 \\
\hline 2-Chloronaphthalene & 350 & $\mathrm{U}$ & 350 & 370 & $\mathrm{U}$ & 370 & 370 & $\mathrm{U}$ & 370 & 370 & U & 370 \\
\hline \begin{tabular}{|l|} 
2-Chlorophenol \\
\end{tabular} & 350 & $\mathrm{U}$ & 350 & 370 & $\mathrm{U}$ & 370 & 370 & $\mathrm{U}$ & 370 & 370 & \begin{tabular}{|l|}
$\mathrm{U}$ \\
\end{tabular} & 370 \\
\hline 2-Methylnaphthalene & 350 & $\mathrm{U}$ & 350 & 370 & U & 370 & 370 & $\mathrm{U}$ & 370 & 370 & $\mathrm{U}$ & 370 \\
\hline 2-Methylphenol (cresol, o-) & 350 & $\mathrm{U}$ & 350 & 370 & $\mathrm{U}$ & 370 & 370 & $\mathrm{U}$ & 370 & 370 & $\mathrm{U}$ & 370 \\
\hline 2-Nitroaniline & 870 & $\mathrm{U}$ & 870 & 940 & $\mathrm{U}$ & 940 & 910 & $\mathrm{U}$ & 910 & 930 & $\mathrm{U}$ & 930 \\
\hline 2-Nitrophenol & 350 & $\mathrm{U}$ & 350 & 370 & $\mathrm{U}$ & 370 & 370 & $\mathrm{U}$ & 370 & 370 & $U$ & 370 \\
\hline
\end{tabular}


Table A-2. 120-F-1 Northwest Excavation Sampling Results from Initial Verification Samples. (8 Pages)

\begin{tabular}{|c|c|c|c|c|c|c|c|c|c|c|c|c|}
\hline \multirow[t]{2}{*}{ Constituent } & \multicolumn{3}{|c|}{$\begin{array}{c}\text { J16343 } \\
\text { Sample Location NW-1 } \\
\text { Sample Date 12/3/08 }\end{array}$} & \multicolumn{3}{|c|}{$\begin{array}{c}\text { J16344 } \\
\text { Sample Location NW-2 } \\
\text { Sample Date 12/3/08 }\end{array}$} & \multicolumn{3}{|c|}{$\begin{array}{c}\text { J16345 } \\
\text { Sample Location NW-3 } \\
\text { Sample Date } 12 / 3 / 08\end{array}$} & \multicolumn{3}{|c|}{$\begin{array}{c}\text { J16346 } \\
\text { Sample Location NW-4 } \\
\text { Sample Date } 12 / 3 / 08\end{array}$} \\
\hline & $\mu \mathrm{g} / \mathrm{kg}$ & $Q$ & PQL & $\mu \mathrm{g} / \mathrm{kg}$ & $Q$ & PQL & $\mu \mathrm{g} / \mathrm{kg}$ & $Q$ & PQL & $\mu \mathrm{g} / \mathrm{kg}$ & $Q$ & PQL \\
\hline \multicolumn{13}{|c|}{ Semivolatile Organic Analytes (continued) } \\
\hline 3,3'-Dichlorobenzidine & 350 & $\mathrm{U}$ & 350 & 370 & $\mathrm{U}$ & 370 & 370 & $\mathrm{U}$ & 370 & 370 & $\mathrm{U}$ & 370 \\
\hline 4-Methylphenol (p-cresol) & 350 & $\mathrm{U}$ & 350 & 370 & $\mathrm{U}$ & 370 & 370 & $\mathrm{U}$ & 370 & 370 & $\mathrm{U}$ & 370 \\
\hline 3-Nitroaniline & 870 & $\mathrm{U}$ & 870 & 940 & $\mathrm{U}$ & 940 & 910 & $\mathrm{U}$ & 910 & 930 & $\mathrm{U}$ & 930 \\
\hline 4,6-Dinitro-2-methylphenol & 870 & $\mathrm{U}$ & 870 & 940 & $\mathrm{U}$ & 940 & 910 & $\mathrm{U}$ & 910 & 930 & $\mathrm{U}$ & 930 \\
\hline 4-Bromophenyl-phenylether & 350 & $\mathrm{U}$ & 350 & 370 & $\mathrm{U}$ & 370 & 370 & $\mathrm{U}$ & 370 & 370 & $\mathrm{U}$ & 370 \\
\hline 4-Chloro-3-methylphenol & 350 & $\mathrm{U}$ & 350 & 370 & $\mathrm{U}$ & 370 & 370 & $\mathrm{U}$ & 370 & 370 & $\mathrm{U}$ & 370 \\
\hline 4-Chloroaniline & 350 & $\mathrm{U}$ & 350 & 370 & $\mathrm{U}$ & 370 & 370 & $\mathrm{U}$ & 370 & 370 & $\mathrm{U}$ & 370 \\
\hline 4-Chlorophenyl-phenylether & 350 & $\mathrm{U}$ & 350 & 370 & $\mathrm{U}$ & 370 & 370 & $\mathrm{U}$ & 370 & 370 & $\mathrm{U}$ & 370 \\
\hline 4-Nitroaniline & 870 & $\mathrm{U}$ & 870 & 940 & $\mathrm{U}$ & 940 & 910 & $\mathrm{U}$ & 910 & 930 & $\mathrm{U}$ & 930 \\
\hline 4-Nitrophenol & 870 & $\mathrm{U}$ & 870 & 940 & $\mathrm{U}$ & 940 & 910 & $\mathrm{U}$ & 910 & 930 & $\mathrm{U}$ & 930 \\
\hline Acenaphthene & 350 & $\mathrm{U}$ & 350 & 370 & $\mathrm{U}$ & 370 & 370 & $\mathrm{U}$ & 370 & 370 & $\mathrm{U}$ & 370 \\
\hline Acenaphthylene & 350 & $\mathrm{U}$ & 350 & 370 & $\mathrm{U}$ & 370 & 370 & $\mathrm{U}$ & 370 & 370 & $\mathrm{U}$ & 370 \\
\hline Anthracene & 350 & $\mathrm{U}$ & 350 & 370 & $\mathrm{U}$ & 370 & 370 & $\mathrm{U}$ & 370 & 370 & $\mathrm{U}$ & 370 \\
\hline Benzo(a)anthracene & 350 & $\mathrm{U}$ & 350 & 370 & $\mathrm{U}$ & 370 & 370 & $\mathrm{U}$ & 370 & 370 & $\mathrm{U}$ & 370 \\
\hline Benzo(a)pyrene & 350 & $\mathrm{U}$ & 350 & 370 & $\mathrm{U}$ & 370 & 370 & $\mathrm{U}$ & 370 & 370 & $\mathrm{U}$ & 370 \\
\hline Benzo(b)fluoranthene & 350 & $\mathrm{U}$ & 350 & 370 & $\mathrm{U}$ & 370 & 370 & $\mathrm{U}$ & 370 & 370 & $\mathrm{U}$ & 370 \\
\hline Benzo(g,h,i)perylene & 350 & $\mathrm{U}$ & 350 & 370 & $\mathrm{U}$ & 370 & 370 & $\mathrm{U}$ & 370 & 370 & $\mathrm{U}$ & 370 \\
\hline Benzo(k)fluoranthene & 350 & $\mathrm{U}$ & 350 & 370 & $\mathrm{U}$ & 370 & 370 & $\mathrm{U}$ & 370 & 370 & $\mathrm{U}$ & 370 \\
\hline Bis(2-chloro-1-methylethyl)ether & 350 & $\mathrm{U}$ & 350 & 370 & $\mathrm{U}$ & 370 & 370 & $\mathrm{U}$ & 370 & 370 & $\mathrm{U}$ & 370 \\
\hline Bis(2-chloroethoxy)methane & 350 & $\mathrm{U}$ & 350 & 370 & $\mathrm{U}$ & 370 & 370 & $\mathrm{U}$ & 370 & 370 & $\mathrm{U}$ & 370 \\
\hline Bis(2-chloroethyl) ether & 350 & $\mathrm{U}$ & 350 & 370 & $\mathrm{U}$ & 370 & 370 & $\mathrm{U}$ & 370 & 370 & $\mathrm{U}$ & 370 \\
\hline Bis(2-ethylhexyl) phthalate & 23 & $\mathrm{JB}$ & 350 & 34 & $\mathrm{JB}$ & 370 & 41 & $\mathrm{JB}$ & 370 & 56 & $\mathrm{JB}$ & 370 \\
\hline Butylbenzylphthalate & 350 & $\mathrm{U}$ & 350 & 370 & $\mathrm{U}$ & 370 & 370 & $\mathrm{U}$ & 370 & 370 & $\mathrm{U}$ & 370 \\
\hline Carbazole & 350 & $\mathrm{U}$ & 350 & 370 & $\mathrm{U}$ & 370 & 370 & $\mathrm{U}$ & 370 & 370 & $\mathrm{U}$ & 370 \\
\hline Chrysene & 350 & $\mathrm{U}$ & 350 & 370 & $\mathrm{U}$ & 370 & 370 & $\mathrm{U}$ & 370 & 370 & $\mathrm{U}$ & 370 \\
\hline Dibenz $(\mathrm{a}, \mathrm{h})$ anthracene & 350 & $\mathrm{U}$ & 350 & 370 & $\mathrm{U}$ & 370 & 370 & $\mathrm{U}$ & 370 & 370 & $\mathrm{U}$ & 370 \\
\hline Dibenzofuran & 350 & $\mathrm{U}$ & 350 & 370 & $\mathrm{U}$ & 370 & 370 & $\mathrm{U}$ & 370 & 370 & $\mathrm{U}$ & 370 \\
\hline Diethylphthalate & 350 & $\mathrm{U}$ & 350 & 370 & $\mathrm{U}$ & 370 & 370 & $\mathrm{U}$ & 370 & 370 & $\mathrm{U}$ & 370 \\
\hline Dimethylphthalate & 350 & $\mathrm{U}$ & 350 & 370 & $\mathrm{U}$ & 370 & 370 & $\mathrm{U}$ & 370 & 370 & $\mathrm{U}$ & 370 \\
\hline Di-n-butylphthalate & 350 & $\mathrm{U}$ & 350 & 370 & $\mathrm{U}$ & 370 & 370 & $\mathrm{U}$ & 370 & 370 & $\mathrm{U}$ & 370 \\
\hline Di-n-octylphthalate & 350 & $\mathrm{U}$ & 350 & 370 & $\mathrm{U}$ & 370 & 370 & $\mathrm{U}$ & 370 & 370 & $\mathrm{U}$ & 370 \\
\hline Fluoranthene & 350 & $\mathrm{U}$ & 350 & 370 & $\mathrm{U}$ & 370 & 370 & $\mathrm{U}$ & 370 & 370 & $\mathrm{U}$ & 370 \\
\hline Fluorene & 350 & $\mathrm{U}$ & 350 & 370 & $\mathrm{U}$ & 370 & 370 & $\mathrm{U}$ & 370 & 370 & $\mathrm{U}$ & 370 \\
\hline Hexachlorobenzene & 350 & $\mathrm{U}$ & 350 & 370 & $\mathrm{U}$ & 370 & 370 & $\mathrm{U}$ & 370 & 370 & $\mathrm{U}$ & 370 \\
\hline Hexachlorobutadiene & 350 & $\mathrm{U}$ & 350 & 370 & $\mathrm{U}$ & 370 & 370 & $\mathrm{U}$ & 370 & 370 & $\mathrm{U}$ & 370 \\
\hline Hexachlorocyclopentadiene & 350 & $\mathrm{U}$ & 350 & 370 & $\mathrm{U}$ & 370 & 370 & $\mathrm{U}$ & 370 & 370 & $\mathrm{U}$ & 370 \\
\hline Hexachloroethane & 350 & $\mathrm{U}$ & 350 & 370 & $\mathrm{U}$ & 370 & 370 & $\mathrm{U}$ & 370 & 370 & $\mathrm{U}$ & 370 \\
\hline Indeno(1,2,3-cd)pyrene & 350 & $\mathrm{U}$ & 350 & 370 & $\mathrm{U}$ & 370 & 370 & $\mathrm{U}$ & 370 & 370 & $\mathrm{U}$ & 370 \\
\hline Isophorone & 350 & $\mathrm{U}$ & 350 & 370 & $\mathrm{U}$ & 370 & 370 & $\mathrm{U}$ & 370 & 370 & $\mathrm{U}$ & 370 \\
\hline Naphthalene & 350 & $\mathrm{U}$ & 350 & 370 & U & 370 & 370 & $\mathrm{U}$ & 370 & 370 & $\mathrm{U}$ & 370 \\
\hline Nitrobenzene & 350 & $\mathrm{U}$ & 350 & 370 & $\mathrm{U}$ & 370 & 370 & $\mathrm{U}$ & 370 & 370 & $\mathrm{U}$ & 370 \\
\hline N-Nitroso-di-n-dipropylamine & 350 & $\mathrm{U}$ & 350 & 370 & $\mathrm{U}$ & 370 & 370 & $\mathrm{U}$ & 370 & 370 & $\mathrm{U}$ & 370 \\
\hline N-Nitrosodiphenylamine & 350 & $\mathrm{U}$ & 350 & 370 & $\mathrm{U}$ & 370 & 370 & $\mathrm{U}$ & 370 & 370 & $\mathrm{U}$ & 370 \\
\hline Pentachlorophenol & 870 & $\mathrm{U}$ & 870 & 940 & $\mathrm{U}$ & 940 & 910 & $\mathrm{U}$ & 910 & 930 & $\mathrm{U}$ & 930 \\
\hline Phenanthrene & 350 & $\mathrm{U}$ & 350 & 370 & $\mathrm{U}$ & 370 & 370 & $\mathrm{U}$ & 370 & 370 & $\mathrm{U}$ & 370 \\
\hline Phenol & 350 & $\mathrm{U}$ & 350 & 370 & $\mathrm{U}$ & 370 & 370 & $\mathrm{U}$ & 370 & 370 & $\mathrm{U}$ & 370 \\
\hline Pyrene & 350 & $\mathrm{U}$ & 350 & 370 & $\mathrm{U}$ & 370 & 370 & $\mathrm{U}$ & 370 & 370 & $\mathrm{U}$ & 370 \\
\hline
\end{tabular}


Table A-2. 120-F-1 Northwest Excavation Sampling Results from Initial Verification Samples. (8 Pages)

\begin{tabular}{|c|c|c|c|c|c|c|c|c|c|c|c|c|}
\hline \multirow[t]{2}{*}{ Constituent } & \multicolumn{3}{|c|}{$\begin{array}{c}\text { J16347 } \\
\text { Sample Location NW-5 } \\
\text { Sample Date 12/3/08 }\end{array}$} & \multicolumn{3}{|c|}{$\begin{array}{c}\text { J16348 } \\
\text { Sample Location NW-6 } \\
\text { Sample Date 12/3/08 }\end{array}$} & \multicolumn{3}{|c|}{$\begin{array}{c}\text { J16349 } \\
\text { Sample Location NW-7 } \\
\text { Sample Date 12/3/08 }\end{array}$} & \multicolumn{3}{|c|}{$\begin{array}{c}\mathbf{J 1 6 3 5 0} \\
\text { Dup of } \mathbf{J 1 6 3 4 9} \\
\text { Sample Date 12/3/08 }\end{array}$} \\
\hline & $\mu \mathrm{g} / \mathrm{kg}$ & $\mathbf{Q}$ & $\mathrm{PQL}$ & $\mu \mathrm{g} / \mathrm{kg}$ & $Q$ & PQL & $\mu \mathrm{g} / \mathrm{kg}$ & Q & $\mathrm{PQL}$ & $\mu \mathrm{g} / \mathrm{kg}$ & $Q$ & PQL \\
\hline \multicolumn{13}{|c|}{ Polychlorinated Biphenyls } \\
\hline Aroclor-1016 & 15 & $\mathrm{U}$ & 15 & 7400 & UD & 7400 & 7400 & UD & 7400 & 7000 & UD & 7000 \\
\hline Aroclor-1221 & 15 & $\mathrm{U}$ & 15 & 7400 & UD & 7400 & 7400 & UD & 7400 & 7000 & UD & 7000 \\
\hline Aroclor-1232 & 15 & $\mathrm{U}$ & 15 & 7400 & UD & 7400 & 7400 & UD & 7400 & 7000 & UD & 7000 \\
\hline Aroclor-1242 & 15 & $\mathrm{U}$ & 15 & 7400 & UD & 7400 & 7400 & UD & 7400 & 7000 & UD & 7000 \\
\hline Aroclor-1248 & 15 & $\bar{U}$ & 15 & 7400 & UD & 7400 & 7400 & UD & 7400 & 7000 & UD & 7000 \\
\hline Aroclor- 1254 & 15 & $\mathrm{U}$ & 15 & 7400 & UD & 7400 & 7400 & UD & 7400 & 7000 & UD & 7000 \\
\hline Aroclor- 1260 & 15 & $\bar{U}$ & 15 & 7400 & UD & 7400 & 7400 & UD & 7400 & 7000 & UD & 7000 \\
\hline \multicolumn{13}{|c|}{ Pesticides } \\
\hline Aldrin & 3 & UD & 3 & 18 & UD & 18 & 74 & UD & 74 & 70 & UD & 70 \\
\hline Alpha-BHC & 3 & UD & 3 & 18 & UD & 18 & 74 & UD & 74 & 70 & UD & 70 \\
\hline Alpha-Chlordane & 250 & $\mathrm{D}$ & 3 & 2100 & D & 18 & 4900 & $\mathrm{D}$ & 74 & 12000 & D & 70 \\
\hline Beta-BHC & 3 & UD & 3 & 18 & UD & 18 & 74 & UD & 74 & 70 & UD & 70 \\
\hline Delta-BHC & 3 & UD & 3 & 18 & UD & 18 & 74 & UD & 74 & 70 & UD & 70 \\
\hline Dichlorodiphenyldichloroethane & 12 & JXD & 3 & 18 & UD & 18 & 74 & UD & 74 & 530 & $\mathrm{XD}$ & 70 \\
\hline Dichlorodiphenyldichloroethylene & 3 & UD & 3 & 18 & UD & 18 & 74 & UD & 74 & 70 & UD & 70 \\
\hline Dichlorodiphenyltrichloroethane & 3 & UD & 3 & 18 & UD & 18 & 74 & UD & 74 & 70 & UD & 70 \\
\hline Dieldrin & 3 & UD & 3 & 72 & JD & 18 & 180 & $\mathrm{JX}$ & 74 & 440 & $\mathrm{XD}$ & 70 \\
\hline Endosulfan I & 3 & UD & 3 & 18 & UD & 18 & 74 & UD & 74 & 88 & JD & 70 \\
\hline Endosulfan II & 3 & UD & 3 & 18 & UD & 18 & 74 & UD & 74 & 70 & UD & 70 \\
\hline Endosulfan sulfate & 3 & UD & 3 & 18 & UD & 18 & 74 & UD & 74 & 70 & UD & 70 \\
\hline Endrin & 13 & $\mathrm{JD}$ & 3 & 100 & $\mathrm{D}$ & 18 & 240 & JD & 74 & 70 & UD & 70 \\
\hline Endrin aldehyde & 3 & UD & 3 & 18 & UD & 18 & 74 & UD & 74 & 70 & UD & 70 \\
\hline Endrin ketone & 3 & UD & 3 & 18 & UD & 18 & 74 & UD & 74 & 70 & UD & 70 \\
\hline Gamma-BHC (Lindane) & 3 & UD & 3 & 18 & UD & 18 & 74 & UD & 74 & 70 & UD & 70 \\
\hline gamma-Chlordane & 230 & $\mathrm{D}$ & 3 & 2000 & $\mathrm{D}$ & 18 & 4600 & $\mathrm{D}$ & 74 & 12000 & \begin{tabular}{|l|l|}
$\mathrm{D}$ \\
\end{tabular} & 70 \\
\hline Heptachlor & 56 & $\mathrm{D}$ & 3 & 820 & $\mathrm{D}$ & 18 & 2000 & $\mathrm{D}$ & 74 & 5700 & $\mathrm{D}$ & 70 \\
\hline Heptachlor epoxide & 9.1 & JD & 3 & 18 & UD & 18 & 74 & UD & 74 & 70 & UD & 70 \\
\hline Methoxychlor & 3 & UD & 3 & 18 & UD & 18 & 74 & UD & 74 & 70 & UD & 70 \\
\hline Toxaphene & 30 & UD & 30 & 180 & UD & 180 & 740 & UD & 740 & 700 & UD & 700 \\
\hline \multicolumn{13}{|c|}{ Semivolatile Organic Analytes } \\
\hline 1,2,4-Trichlorobenzene & 370 & $\mathrm{U}$ & 370 & 370 & $\mathrm{U}$ & 370 & 370 & $\mathrm{U}$ & 370 & 350 & $\mathrm{U}$ & 350 \\
\hline 1,2-Dichlorobenzene & 370 & $\mathrm{U}$ & 370 & 370 & $\mathrm{U}$ & 370 & 370 & $\mathrm{U}$ & 370 & 350 & $\mathrm{U}$ & 350 \\
\hline 1,3-Dichlorobenzene & 370 & $\mathrm{U}$ & 370 & 370 & $\mathrm{U}$ & 370 & 370 & $\mathrm{U}$ & 370 & 350 & $\mathrm{U}$ & 350 \\
\hline 1,4-Dichlorobenzene & 370 & $\mathrm{U}$ & 370 & 370 & $\mathrm{U}$ & 370 & 370 & $\mathrm{U}$ & 370 & 350 & $\mathrm{U}$ & 350 \\
\hline 2,4,5-Trichlorophenol & 930 & $\mathrm{U}$ & 930 & 920 & $\mathrm{U}$ & 920 & 920 & $\mathrm{U}$ & 920 & 880 & $\mathrm{U}$ & 880 \\
\hline 2,4,6-Trichlorophenol & 370 & $\mathrm{U}$ & 370 & 370 & $\mathrm{U}$ & 370 & 370 & $\mathrm{U}$ & 370 & 350 & $\mathrm{U}$ & 350 \\
\hline 2,4-Dichlorophenol & 370 & $\mathrm{U}$ & 370 & 370 & $\mathrm{U}$ & 370 & 370 & $\mathrm{U}$ & 370 & 350 & $\mathrm{U}$ & 350 \\
\hline 2,4-Dimethylphenol & 370 & $\mathrm{U}$ & 370 & 370 & $\mathrm{U}$ & 370 & 370 & $\mathrm{U}$ & 370 & 350 & $\mathrm{U}$ & 350 \\
\hline 2,4-Dinitrophenol & 930 & $\mathrm{U}$ & 930 & 920 & $U$ & 920 & 920 & $\mathrm{U}$ & 920 & 880 & $\mathrm{U}$ & 880 \\
\hline 2,4-Dinitrotoluene & 370 & $\mathrm{U}$ & 370 & 370 & $\mathrm{U}$ & 370 & 370 & $\mathrm{U}$ & 370 & 350 & $\mathrm{U}$ & 350 \\
\hline 2,6-Dinitrotoluene & 370 & $\mathrm{U}$ & 370 & 370 & $\mathrm{U}$ & 370 & 370 & $\mathrm{U}$ & 370 & 350 & $\mathrm{U}$ & 350 \\
\hline 2-Chloronaphthalene & 370 & $\mathrm{U}$ & 370 & 370 & $\mathrm{U}$ & 370 & 370 & $\mathrm{U}$ & 370 & 350 & $\mathrm{U}$ & 350 \\
\hline 2-Chlorophenol & 370 & $\mathrm{U}$ & 370 & 370 & $\mathrm{U}$ & 370 & 370 & $\mathrm{U}$ & 370 & 350 & $\mathrm{U}$ & 350 \\
\hline 2-Methylnaphthalene & 370 & $\mathrm{U}$ & 370 & 370 & $\mathrm{U}$ & 370 & 370 & $\mathrm{U}$ & 370 & 350 & $\mathrm{U}$ & 350 \\
\hline 2-Methylphenol (cresol, o-) & 370 & $\mathrm{U}$ & 370 & 370 & $\mathrm{U}$ & 370 & 370 & $\mathrm{U}$ & 370 & 350 & $\mathrm{U}$ & 350 \\
\hline 2-Nitroaniline & 930 & $\mathrm{U}$ & 930 & 920 & $\mathrm{U}$ & 920 & 920 & $\mathrm{U}$ & 920 & 880 & $\mathrm{U}$ & 880 \\
\hline 2-Nitrophenol & 370 & $\mathrm{U}$ & 370 & 370 & $\mathrm{U}$ & 370 & 370 & $\mathrm{U}$ & 370 & 350 & $\mathrm{U}$ & 350 \\
\hline
\end{tabular}


Table A-2. 120-F-1 Northwest Excavation Sampling Results from Initial Verification Samples. (8 Pages)

\begin{tabular}{|c|c|c|c|c|c|c|c|c|c|c|c|c|}
\hline \multirow[t]{2}{*}{ Constituent } & \multicolumn{3}{|c|}{\begin{tabular}{|c|}
$\mathbf{J 1 6 3 4 7}$ \\
Sample Location NW-5 \\
Sample Date 12/3/08
\end{tabular}} & \multicolumn{3}{|c|}{$\begin{array}{c}\text { J16348 } \\
\text { Sample Location NW-6 } \\
\text { Sample Date 12/3/08 }\end{array}$} & \multicolumn{3}{|c|}{$\begin{array}{c}\text { J16349 } \\
\text { Sample Location NW-7 } \\
\text { Sample Date 12/3/08 }\end{array}$} & \multicolumn{3}{|c|}{$\begin{array}{c}\mathrm{J16350} \\
\text { Dup of J16349 } \\
\text { Sample Date 12/3/08 }\end{array}$} \\
\hline & $\mu \mathrm{g} / \mathrm{kg}$ & $Q$ & PQL & $\mu \mathrm{g} / \mathrm{kg}$ & $Q$ & PQL & $\mu \mathrm{g} / \mathrm{kg}$ & Q & PQL & $\mu \mathrm{g} / \mathrm{kg}$ & Q & $\mathbf{P Q L}$ \\
\hline \multicolumn{13}{|c|}{ Semivolatile Organic Analytes (continued) } \\
\hline 3,3'-Dichlorobenzidine & 370 & $\mathrm{U}$ & 370 & 370 & $\mathrm{U}$ & 370 & 370 & $\mathrm{U}$ & 370 & 370 & $U$ & 370 \\
\hline 4-Methylphenol (p-cresol) & 370 & $\mathrm{U}$ & 370 & 370 & $\mathrm{U}$ & 370 & 370 & $\mathrm{U}$ & 370 & 370 & $\mathrm{U}$ & 370 \\
\hline 3-Nitroaniline & 930 & $\mathrm{U}$ & 930 & 930 & $\mathrm{U}$ & 930 & 920 & U & 920 & 920 & $\mathrm{U}$ & 920 \\
\hline \begin{tabular}{|l|} 
4,6-Dinitro-2-methylphenol \\
\end{tabular} & 930 & $\mathrm{U}$ & 930 & 930 & $\mathrm{U}$ & 930 & 920 & $\mathrm{U}$ & 920 & 920 & $\mathrm{U}$ & 920 \\
\hline 4-Bromophenyl-phenylether & 370 & $\mathrm{U}$ & 370 & 370 & $\mathrm{U}$ & 370 & 370 & $\mathrm{U}$ & 370 & 370 & $\mathrm{U}$ & 370 \\
\hline 4-Chloro-3-methylphenol & 370 & $\mathrm{U}$ & 370 & 370 & $\mathrm{U}$ & 370 & 370 & $\mathrm{U}$ & 370 & 370 & $\mathrm{U}$ & 370 \\
\hline \begin{tabular}{|l|} 
4-Chloroaniline \\
\end{tabular} & 370 & $\mathrm{U}$ & 370 & 370 & $\mathrm{U}$ & 370 & 370 & U & 370 & 370 & $\mathrm{U}$ & 370 \\
\hline 4-Chlorophenyl-phenylether & 370 & $\mathrm{U}$ & 370 & 370 & $\mathrm{U}$ & 370 & 370 & $\mathrm{U}$ & 370 & 370 & $\mathrm{U}$ & 370 \\
\hline 4-Nitroaniline & 930 & $\mathrm{U}$ & 930 & 930 & $\mathrm{U}$ & 930 & 920 & $\mathrm{U}$ & 920 & 920 & $\mathrm{U}$ & 920 \\
\hline 4-Nitrophenol & 930 & $\mathrm{U}$ & 930 & 930 & $\mathrm{U}$ & 930 & 920 & $\mathrm{U}$ & 920 & 920 & $\mathrm{U}$ & 920 \\
\hline Acenaphthene & 370 & $\mathrm{U}$ & 370 & 370 & $\mathrm{U}$ & 370 & 370 & $\mathrm{U}$ & 370 & 370 & $\mathrm{U}$ & 370 \\
\hline \begin{tabular}{|l} 
Acenaphthylene \\
\end{tabular} & 370 & $\mathrm{U}$ & 370 & 370 & $\mathrm{U}$ & 370 & 370 & $\mathrm{U}$ & 370 & 370 & $\mathrm{U}$ & 370 \\
\hline \begin{tabular}{|l} 
Anthracene \\
\end{tabular} & 370 & $\mathrm{U}$ & 370 & 370 & $\mathrm{U}$ & 370 & 370 & $\mathrm{U}$ & 370 & 370 & $\mathrm{U}$ & 370 \\
\hline Benzo(a)anthracene & 370 & $\mathrm{U}$ & 370 & 370 & $\mathrm{U}$ & 370 & 370 & $\mathrm{U}$ & 370 & 370 & $\mathrm{U}$ & 370 \\
\hline Benzo(a)pyrene & 370 & $\mathrm{U}$ & 370 & 370 & $\mathrm{U}$ & 370 & 370 & $\mathrm{U}$ & 370 & 370 & $\mathrm{U}$ & 370 \\
\hline Benzo(b)fluoranthene & 370 & $\mathrm{U}$ & 370 & 370 & $\mathrm{U}$ & 370 & 370 & $\mathrm{U}$ & 370 & 370 & $\mathrm{U}$ & 370 \\
\hline Benzo $(\mathrm{g}, \mathrm{h}, \mathrm{i})$ perylene & 370 & $\mathrm{U}$ & 370 & 370 & $\mathrm{U}$ & 370 & 370 & $\mathrm{U}$ & 370 & 370 & $\mathrm{U}$ & 370 \\
\hline Benzo(k)fluoranthene & 370 & $\mathrm{U}$ & 370 & 370 & $\mathrm{U}$ & 370 & 370 & $\mathrm{U}$ & 370 & 370 & $\mathrm{u}$ & 370 \\
\hline \begin{tabular}{|l} 
Bis(2-chloro-1-methylethyl)ether \\
\end{tabular} & 370 & $\mathrm{U}$ & 370 & 370 & $\mathrm{U}$ & 370 & 370 & $\mathrm{U}$ & 370 & 370 & $\mathrm{U}$ & 370 \\
\hline \begin{tabular}{|l} 
Bis (2-chloroethoxy)methane \\
\end{tabular} & 370 & $\mathrm{U}$ & 370 & 370 & $\mathrm{U}$ & 370 & 370 & $\mathrm{U}$ & 370 & 370 & $\mathrm{U}$ & 370 \\
\hline Bis(2-chloroethyl) ether & 370 & $\mathrm{U}$ & 370 & 370 & $\mathrm{U}$ & 370 & 370 & $\mathrm{U}$ & 370 & 370 & $\mathrm{U}$ & 370 \\
\hline Bis(2-ethylhexyl) phthalate & 31 & $\mathrm{JB}$ & 370 & 120 & $\mathrm{JB}$ & 370 & 170 & $\mathrm{JB}$ & 370 & 69 & $\mathrm{JB}$ & 370 \\
\hline Butylbenzylphthalate & 370 & $\mathrm{U}$ & 370 & 370 & $\mathrm{U}$ & 370 & 370 & $\mathrm{U}$ & 370 & 370 & $\mathrm{U}$ & 370 \\
\hline \begin{tabular}{|l} 
Carbazole \\
\end{tabular} & 370 & $\mathrm{U}$ & 370 & 370 & $\mathrm{U}$ & 370 & 370 & $\mathrm{U}$ & 370 & 370 & $\mathrm{U}$ & 370 \\
\hline \begin{tabular}{|l} 
Chrysene \\
\end{tabular} & 370 & $\mathrm{U}$ & 370 & 370 & $\mathrm{U}$ & 370 & 370 & $\mathrm{U}$ & 370 & 370 & $\mathrm{U}$ & 370 \\
\hline Dibenz $(\mathrm{a}, \mathrm{h})$ anthracene & 370 & $\mathrm{U}$ & 370 & 75 & $\mathrm{~J}$ & 370 & 110 & $\mathrm{~J}$ & 370 & 370 & $\mathrm{u}$ & 370 \\
\hline \begin{tabular}{|l} 
Dibenzofuran \\
\end{tabular} & 370 & $\mathrm{U}$ & 370 & 370 & $\mathrm{U}$ & 370 & 370 & $\mathrm{U}$ & 370 & 370 & $\mathrm{U}$ & 370 \\
\hline \begin{tabular}{|l|} 
Diethylphthalate \\
\end{tabular} & 370 & $\mathrm{U}$ & 370 & 370 & $\mathrm{U}$ & 370 & 370 & $\mathrm{U}$ & 370 & 370 & $\mathrm{U}$ & 370 \\
\hline \begin{tabular}{|l|l|l|} 
Dimethylphthala \\
\end{tabular} & 370 & $U$ & 370 & 370 & $\mathrm{U}$ & 370 & 370 & $\mathrm{U}$ & 370 & 370 & $\mathrm{U}$ & 370 \\
\hline \begin{tabular}{|l} 
Di-n-butylphthalate \\
\end{tabular} & 370 & $\mathrm{U}$ & 370 & 370 & $\mathrm{U}$ & 370 & 370 & $\mathrm{U}$ & 370 & 370 & $\mathrm{U}$ & 370 \\
\hline \begin{tabular}{|l} 
Di-n-octylphthalate \\
\end{tabular} & 370 & $\mathrm{U}$ & 370 & 370 & $\mathrm{U}$ & 370 & 370 & $\mathrm{U}$ & 370 & 370 & $\mathrm{U}$ & 370 \\
\hline \begin{tabular}{|l} 
Fluoranthene \\
\end{tabular} & 370 & $\mathrm{U}$ & 370 & 47 & $\mathrm{~J}$ & 370 & 370 & $\mathrm{U}$ & 370 & 370 & $\mathrm{U}$ & 370 \\
\hline \begin{tabular}{|l} 
Fluorene \\
\end{tabular} & 370 & $\mathrm{U}$ & 370 & 370 & $U$ & 370 & 370 & $\mathrm{U}$ & 370 & 370 & $\mathrm{U}$ & 370 \\
\hline \begin{tabular}{|l|} 
Hexachlorobenzene \\
\end{tabular} & 370 & $\mathrm{U}$ & 370 & 370 & $\mathrm{U}$ & 370 & 370 & $\mathrm{U}$ & 370 & 370 & $\mathrm{U}$ & 370 \\
\hline \begin{tabular}{|l} 
Hexachlorobutadiene \\
\end{tabular} & 370 & $\mathrm{U}$ & 370 & 370 & $\mathrm{U}$ & 370 & 370 & $\mathrm{U}$ & 370 & 370 & $\mathrm{U}$ & 370 \\
\hline Hexachlorocyclopentadiene & 370 & $\mathrm{U}$ & 370 & 370 & $\mathrm{U}$ & 370 & 370 & $\mathrm{U}$ & 370 & 370 & $\mathrm{U}$ & 370 \\
\hline \begin{tabular}{|l} 
Hexachloroethane \\
\end{tabular} & 370 & $\mathrm{U}$ & 370 & 370 & $\mathrm{U}$ & 370 & 370 & $\mathrm{U}$ & 370 & 370 & $\mathrm{U}$ & 370 \\
\hline Indeno( $1,2,3$-cd)pyrene & 370 & $\mathrm{U}$ & 370 & 370 & $\mathrm{U}$ & 370 & 370 & $\mathrm{U}$ & 370 & 370 & $\mathrm{U}$ & 370 \\
\hline \begin{tabular}{|l} 
Isophorone \\
\end{tabular} & 370 & $\mathrm{U}$ & 370 & 370 & $\mathrm{U}$ & 370 & 370 & $\mathrm{U}$ & 370 & 370 & $\mathrm{U}$ & 370 \\
\hline \begin{tabular}{|l} 
Naphthalene \\
\end{tabular} & 370 & $\mathrm{U}$ & 370 & 370 & $\mathrm{U}$ & 370 & 370 & $\mathrm{U}$ & 370 & 370 & $\mathrm{U}$ & 370 \\
\hline \begin{tabular}{|l} 
Nitrobenzene \\
\end{tabular} & 370 & $\mathrm{U}$ & 370 & 370 & $\mathrm{U}$ & 370 & 370 & $\mathrm{U}$ & 370 & 370 & $\mathrm{U}$ & 370 \\
\hline \begin{tabular}{|l} 
N-Nitroso-di-n-dipropylamine \\
\end{tabular} & 370 & $\mathrm{U}$ & 370 & 370 & $\mathrm{U}$ & 370 & 370 & $\mathrm{U}$ & 370 & 370 & $\mathrm{U}$ & 370 \\
\hline N-Nitrosodiphenylamine & 370 & $\mathrm{U}$ & 370 & 370 & $\mathrm{U}$ & 370 & 370 & $U$ & 370 & 370 & $\mathrm{U}$ & 370 \\
\hline \begin{tabular}{|l} 
Pentachlorophenol \\
\end{tabular} & 930 & $\mathrm{U}$ & 930 & 930 & $\mathrm{U}$ & 930 & 920 & $\mathrm{U}$ & 920 & 920 & $\mathrm{U}$ & 920 \\
\hline \begin{tabular}{|l} 
Phenanthrene \\
\end{tabular} & 370 & $\mathrm{U}$ & 370 & 370 & $\mathrm{U}$ & 370 & 370 & $\mathrm{U}$ & 370 & 370 & $\mathrm{U}$ & 370 \\
\hline \begin{tabular}{|l} 
Phenol \\
\end{tabular} & 370 & $\mathrm{U}$ & 370 & 370 & $\mathrm{U}$ & 370 & 370 & $\mathrm{U}$ & 370 & 370 & $\mathrm{U}$ & 370 \\
\hline \begin{tabular}{|l|l} 
Pyrene \\
\end{tabular} & 370 & $\mathrm{U}$ & 370 & 370 & $\mathrm{U}$ & 370 & 370 & $\mathrm{U}$ & 370 & 370 & $\mathrm{U}$ & 370 \\
\hline
\end{tabular}


Table A-2. 120-F-1 Northwest Excavation Sampling Results from Initial Verification Samples. (8 Pages)

\begin{tabular}{|c|c|c|c|c|c|c|c|c|c|c|}
\hline \multirow[t]{2}{*}{ Constituent } & \multicolumn{3}{|c|}{$\begin{array}{c}\text { J16351 } \\
\text { Sample Location NW-8 } \\
\text { Sample Date 12/3/08 }\end{array}$} & \multicolumn{3}{|c|}{$\begin{array}{c}\text { J16352 } \\
\text { Sample Location NW-9 } \\
\text { Sample Date 12/3/08 }\end{array}$} & \multicolumn{3}{|c|}{$\begin{array}{c}\text { J16353 } \\
\text { Sample Location NW- } \\
10 \\
\text { Sample Date 12/3/08 }\end{array}$} & \\
\hline & $\mu \mathrm{g} / \mathrm{kg}$ & Q & PQL & $\mu \mathrm{g} / \mathrm{kg}$ & $Q$ & PQL & $\mu \mathrm{g} / \mathrm{kg}$ & $Q$ & PQL & \\
\hline \multicolumn{11}{|c|}{ Polychlorinated Biphenyls } \\
\hline Aroclor-1016 & 7000 & $\mathrm{U}$ & 7000 & 7600 & $\mathrm{U}$ & 7600 & 15 & $\mathrm{U}$ & 15 & \\
\hline Aroclor-1221 & 7000 & $\mathrm{U}$ & 7000 & 7600 & $\mathrm{U}$ & 7600 & 15 & $\mathrm{U}$ & 15 & \\
\hline Aroclor -1232 & 7000 & $\mathrm{U}$ & 7000 & 7600 & $\mathrm{U}$ & 7600 & 15 & $\mathrm{U}$ & 15 & \\
\hline Aroclor-1242 & 7000 & $\mathrm{U}$ & 7000 & 7600 & $\mathrm{U}$ & 7600 & 15 & $\mathrm{U}$ & 15 & \\
\hline Aroclor- 1248 & 7000 & $\mathrm{U}$ & 7000 & 7600 & $\mathrm{U}$ & 7600 & 15 & $\mathrm{U}$ & 15 & \\
\hline Aroclor- 1254 & 7000 & $\mathrm{U}$ & 7000 & 7600 & $\mathrm{U}$ & 7600 & 15 & $\mathrm{U}$ & 15 & \\
\hline Aroclor -1260 & 7000 & U & 7000 & 7600 & $\mathrm{U}$ & 7600 & 15 & $\mathrm{U}$ & 15 & \\
\hline \multicolumn{11}{|c|}{ Pesticides } \\
\hline Aldrin & 70 & U] & 70 & 9.5 & $\mathrm{U}$ & 9.5 & 1.5 & UD & 1.5 & \\
\hline Alpha-BHC & 70 & $\mathrm{U}$ & 70 & 9.5 & $\mathrm{U}$ & 9.5 & 1.5 & UD & 1.5 & \\
\hline Alpha-Chlordane & 12000 & $D$ & 70 & 1100 & $D$ & 9.5 & 43 & $D$ & 1.5 & \\
\hline Beta-BHC & 70 & $\mathrm{U}$ & 70 & 9.5 & $\mathrm{U}$ & 9.5 & 1.5 & UD & 1.5 & \\
\hline Delta-BHC & 70 & U & 70 & 9.5 & $\mathrm{U}$ & 9.5 & 1.5 & UD & 1.5 & \\
\hline Dichlorodiphenyldichloroethane & 530 & $\mathrm{x}$ & 70 & 420 & $\mathrm{X}$ & 9.5 & 1.5 & UD & 1.5 & \\
\hline Dichlorodiphenyldichloroethylene & 70 & $\mathrm{U}$ & 70 & 9.5 & $\mathrm{U}$ & 9.5 & 1.5 & UD & 1.5 & \\
\hline Dichlorodiphenyltrichloroethane & 70 & $\mathrm{U}$ & 70 & 9.5 & $\mathrm{U}$ & 9.5 & 1.5 & UD & 1.5 & \\
\hline Dieldrin & 440 & $\mathrm{X}$ & 70 & 34 & $\mathrm{JX}$ & 9.5 & 1.5 & UD & 1.5 & \\
\hline Endosulfan I & 88 & $\mathrm{JD}$ & 70 & 9.5 & $\mathrm{U}$ & 9.5 & 1.5 & UD & 1.5 & \\
\hline Endosulfan II & 70 & $\mathrm{U}$ & 70 & 9.5 & $\mathrm{U}$ & 9.5 & 1.5 & UD & 1.5 & \\
\hline Endosulfan sulfate & 70 & $\mathrm{U}$ & 70 & 9.5 & $\mathrm{U}$ & 9.5 & 1.5 & UD & 1.5 & \\
\hline Endrin & 70 & $\mathrm{U}$ & 70 & 47 & $\mathrm{D}$ & 9.5 & 2.5 & $\mathrm{JD}$ & 1.5 & \\
\hline Endrin aldehyde & 70 & U & 70 & 9.5 & $U$ & 9.5 & 1.5 & UD & 1.5 & \\
\hline Endrin ketone & 70 & $\mathrm{U}$ & 70 & 9.5 & $\mathrm{U}$ & 9.5 & 1.5 & UD & 1.5 & \\
\hline Gamma-BHC (Lindane) & 70 & $\mathrm{U}$ & 70 & 9.5 & $\mathrm{U}$ & 9.5 & 1.5 & UD & 1.5 & \\
\hline gamma-Chlordane & 12000 & $\mathrm{D}$ & 70 & 880 & $\mathrm{D}$ & 9.5 & 40 & $\mathrm{D}$ & 1.5 & \\
\hline Heptachlor & 5700 & D & 70 & 370 & $\mathrm{D}$ & 9.5 & 20 & $\bar{D}$ & 1.5 & \\
\hline Heptachlor epoxide & 70 & $\mathrm{U}$ & 70 & 9.5 & $\mathrm{U}$ & 9.5 & 1.5 & UD & 1.5 & \\
\hline Methoxychlor & 70 & $\mathrm{U}$ & 70 & 9.5 & $\mathrm{U}$ & 9.5 & 1.5 & UD & 1.5 & \\
\hline Toxaphene & 700 & $\mathrm{U}$ & 700 & 95 & $\mathrm{U}$ & 95 & 15 & UD & 15 & \\
\hline \multicolumn{11}{|c|}{ Semivolatile Organic Analytes } \\
\hline 1,2,4-Trichlorobenzene & 350 & $\mathrm{U}$ & 350 & 380 & $\mathrm{U}$ & 380 & 370 & $\mathrm{U}$ & 370 & \\
\hline 1,2-Dichlorobenzene & 350 & $\mathrm{U}$ & 350 & 380 & $\mathrm{U}$ & 380 & 370 & $\mathrm{U}$ & 370 & \\
\hline 1,3-Dichlorobenzene & 350 & U & 350 & 380 & $\mathrm{U}$ & 380 & 370 & $\mathrm{U}$ & 370 & \\
\hline 1,4-Dichlorobenzene & 350 & $\mathrm{U}$ & 350 & 380 & $\mathrm{U}$ & 380 & 370 & $\mathrm{U}$ & 370 & \\
\hline 2,4,5-Trichlorophenol & 880 & $\mathrm{U}$ & 880 & 950 & $\mathrm{U}$ & 950 & 930 & $\bar{U}$ & 930 & \\
\hline 2,4,6-Trichlorophenol & 350 & $\mathrm{U}$ & 350 & 380 & $\mathrm{U}$ & 380 & 370 & $\mathrm{U}$ & 370 & \\
\hline 2,4-Dichlorophenol & 350 & $\mathrm{U}$ & 350 & 380 & $\mathrm{U}$ & 380 & 370 & $\mathrm{U}$ & 370 & \\
\hline 2,4-Dimethylphenol & 350 & $\mathrm{U}$ & 350 & 380 & $U$ & 380 & 370 & $\bar{U}$ & 370 & \\
\hline 2,4-Dinitrophenol & 880 & $\mathrm{U}$ & 880 & 950 & $\mathrm{U}$ & 950 & 930 & $\mathrm{U}$ & 930 & \\
\hline 2,4-Dinitrotoluene & 350 & $\mathrm{U}$ & 350 & 380 & $\mathrm{U}$ & 380 & 370 & $\mathrm{U}$ & 370 & \\
\hline 2,6-Dinitrotoluene & 350 & U & 350 & 380 & $\mathrm{U}$ & 380 & 370 & $\mathrm{U}$ & 370 & \\
\hline 2-Chloronaphthalene & 350 & $\mathrm{U}$ & 350 & 380 & $\mathrm{U}$ & 380 & 370 & $\bar{U}$ & 370 & \\
\hline 2-Chlorophenol & 350 & $\mathrm{U}$ & 350 & 380 & $\mathrm{U}$ & 380 & 370 & $\mathrm{U}$ & 370 & \\
\hline 2-Methylnaphthalene & 350 & $\mathrm{U}$ & 350 & 380 & $\mathrm{U}$ & 380 & 370 & $\mathrm{U}$ & 370 & \\
\hline 2-Methylphenol (cresol, o-) & 350 & $\mathrm{U}$ & 350 & 380 & $\mathrm{U}$ & 380 & 370 & $\mathrm{U}$ & 370 & \\
\hline 2-Nitroaniline & 880 & $\mathrm{U}$ & 880 & 950 & $\mathrm{U}$ & 950 & 930 & $\mathrm{U}$ & 930 & \\
\hline 2-Nitrophenol & 350 & $\mathrm{U}$ & 350 & 380 & $\mathrm{U}$ & 380 & 370 & $\mathrm{U}$ & 370 & \\
\hline
\end{tabular}


Table A-2. 120-F-1 Northwest Excavation Sampling Results from Initial Verification Samples. (8 Pages)

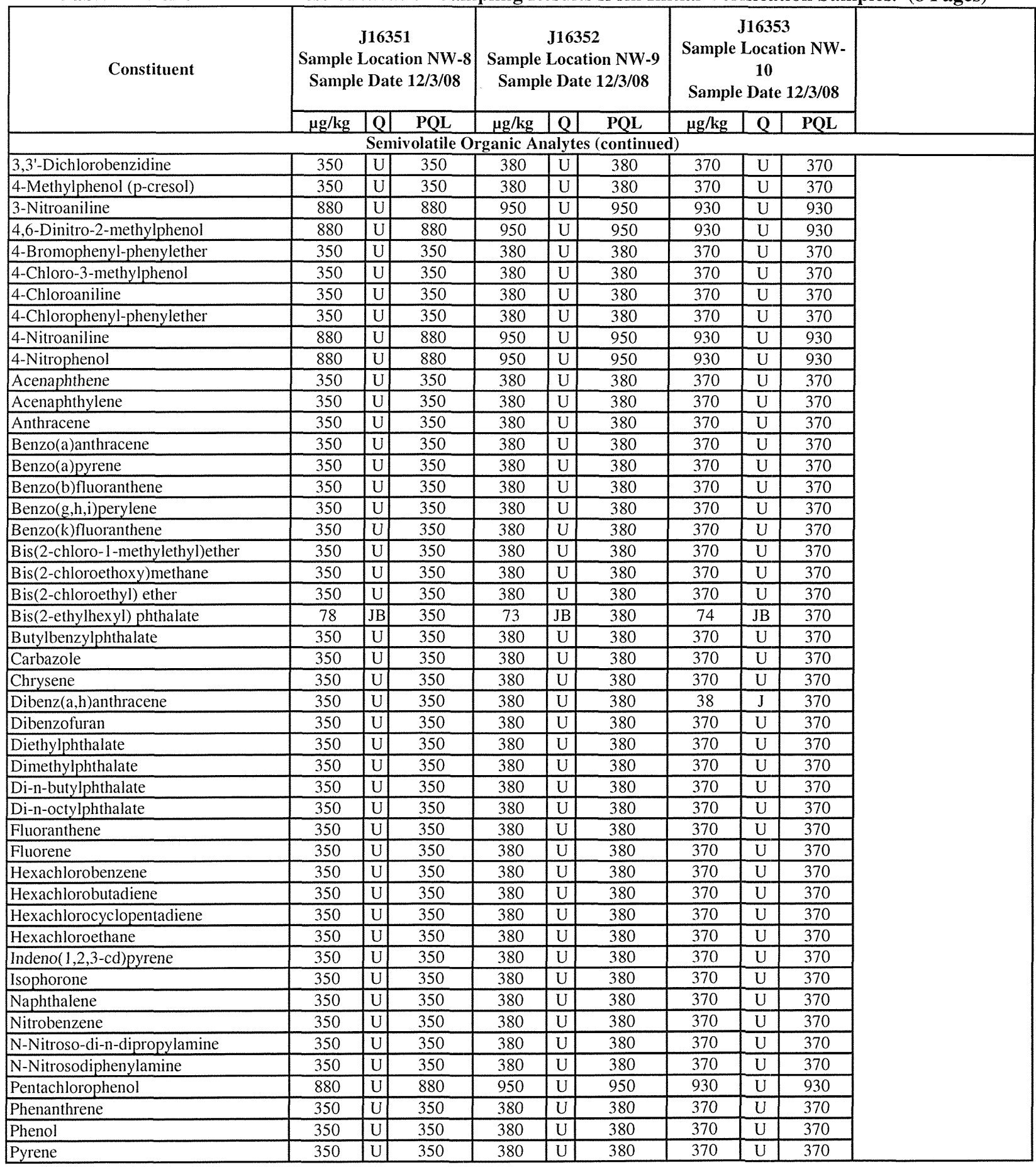


Table A-3. 120-F-1 Northwest Excavtion Pre-Verification Sampling Results. (2 Pages)

\begin{tabular}{|c|c|c|c|c|c|c|c|c|c|c|c|c|c|c|c|}
\hline \multirow[t]{2}{*}{ Constituent } & \multicolumn{3}{|c|}{$\begin{array}{c}\text { J169K6 } \\
\text { Sample Location NW-1 } \\
\text { Sample Date } 2 / 4 / 08\end{array}$} & \multicolumn{3}{|c|}{$\begin{array}{c}\text { J169K7 } \\
\text { Sample Location NW-2 } \\
\text { Sample Date 2/4/08 }\end{array}$} & \multicolumn{3}{|c|}{$\begin{array}{c}\text { J169K8 } \\
\text { Sample Location NW-3 } \\
\text { Sample Date 2/4/08 }\end{array}$} & \multicolumn{3}{|c|}{$\begin{array}{c}\text { J169K9 } \\
\text { Sample Location NW-4 } \\
\text { Sample Date } 2 / 4 / 08\end{array}$} & \multicolumn{3}{|c|}{$\begin{array}{c}\text { J169L0 } \\
\text { Sample Location NW-5 } \\
\text { Sample Date 2/4/08 }\end{array}$} \\
\hline & $\mu \mathrm{g} / \mathrm{kg}$ & $Q$ & PQL & $\mu \mathrm{g} / \mathrm{kg}$ & Q & PQL & $\mu \mathrm{g} / \mathrm{kg}$ & $\mathbf{Q}$ & PQL & $\mu \mathrm{g} / \mathrm{kg}$ & $\mathbf{Q}$ & $\mathrm{PQL}$ & $\mu \mathrm{g} / \mathrm{kg}$ & Q & PQL \\
\hline \multicolumn{16}{|c|}{ Polychlorinated Biphenyls } \\
\hline Aroclor-1016 & 14 & $\mathrm{U}$ & 14 & 14 & $\mathrm{U}$ & 14 & 14 & $\mathrm{U}$ & 14 & 14 & $\mathrm{U}$ & 14 & 14 & $\mathrm{U}$ & 14 \\
\hline Aroclor -1221 & 14 & $\mathrm{U}$ & 14 & 14 & $\mathrm{U}$ & 14 & 14 & $\mathrm{U}$ & 14 & 14 & $\mathrm{U}$ & 14 & 14 & $\mathrm{U}$ & 14 \\
\hline Aroclor-1232 & 14 & $\mathrm{U}$ & 14 & 14 & $\mathrm{U}$ & 14 & 14 & $\mathrm{U}$ & 14 & 14 & $\mathrm{U}$ & 14 & 14 & $\mathrm{U}$ & 14 \\
\hline Aroclor-1242 & 14 & $\mathrm{U}$ & 14 & 14 & $\mathrm{U}$ & 14 & 14 & $\mathrm{U}$ & 14 & 14 & $\mathrm{U}$ & 14 & 14 & $\mathrm{U}$ & 14 \\
\hline Aroclor-1248 & 14 & $\mathrm{U}$ & 14 & 14 & $\mathrm{U}$ & 14 & 14 & $\mathrm{U}$ & 14 & 14 & $\mathrm{U}$ & 14 & 14 & $\mathrm{U}$ & 14 \\
\hline Aroclor-1254 & 14 & $\mathrm{U}$ & 14 & 14 & U & 14 & 14 & $\mathrm{U}$ & 14 & 14 & $\mathrm{U}$ & 14 & 14 & 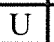 & 14 \\
\hline Aroclor-1260 & 14 & $\mathrm{U}$ & 14 & 14 & $\mathrm{U}$ & 14 & 14 & $\mathrm{U}$ & 14 & 14 & $\mathrm{U}$ & 14 & 14 & $\mathrm{U}$ & 14 \\
\hline \multicolumn{16}{|c|}{ Pesticides } \\
\hline Aldrin & 1.5 & $\mathrm{U}$ & 1.5 & 2.3 & $\mathrm{~J}$ & 1.6 & 1.4 & $\mathrm{U}$ & 1.4 & 1.6 & $\mathrm{U}$ & 1.6 & 1.4 & $\mathrm{U}$ & 1.4 \\
\hline Alpha-BHC & 1.5 & $\mathrm{U}$ & 1.5 & 1.6 & $\mathrm{U}$ & 1.6 & 1.4 & $\mathrm{U}$ & 1.4 & 1.6 & $\mathrm{U}$ & 1.6 & 1.4 & $\mathrm{U}$ & 1.4 \\
\hline Alpha-Chlordane & 12 & & 1.5 & 260 & & 1.6 & 6.1 & $\mathrm{~J}$ & 1.4 & 190 & & 1.6 & 50 & & 1.4 \\
\hline Beta-BHC & 1.5 & $\mathrm{U}$ & 1.5 & 1.8 & $\mathrm{~J}, \mathrm{I}$ & 1.6 & 1.4 & $\mathrm{U}$ & 1.4 & 1.6 & $\mathrm{U}$ & 1.6 & 1.4 & $\mathrm{U}$ & 1.4 \\
\hline Delta-BHC & 1.5 & $\mathrm{U}$ & 1.5 & 1.4 & $\mathrm{U}$ & 1.6 & 1.4 & $\mathrm{U}$ & 1.4 & 1.6 & $\mathrm{U}$ & 1.6 & 1.4 & $\mathrm{U}$ & 1.4 \\
\hline Dichlorodiphenyldichloroethane & 1.5 & $\mathrm{U}$ & 1.5 & 16 & $\mathrm{I}$ & 1.6 & 1.4 & $\mathrm{U}$ & 1.4 & 9.3 & $\mathrm{I}$ & 1.6 & 1.4 & $\mathrm{U}$ & 1.4 \\
\hline Dichlorodiphenyldichloroethylene & 1.5 & $\mathrm{U}$ & 1.5 & 1.6 & $\mathrm{U}$ & 1.6 & 1.4 & $\mathrm{U}$ & 1.4 & 1.6 & $\mathrm{U}$ & 1.6 & 1.4 & $\mathrm{U}$ & 1.4 \\
\hline Dichlorodiphenyltrichloroethane & 1.5 & $\mathrm{U}$ & 1.5 & 20 & & 1.6 & 1.4 & $\mathrm{U}$ & 1.4 & 2.6 & $\mathrm{~J}$ & 1.6 & 1.4 & $\mathrm{U}$ & 1.4 \\
\hline Dieldrin & 1.4 & $\mathrm{U}$ & 1.5 & 13 & & 1.6 & 1.4 & $\mathrm{U}$ & 1.4 & 7.8 & & 1.6 & 2.2 & $\mathrm{~J}$ & 1.4 \\
\hline Endosulfan I & 1.5 & $\mathrm{U}$ & 1.5 & 1.6 & $\mathrm{U}$ & 1.6 & 1.4 & $\mathrm{U}$ & 1.4 & 1.6 & $\mathrm{U}$ & 1.6 & 1.4 & $\mathrm{U}$ & 1.4 \\
\hline Endosulfan II & 1.4 & $\mathrm{U}$ & 1.5 & 1.6 & $\mathrm{U}$ & 1.6 & 1.4 & $\mathrm{U}$ & 1.4 & 1.6 & $\mathrm{U}$ & 1.6 & 1.4 & $\mathrm{U}$ & 1.4 \\
\hline Endosulfan sulfate & 1.4 & $\mathrm{U}$ & 1.5 & 1.6 & $\mathrm{U}$ & 1.6 & 1.4 & $\mathrm{U}$ & 1.4 & 1.6 & $\mathrm{U}$ & 1.6 & 1.4 & $\mathrm{U}$ & 1.4 \\
\hline Endrin & 1.5 & $\mathrm{U}$ & 1.5 & 20 & & 1.6 & 1.4 & $\mathrm{U}$ & 1.4 & 12 & & 1.6 & 3.6 & $\mathrm{~J}$ & 1.4 \\
\hline Endrin aldehyde & 1.5 & $\mathrm{U}$ & 1.5 & 3.0 & $\mathrm{~J}, \mathrm{I}$ & 1.6 & 1.4 & $\mathrm{U}$ & 1.4 & 1.6 & $\mathrm{U}$ & 1.6 & 1.4 & $\mathrm{U}$ & 1.4 \\
\hline Endrin ketone & 1.5 & $\mathrm{U}$ & 1.5 & 1.6 & $\mathrm{U}$ & 1.6 & 1.4 & $\mathrm{U}$ & 1.4 & 1.6 & $\mathrm{U}$ & 1.6 & 1.4 & $\mathrm{U}$ & 1.4 \\
\hline Gamma-BHC (Lindane) & 1.5 & $\mathrm{U}$ & 1.5 & 1.6 & $\mathrm{U}$ & 1.6 & 1.4 & $\mathrm{U}$ & 1.4 & 1.6 & $\mathrm{U}$ & 1.6 & 1.4 & $\mathrm{U}$ & 1.4 \\
\hline gamma-Chlordane & 13 & & 1.5 & 280 & & 1.6 & 5.4 & $\mathrm{~J}$ & 1.4 & 190 & & 1.6 & 42 & & 1.4 \\
\hline Heptachlor & 1.6 & $\mathrm{~J}$ & 1.5 & 110 & & 1.6 & 1.4 & $\mathrm{U}$ & 1.4 & 87 & & 1.6 & 11 & & 1.4 \\
\hline Heptachlor epoxide & 1.5 & $\mathrm{U}$ & 1.5 & 1.6 & $\mathrm{U}$ & 1.6 & 1.4 & $\mathrm{U}$ & 1.4 & 1.6 & $\mathrm{U}$ & 1.6 & 2.0 & $\mathrm{~J}, \mathrm{I}$ & 1.4 \\
\hline \begin{tabular}{|l|} 
Methoxychlor \\
\end{tabular} & 1.5 & $\mathrm{U}$ & 1.5 & 1.6 & $\mathrm{U}$ & 1.6 & 1.4 & $\mathrm{U}$ & 1.4 & 1.6 & $\mathrm{U}$ & 1.6 & 1.4 & $\mathrm{U}$ & 1.4 \\
\hline Toxaphene & 15 & $\mathrm{U}$ & 15 & 16 & $\mathrm{U}$ & 16 & 14 & $\mathrm{U}$ & 14 & 16 & $\mathrm{U}$ & 16 & 14 & $\mathrm{U}$ & 14 \\
\hline
\end{tabular}


Table A-3. 120-F-1 Northwest Excavtion Pre-Verification Sampling Results. (2 Pages)

\begin{tabular}{|c|c|c|c|c|c|c|c|c|c|c|c|c|c|c|c|}
\hline \multirow[t]{2}{*}{ Constituent } & \multicolumn{3}{|c|}{$\begin{array}{c}\text { J169L1 } \\
\text { Sample Location NW-6 } \\
\text { Sample Date 2/4/08 }\end{array}$} & \multicolumn{3}{|c|}{$\begin{array}{c}\text { J169L2 } \\
\text { Sample Location NW-7 } \\
\text { Sample Date 2/4/08 }\end{array}$} & \multicolumn{3}{|c|}{\begin{tabular}{|c|} 
J169L3 \\
Sample Location NW-8 \\
Sample Date $2 / 4 / 08$
\end{tabular}} & \multicolumn{3}{|c|}{$\begin{array}{c}\text { J169L4 } \\
\text { Sample Location NW-9 } \\
\text { Sample Date 2/4/08 }\end{array}$} & \multicolumn{3}{|c|}{$\begin{array}{c}\text { J169L5 } \\
\text { Sample Location NW- } \\
10 \\
\text { Sample Date 2/4/08 }\end{array}$} \\
\hline & $\mu \mathrm{g} / \mathrm{kg}$ & $Q$ & PQL & $\mu \mathrm{g} / \mathrm{kg}$ & $Q$ & PQL & $\mu \mathrm{g} / \mathrm{kg}$ & $Q$ & PQL & $\mu \mathrm{g} / \mathrm{kg}$ & Q & PQL & $\mu \mathrm{g} / \mathrm{kg}$ & Q & PQL \\
\hline \multicolumn{16}{|c|}{ Polychlorinated Biphenyls } \\
\hline Aroclor-1016 & 14 & $\mathrm{U}$ & 14 & 14 & $\mathrm{U}$ & 14 & 14 & $\mathrm{U}$ & 14 & 14 & $\mathrm{U}$ & 14 & 15 & $\mathrm{U}$ & 15 \\
\hline Aroclor-1221 & 14 & $\mathrm{U}$ & 14 & 14 & $\mathrm{U}$ & 14 & 14 & $\mathrm{U}$ & 14 & 14 & $\mathrm{U}$ & 14 & 15 & $\mathrm{U}$ & 15 \\
\hline Aroclor-1232 & 14 & $\mathrm{U}$ & 14 & 14 & $\mathrm{U}$ & 14 & 14 & $\mathrm{U}$ & 14 & 14 & $\mathrm{U}$ & 14 & 15 & $\mathrm{U}$ & 15 \\
\hline Aroclor-1242 & 14 & $\mathrm{U}$ & 14 & 14 & $\mathrm{U}$ & 14 & 14 & $\mathrm{U}$ & 14 & 14 & $\mathrm{U}$ & 14 & 15 & U & 15 \\
\hline Aroclor-1248 & 14 & $\mathrm{U}$ & 14 & 14 & $\mathrm{U}$ & 14 & 14 & $\mathrm{U}$ & 14 & 14 & $\mathrm{U}$ & 14 & 15 & $\mathrm{U}$ & 15 \\
\hline Aroclor-1254 & 14 & $\mathrm{U}$ & 14 & 14 & $\mathrm{U}$ & 14 & 14 & $\mathrm{U}$ & 14 & 14 & $\mathrm{U}$ & 14 & 23 & & 15 \\
\hline Aroclor -1260 & 14 & $\mathrm{U}$ & 14 & 14 & $\mathrm{U}$ & 14 & 14 & $\mathrm{U}$ & 14 & 14 & $\mathrm{U}$ & 14 & 9.8 & $\mathrm{~J}$ & 15 \\
\hline \multicolumn{16}{|c|}{ Pesticides } \\
\hline Aldrin & 1.4 & $\mathrm{U}$ & 1.4 & 1.4 & $\mathrm{U}$ & 1.4 & 1.4 & $\mathrm{U}$ & 1.4 & 1.4 & $\mathrm{U}$ & 1.4 & 1.4 & $\mathrm{U}$ & 1.5 \\
\hline Alpha-BHC & 1.4 & $\mathrm{U}$ & 1.4 & 1.4 & $\mathrm{U}$ & 1.4 & 1.4 & $\mathrm{U}$ & 1.4 & 1.4 & $\mathrm{U}$ & 1.4 & 1.4 & $\mathrm{U}$ & 1.5 \\
\hline Alpha-Chlordane & 2.7 & $\mathrm{~J}$ & 1.4 & 1.4 & $\mathrm{U}$ & 1.4 & 4.7 & $\mathrm{~J}$ & 1.4 & 1.4 & $\mathrm{U}$ & 1.4 & 1.4 & $\mathrm{U}$ & 1.5 \\
\hline Beta-BHC & 1.4 & $\mathrm{U}$ & 1.4 & 1.4 & $\mathrm{U}$ & 1.4 & 1.4 & $\mathrm{U}$ & 1.4 & 1.4 & $\mathrm{U}$ & 1.4 & 1.4 & $\mathrm{U}$ & 1.5 \\
\hline Delta-BHC & 1.4 & $\mathrm{U}$ & 1.4 & 1.4 & $\mathrm{U}$ & 1.4 & 1.4 & $\mathrm{U}$ & 1.4 & 1.4 & $\mathrm{U}$ & 1.4 & 1.4 & $\mathrm{U}$ & 1.5 \\
\hline Dichlorodiphenyldichloroethane & 1.4 & $\mathrm{U}$ & 1.4 & 1.4 & $\mathrm{U}$ & 1.4 & 1.4 & $\mathrm{U}$ & 1.4 & 1.4 & $\mathrm{U}$ & 1.4 & 1.4 & $\mathrm{U}$ & 1.5 \\
\hline Dichlorodiphenyldichloroethylene & 1.4 & $\mathrm{U}$ & 1.4 & 1.4 & $\mathrm{U}$ & 1.4 & 1.4 & $\mathrm{U}$ & 1.4 & 1.4 & $\mathrm{U}$ & 1.4 & 1.4 & $\mathrm{U}$ & 1.5 \\
\hline Dichlorodiphenyltrichloroethane & 1.4 & $\mathrm{U}$ & 1.4 & 1.4 & $\mathrm{U}$ & 1.4 & 1.4 & $\mathrm{U}$ & 1.4 & 1.4 & $\mathrm{U}$ & 1.4 & 1.4 & $\mathrm{U}$ & 1.5 \\
\hline Dieldrin & 1.4 & $\mathrm{U}$ & 1.4 & 1.4 & $\mathrm{U}$ & 1.4 & 1.4 & $\mathrm{U}$ & 1.4 & 1.4 & $\mathrm{U}$ & 1.4 & 1.4 & $\mathrm{U}$ & 1.5 \\
\hline Endosulfan I & 1.4 & $\mathrm{U}$ & 1.4 & 1.4 & $\mathrm{U}$ & 1.4 & 1.4 & $\mathrm{U}$ & 1.4 & 1.4 & $\mathrm{U}$ & 1.4 & 1.4 & $\mathrm{U}$ & 1.5 \\
\hline Endosulfan II & 1.4 & $\mathrm{U}$ & 1.4 & 1.4 & $\mathrm{U}$ & 1.4 & 1.4 & $\mathrm{U}$ & 1.4 & 1.4 & $\mathrm{U}$ & 1.4 & 1.4 & $\mathrm{U}$ & 1.5 \\
\hline Endosulfan sulfate & 1.4 & $\mathrm{U}$ & 1.4 & 1.4 & $\mathrm{U}$ & 1.4 & 1.4 & $\mathrm{U}$ & 1.4 & 1.4 & $\mathrm{U}$ & 1.4 & 1.4 & $\mathrm{U}$ & 1.5 \\
\hline Endrin & 1.4 & $\mathrm{U}$ & 1.4 & 1.4 & $\mathrm{U}$ & 1.4 & 1.4 & $\mathrm{U}$ & 1.4 & 1.4 & $\mathrm{U}$ & 1.4 & 1.4 & $\mathrm{U}$ & 1.5 \\
\hline Endrin aldehyde & 1.4 & $\mathrm{U}$ & 1.4 & 1.4 & $\mathrm{U}$ & 1.4 & 1.4 & $\mathrm{U}$ & 1.4 & 1.4 & $\mathrm{U}$ & 1.4 & 1.4 & $\mathrm{U}$ & 1.5 \\
\hline Endrin ketone & 1.4 & $\mathrm{U}$ & 1.4 & 1.4 & $\mathrm{U}$ & 1.4 & 1.4 & $\mathrm{U}$ & 1.4 & 1.4 & $\mathrm{U}$ & 1.4 & 1.4 & $\mathrm{U}$ & 1.5 \\
\hline Gamma-BHC (Lindane) & 1.4 & $\mathrm{U}$ & 1.4 & 1.4 & $\mathrm{U}$ & 1.4 & 1.4 & $\mathrm{U}$ & 1.4 & 1.4 & $\mathrm{U}$ & 1.4 & 1.4 & $\mathrm{U}$ & 1.5 \\
\hline gamma-Chlordane & 2.6 & $\mathrm{~J}$ & 1.4 & 1.4 & $\mathrm{U}$ & 1.4 & 4.9 & $\mathrm{~J}$ & 1.4 & 1.4 & $\mathrm{U}$ & 1.4 & 1.4 & $\mathrm{U}$ & 1.5 \\
\hline Heptachlor & 1.4 & $\mathrm{U}$ & 1.4 & 1.4 & $\mathrm{U}$ & 1.4 & 2.6 & $\mathrm{~J}$ & 1.4 & 1.4 & $\mathrm{U}$ & 1.4 & 1.4 & $\mathrm{U}$ & 1.5 \\
\hline Heptachlor epoxide & 1.4 & $\mathrm{U}$ & 1.4 & 1.4 & $\mathrm{U}$ & 1.4 & 1.4 & $\mathrm{U}$ & 1.4 & 1.4 & $\mathrm{U}$ & 1.4 & 1.4 & $\mathrm{U}$ & 1.5 \\
\hline Methoxychlor & 1.4 & $\mathrm{U}$ & 1.4 & 1.4 & $\mathrm{U}$ & 1.4 & 1.4 & $\mathrm{U}$ & 1.4 & 1.4 & $\mathrm{U}$ & 1.4 & 1.4 & $\mathrm{U}$ & 1.5 \\
\hline Toxaphene & 14 & $\mathrm{U}$ & 14 & 14 & $\mathrm{U}$ & 14 & 14 & $\mathrm{U}$ & 14 & 14 & $\mathrm{U}$ & 14 & 14 & $\mathrm{U}$ & 15 \\
\hline
\end{tabular}


Table A-4. 120-F-1 Northwest Excavation Pre-Verification Sampling Results. (2 Pages)

\begin{tabular}{|c|c|c|c|c|c|c|c|c|c|c|c|c|c|c|c|}
\hline \multirow[t]{2}{*}{ Constituent } & \multicolumn{3}{|c|}{$\begin{array}{c}\text { J16B47 } \\
\text { Sample Location NW-1 } \\
\text { Sample Date 2/19/08 }\end{array}$} & \multicolumn{3}{|c|}{$\begin{array}{c}\text { J16B48 } \\
\text { Sample Location NW-2 } \\
\text { Sample Date } 2 / 19 / 08\end{array}$} & \multicolumn{3}{|c|}{$\begin{array}{c}\text { J16B49 } \\
\text { Sample Location NW-3 } \\
\text { Sample Date 2/19/08 }\end{array}$} & \multicolumn{3}{|c|}{$\begin{array}{c}\text { J16B50 } \\
\text { Sample Location NW-4 } \\
\text { Sample Date } 2 / 19 / 08\end{array}$} & \multicolumn{3}{|c|}{$\begin{array}{c}\text { J16B51 } \\
\text { Sample Location NW-5 } \\
\text { Sample Date } 2 / 19 / 08\end{array}$} \\
\hline & $\mu \mathrm{g} / \mathrm{kg}$ & $Q$ & PQL & $\mu \mathrm{g} / \mathrm{kg}$ & $Q$ & $\mathrm{PQL}$ & $\mu \mathrm{g} / \mathrm{kg}$ & $Q$ & PQL & $\mu \mathrm{g} / \mathrm{kg}$ & $Q$ & $\mathrm{PQL}$ & $\mu \mathrm{g} / \mathrm{kg}$ & $\mathbf{Q}$ & PQL \\
\hline \multicolumn{16}{|c|}{ Pesticides } \\
\hline Aldrin & 2.0 & $\mathrm{U}$ & 2.0 & 2.1 & $\mathrm{U}$ & 2.1 & 2.1 & $\mathrm{U}$ & 2.1 & 2.1 & $\mathrm{U}$ & 2.1 & 2.0 & $\mathrm{U}$ & 2.0 \\
\hline Alpha-BHC & 2.0 & $\mathrm{U}$ & 2.0 & 2.1 & $\mathrm{U}$ & 2.1 & 2.1 & $\mathrm{U}$ & 2.1 & 2.1 & $\mathrm{U}$ & 2.1 & 2.0 & $\mathrm{U}$ & 2.0 \\
\hline Alpha-Chlordane & 2.0 & $\mathrm{U}$ & 2.0 & 2.1 & $\mathrm{U}$ & 2.1 & 2.1 & $\mathrm{U}$ & 2.1 & 81 & & 2.1 & 2.0 & $\mathrm{U}$ & 2.0 \\
\hline Beta-BHC & 2.0 & $\mathrm{U}$ & 2.0 & 2.1 & $\mathrm{U}$ & 2.1 & 2.1 & $\mathrm{U}$ & 2.1 & 2.1 & $\mathrm{U}$ & 2.1 & 2.0 & $\mathrm{U}$ & 2.0 \\
\hline Delta-BHC & 2.0 & $\mathrm{U}$ & 2.0 & 2.1 & $\mathrm{U}$ & 2.1 & 2.1 & $\mathrm{U}$ & 2.1 & 2.1 & $\mathrm{U}$ & 2.1 & 2.0 & $\mathrm{U}$ & 2.0 \\
\hline Dichlorodiphenyldichloroethane & 2.0 & $\mathrm{U}$ & 2.0 & 2.1 & $\mathrm{U}$ & 2.1 & 2.1 & $\mathrm{U}$ & 2.1 & 2.1 & $\mathrm{U}$ & 2.1 & 2.0 & $\mathrm{U}$ & 2.0 \\
\hline Dichlorodiphenyldichloroethylene & 2.0 & $\mathrm{U}$ & 2.0 & 2.1 & $\mathrm{U}$ & 2.1 & 2.1 & $\mathrm{U}$ & 2.1 & 2.1 & $\mathrm{U}$ & 2.1 & 2.0 & $\mathrm{U}$ & 2.0 \\
\hline Dichlorodiphenyltrichloroethane & 2.0 & $\mathrm{U}$ & 2.0 & 2.1 & $\mathrm{U}$ & 2.1 & 2.1 & $\mathrm{U}$ & 2.1 & 2.1 & $\mathrm{U}$ & 2.1 & 2.0 & $\mathrm{U}$ & 2.0 \\
\hline Dieldrin & 2.0 & $\mathrm{U}$ & 2.0 & 2.1 & $\mathrm{U}$ & 2.1 & 2.1 & $\mathrm{U}$ & 2.1 & 3.1 & $\mathrm{~J}$ & 2.1 & 2.0 & $\mathrm{U}$ & 2.0 \\
\hline Endosulfan I & 2.0 & $\mathrm{U}$ & 2.0 & 2.1 & $\mathrm{U}$ & 2.1 & 2.1 & $\mathrm{U}$ & 2.1 & 2.1 & $\mathrm{U}$ & 2.1 & 2.0 & $\mathrm{U}$ & 2.0 \\
\hline Endosulfan II & 2.0 & $\mathrm{U}$ & 2.0 & 2.1 & $\mathrm{U}$ & 2.1 & 2.1 & $\mathrm{U}$ & 2.1 & 2.1 & $\mathrm{U}$ & 2.1 & 2.0 & $\mathrm{U}$ & 2.0 \\
\hline Endosulfan sulfate & 2.0 & $\mathrm{U}$ & 2.0 & 2.1 & $\mathrm{U}$ & 2.1 & 2.1 & $\mathrm{U}$ & 2.1 & 2.1 & $\mathrm{U}$ & 2.1 & 2.0 & $\mathrm{U}$ & 2.0 \\
\hline Endrin & 2.0 & $\mathrm{U}$ & 2.0 & 2.1 & $\mathrm{U}$ & 2.1 & 2.1 & $\mathrm{U}$ & 2.1 & 2.1 & $\mathrm{U}$ & 2.1 & 2.0 & $\mathrm{U}$ & 2.0 \\
\hline Endrin aldehyde & 2.0 & $\mathrm{U}$ & 2.0 & 2.1 & $\mathrm{U}$ & 2.1 & 2.1 & $\mathrm{U}$ & 2.1 & 2.1 & $\mathrm{U}$ & 2.1 & 2.0 & $\mathrm{U}$ & 2.0 \\
\hline Endrin ketone & 2.0 & $\mathrm{U}$ & 2.0 & 2.1 & $\mathrm{U}$ & 2.1 & 2.1 & $\mathrm{U}$ & 2.1 & 2.1 & $\mathrm{U}$ & 2.1 & 2.0 & $\mathrm{U}$ & 2.0 \\
\hline Gamma-BHC (Lindane) & 2.0 & $\mathrm{U}$ & 2.0 & 2.1 & $\mathrm{U}$ & 2.1 & 2.1 & $\mathrm{U}$ & 2.1 & 2.1 & $\mathrm{U}$ & 2.1 & 2.0 & $\mathrm{U}$ & 2.0 \\
\hline gamma-Chlordane & 2.0 & $\mathrm{U}$ & 2.0 & 2.1 & $\mathrm{U}$ & 2.1 & 2.1 & $\mathrm{U}$ & 2.1 & 89 & & 2.1 & 2.0 & $\mathrm{U}$ & 2.0 \\
\hline Heptachlor & 2.0 & $\mathrm{U}$ & 2.0 & 2.1 & $\mathrm{U}$ & 2.1 & 2.1 & $\mathrm{U}$ & 2.1 & 25 & & 2.1 & 2.0 & $\mathrm{U}$ & 2.0 \\
\hline Heptachlor epoxide & 2.0 & $\mathrm{U}$ & 2.0 & 2.1 & $\mathrm{U}$ & 2.1 & 2.1 & $\mathrm{U}$ & 2.1 & 2.1 & $\mathrm{U}$ & 2.1 & 2.0 & $\mathrm{U}$ & 2.0 \\
\hline Methoxychlor & 2.0 & $\mathrm{U}$ & 2.0 & 2.1 & $\mathrm{U}$ & 2.1 & 2.1 & $\mathrm{U}$ & 2.1 & 2.1 & $\mathrm{U}$ & 2.1 & 2.0 & $\mathrm{U}$ & 2.0 \\
\hline Toxaphene & 20 & $\mathrm{U}$ & 20 & 21 & $\mathrm{U}$ & 21 & 21 & $\mathrm{U}$ & 21 & 21 & $\mathrm{U}$ & 21 & 20 & $\mathrm{U}$ & 20 \\
\hline
\end{tabular}


Table A-4. 120-F-1 Northwest Excavation Pre-Verification Sampling Results. (2 Pages)

\begin{tabular}{|c|c|c|c|c|c|c|c|c|c|c|c|c|c|c|c|}
\hline \multirow[t]{2}{*}{ Constituent } & \multicolumn{3}{|c|}{$\begin{array}{c}\text { J16B52 } \\
\text { Sample Location NW-6 } \\
\text { Sample Date } 2 / 19 / 08\end{array}$} & \multicolumn{3}{|c|}{$\begin{array}{c}\text { J16B53 } \\
\text { Sample Location NW-7 } \\
\text { Sample Date } 2 / 19 / 08\end{array}$} & \multicolumn{3}{|c|}{$\begin{array}{c}\text { J16B54 } \\
\text { Sample Location NW-8 } \\
\text { Sample Date } 2 / 19 / 08\end{array}$} & \multicolumn{3}{|c|}{$\begin{array}{c}\text { J16B55 } \\
\text { Sample Location NW-9 } \\
\text { Sample Date } 2 / 19 / 08\end{array}$} & \multicolumn{3}{|c|}{$\begin{array}{c}\text { J16B56 } \\
\text { Sample Location NW- } \\
10 \\
\text { Sample Date 2/19/08 }\end{array}$} \\
\hline & $\mu \mathrm{g} / \mathrm{kg}$ & $Q$ & PQL & $\mu \mathrm{g} / \mathrm{kg}$ & $Q$ & PQL & $\mu \mathrm{g} / \mathrm{kg}$ & $Q$ & PQL & $\mu \mathrm{g} / \mathrm{kg}$ & $\mathbf{Q}$ & PQL & $\mu \mathrm{g} / \mathrm{kg}$ & $\mathbf{Q}$ & PQL \\
\hline \multicolumn{16}{|c|}{ Pesticides } \\
\hline Aldrin & 2.0 & $\mathrm{U}$ & 2.0 & 2.2 & $\mathrm{U}$ & 2.2 & 2.0 & $\mathrm{U}$ & 2.0 & 2.1 & $\mathrm{U}$ & 2.1 & 2.1 & U & 2.1 \\
\hline Alpha-BHC & 2.0 & $U$ & 2.0 & 2.2 & $\mathrm{U}$ & 2.2 & 2.0 & $\mathrm{U}$ & 2.0 & 2.1 & $\mathrm{U}$ & 2.1 & 2.1 & $\mathrm{U}$ & 2.1 \\
\hline Alpha-Chlordane & 33 & & 2.0 & 2.2 & $\mathrm{U}$ & 2.2 & 2.0 & $\mathrm{U}$ & 2.0 & 8.4 & $\mathrm{~J}$ & 2.1 & 2.1 & $\mathrm{U}$ & 2.1 \\
\hline Beta-BHC & 2.0 & $\mathrm{U}$ & 2.0 & 2.2 & $\mathrm{U}$ & 2.2 & 2.0 & $\mathrm{U}$ & 2.0 & 2.1 & $\mathrm{U}$ & 2.1 & 2.1 & $\mathrm{U}$ & 2.1 \\
\hline Delta-BHC & 2.0 & $\mathrm{U}$ & 2.0 & 2.2 & $\mathrm{U}$ & 2.2 & 2.0 & $\mathrm{U}$ & 2.0 & 2.1 & $\mathrm{U}$ & 2.1 & 2.1 & $\mathrm{U}$ & 2.1 \\
\hline Dichlorodiphenyldichloroethane & 2.0 & $\mathrm{U}$ & 2.0 & 2.2 & $\mathrm{U}$ & 2.2 & 2.0 & $\mathrm{U}$ & 2.0 & 2.1 & $\mathrm{U}$ & 2.1 & 2.1 & $\mathrm{U}$ & 2.1 \\
\hline Dichlorodiphenyldichloroethylene & 2.0 & $\mathrm{U}$ & 2.0 & 2.2 & $\mathrm{U}$ & 2.2 & 2.0 & $\mathrm{U}$ & 2.0 & 2.1 & $\mathrm{U}$ & 2.1 & 2.1 & $\mathrm{U}$ & 2.1 \\
\hline Dichlorodiphenyltrichloroethane & 2.0 & $\mathrm{U}$ & 2.0 & 2.2 & $\mathrm{U}$ & 2.2 & 2.0 & $\mathrm{U}$ & 2.0 & 2.1 & $\mathrm{U}$ & 2.1 & 2.1 & $\mathrm{U}$ & 2.1 \\
\hline Dieldrin & 2.0 & $\mathrm{U}$ & 2.0 & 2.2 & $\mathrm{U}$ & 2.2 & 2.0 & $\mathrm{U}$ & 2.0 & 2.1 & $\mathrm{U}$ & 2.1 & 2.1 & $\mathrm{U}$ & 2.1 \\
\hline Endosulfan I & 2.0 & $\mathrm{U}$ & 2.0 & 2.2 & $\mathrm{U}$ & 2.2 & 2.0 & $\mathrm{U}$ & 2.0 & 2.1 & $\mathrm{U}$ & 2.1 & 2.1 & $\mathrm{U}$ & 2.1 \\
\hline Endosulfan II & 2.0 & $\mathrm{U}$ & 2.0 & 2.2 & $\mathrm{U}$ & 2.2 & 2.0 & $\mathrm{U}$ & 2.0 & 2.1 & $\mathrm{U}$ & 2.1 & 2.1 & $\mathrm{U}$ & 2.1 \\
\hline Endosulfan sulfate & 2.0 & $\mathrm{U}$ & 2.0 & 2.2 & $\mathrm{U}$ & 2.2 & 2.0 & $\mathrm{U}$ & 2.0 & 2.1 & $\mathrm{U}$ & 2.1 & 2.1 & $\mathrm{U}$ & 2.1 \\
\hline Endrin & 2.0 & $\mathrm{U}$ & 2.0 & 2.2 & $\mathrm{U}$ & 2.2 & 2.0 & $\mathrm{U}$ & 2.0 & 2.1 & $\mathrm{U}$ & 2.1 & 2.1 & $\mathrm{U}$ & 2.1 \\
\hline Endrin aldehyde & 2.0 & $\mathrm{U}$ & 2.0 & 2.2 & $\mathrm{U}$ & 2.2 & 2.0 & $\mathrm{U}$ & 2.0 & 2.1 & $\mathrm{U}$ & 2.1 & 2.1 & $\mathrm{U}$ & 2.1 \\
\hline Endrin ketone & 2.0 & $\mathrm{U}$ & 2.0 & 2.2 & $\mathrm{U}$ & 2.2 & 2.0 & $\mathrm{U}$ & 2.0 & 2.1 & $\bar{U}$ & 2.1 & 2.1 & $\mathrm{U}$ & 2.1 \\
\hline Gamma-BHC (Lindane) & 2.0 & $\mathrm{U}$ & 2.0 & 2.2 & $\mathrm{U}$ & 2.2 & 2.0 & $\mathrm{U}$ & 2.0 & 2.1 & $\mathrm{U}$ & 2.1 & 2.1 & U & 2.1 \\
\hline gamma-Chlordane & 39 & & 2.0 & 2.2 & $\mathrm{U}$ & 2.2 & 2.0 & $\mathrm{U}$ & 2.0 & 7.4 & $\mathrm{~J}$ & 2.1 & 2.1 & $\mathrm{U}$ & 2.1 \\
\hline Heptachlor & 16 & & 2.0 & 2.2 & $\mathrm{U}$ & 2.2 & 2.0 & $\bar{U}$ & 2.0 & 2.1 & $\mathrm{U}$ & 2.1 & 2.1 & $\mathrm{U}$ & 2.1 \\
\hline Heptachlor epoxide & 17 & & 2.0 & 2.2 & $\mathrm{U}$ & 2.2 & 2.0 & $\mathrm{U}$ & 2.0 & 2.1 & $\mathrm{U}$ & 2.1 & 2.1 & $\mathrm{U}$ & 2.1 \\
\hline Methoxychlor & 2.0 & $\mathrm{U}$ & 2.0 & 2.2 & $\mathrm{U}$ & 2.2 & 2.0 & $\mathrm{U}$ & 2.0 & 2.1 & $\mathrm{U}$ & 2.1 & 2.1 & $\mathrm{U}$ & 2.1 \\
\hline Toxaphene & 20 & $\mathrm{U}$ & 20 & 22 & $\mathrm{U}$ & 22 & 20 & $\mathrm{U}$ & 20 & 21 & $\mathrm{U}$ & 21 & 21 & $\mathrm{U}$ & 21 \\
\hline
\end{tabular}


Table A-5. 120-F-1 Northwest Excavation Pre-Verification Sampling Results. (2 Pages)

\begin{tabular}{|c|c|c|c|c|c|c|c|c|c|c|c|c|c|c|c|}
\hline \multirow[t]{2}{*}{ Constituent } & \multicolumn{3}{|c|}{\begin{tabular}{|c} 
J16DD4 \\
Sample Location NW-1 \\
Sample Date 3/5/08
\end{tabular}} & \multicolumn{3}{|c|}{$\begin{array}{c}\text { J16DD5 } \\
\text { Sample Location NW-2 } \\
\text { Sample Date 3/5/08 }\end{array}$} & \multicolumn{3}{|c|}{$\begin{array}{c}\text { J16DD6 } \\
\text { Sample Location NW-3 } \\
\text { Sample Date 3/5/08 }\end{array}$} & \multicolumn{3}{|c|}{$\begin{array}{c}\text { J16DD7 } \\
\text { Sample Location NW-4 } \\
\text { Sample Date 3/5/08 }\end{array}$} & \multicolumn{3}{|c|}{$\begin{array}{c}\text { J16DD8 } \\
\text { Sample Location NW-5 } \\
\text { Sample Date 3/5/08 }\end{array}$} \\
\hline & $\mu \mathrm{g} / \mathrm{kg}$ & $Q$ & PQL & $\mu \mathrm{g} / \mathrm{kg}$ & $Q$ & PQL & $\mu \mathrm{g} / \mathrm{kg}$ & $Q$ & PQL & $\mu \mathrm{g} / \mathrm{kg}$ & $Q$ & PQL & $\mu \mathrm{g} / \mathrm{kg}$ & $Q$ & PQL \\
\hline \multicolumn{16}{|c|}{ Pesticides } \\
\hline Aldrin & 1.3 & $\mathrm{U}$ & 1.3 & 1.3 & $\mathrm{U}$ & 1.3 & 1.3 & $\mathrm{U}$ & 1.3 & 1.3 & $\mathrm{U}$ & 1.3 & 1.3 & $\mathrm{U}$ & 1.3 \\
\hline Alpha-BHC & 1.3 & $\mathrm{U}$ & 1.3 & 1.3 & $\mathrm{U}$ & 1.3 & 1.3 & $\mathrm{U}$ & 1.3 & 1.3 & $\mathrm{U}$ & 1.3 & 1.3 & $\mathrm{U}$ & 1.3 \\
\hline Alpha-Chlordane & 1.3 & $\mathrm{U}$ & 1.3 & 4.2 & $\mathrm{~J}$ & 1.3 & 1.3 & $\mathrm{U}$ & 1.3 & 1.3 & $\mathrm{U}$ & 1.3 & 160 & & 1.3 \\
\hline Beta-BHC & 1.3 & $\mathrm{U}$ & 1.3 & 1.3 & $\mathrm{U}$ & 1.3 & 1.3 & \begin{tabular}{|l|}
$U$ \\
\end{tabular} & 1.3 & 1.3 & $\mathrm{U}$ & 1.3 & 1.3 & $\mathrm{U}$ & 1.3 \\
\hline Delta-BHC & 1.3 & $\mathrm{U}$ & 1.3 & 1.3 & $\mathrm{U}$ & 1.3 & 1.3 & $\mathrm{U}$ & 1.3 & 1.3 & $\mathrm{U}$ & 1.3 & 1.3 & $\mathrm{U}$ & 1.3 \\
\hline Dichlorodiphenyldichloroethane & 1.3 & $\mathrm{U}$ & 1.3 & 1.3 & $\mathrm{U}$ & 1.3 & 1.3 & $\mathrm{U}$ & 1.3 & 1.3 & $\mathrm{U}$ & 1.3 & 1.3 & $\mathrm{U}$ & 1.3 \\
\hline Dichlorodiphenyldichloroethylene & 1.3 & $\mathrm{U}$ & 1.3 & 1.3 & $\mathrm{U}$ & 1.3 & 1.3 & $\mathrm{U}$ & 1.3 & 1.3 & $\mathrm{U}$ & 1.3 & 1.3 & $\mathrm{U}$ & 1.3 \\
\hline Dichlorodiphenyltrichloroethane & 1.3 & $\mathrm{U}$ & 1.3 & 1.3 & $\mathrm{U}$ & 1.3 & 1.3 & \begin{tabular}{|l|l}
$U$ \\
\end{tabular} & 1.3 & 1.3 & $\mathrm{U}$ & 1.3 & 1.3 & $\mathrm{U}$ & 1.3 \\
\hline Dieldrin & 1.3 & $\mathrm{U}$ & 1.3 & 1.3 & $\mathrm{U}$ & 1.3 & 1.3 & $\mathrm{U}$ & 1.3 & 1.3 & $\mathrm{U}$ & 1.3 & 11 & & 1.3 \\
\hline Endosulfan I & 1.3 & $\mathrm{U}$ & 1.3 & 1.3 & $\mathrm{U}$ & 1.3 & 1.3 & $\mathrm{U}$ & 1.3 & 1.3 & $\mathrm{U}$ & 1.3 & 1.7 & $\mathbf{J}$ & 1.3 \\
\hline \begin{tabular}{|l|} 
Endosulfan II \\
\end{tabular} & 1.3 & $\mathrm{U}$ & 1.3 & 1.3 & $\mathrm{U}$ & 1.3 & 1.3 & $\mathrm{U}$ & 1.3 & 1.3 & $\mathrm{U}$ & 1.3 & 1.3 & $\mathrm{U}$ & 1.3 \\
\hline Endosulfan sulfate & 1.3 & $\mathrm{U}$ & 1.3 & 1.3 & $\mathrm{U}$ & 1.3 & 1.3 & $\mathrm{U}$ & 1.3 & 1.3 & $\mathrm{U}$ & 1.3 & 1.3 & $\mathrm{U}$ & 1.3 \\
\hline Endrin & 1.3 & $\mathrm{U}$ & 1.3 & 1.3 & $\mathrm{U}$ & 1.3 & 1.3 & $\mathrm{U}$ & 1.3 & 1.3 & $\bar{U}$ & 1.3 & 1.3 & $\mathrm{U}$ & 1.3 \\
\hline Endrin aldehyde & 1.3 & $\mathrm{U}$ & 1.3 & 1.3 & $\mathrm{U}$ & 1.3 & 1.3 & $\mathrm{U}$ & 1.3 & 1.3 & $\mathrm{U}$ & 1.3 & 1.3 & $\mathrm{U}$ & 1.3 \\
\hline \begin{tabular}{|l} 
Endrin ketone \\
\end{tabular} & 1.3 & $\mathrm{U}$ & 1.3 & 1.3 & $\mathrm{U}$ & 1.3 & 1.3 & \begin{tabular}{|l|}
$\mathrm{U}$ \\
\end{tabular} & 1.3 & 1.3 & $\mathrm{U}$ & 1.3 & 1.3 & $\mathrm{U}$ & 1.3 \\
\hline Gamma-BHC (Lindane) & 1.3 & $\mathrm{U}$ & 1.3 & 1.3 & $\mathrm{U}$ & 1.3 & 1.3 & $\mathrm{U}$ & 1.3 & 1.3 & $\mathrm{U}$ & 1.3 & 1.3 & $\mathrm{U}$ & 1.3 \\
\hline gamma-Chlordane & 1.3 & $\mathrm{U}$ & 1.3 & 4.6 & $\mathrm{~J}$ & 1.3 & 1.3 & $\mathrm{U}$ & 1.3 & 1.3 & $\mathrm{U}$ & 1.3 & 180 & & 1.3 \\
\hline Heptachlor & 1.3 & $\mathrm{U}$ & 1.3 & 1.3 & $\mathrm{U}$ & 1.3 & 1.3 & $\mathrm{U}$ & 1.3 & 1.3 & $\mathrm{U}$ & 1.3 & 65 & & 1.3 \\
\hline Heptachlor epoxide & 1.3 & $\mathrm{U}$ & 1.3 & 1.3 & $\mathrm{U}$ & 1.3 & 1.3 & $\mathrm{U}$ & 1.3 & 1.3 & $\mathrm{U}$ & 1.3 & 1.3 & $\mathrm{U}$ & 1.3 \\
\hline \begin{tabular}{|l} 
Methoxychlor \\
\end{tabular} & 1.3 & $\mathrm{U}$ & 1.3 & 1.3 & $\mathrm{U}$ & 1.3 & 1.3 & $\mathrm{U}$ & 1.3 & 1.3 & $\mathrm{U}$ & 1.3 & 1.3 & $\mathrm{U}$ & 1.3 \\
\hline Toxaphene & 13 & $\mathrm{U}$ & 13 & 13 & $\mathrm{U}$ & 13 & 13 & $\mathrm{U}$ & 13 & 13 & $\mathrm{U}$ & 13 & 13 & $\mathrm{U}$ & 13 \\
\hline
\end{tabular}


Table A-5. 120-F-1 Northwest Excavation Pre-Verification Sampling Results. (2 Pages)

\begin{tabular}{|c|c|c|c|c|c|c|c|c|c|c|c|c|c|c|c|}
\hline \multirow[t]{2}{*}{ Constituent } & \multicolumn{3}{|c|}{$\begin{array}{c}\text { J16DD9 } \\
\text { Sample Location NW-6 } \\
\text { Sample Date 3/5/08 }\end{array}$} & \multicolumn{3}{|c|}{$\begin{array}{c}\text { J16DF0 } \\
\text { Sample Location NW-7 } \\
\text { Sample Date 3/5/08 }\end{array}$} & \multicolumn{3}{|c|}{$\begin{array}{c}\text { J16DF1 } \\
\text { Sample Location NW-8 } \\
\text { Sample Date 3/5/08 }\end{array}$} & \multicolumn{3}{|c|}{$\begin{array}{c}\text { J16DF2 } \\
\text { Sample Location NW-9 } \\
\text { Sample Date 3/5/08 }\end{array}$} & \multicolumn{3}{|c|}{$\begin{array}{c}\text { J16DF3 } \\
\text { Sample Location NW- } \\
10 \\
\text { Sample Date } 3 / 5 / 08\end{array}$} \\
\hline & $\mu \mathrm{g} / \mathrm{kg}$ & $Q$ & PQL & $\mu \mathrm{g} / \mathrm{kg}$ & $Q$ & PQL & $\mu \mathrm{g} / \mathrm{kg}$ & $\mathbf{Q}$ & $\mathrm{PQL}$ & $\mu \mathrm{g} / \mathrm{kg}$ & $Q$ & PQL & $\mu \mathrm{g} / \mathrm{kg}$ & $\mathbf{Q}$ & PQL \\
\hline \multicolumn{16}{|c|}{ Pesticides } \\
\hline Aldrin & 1.3 & $\mathrm{U}$ & 1.3 & 1.3 & $\mathrm{U}$ & 1.3 & 1.3 & $\mathrm{U}$ & 1.3 & 1.3 & $\mathrm{U}$ & 1.3 & 1.3 & $\mathrm{U}$ & 1.3 \\
\hline Alpha-BHC & 1.3 & $\mathrm{U}$ & 1.3 & 1.3 & $\mathrm{U}$ & 1.3 & 1.3 & $\mathrm{U}$ & 1.3 & 1.3 & $\mathrm{U}$ & 1.3 & 1.3 & $\mathrm{U}$ & 1.3 \\
\hline Alpha-Chlordane & 1.3 & $\mathrm{U}$ & 1.3 & 1.3 & $\mathrm{U}$ & 1.3 & 1.3 & $\mathrm{U}$ & 1.3 & 1.3 & $\mathrm{U}$ & 1.3 & 1.3 & $\mathrm{U}$ & 1.3 \\
\hline Beta-BHC & 1.3 & $\mathrm{U}$ & 1.3 & 1.3 & $\mathrm{U}$ & 1.3 & 1.3 & $\mathrm{U}$ & 1.3 & 1.3 & $\mathrm{U}$ & 1.3 & 1.3 & $\mathrm{U}$ & 1.3 \\
\hline Delta-BHC & 1.3 & $\mathrm{U}$ & 1.3 & 1.3 & $\mathrm{U}$ & 1.3 & 1.3 & $\mathrm{U}$ & 1.3 & 1.3 & $\mathrm{U}$ & 1.3 & 1.3 & $\mathrm{U}$ & 1.3 \\
\hline Dichlorodiphenyldichloroethane & 1.3 & $\mathrm{U}$ & 1.3 & 1.3 & $\mathrm{U}$ & 1.3 & 1.3 & $\mathrm{U}$ & 1.3 & 1.3 & $\mathrm{U}$ & 1.3 & 1.3 & $\mathrm{U}$ & 1.3 \\
\hline Dichlorodiphenyldichloroethylene & 1.3 & $\mathrm{U}$ & 1.3 & 1.3 & $\mathrm{U}$ & 1.3 & 1.3 & $\mathrm{U}$ & 1.3 & 1.3 & $\mathrm{U}$ & 1.3 & 1.3 & $\mathrm{U}$ & 1.3 \\
\hline Dichlorodiphenyltrichloroethane & 1.3 & $\mathrm{U}$ & 1.3 & 1.3 & $\mathrm{U}$ & 1.3 & 1.3 & $\mathrm{U}$ & 1.3 & 1.3 & $\mathrm{U}$ & 1.3 & 1.3 & $\mathrm{U}$ & 1.3 \\
\hline Dieldrin & 1.3 & $\mathrm{U}$ & 1.3 & 1.3 & $\mathrm{U}$ & 1.3 & 1.3 & $\bar{U}$ & 1.3 & 1.3 & $\mathrm{U}$ & 1.3 & 1.3 & $\mathrm{U}$ & 1.3 \\
\hline \begin{tabular}{|l|} 
Endosulfan I \\
\end{tabular} & 1.3 & $\mathrm{U}$ & 1.3 & 1.3 & $\mathrm{U}$ & 1.3 & 1.3 & $\mathrm{U}$ & 1.3 & 1.3 & $\mathrm{U}$ & 1.3 & 1.3 & $\mathrm{U}$ & 1.3 \\
\hline Endosulfan II & 1.3 & $\mathrm{U}$ & 1.3 & 1.3 & $\mathrm{U}$ & 1.3 & 1.3 & $\mathrm{U}$ & 1.3 & 1.3 & $\mathrm{U}$ & 1.3 & 1.3 & $\mathrm{U}$ & 1.3 \\
\hline Endosulfan sulfate & 1.3 & $\mathrm{U}$ & 1.3 & 1.3 & $\mathrm{U}$ & 1.3 & 1.3 & $\mathrm{U}$ & 1.3 & 1.3 & $\mathrm{U}$ & 1.3 & 1.3 & $\mathrm{U}$ & 1.3 \\
\hline Endrin & 1.3 & $\mathrm{U}$ & 1.3 & 1.3 & $\mathrm{U}$ & 1.3 & 1.3 & $\mathrm{U}$ & 1.3 & 1.3 & $\mathrm{U}$ & 1.3 & 1.3 & $\mathrm{U}$ & 1.3 \\
\hline Endrin aldehyde & 1.3 & $\mathrm{U}$ & 1.3 & 1.3 & $\mathrm{U}$ & 1.3 & 1.3 & $\mathrm{U}$ & 1.3 & 1.3 & $\mathrm{U}$ & 1.3 & 1.3 & $\mathrm{U}$ & 1.3 \\
\hline Endrin ketone & 1.3 & $\mathrm{U}$ & 1.3 & 1.3 & $\mathrm{U}$ & 1.3 & 1.3 & $\mathrm{U}$ & 1.3 & 1.3 & $\mathrm{U}$ & 1.3 & 1.3 & $\mathrm{U}$ & 1.3 \\
\hline Gamma-BHC (Lindane) & 1.3 & $\mathrm{U}$ & 1.3 & 1.3 & $\mathrm{U}$ & 1.3 & 1.3 & $\mathrm{U}$ & 1.3 & 1.3 & $\mathrm{U}$ & 1.3 & 1.3 & $\mathrm{U}$ & 1.3 \\
\hline gamma-Chlordane & 1.3 & $\mathrm{U}$ & 1.3 & 1.3 & $\mathrm{U}$ & 1.3 & 1.3 & $\mathrm{U}$ & 1.3 & 1.3 & $\mathrm{U}$ & 1.3 & 1.3 & $\mathrm{U}$ & 1.3 \\
\hline Heptachlor & 1.3 & $\mathrm{U}$ & 1.3 & 1.3 & $\mathrm{U}$ & 1.3 & 1.3 & $\mathrm{U}$ & 1.3 & 1.3 & $\mathrm{U}$ & 1.3 & 1.3 & $\mathrm{U}$ & 1.3 \\
\hline Heptachlor epoxide & 1.3 & $\mathrm{U}$ & 1.3 & 1.3 & $\mathrm{U}$ & 1.3 & 1.3 & $\mathrm{U}$ & 1.3 & 1.3 & $\mathrm{U}$ & 1.3 & 1.3 & $\mathrm{U}$ & 1.3 \\
\hline Methoxychlor & 1.3 & $\mathrm{U}$ & 1.3 & 1.3 & $\mathrm{U}$ & 1.3 & 1.3 & $\mathrm{U}$ & 1.3 & 1.3 & $\mathrm{U}$ & 1.3 & 1.3 & $\mathrm{U}$ & 1.3 \\
\hline Toxaphene & 13 & $\mathrm{U}$ & 13 & 13 & $\mathrm{U}$ & 13 & 13 & $\mathrm{U}$ & 13 & 13 & $\mathrm{U}$ & 13 & 13 & $\mathrm{U}$ & 13 \\
\hline
\end{tabular}


Table A-6. 120-F-1 Northwest Excavation Pre-Verification Sampling Results.

\begin{tabular}{|c|c|c|c|c|}
\hline \multirow[t]{2}{*}{ Constituent } & \multicolumn{3}{|c|}{$\begin{array}{c}\text { J16DJ2 } \\
\text { Sample Location NW-5 } \\
\text { Sample Date 3/11/08 }\end{array}$} & \\
\hline & $\mu \mathrm{g} / \mathrm{kg}$ & Q & PQL & \\
\hline \multicolumn{4}{|c|}{ Pesticides } & \\
\hline Aldrin & 1.3 & $\mathrm{U}$ & 1.3 & \\
\hline Alpha-BHC & 1.3 & $\mathrm{U}$ & 1.3 & \\
\hline Alpha-Chlordane & 1.3 & $\mathrm{U}$ & 1.3 & \\
\hline Beta-BHC & 1.3 & $\mathrm{U}$ & 1.3 & \\
\hline Delta-BHC & 1.3 & $\mathrm{U}$ & 1.3 & \\
\hline Dichlorodiphenyldichloroethane & 1.3 & $\mathrm{U}$ & 1.3 & \\
\hline \begin{tabular}{|l|} 
Dichlorodiphenyldichloroethylene \\
\end{tabular} & 1.3 & $\mathrm{U}$ & 1.3 & \\
\hline Dichlorodiphenyltrichloroethane & 1.3 & $\mathrm{U}$ & 1.3 & \\
\hline Dieldrin & 1.3 & U & 1.3 & \\
\hline Endosulfan I & 1.3 & $\mathrm{U}$ & 1.3 & \\
\hline Endosulfan II & 1.3 & U & 1.3 & \\
\hline Endosulfan sulfate & 1.3 & $\mathrm{U}$ & 1.3 & \\
\hline Endrin & 1.3 & $\mathrm{U}$ & 1.3 & \\
\hline Endrin aldehyde & 1.3 & $\mathrm{U}$ & 1.3 & \\
\hline Endrin ketone & 1.3 & U & 1.3 & \\
\hline Gamma-BHC (Lindane) & 1.3 & $\mathrm{U}$ & 1.3 & \\
\hline gamma-Chlordane & 1.3 & $\mathrm{U}$ & 1.3 & \\
\hline Heptachlor & $\overline{1.3}$ & $\mathrm{U}$ & 1.3 & \\
\hline Heptachlor epoxide & 1.3 & $\mathrm{U}$ & 1.3 & \\
\hline Methoxychlor & 1.3 & $\mathrm{U}$ & 1.3 & \\
\hline Toxaphene & 13 & \begin{tabular}{|l|l}
$U$ \\
\end{tabular} & 13 & \\
\hline
\end{tabular}




\section{APPENDIX B}

PHOTOS FROM 120-F-1 GLASS DUMP WASTE SITE 


\section{0-F-1 Waste Site Excavation.}

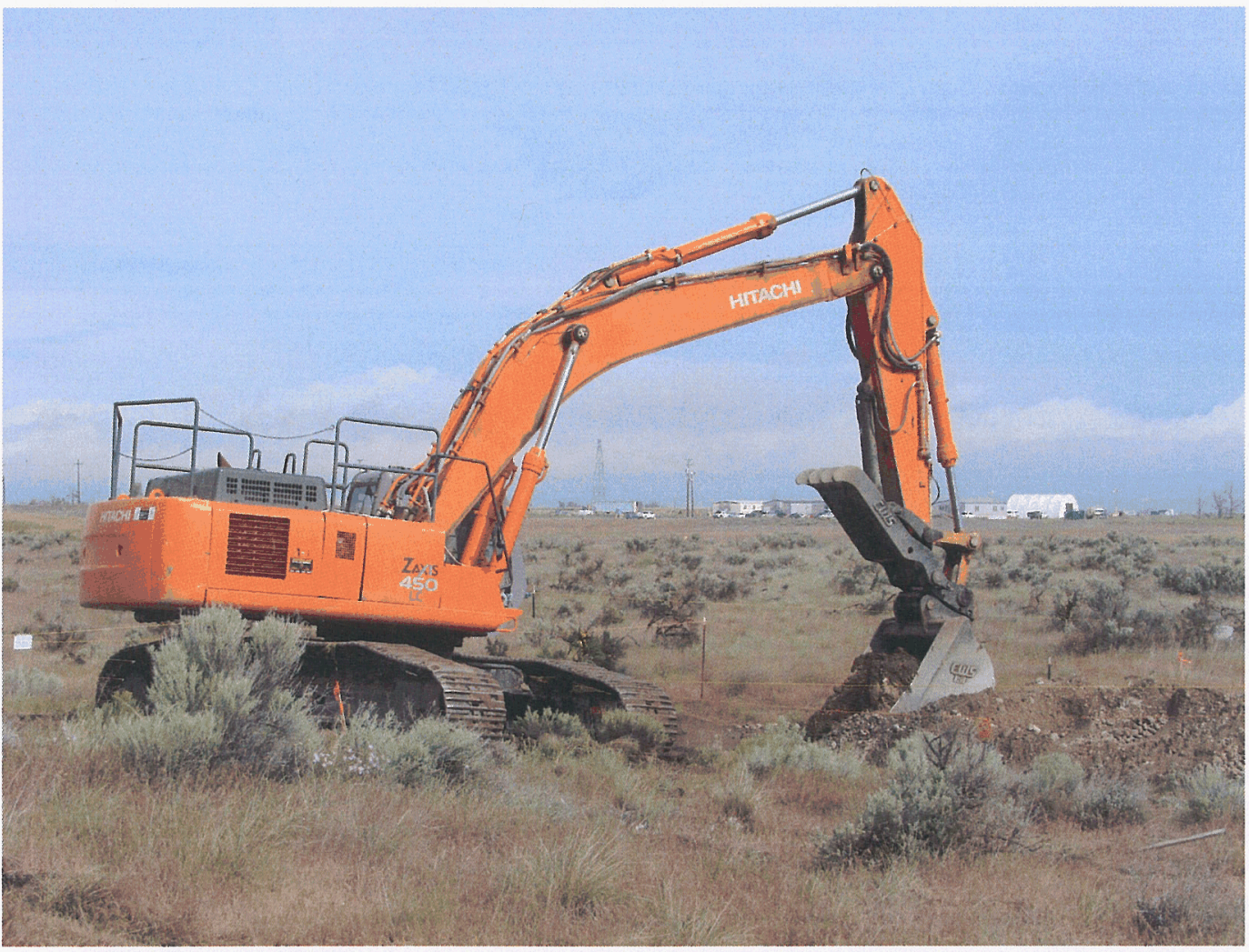

Laboratory Bottles, Incandescent Bulbs, and Fluorescent Bulbs From 120-F-1.

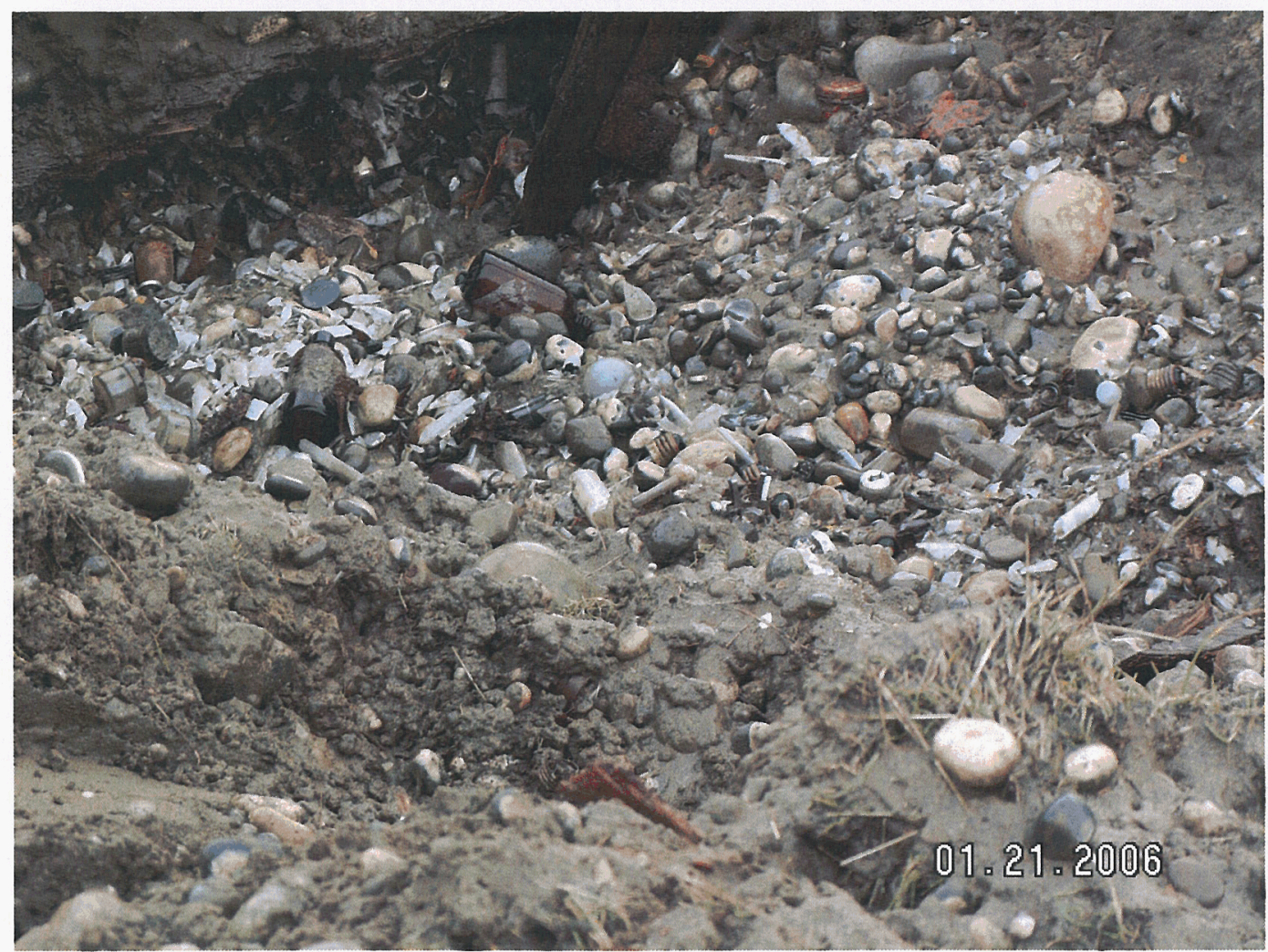


Laboratory Bottles, Incandescent Bulbs, and Batteries From 120-F-1.

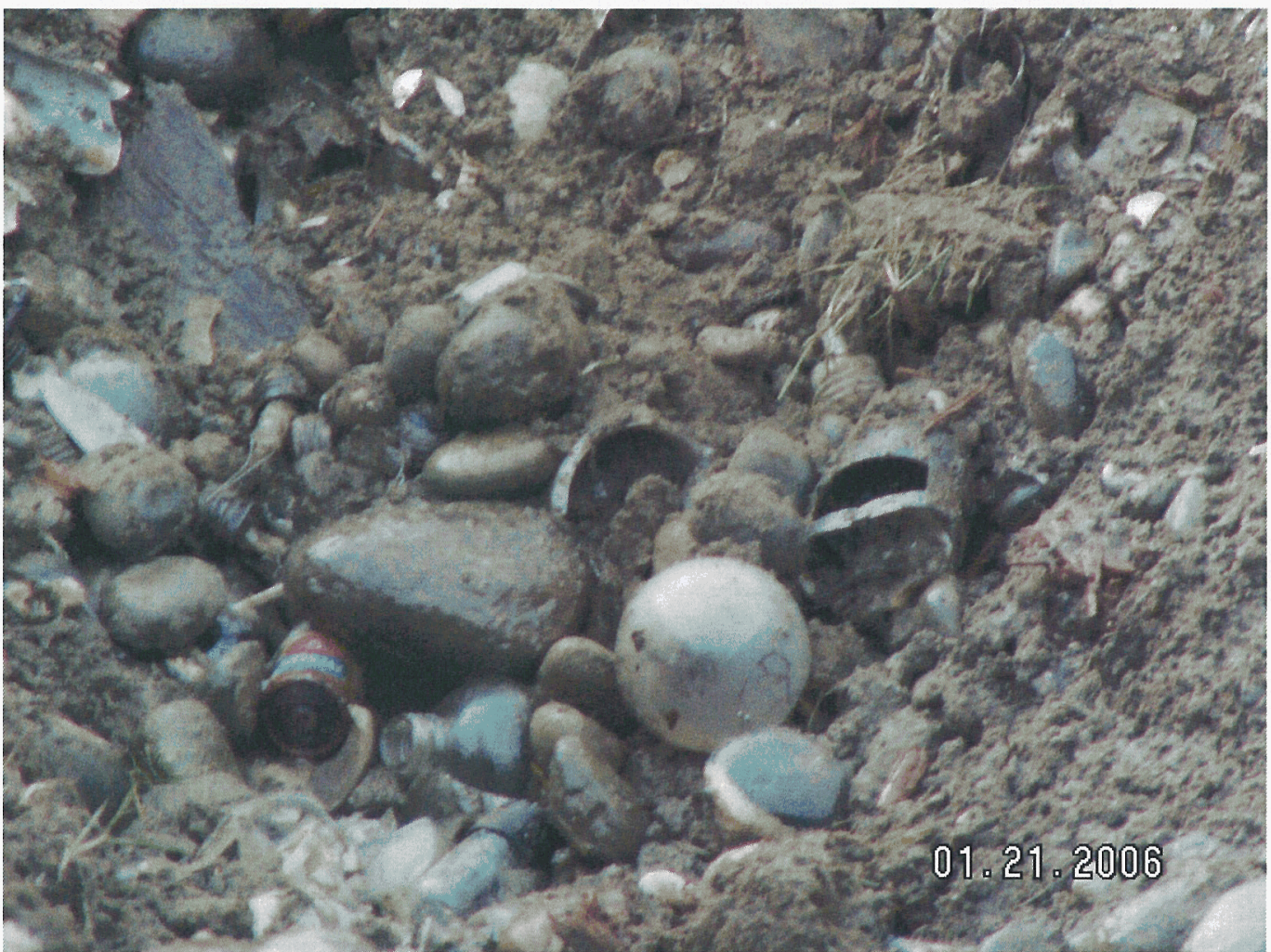

Metal Debris from 120-F-1.

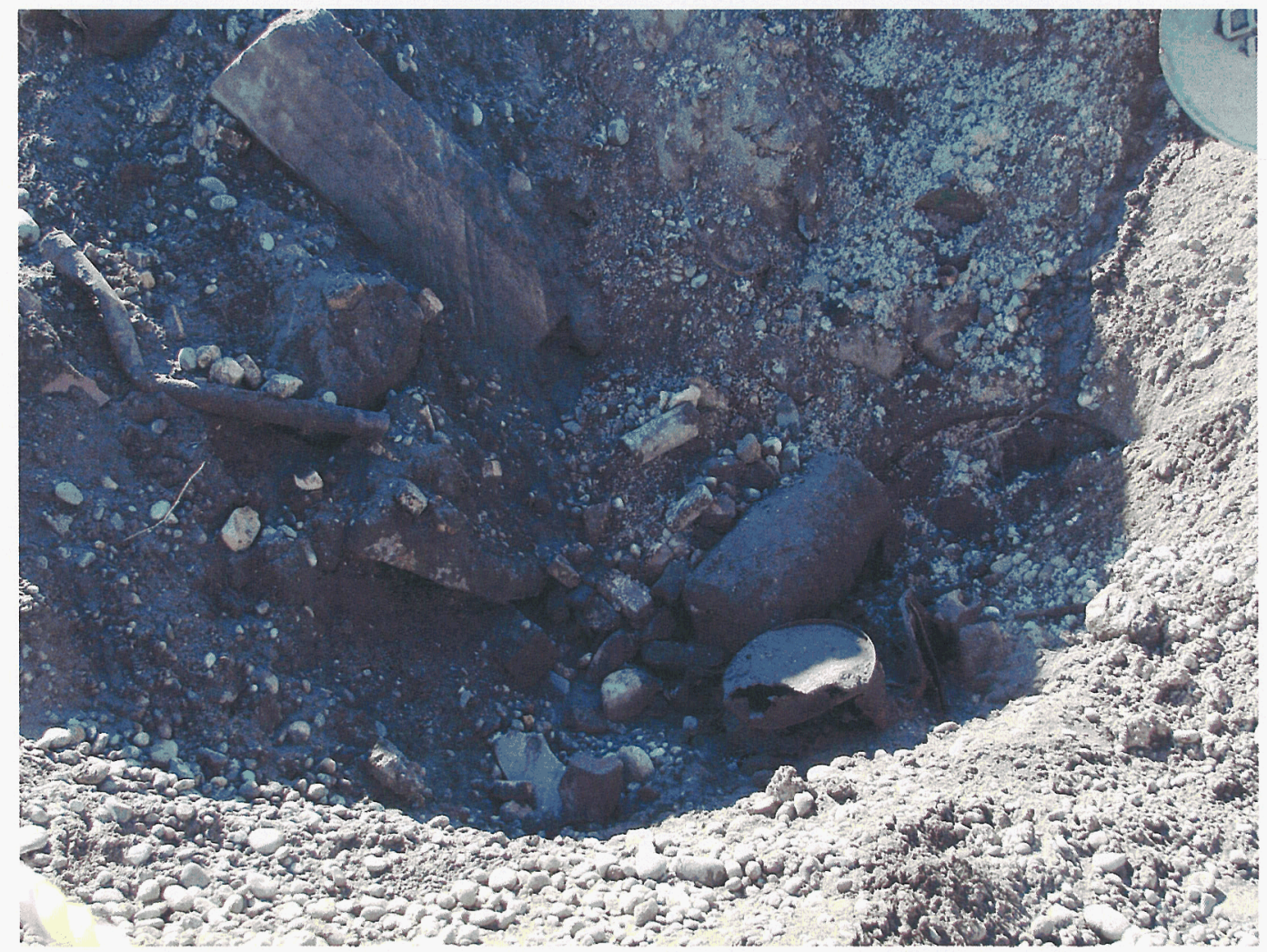


Yellow Stained Soil from 120-F-1 Southwest Excavation.

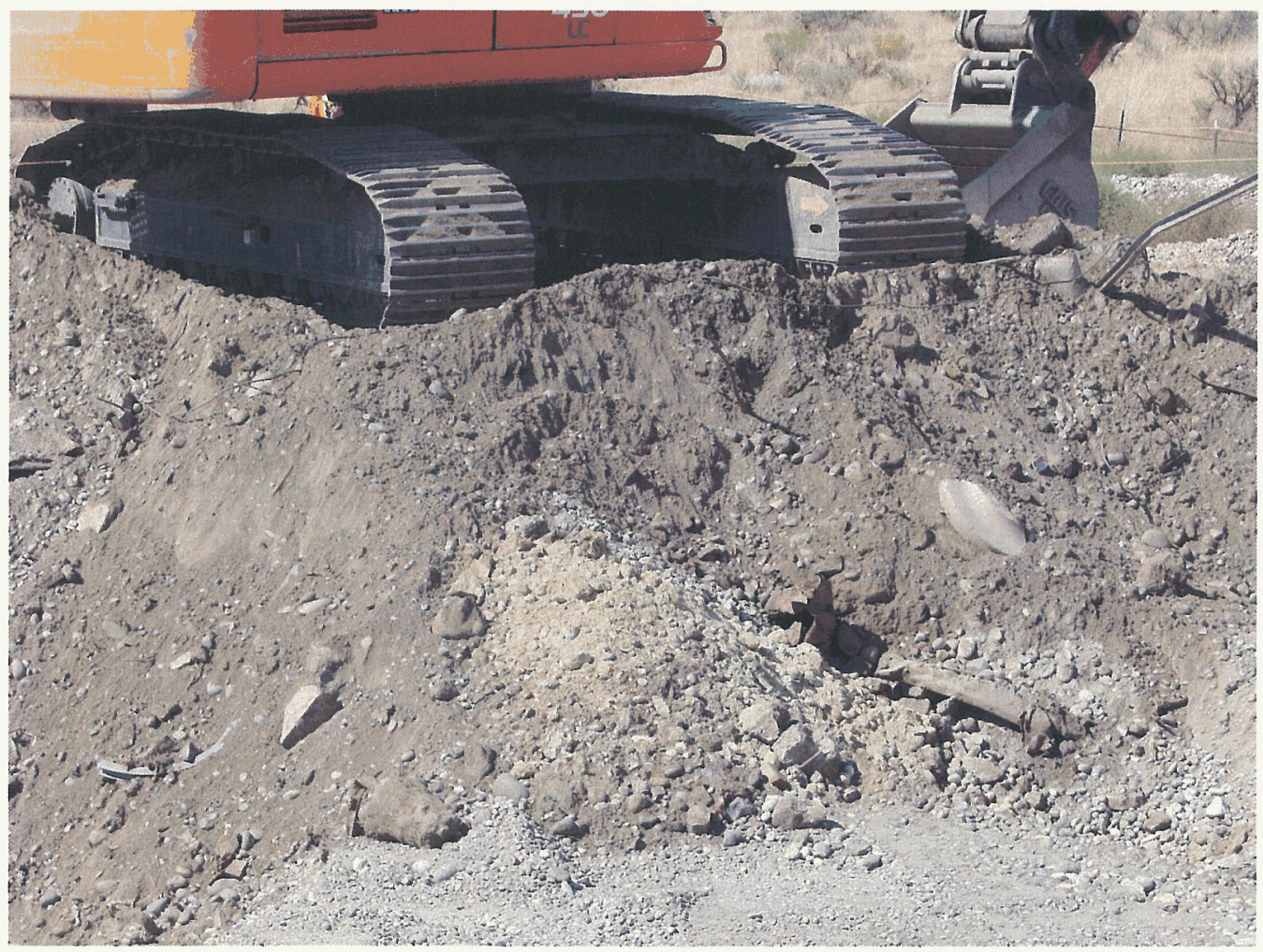

Breached, Oily Drum from 120-F-1.

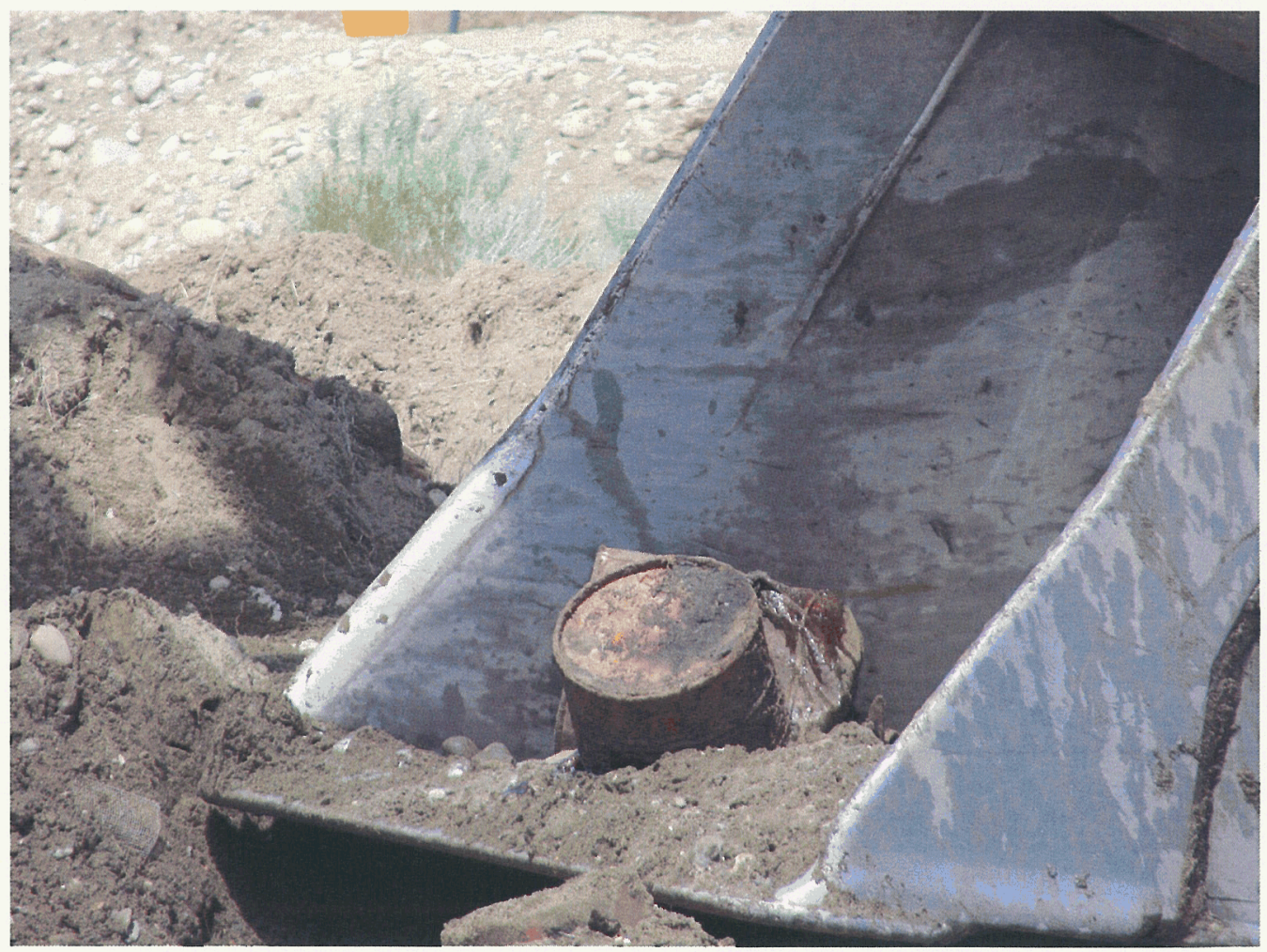




\section{APPENDIX C}

CALCULATION BRIEF 


\section{APPENDIX C}

\section{CALCULATIONS}

The calculations in this appendix are kept in the active Washington Closure Hanford project files and are available upon request. When the project is completed, the file will be stored in a U.S. Department of Energy, Richland Operations Office, repository. This calculation has been prepared in accordance with ENG-1, Engineering Services, ENG-1-4.5, "Project Calculation," Washington Closure Hanford, Richland, Washington. The following calculations are provided in this appendix:

120-F-1 Cleanup Verification 95\% UCL Calculation, 0100F-CA-V0350, Rev. 0

120-F-1 Waste Site Cleanup Verification Hazard Quotient and Carcinogenic Risk Calculation, 0100F-CA-V0355, Rev. 1

\section{DISCLAIMER FOR CALCULATIONS}

The calculations provided in this appendix have been generated to document compliance with established cleanup levels. These calculations should be used in conjunction with other relevant documents in the administrative record. 


\section{CALCULATION COVER SHEET}

Project Title: 100-F Field Remediation

Job No. 14655

Area: $100-\mathrm{F}$

Discipline: Environmental

${ }^{*}$ Calculation No: 0100F-CA-V03 50

Subject: 120-F-1 Cleanup Verification 95\% UCL Calculation

Computer Program: Excel

Program No: Excel 2003

The attached calculations have been generated to document compliance with established cleanup levels. These calculations should be used in conjunction with other relevant documents in the administrative record.

Committed Calculation $\nwarrow \quad$ Preliminary $\Gamma \quad$ Superseded $\Gamma \quad$ Voided $\Gamma$

\begin{tabular}{|c|c|c|c|c|c|c|}
\hline Rev. & Sheet Numbers & Originator & Checker & Reviewer & Approval & Date \\
\hline 0 & $\begin{array}{c}\text { Cover }=1 \\
\text { Sheets }=16 \\
\text { Attm. } 1=18 \\
\text { Total }=35\end{array}$ & H. M. Sulloway & L. D. Habel & NA & J. D. Fancher & \\
\hline & & & & & & \\
\hline & & & & & & \\
\hline & & & - & & & \\
\hline & & & & & & \\
\hline & & & & & & \\
\hline
\end{tabular}

SUMMARY OF REVISION

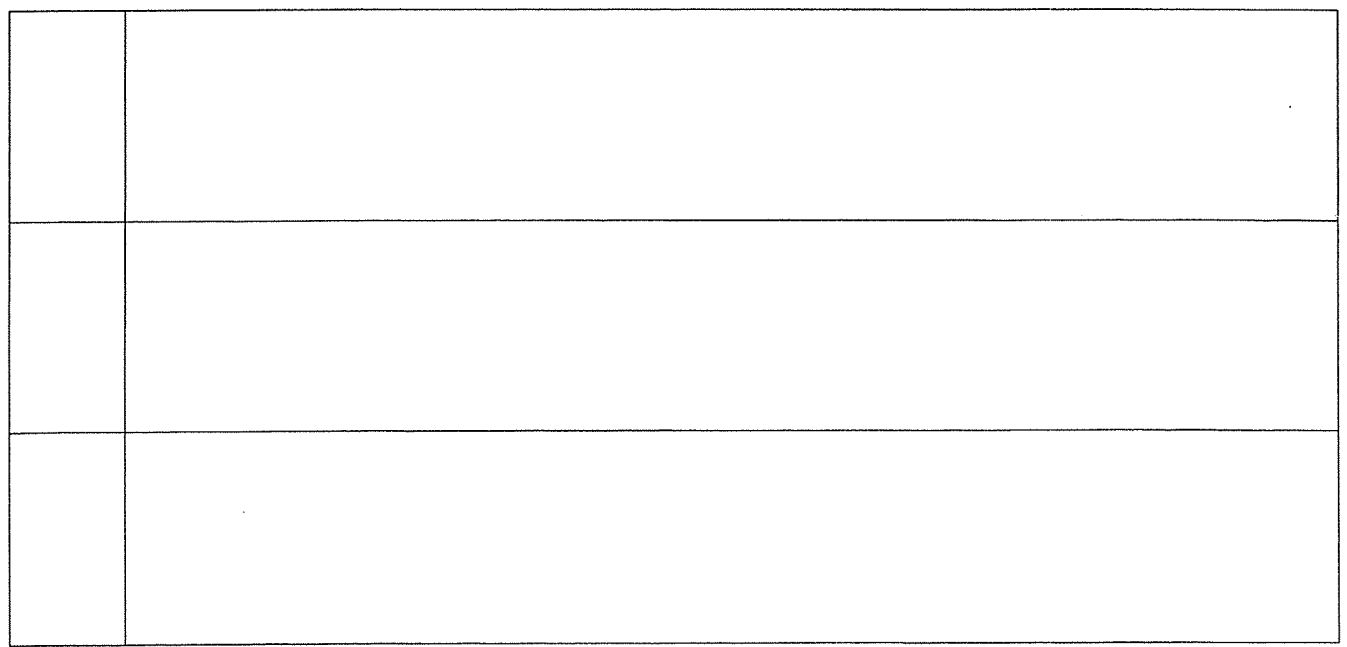




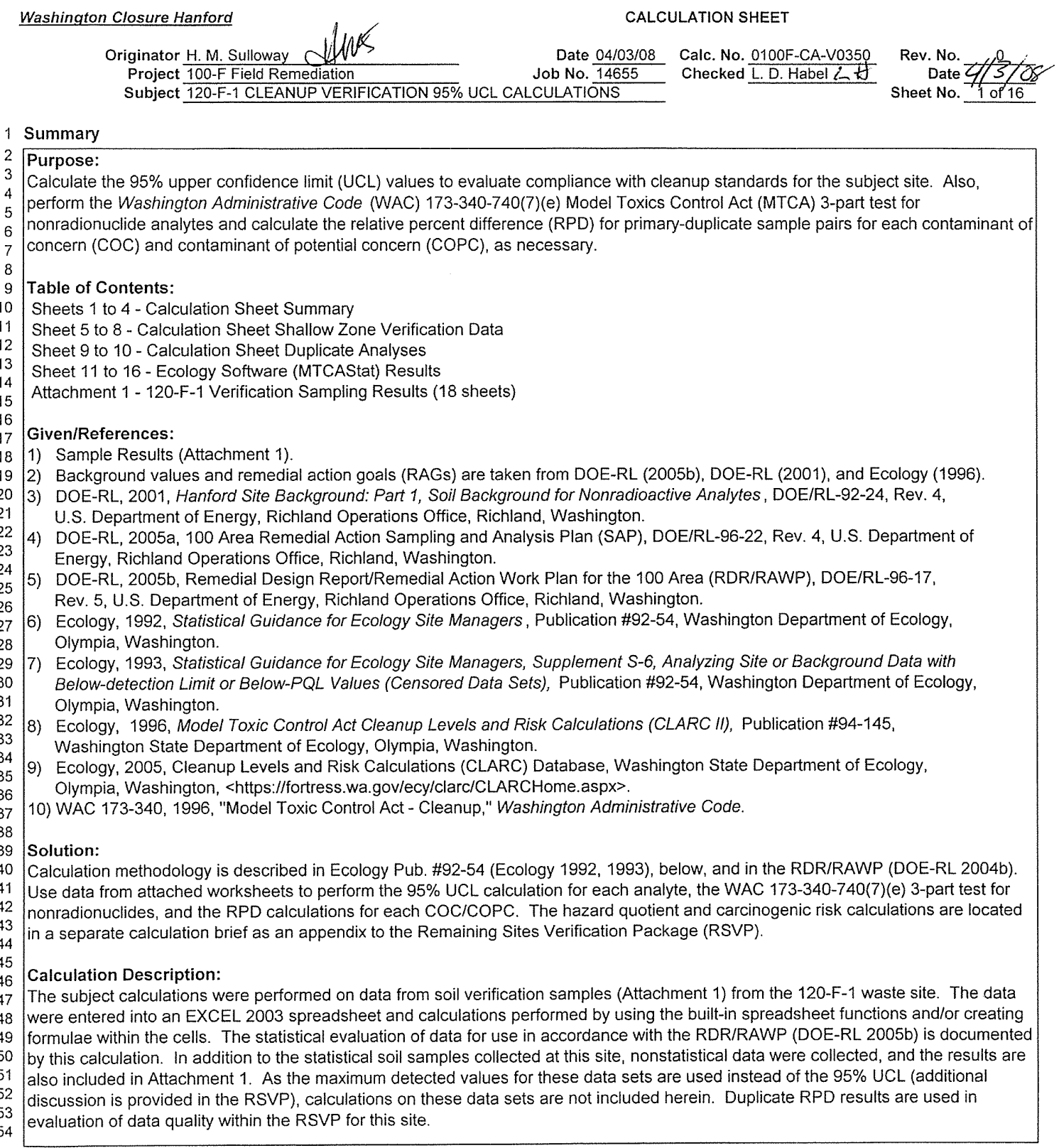


Washington Closure Hanford

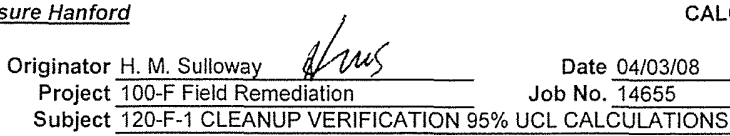

CALCULATION SHEET

\author{
Calc. No. 0100F-CA-V0350 \\ Checked L.D. Habel idl
}

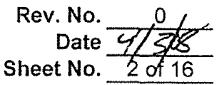

1 Summary (continued)

2 Methodology:

For nonradioactive analytes with $\leq 50 \%$ of the data below detection limits the statistical value calculated to evaluate the effectiveness of cleanup is the $95 \%$ UCL. For nonradioactive analytes with $>50 \%$ of the data below detection limits, as determined by direct inspection of the sample results (Attachment 1), the maximum detected value for the data set is used instead of the $95 \%$ UCL, and no further calculations are performed for those data sets. For convenience, these maximum detected values are included in the summary tables that follow. The $95 \%$ UCL was not calculated for data sets with no reported detections. Calculated cleanup levels are not available in Ecology (2005) under WAC 173-340-740(3) for aluminum, calcium, iron, magnesium, potassium, silicon, and sodium; therefore, these constituents are not considered site COCs/COPCs and are also not included in these calculations. The $95 \%$ UCL values were also not calculated for radium-226, radium-228, thorium-228, thorium-232, and potassium-40, as these isotopes are not related to the operational history of the site and thus not considered COCs/COPCs.

All nonradionuclide data reported as being undetected are set to $1 / 2$ the detection limit value for calculation of the statistics (Ecology 1993). For radionuclide data, calculation of the statistics was done on the reported value. In cases where the laboratory does not report a value below the minimal detectable activity (MDA), half of the MDA is used in the calculation. For the statistical evaluation of duplicate sample pairs, the samples are averaged before being included in the data set, after adjustments for censored data as described above.

For nonradionuclides, the WAC 173-340 statistical guidance suggests that a test for distributional form be performed on the data and the $95 \%$ UCL calculated on the appropriate distribution using Ecology software. For nonradionuclide small data sets ( $\mathrm{n}<10$ ) the calculations are performed assuming nonparametric distribution, so no tests for distribution are performed. For nonradionuclide data sets of ten or greater, as for the subject site, distributional testing is done using Ecology's MTCAStat software (Ecology 1993). Due to differences in addressing censored data between the RDR/RAWP (DOE-RL 2005b) and MTCAStat coding and due to a limitation in the MTCAStat coding (no direct capability to address variable quantitation limits within a data set), substitutions for censored data are performed before software input and the resulting data set treated as uncensored.

The WAC 173-340-740(7)(e) 3-part test is performed for nonradionuclide analytes only and determines if 1) the $95 \%$ UCL exceeds the most stringent cleanup limit for each $\mathrm{COPC} / \mathrm{COC}$

2) greater than $10 \%$ of the raw data exceed the most stringent cleanup limit for each COPC/COC,

3) the maximum value of the raw data set exceeds two times the most stringent cleanup limit for each COPC/COC.

The RPD is calculated when both the primary value and the duplicate value for a given analyte are above detection limits and are greater than 5 times the target detection limit (TDL). The TDL is a laboratory detection limit pre-determined for each analytical method and is listed in Table II-1 of the SAP (DOE-RL 2004a). Where direct evaluation of the attached sample data showed that a given analyte was not detected in the primary and/or duplicate sample, further evaluation of the RPD value was not performed. The RPD calculations use the following formula:

$$
\mathrm{RPD}=[|\mathrm{M}-\mathrm{S}| /((\mathrm{M}+\mathrm{S}) / 2)]^{*} 100
$$

$$
\text { where }, \quad M=\text { Main Sample Value } \quad S=\text { Split (or duplicate) Sample Value }
$$

For quality assurance/quality control (QAVQ) split and duplicate RPD calculations, a value less than $30 \%$ indicates the data compare favorably. For regulatory splits, a threshold of $35 \%$ is used (EPA 1994). If the RPD is greater than $30 \%$ (or $35 \%$ for regulatory split data), further investigation regarding the usability of the data is performed. No split samples were collected for cleanup verification of the subject site. Additional discussion is provided in the data quality assessment section of the applicable RSVP, as necessary. 









\begin{tabular}{|l|c|c|c|c|}
\hline \multicolumn{3}{|c|}{$\begin{array}{c}\text { Northwest Excavation Results Summary - Shallow Zone } \\
\text { Excavation }\end{array}$} \\
\hline \multicolumn{2}{|c|}{ Analyte } & $\begin{array}{c}95 \% \text { UCL } \\
\text { Result }\end{array}$ & $\begin{array}{c}\text { Maximum } \\
\text { Value }^{\mathrm{a}}\end{array}$ & Units \\
\hline
\end{tabular}

13 Chromium

14 Cobalt

15 Copper

16 Lead

17 Manganese

18 Nickel

19 Vanadium

20 Zinc

21 Sulfate

22 Bis(2-eth

23 Antimony

24 Hexavalent chromium

25 Selenium

26 Chloride

27 Nitrate

28 alpha-Chlordane

29 gamma-Chlordane

\begin{tabular}{l|r}
30 & Di-n-butylphthalate \\
\hline
\end{tabular}

$31{ }^{a}$ The $95 \%$ UCL result or maximum value, depending on data

32 censorship, as described in the methodology section.

Relative Percent Difference Results, J16DV6 and J16DV7 - QA/QC Analysis ${ }^{b}$

\begin{tabular}{|c|c|}
\hline Analyte & Duplicate Analysis \\
\hline Aluminum & $3.4 \%$ \\
\hline Barium & $4.5 \%$ \\
\hline Calcium & $2.8 \%$ \\
\hline Chromium & $9.0 \%$ \\
\hline Copper & $8.3 \%$ \\
\hline Iron & $3.9 \%$ \\
\hline Magnesium & $1.0 \%$ \\
\hline Manganese & $1.9 \%$ \\
\hline Silicon & $6.0 \%$ \\
\hline Vanadium & $2.2 \%$ \\
\hline Zinc & $1.4 \%$ \\
\hline Nitrate & $24.4 \%$ \\
\hline
\end{tabular}

${ }^{b}$ Relative percent difference evaluation was not required for analytes not included in this table. 
Washington Closure Hantord

Originator H. M. Sulloway Proiect 100.F Field Remediaticn

Datc
Job No.
J46355

Calc. No. $\frac{0100 F-\text { CA-V0os50 }}{\text { Checked }}$
L. D. Haoei LAt

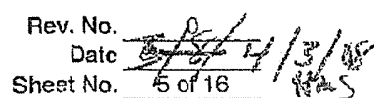

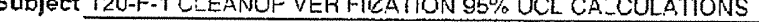





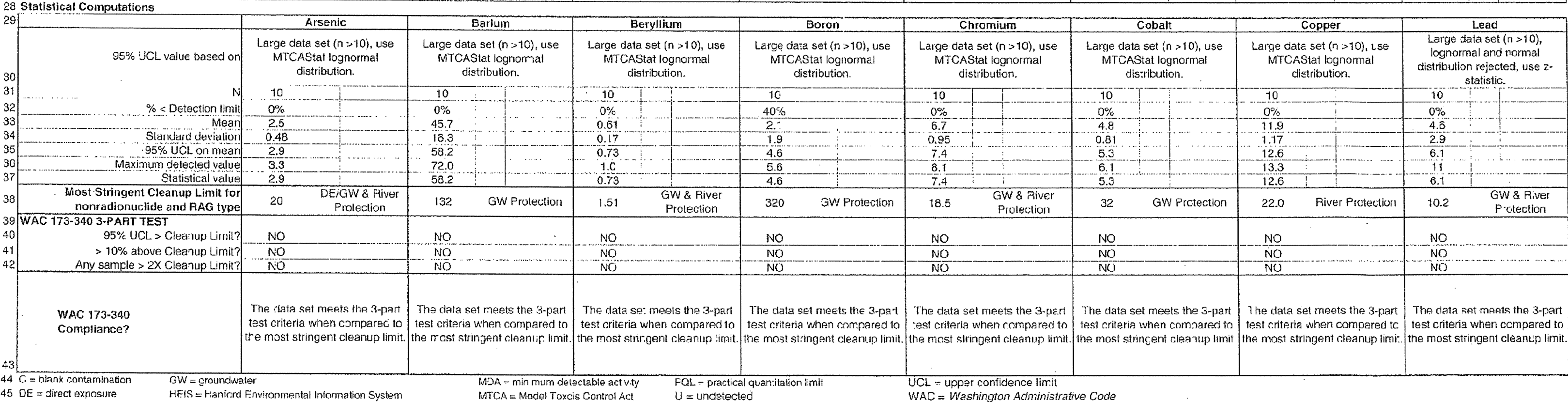




\section{Washington Closure Hanford}

Originator H. M. Sulloway $14 h \mathrm{~S}$

Subject 120-F-1 CLEANUP VERIFICATION 95\% UCL CALCULATIONS

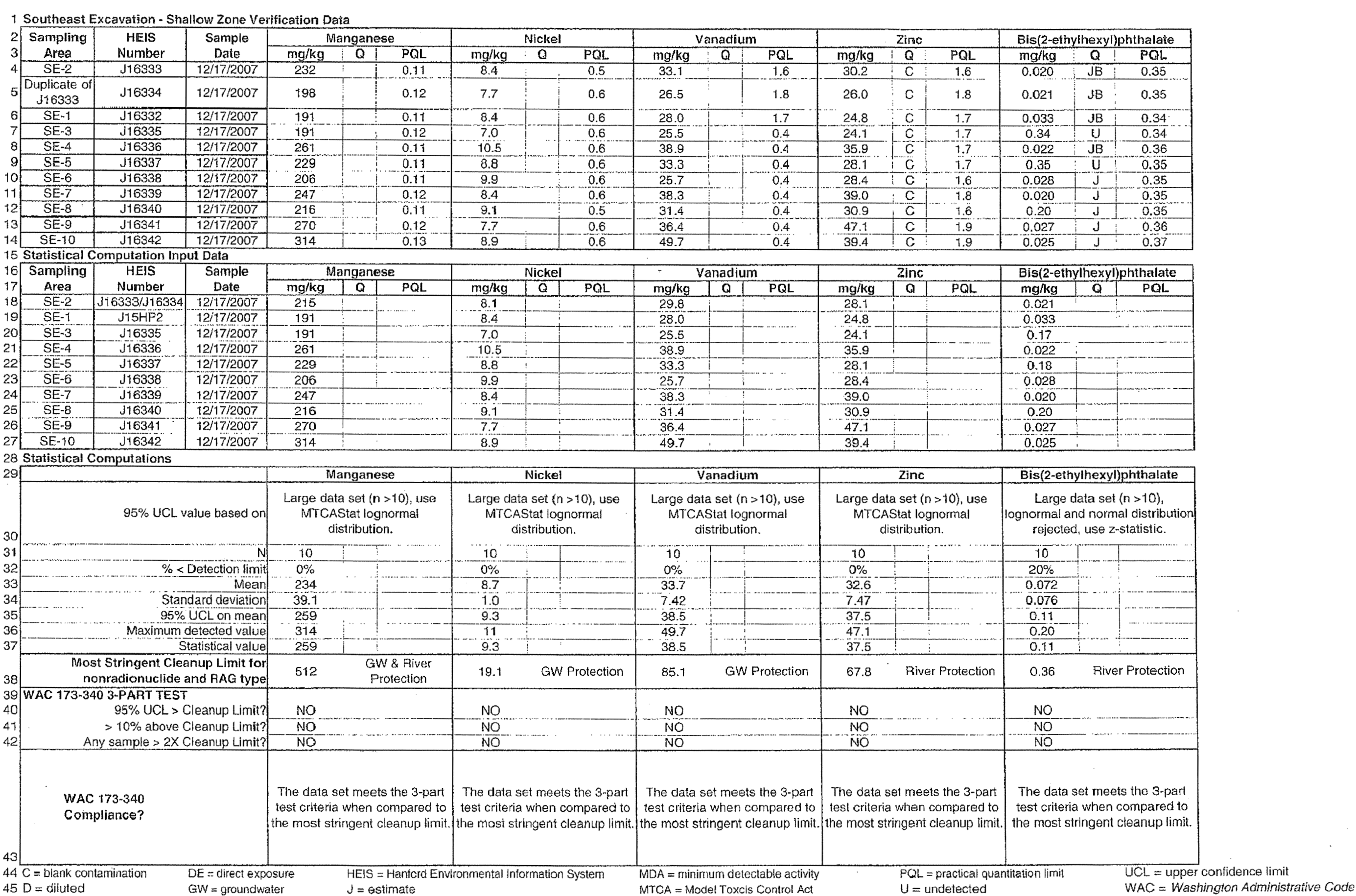


Washingion Closure Haniord

Originator H.M. Sulloway dich

$\begin{aligned} \text { Date } & 04 / 03 / 08 \\ \text { Job No. } & 14655\end{aligned}$

Calc. No. O100F-CA-Vo350
Checked L.D. Habel

Rev. No. $\begin{array}{ll}0 \\ \text { Date } \frac{410 \%}{70 \% 16}\end{array}$

Subject $\frac{100-\text { F Field Remediation }}{120-\text {-F-t CLLANUP VERIFICATION } 95 \% \text { UCL CALCULATIONS }}$

1 Noithwest Excavation - Shallow Zone Verification Data

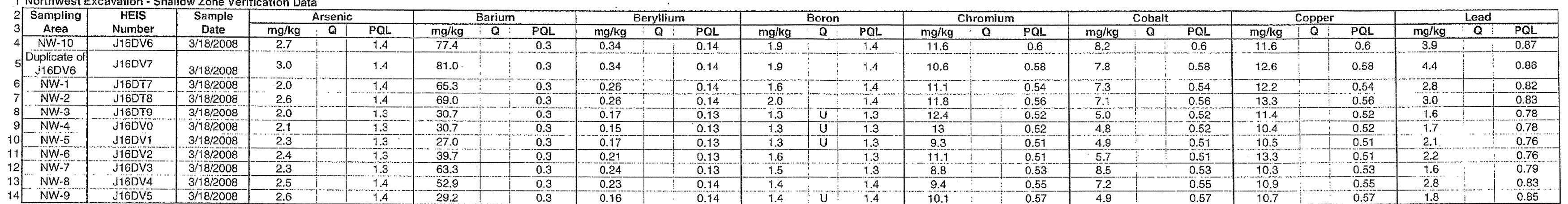

14
15
15 Statistical
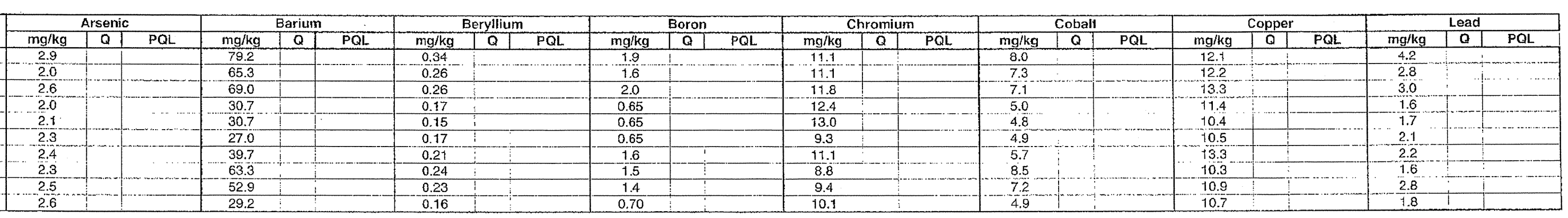






\section{Washington Closure Hanford}

Originator H.M. Sulloway HW H S

Date $\frac{04 / 03 / 08}{14655}$

Calc. No. $\frac{\text { 0100F-CA-V0350 }}{\text { Checked }}$
L. D. Habel LWL

Rev. No. $4 / 0 / 08$

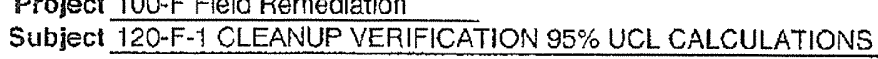

1 Norhwest Excavation - Shalliow Zone Verification Data





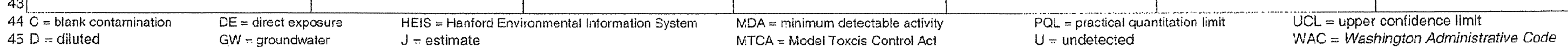


Washingion Closure Hanford

Originator H.M. Sulloway da two

Project
CALCULATION SHEET
\begin{aligned} & Date $04 / 03 / 08 \\ &$ Job No. 14655 \\ & \hline\end{aligned}
Calc. No. O100F-CA-V0350
Rev. No. $4 \mathbb{1}^{0}$
Sheer No. $\frac{9 / 5 / 08}{96}$

\begin{tabular}{|c|c|c|c|c|c|c|c|c|c|c|c|c|c|c|c|c|c|c|c|c|c|c|c|c|c|}
\hline \multirow{4}{*}{\begin{tabular}{c|c} 
& Sampling \\
3 & Area \\
$4 E-2$
\end{tabular}} & & & & & & & & & & & & & & & & & & & & & & & & & \\
\hline & \multirow{3}{*}{$\begin{array}{l}\text { Sample } \\
\text { Number } \\
\end{array}$} & \multirow{2}{*}{$\begin{array}{c}\text { Sample } \\
\text { Date }\end{array}$} & \multicolumn{3}{|c|}{ Aluminum } & \multicolumn{3}{|c|}{ Arsenic } & \multicolumn{3}{|c|}{ Barium } & \multicolumn{2}{|c|}{ Beryllium } & \multicolumn{3}{|c|}{ Boron } & \multicolumn{3}{|c|}{ Calcium } & \multicolumn{3}{|c|}{ Chromium } & \multicolumn{3}{|c|}{ Cobalt } \\
\hline & & & $\mathrm{mg} / \mathrm{kg}$ & & $\mathrm{POL}$ & $\mathrm{mg} / \mathrm{kg}$ & Ta & $\mathrm{PQL}$ & $\mathrm{mg} / \mathrm{kg}$ & al & $\mathrm{POL}$ & $\mathrm{mg} / \mathrm{kg}$ & \begin{tabular}{l|l}
$Q$ & $P Q L$ \\
\end{tabular} & $\mathrm{mg} / \mathrm{kg}$ & Q & PQL & $\mathrm{mg} / \mathrm{kg}$ & Q & $\mathrm{POL}$ & $\mathrm{mg} / \mathrm{kg}$ & $a$ & $\mathrm{PQL}$ & $\mathrm{mg} / \mathrm{kg}$ & Q & $P Q L$ \\
\hline & & $12 / 17 / 2007$ & 4960 & C & 10.5 & 1.8 & & 1.3 & 49.9 & c) & 0.26 & 0.56 & 0.13 & 1.7 & & 1.3 & 4920 & c & 10.5 & 6.0 & & 0.53 & 4.7 & & 0.53 \\
\hline $\begin{array}{c}\text { Duplicate of } \\
\mathrm{J} 16333\end{array}$ & & & & I & & & & 15 & & & & & & & & & & $n$ & & & & & & & \\
\hline \multicolumn{26}{|l|}{ Analysis: } \\
\hline \multicolumn{3}{|c|}{ TDL } & \multicolumn{3}{|c|}{5} & \multicolumn{3}{|c|}{10} & \multicolumn{3}{|c|}{2} & \multicolumn{2}{|r|}{0.5} & \multirow{2}{*}{\multicolumn{3}{|c|}{ (con }} & \multicolumn{3}{|c|}{100} & \multirow{2}{*}{\multicolumn{3}{|c|}{$\frac{1}{\text { Yes (continue) }}$}} & \multirow{2}{*}{\multicolumn{3}{|c|}{$\frac{2}{\text { Yes (continue) }}$}} \\
\hline \multirow{4}{*}{$\begin{array}{l}\text { Dupll } \\
\text { Anal }\end{array}$} & \multirow{2}{*}{\multicolumn{2}{|c|}{$\begin{array}{l}\text { Bcth }>\text { PQL? } \\
\text { Both >5xTDL? }\end{array}$}} & \multirow{2}{*}{\multicolumn{3}{|c|}{ Yes (continue) }} & \multirow{2}{*}{\multicolumn{3}{|c|}{ No-Stop (acceptable) }} & \multirow{2}{*}{\multicolumn{3}{|c|}{ Yes (continue) }} & \multirow{2}{*}{\multicolumn{2}{|c|}{ No-Stop (acceptable) }} & \multirow{2}{*}{\multicolumn{3}{|c|}{ No-Stop (acceptable) }} & \multirow{2}{*}{\multicolumn{3}{|c|}{ Yes (continue) }} & & & & & & \\
\hline & & & Yes & & RPD) & & & & & (calc) & & & & & & & & & & \multicolumn{3}{|c|}{ Yes (calc APD) } & \multicolumn{3}{|c|}{ No-Slop (acceptable } \\
\hline & & & & $8.6 \%$ & & & & & & $12.3 \%$ & & & & & & & & $2.6 \%$ & & & $1.7 \%$ & & & & \\
\hline & Differen & $>2$ TDL? & Not & appli & cable & No. & acce & ptable & & I applic & & No- & icceptable & No- -2 & accep & fable & & applic & & & & able & No- & - - acce & tiabie \\
\hline
\end{tabular}

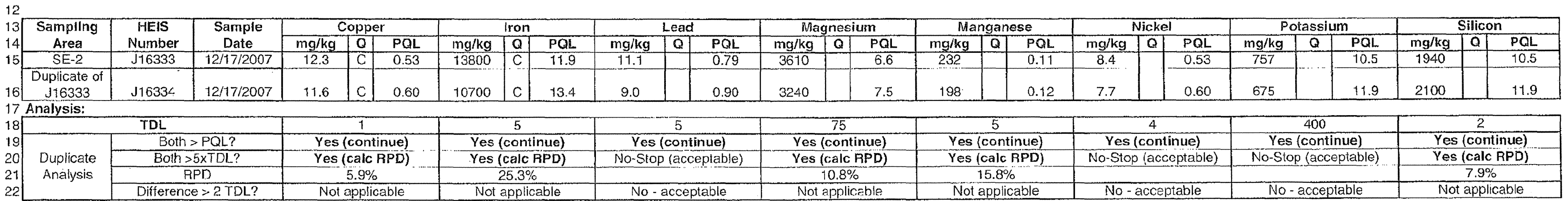

\begin{tabular}{|c|c|c|c|c|c|c|c|c|c|c|c|c|c|c|c|c|c|c|c|c|c|}
\hline & \multirow{3}{*}{$\begin{array}{c}\text { Sampling } \\
\text { Area }\end{array}$} & \multirow{2}{*}{$\begin{array}{c}\text { HEIS } \\
\text { Number } \\
\end{array}$} & \multirow{2}{*}{$\begin{array}{c}\text { Sample } \\
\text { Daie }\end{array}$} & \multicolumn{3}{|c|}{ Sodium } & \multicolumn{3}{|c|}{ Vanadium } & \multicolumn{3}{|c|}{ Zinc } & \multicolumn{3}{|c|}{ Chloride } & \multicolumn{3}{|c|}{ Sulfate } & \multicolumn{3}{|c|}{$\begin{array}{l}\text { Bis (2-ethylhexyi) } \\
\text { phithalate }\end{array}$} \\
\hline & & & & $\mathrm{mg} / \mathrm{kg}$ & Q & $\mathrm{POL}$ & $\mathrm{mg} / \mathrm{kg}$ & Q & $\mathrm{PQL}$ & $\mathrm{mg} / \mathrm{kg}$ & a & $\mathrm{PQL}$ & $\mathrm{mg} / \mathrm{kg}$ & a & PQL & $\mathrm{mg} / \mathrm{kg}$ & a & $\mathrm{PQL}$ & & Q & \\
\hline & & $\mathrm{J} 16333$ & $12 / 17 / 2007$ & 200 & $C$ & 5.3 & 33.1 & & 0.37 & 30.2 & c & 1.6 & 3.1 & & 2.4 & 493 & D & 24 & 0.021 & $J B$ & 0.35 \\
\hline & $\begin{array}{l}\text { Duplicate of } \\
\mathrm{J16333}\end{array}$ & 334 & 07 & 200 & 10 & 6.0 & 26.5 & & 0.42 & & c & 1.8 & 3.4 & & 2.3 & 183 & D & 23 & 0.34 & u & 0.34 \\
\hline \multirow{2}{*}{\multicolumn{22}{|c|}{ nalysis: }} \\
\hline & \multicolumn{3}{|c|}{ TDL } & \multicolumn{3}{|c|}{50} & \multicolumn{3}{|c|}{2.5} & \multicolumn{3}{|c|}{1} & \multicolumn{3}{|c|}{2} & \multicolumn{3}{|c|}{5} & \multicolumn{3}{|c|}{0.33} \\
\hline & \multirow{4}{*}{$\begin{array}{l}\text { Duplcate } \\
\text { Analysis }\end{array}$} & \multirow{2}{*}{\multicolumn{2}{|c|}{$\frac{\text { Both }>\text { PQL? }}{\text { Both > 5 XTDL? }}$}} & \multirow{3}{*}{\multicolumn{3}{|c|}{$\begin{array}{c}\text { Yes (continue) } \\
\text { No-Stop (acceptable) }\end{array}$}} & \multirow{2}{*}{\multicolumn{3}{|c|}{$\begin{array}{l}\text { Yes (continue) } \\
\text { Yes (calc RPD) }\end{array}$}} & \multirow{2}{*}{\multicolumn{3}{|c|}{$\begin{array}{l}\text { Yes (continue) } \\
\text { Yes (calc RPD) }\end{array}$}} & & \multirow{2}{*}{\multicolumn{3}{|c|}{$\begin{array}{l}\text { Yes (continue) } \\
\text { Yes (calc RPD) }\end{array}$}} & \multicolumn{3}{|c|}{ No-Stop (acceptable) } \\
\hline & & & & & & & & & & & & & \multirow{2}{*}{\multicolumn{3}{|c|}{ No-Stop (acceptable) }} & & & & \multirow{3}{*}{\multicolumn{3}{|c|}{ - }} \\
\hline & & \multirow{2}{*}{\multicolumn{2}{|c|}{ RPD }} & & & & \multicolumn{3}{|c|}{$\frac{22.1 \%}{20}$} & \multicolumn{3}{|c|}{$\frac{\text { Yes (calc RPD) }}{14.9 \%}$} & & & & \multirow{2}{*}{\multicolumn{3}{|c|}{$91.7 \%$}} & & & \\
\hline & & & & No- - & acce & able & Not & applitic & able & Not & applic & & No- & acce & table & & appli & & & & table \\
\hline
\end{tabular}


Washington Closure Hanford

Originator H. M. Sulloway dh wws

Project 100-F Field Kemediation
CALCUL.ATION SHEET

$$
\begin{aligned}
& \begin{aligned}
\text { Date } & 04,03 / 08 \\
\text { Job No. } & 14655
\end{aligned} \\
& \text { Calc. No. 0100F-CA-V0350 } \\
& \text { Rev. No. } 0 \\
& \text { Date } \frac{\pi / 3 / 08}{10 \text { of } 16}
\end{aligned}
$$

\begin{tabular}{|c|c|c|c|c|c|c|c|c|c|c|c|c|c|c|c|c|c|c|c|c|c|}
\hline & \multirow{2}{*}{$\begin{array}{c}\text { Sampling } \\
\text { Area }\end{array}$} & \multirow{2}{*}{$\begin{array}{c}\text { HEIS } \\
\text { Number }\end{array}$} & \multirow{2}{*}{$\begin{array}{c}\text { Sample } \\
\text { Date }\end{array}$} & \multicolumn{3}{|c|}{ Sodium } & \multicolumn{3}{|c|}{ Vanadium } & \multicolumn{3}{|c|}{ Zinc } & \multicolumn{3}{|c|}{ Nitrate } & \multicolumn{3}{|c|}{ Sulfate } & \multicolumn{3}{|c|}{$\begin{array}{l}\text { Bis (2-ethylhexyl) } \\
\text { phthalate }\end{array}$} \\
\hline & & & & $\mathrm{mg} / \mathrm{kg}$ & 0 & $\mathrm{POL}$ & $\mathrm{mg} / \mathrm{kg}$ & Q & $\mathrm{PQL}$ & $\mathrm{mg} / \mathrm{kg}$ & 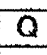 & $P Q L$ & $\mathrm{mg} / \mathrm{kg}$ & a & $\mathrm{PQL}$ & $\mathrm{mg} / \mathrm{kg}$ & $Q$ & $\mathrm{PQL}$ & $\mathrm{mg} / \mathrm{kg}$ & a & $\mathrm{POL}$ \\
\hline & $\frac{\text { NW-10 }}{\text { Duplicatio of }}$ & J16DVE & 3/1/28/2008 & & & 5.8 & & & & 42.5 & & & 25.3 & & 2.68 & & & 2.7 & 0.018 & 5 & 0.36 \\
\hline & J16DVE & $J 16 \mathrm{DV} 7$ & $3 / 18 / 2008$ & 126 & & 5.8 & 52.8 & & 0.4 & 41.9 & & 1.7 & 19.8 & & 2.64 & 4.3 & & 2.6 & 0.028 & |JB| & 0.36 \\
\hline \multicolumn{22}{|c|}{ nalysis: } \\
\hline & \multicolumn{3}{|c|}{ TDL } & \multicolumn{3}{|c|}{50} & \multicolumn{3}{|c|}{2.5} & \multicolumn{3}{|c|}{1} & \multicolumn{3}{|c|}{2.5} & \multicolumn{3}{|c|}{5} & \multicolumn{3}{|c|}{0.33} \\
\hline & \multirow{2}{*}{$\begin{array}{l}\text { Duplicate } \\
\text { Analysis }\end{array}$} & \multicolumn{2}{|c|}{$\begin{array}{l}\text { Both > PQL? } \\
\text { Both }>5 \times \text { TD } \text { ? }\end{array}$} & \multicolumn{3}{|c|}{$\begin{array}{c}\text { Yes (continue) } \\
\text { No-Stop (acceptable) }\end{array}$} & & Yes (continue) & & \multirow{2}{*}{\multicolumn{3}{|c|}{$1.4 \%$}} & \multirow{2}{*}{\multicolumn{3}{|c|}{$24.4 \%$}} & \multicolumn{3}{|c|}{ No-Stop (acceptable) } & \multicolumn{3}{|c|}{ No-Stop (a.cceptable) } \\
\hline $32+252$ & & \multicolumn{2}{|c|}{\begin{tabular}{|l} 
APD \\
Dillerence $>2 \mathrm{TDL}$ ?
\end{tabular}} & \multicolumn{3}{|c|}{ No - acceptable } & \multicolumn{3}{|c|}{$\frac{2.2 \%}{\text { Not apolicabie }}$} & & & & & & & \multicolumn{3}{|c|}{ No - acceptable } & \multicolumn{3}{|c|}{ No-acceptable } \\
\hline
\end{tabular}

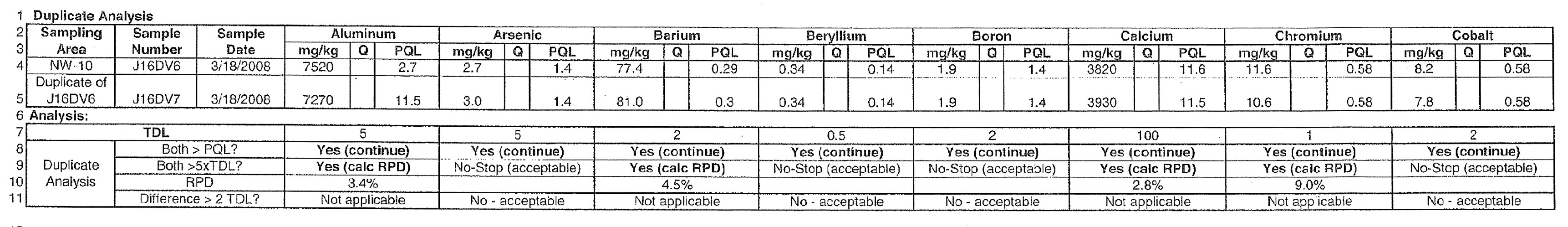

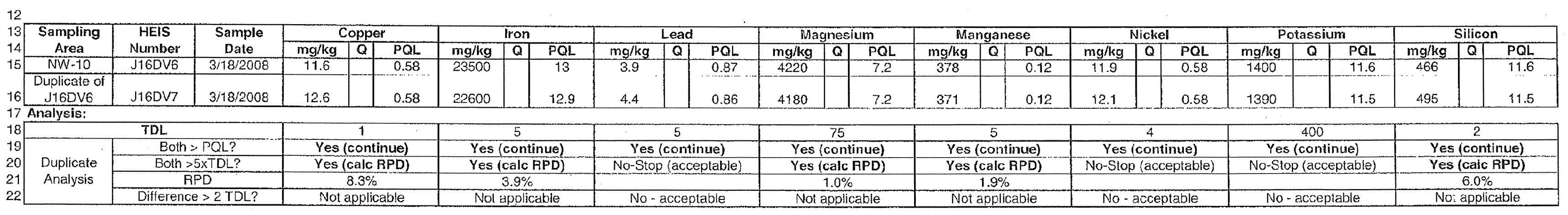




\section{Washington Closure Hanford}

Originator H.M. Sulloway dthe Subject 120 -F-F-1 CLEAMUUP VERIFICATION $95 \%$ UCL CALCULATIONS

1 Southeast Excavation

\begin{tabular}{|c|c|c|c|c|c|}
\hline Southeast & Excavation & & & & cology Soft \\
\hline DATA & ID & Arsenic $95 \%$ U & $\overline{L C z}$ & ulation & \\
\hline & & & & & \\
\hline & & Number of samples & & Uncensored values & \\
\hline $\begin{array}{l}2.5 \\
3.2\end{array}$ & 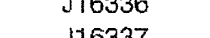 & Uncensosered & 10 & Mean & \\
\hline 2.3 & 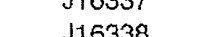 & Detection limil or POL & & Lognorir mean & in 2.5 \\
\hline 2.4 & J16339 & Method detection limit & & Median & 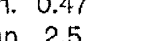 \\
\hline 2.4 & J16340 & TOTAL & 10 & Min. & $\begin{array}{lll}n & 17\end{array}$ \\
\hline 2.5 & J16341 & & & & x. 3.3 \\
\hline 2.8 & J16342 & & & & \\
\hline & & n? & & $\begin{array}{l}\text { Normal distribution? } \\
\text { rscouredis: }\end{array}$ & \\
\hline
\end{tabular}

Date $\frac{04 / 03 / 08}{14655}$

Calc. No. $\frac{0100 F-C A-V 0350}{L .2 \text {. Habel } 1-45}$
Checked

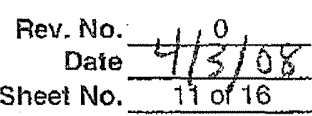

\begin{tabular}{|c|c|c|c|c|c|}
\hline \multirow{9}{*}{$\begin{array}{c}\text { DATA } \\
47.0 \\
28.8 \\
30.4 \\
54.7 \\
36.0 \\
36.3 \\
52.3 \\
29.3 \\
70.2 \\
72.0\end{array}$} & 10 & \multicolumn{3}{|c|}{ Barium $95 \%$ UCL. Calculation } & \\
\hline & J16335 & Number of samples & \multirow{4}{*}{10} & Uncersisored values & \\
\hline & Ј16336 & Uncensored & & & \\
\hline & J16337 & Censored & & Lognormal mean & 45.9 \\
\hline & $\begin{array}{l}316338 \\
J 1639\end{array}$ & Detection limit or $P Q L$ & & Sto devn. & 16.3 \\
\hline & $\begin{array}{l}J 16339 \\
\text { J16340 }\end{array}$ & $\begin{array}{l}\text { Method detection limit } \\
\text { TOTAL. }\end{array}$ & \multirow[t]{2}{*}{10} & $\begin{array}{l}\text { Median } \\
\text { Min. }\end{array}$ & $\begin{array}{l}4.5 \\
29.0\end{array}$ \\
\hline & J16344 & & & Max. & 72.0 \\
\hline & & $\begin{array}{l}\text { Lognormal distribution? } \\
\text { r-squared is: } \\
\text { Recommencations: } \\
\text { Use lognormal distribution. }\end{array}$ & 0.923 & $\begin{array}{l}\text { Normal distribution? } \\
\text { r-squared is: }\end{array}$ & 0.901 \\
\hline & & UCL (Land's method) is & \multicolumn{2}{|l|}{58.2} & \\
\hline $\begin{array}{l}\text { DATA } \\
6.1\end{array}$ & 1D & \multicolumn{3}{|c|}{ Chromium $95 \%$ UCL Calculation } & \\
\hline & J16332 & & & & \\
\hline 5.0 & $J 16335$ & Number of samples & \multirow{3}{*}{10} & Uncẹnsored values & \\
\hline $\begin{array}{l}7.2 \\
7.3\end{array}$ & $\begin{array}{l}\mathrm{J} 16336 \\
\mathrm{J1633}\end{array}$ & $\begin{array}{l}\text { Uncensored } \\
\text { Censored }\end{array}$ & & $\begin{array}{r}\text { Mean } \\
\text { Lonnormal mean }\end{array}$ & $\begin{array}{l}6.7 \\
6.7\end{array}$ \\
\hline 7.6 & J16338 & Detection limit or PQL. & & Std. devn. & 0.95 \\
\hline 7.3 & J16339 & Metrod detection limit & & Median & 6.9 \\
\hline 8.1 & J16340 & TOTAL. & \multirow{2}{*}{10} & Min. & 5.0 \\
\hline \multirow{5}{*}{6.6} & J16341 & & & Max. & 8.1 \\
\hline & & & \multirow{3}{*}{0.95} & & \\
\hline & & $\begin{array}{l}\text { Lognormal distribution? } \\
\text { r-squared is: }\end{array}$ & &  & 0.97 \\
\hline & & $\begin{array}{l}\text { Recommendataions: } \\
\text { Use lognoormal distribution. }\end{array}$ & & & \\
\hline & & UCL (Land's me & & & \\
\hline
\end{tabular}


Washington Closure Hanfor

Originator H.M. Sulloway AWW

Project $\frac{100 \text { - F Field Remediation }}{\text { Subject }}$

\begin{tabular}{|c|c|c|c|c|c|}
\hline \multicolumn{2}{|c|}{ Southeast Excavation } & \multicolumn{4}{|c|}{ Ecology Soft } \\
\hline DATA & 10 & Copper 95\%l & $\overline{L C a}$ & ulation & \\
\hline & J16332 & & & & \\
\hline & J16335 & Number of samples & & Uncensored values & \\
\hline 13.3 & J16336 & Uncensored & 10 & Mean & 11.9 \\
\hline 13.3 & J16337 & Censored & & Lognomal mean & 11.9 \\
\hline 11.4 & J16338 & Defection limit or $P Q L$ & & Std. devn. & 1.17 \\
\hline 11.1 & J16339 & Method detection limit & & Median & 12.0 \\
\hline 13.1 & $\begin{array}{lll}J 16340 \\
116341\end{array}$ & TOTAL & 10 & Min. & 10.0 \\
\hline $\begin{array}{l}10.6 \\
10.0\end{array}$ & J16342 & & & & \\
\hline
\end{tabular}

L.ognormal distribution?
r-squared is: $\quad 0.95 \quad \begin{aligned} & \text { Normal distribution? } \\ & r \text {-squared is: }\end{aligned} 0.96$ Recommendation

UCL. (Land's method) is 12

\begin{tabular}{|c|c|c|c|c|c|}
\hline DATA & ID & Nickel 95\%U & LCal & lation & \\
\hline & & & & & \\
\hline 7.0 & $J 16335$ & Number of samples & & Uncensored values & \\
\hline & $\begin{array}{l}J 16336 \\
114637\end{array}$ & Uncensored & 10 & Mean & \\
\hline $\begin{array}{l}8.8 \\
9.9\end{array}$ & $\begin{array}{l}J 16337 \\
J 16338\end{array}$ & $\begin{array}{l}\text { Censored } \\
\text { Detection limit or } \mathrm{PQL}\end{array}$ & & $\begin{array}{l}\text { Lognormal mean } \\
\text { Std deyn. }\end{array}$ & $\begin{array}{l}8.7 \\
1.0\end{array}$ \\
\hline & $\begin{array}{ll}J 16339 \\
\end{array}$ & Method detection linit & & Median & \\
\hline 9.1 & $\mathrm{~J} 16340$ & TOTAL & 10 & Min. & \\
\hline 7.7 & J16341 & & & Max. & 11 \\
\hline
\end{tabular}

Lognormal distribution?

Recommendations:

Use lognormal distribution

UCL (Land's method) is 9.3
CALCULATION SHEET

\author{
Date $\frac{04 / 03 / 08}{14655}$
}

Calc. No. O100F-CA-V0350
Checked

Rev. No. $\frac{413 / 08}{\text { Dote }}+\frac{130}{12 \text { of } 16}$
Sheet No.

\begin{tabular}{|c|c|c|c|c|c|}
\hline DATA & & Lead $95 \%$ UCL Ca & Iculatio & & \\
\hline 2.9 & J16332 & & & & \\
\hline & $\begin{array}{lll}J 16335 \\
16336\end{array}$ & Number of sarnples & & Uncensored values & \\
\hline $\begin{array}{l}3.1 \\
2.6\end{array}$ & 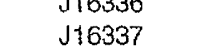 & $\begin{array}{l}\text { Uncensored } \\
\text { Censored }\end{array}$ & 10 & $\begin{array}{c}\text { Mean } \\
\text { Lognormal mean }\end{array}$ & $\begin{array}{l}4.6 \\
4.5\end{array}$ \\
\hline 2.8 & J16338 & Detection limit or $P Q L$ & & Stcd devn. & 2.9 \\
\hline 5.5 & J16339 & Method detection limit & & Median & 3.1 \\
\hline $\begin{array}{l}2.6 \\
9.6\end{array}$ & $\begin{array}{l}J 16340 \\
\text { J16341 }\end{array}$ & TOTAL & 10 & $\begin{array}{l}\text { Min. } \\
\text { Max. }\end{array}$ & $\begin{array}{r}2.6 \\
10\end{array}$ \\
\hline 3.3 & J16342 & & & & \\
\hline & & $\begin{array}{l}\text { Lognormal distribution? } \\
\text { rssuared is: } \\
\text { Recommendations: }\end{array}$ & 0.79 & $\begin{array}{l}\text { Normal distribution? } \\
r \text {-squared is: }\end{array}$ & 0.71 \\
\hline & jecl BOTH iognor & And normal distributions. & & & \\
\hline & & $C L$ (based on Z-statistic) is & 6.1 & & \\
\hline $\begin{array}{l}\text { DATA } \\
29.8\end{array}$ & $\begin{array}{c}10 \\
J 16333 / 16334\end{array}$ & Vanadium $95 \%$ U & CLCalc & uration & \\
\hline 28.0 & $\mathrm{J16332}$ & & & & \\
\hline 25.5 & J16335 & Number of samples & & Uncensored values & \\
\hline 38.9 & J16336 & Uncensorec & 10 & Mean & 33.7 \\
\hline $\begin{array}{l}33.3 \\
257\end{array}$ & $\begin{array}{lll}J 16337 \\
J 1638\end{array}$ & $\begin{array}{c}\text { Censorec } \\
\text { Detection limit or POI }\end{array}$ & & Lognormal mean & $\begin{array}{r}33.8 \\
740\end{array}-12$ \\
\hline $\begin{array}{l}25.7 \\
38.3\end{array}$ & $\begin{array}{l}J 163338 \\
J 1639\end{array}$ & $\begin{array}{l}\text { Dethod ditetection limit } \\
\text { Methen }\end{array}$ & & $\begin{array}{l}\text { sto. cevn. } \\
\text { Median. }\end{array}$ & $\begin{array}{l}.42 \\
32.4\end{array}$ \\
\hline 31.4 & J16340 & TOTAL & 10 & & \\
\hline 36.4 & J16341 & & & Max. & 49.7 \\
\hline 49.7 & $\mathrm{~J} 16342$ & & & & \\
\hline & & $\begin{array}{l}\text { Lognormal distribution? } \\
\text { r-squared is: } \\
\text { Pecommendations }\end{array}$ & 0.96 & $\begin{array}{l}\text { Nornal distribution? } \\
\text { r-squared is: }\end{array}$ & 0.92 \\
\hline & & Use & & & \\
\hline & & UCL (Land's method) is & 38.5 & & \\
\hline
\end{tabular}




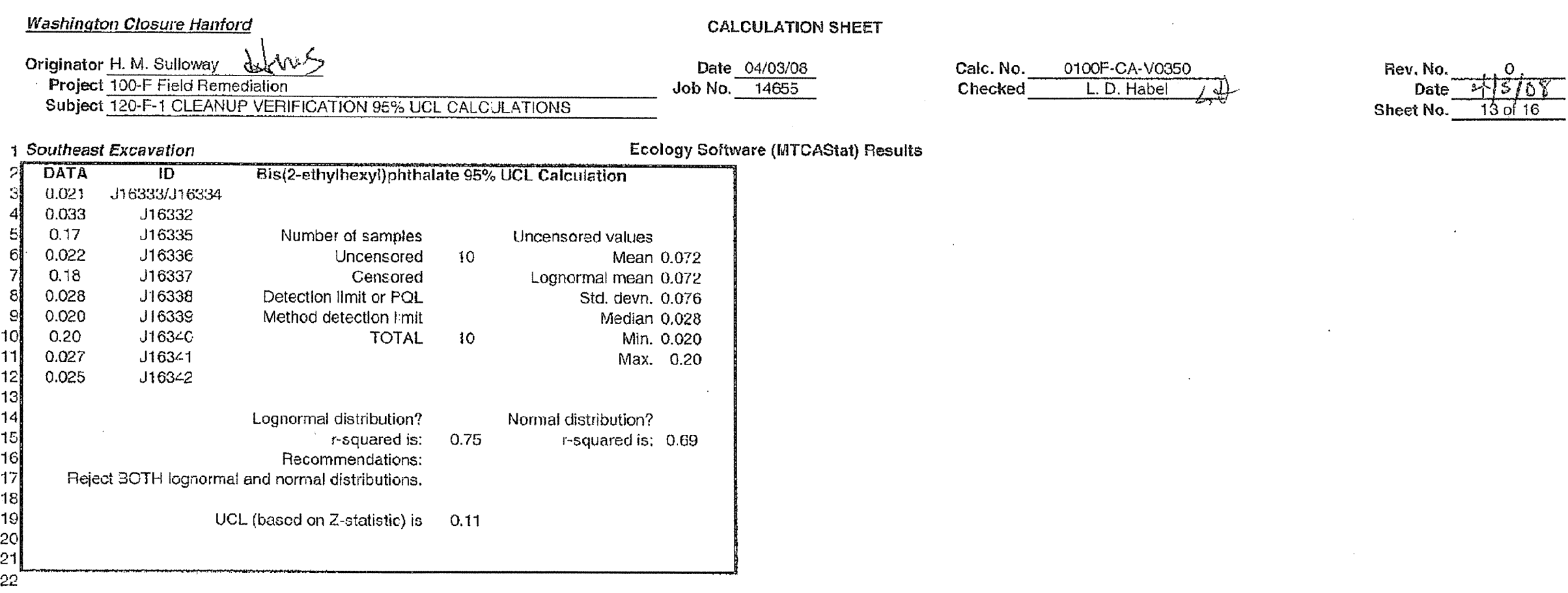




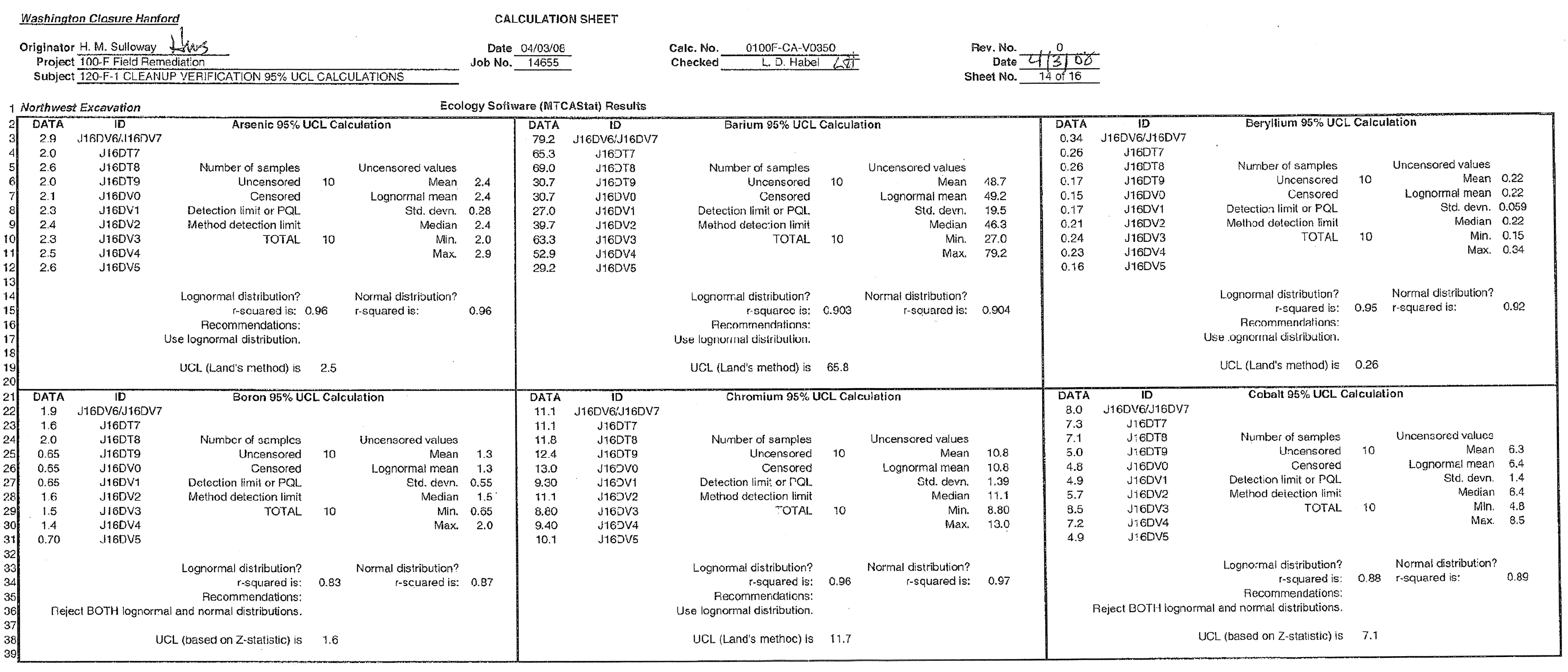




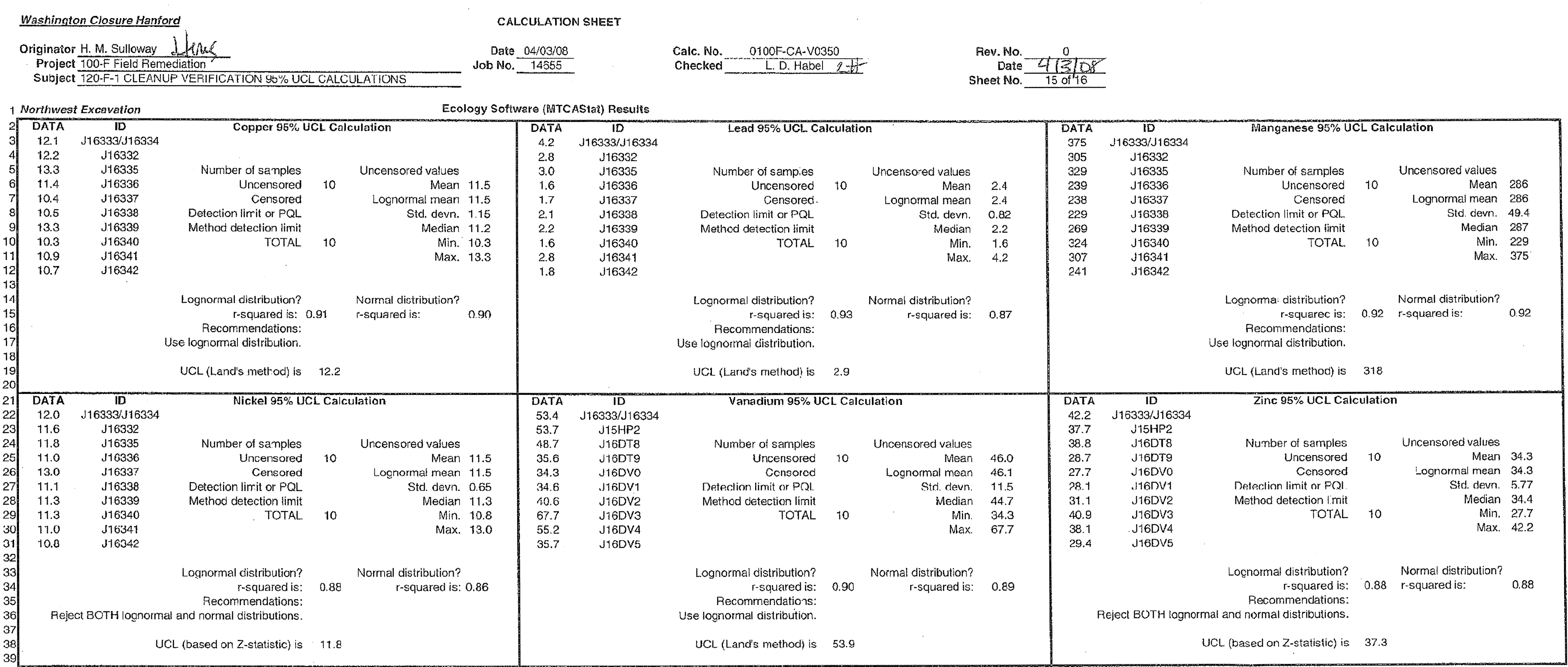


Washington Closure Hanford

Originaior H.M. Sulloway ollpey

Project $\frac{100-\mathrm{F} \text { Field Remediation }}{120-\mathrm{F}-1 \text { CLEANUP VERIFICA }}$

TANUP VERIFICATION 95\% UCL CALCULATIONS

Northwest Excavation

\begin{tabular}{|c|c|c|c|c|c|}
\hline \multirow{2}{*}{\multicolumn{2}{|c|}{$\begin{array}{l}\text { Northwest Excavation } \\
\text { DATA }\end{array}$}} & \multirow{2}{*}{\multicolumn{2}{|c|}{ Sulfate $95 \%$ UCL Calculation }} & & \\
\hline & & & & & \\
\hline 4.9 & J16333/J16334 & & & & \\
\hline $\begin{array}{l}3.9 \\
3.9\end{array}$ & J16332 & Number of samples & & Uncensored values & \\
\hline $\begin{array}{l}0.9 \\
1.1\end{array}$ & J16336 & Uncensored & 10 & Mean & 4.7 \\
\hline 4.0 & Ј16337 & Censored & & Loghormal mean & 4.8 \\
\hline 5.4 & Ј16338 & Detection limit or PQL. & & Std. deun. & 3.4 \\
\hline 3.3 & Ј16339 & Method detection limit & & Median & 4.0 \\
\hline 1.3 & J16340 & TOTAL & 10 & Min. & t.1 \\
\hline 13 & Ј 6341 & & & Max. & 13 \\
\hline 5.3 & J16342 & & & & \\
\hline & & $\begin{array}{l}\text { Lognormal distribution? } \\
\text { r-squared is: } \\
\text { Recommendations: }\end{array}$ & 0.88 & $\begin{array}{l}\text { Normal distribution? } \\
\text { r-squared is: }\end{array}$ & 0.74 \\
\hline & & & & & \\
\hline & & ICL (based on Z-statistic) is & 6.4 & & \\
\hline
\end{tabular}

Calc. No. O100F-CA-V0350

Calculations

Date $\frac{04 / 03 / 08}{14655}$

D. Habel LIf

Rev. No. $\frac{0}{4 / 3108}$

\section{Bis(2-ethylhexyl)phthalate $95 \%$ UCL-Calculation}

\begin{tabular}{|c|c|c|c|c|c|}
\hline \multirow{3}{*}{$\begin{array}{l}\overline{D A T A} \\
0.023 \\
0.025 \\
0.10\end{array}$} & & Bis(2-ethyihexyl)phthalate 95 & $\mathrm{WUCL}$ & Calculation & \\
\hline & J16333/J16334 & & & & \\
\hline & J16335 & Number of samples & & Uncensored values & \\
\hline 0.084 & J16336 & Uncensored & 10 & Mean & 0.060 \\
\hline 0.072 & Ј16337 & Censored & & Lognormal mean & 0.060 \\
\hline 0.031 & J16336 & Detection limit or PQL. & & Std. devn. & 0.047 \\
\hline 0.023 & $J 16339$ & Method detection limit & & Median & 0.048 \\
\hline 0.17 & J16340 & TOTAL & 10 & Min. & 0.023 \\
\hline 0,064 & J16341 & & & Max. & 0.17 \\
\hline \multirow[t]{4}{*}{0.029} & J16342 & & & & \\
\hline & & $\begin{array}{l}\text { Lognomal distribution? } \\
\text { r-squared is: }\end{array}$ & 0.91 & $\begin{array}{l}\text { Normal distribution? } \\
r \text { squared is: }\end{array}$ & 0.82 \\
\hline & & Recommendations: & & & \\
\hline & & UCL (Land's method) is & 0.12 & & \\
\hline
\end{tabular}


Attachment 1. 120-F-1 Verification Sampling Results.

\begin{tabular}{|c|c|c|c|c|c|c|c|c|c|c|c|c|c|c|c|c|c|c|c|c|c|c|c|}
\hline \multirow{2}{*}{$\begin{array}{c}\text { Saniple } \\
\text { Location }\end{array}$} & \multirow{2}{*}{$\begin{array}{c}\text { HEIS } \\
\text { Number }\end{array}$} & \multirow{2}{*}{$\begin{array}{c}\text { Sample } \\
\text { Date }\end{array}$} & \multicolumn{3}{|c|}{ Aluminum } & \multicolumn{3}{|c|}{ Antimony } & \multicolumn{3}{|c|}{ Arsenic } & \multicolumn{3}{|c|}{ Barium } & \multicolumn{3}{|c|}{ Beryllium } & \multicolumn{3}{|c|}{ Boron } & \multicolumn{3}{|c|}{ Cadmium } \\
\hline & & & $\mathrm{mg} / \mathrm{kg}$ & $Q$ & $\mathrm{PQL}$ & $\mathrm{mg} / \mathrm{kg}$ & $Q$ & PQL & $\mathrm{mg} / \mathrm{kg}$ & Q & $\mathrm{PQL}$ & $\mathrm{mg} / \mathrm{kg}$ & $Q$ & $\mathrm{PQL}$ & $\mathrm{mg} / \mathrm{kg}$ & $Q$ & $\mathrm{PQL}$ & $\mathrm{mg} / \mathrm{kg}$ & Q & PQL & $\mathrm{mg} / \mathrm{kg}$ & $Q$ & PQL \\
\hline SE-1 & $\mathrm{J} 16332$ & $12 / 17 / 2007$ & 4310 & C & 11.4 & 0.85 & $\mathrm{U}$ & 0.85 & 3.3 & & 1.4 & 28.8 & $\vec{C}$ & 0.28 & 0.50 & & 0.14 & 5.6 & & 1.4 & 0.14 & $\mathrm{U}$ & 0.14 \\
\hline SE-2 & 316333 & $12 / 17 / 2007$ & 4960 & $C$ & 10.5 & 0.79 & $\mathrm{U}$ & 0.79 & 1.8 & & 1.3 & 49.9 & $C$ & 0.26 & 0.56 & & 0.13 & 1.7 & & 1.3 & 0.13 & $\mathrm{U}$ & 0.13 \\
\hline Duplicate of & & & & & & & & & & & & & & & & & & & & & & & \\
\hline $\mathrm{J} 16333$ & $\mathrm{~J} 16334$ & $12 / 17 / 2007$ & 4550 & C & 11.9 & 0.9 & $\mathrm{U}$ & 0.9 & 1.5 & & 1.5 & 44.1 & C & 0.30 & 0.43 & & 0.15 & 1.8 & & 1.5 & 0.15 & $\mathrm{U}$ & 0.15 \\
\hline SE-3 & $\mathrm{J} 16335$ & $12 / 17 / 2007$ & 4290 & $\mathrm{C}$ & 11.6 & 0.87 & $\mathrm{U}$ & 0.87 & 2.2 & & 1.4 & 30.4 & $C$ & 0.29 & 0.41 & & 0.14 & 1.4 & U & 1.4 & 0.14 & $\mathrm{U}$ & 0.14 \\
\hline SE-4 & $J 16336$ & $12 / 17 / 2007$ & 6690 & $\mathrm{C}$ & 11.5 & 0.86 & $\mathrm{U}$ & 0.86 & 2.5 & & 1.4 & 54.7 & C & 0.29 & 0.69 & & 0.14 & 1.4 & & 1.4 & 0.14 & $\mathrm{U}$ & 0.14 \\
\hline SE-5 & $\mathrm{J} 16337$ & $12 / 17 / 2007$ & 5070 & & 11.4 & 0.85 & $\mathrm{U}$ & 0.85 & 3.2 & & 1.4 & 36.0 & & 0.28 & 0.58 & & 0.14 & 5.6 & & 1.4 & 0.14 & $\mathrm{U}$ & 0.14 \\
\hline SE-6 & .116338 & $12 / 17 / 2007$ & 5100 & & 11.0 & 0.82 & $\mathrm{U}$ & 0.82 & 2.3 & & 1.4 & 36.3 & & 0.27 & 0.47 & & 0.14 & 1.4 & $\mathrm{U}$ & 1.4 & 0.14 & $\mathrm{U}$ & 0.14 \\
\hline SE-7 & $\mathrm{J16339}$ & $12 / 17 / 2007$ & 5430 & & 12.0 & 0.9 & $\mathrm{U}$ & 0.9 & 2.4 & & 1.5 & 52.3 & & 0.30 & 0.70 & & 0.15 & 1.8 & & 1.5 & 0.15 & $\mathrm{U}$ & 0.15 \\
\hline SE-8 & $J 16340$ & $12 / 17 / 2007$ & 4910 & & 10.6 & 0.8 & $\mathrm{U}$ & 0.8 & 2.4 & & 1.3 & 29.3 & & 0.27 & 0.54 & & 0.13 & 1.3 & U & 1.3 & 0.13 & $\mathrm{U}$ & 0.13 \\
\hline SE-9 & $\mathrm{J1} 16341$ & $12 / 17 / 2007$ & 5130 & & 12.4 & 0.93 & $\mathrm{U}$ & 0.93 & 2.5 & & 1.5 & 70.2 & & 0.31 & 0.77 & & 0.15 & 2.4 & & 1.5 & 0.15 & $\mathrm{U}$ & 0.15 \\
\hline SE-10 & $\mathrm{J16342}$ & $12 / 17 / 2007$ & 6320 & & 12.7 & 0.95 & $\mathrm{U}$ & 0.95 & 2.8 & & 1.6 & 72.0 & & 0.32 & 0.99 & & 0.16 & 1.6 & U & 1.6 & 0.16 & $\mathrm{U}$ & 0.16 \\
\hline$N W-1$ & J16DT7 & $3 / 18 / 2008$ & 6530 & & 10.9 & 0.82 & $\mathrm{U}$ & 0.82 & 2.0 & & 1.4 & 65.3 & & 0.27 & 0.26 & & 0.14 & 1.6 & & 1.4 & 0.14 & $\mathrm{U}$ & 0.14 \\
\hline $\mathrm{NW}-2$ & J16DT8 & $3 / 18 / 2008$ & 7360 & & 11.1 & 0.83 & $\mathrm{U}$ & 0.83 & 2.6 & & 1.4 & 69.0 & & 0.28 & 0.26 & & 0.14 & 2.0 & & 1.4 & 0.14 & $\mathrm{U}$ & 0.14 \\
\hline NW-3 & J16DT9 & $3 / 18 / 2008$ & 5120 & & 10.4 & 0.78 & $\mathrm{U}$ & 0.78 & 2.0 & & 1.3 & 30.7 & & 0.26 & 0.17 & & 0.13 & 1.3 & U & 1.3 & 0.13 & $\mathrm{U}$ & 0.13 \\
\hline$N W-4$ & .116DV0 & $3 / 18 / 2008$ & 5050 & & 10.4 & 0.78 & $\mathrm{U}$ & 0.78 & 2.1 & & 1.3 & 30.7 & & 0.26 & 0.15 & & 0.13 & 1.3 & U & 1.3 & 0.13 & $\mathrm{U}$ & 0.13 \\
\hline $\mathrm{NW}-5$ & J16DVI & $3 / 18 / 2008$ & 4860 & & 10.2 & 0.91 & & 0.76 & 2.3 & & 1.3 & 27.0 & & 0.26 & 0.17 & & 0.13 & 1.3 & U & 1.3 & 0.13 & $\mathrm{U}$ & 0.13 \\
\hline NW-6 & $.116 \mathrm{DV} 2$ & $3 / 18 / 2008$ & 5990 & & 10.1 & 0.76 & $\mathrm{U}$ & 0.76 & 2.4 & & 1.3 & 39.7 & & 0.25 & 0.21 & & 0.13 & 1.6 & & 1.3 & 0.13 & $\mathrm{U}$ & 0.13 \\
\hline $\mathrm{NW}-7$ & J16DV3 & $3 / 18 / 2008$ & 4660 & & 10.6 & 0.79 & $\mathrm{U}$ & 0.79 & 2.3 & & 1.3 & 63.3 & & 0.26 & 0.24 & & 0.13 & 1.5 & & 1.3 & 0.13 & $\mathrm{U}$ & 0.13 \\
\hline$N W-8$ & $\mathrm{J16DV4}$ & $3 / 18 / 2008$ & 5400 & & 11.0 & 0.83 & $\mathrm{U}$ & 0.83 & 2.5 & & 1.4 & 52.9 & & 0.28 & 0.23 & & 0.14 & 1.4 & & 1.4 & 0.14 & $\mathrm{U}$ & 0.14 \\
\hline NW-9 & J16DV5 & $3 / 18 / 2008$ & 5030 & & 11.4 & 0.85 & $\mathrm{U}$ & 0.85 & 2.6 & & 1.4 & 29.2 & & 0.28 & 0.16 & & 0.14 & 1.4 & U & 1.4 & 0.14 & $\mathrm{U}$ & 0.14 \\
\hline Duplicate of & & & & & & & & & & & & & & & & & & & & & & & \\
\hline $116 \mathrm{DV} 6$ & J16DV7 & $3 / 18 / 2008$ & 7270 & & 11.5 & 0.86 & U & 0.86 & 3.0 & & 1.4 & 81.0 & & 0.29 & 0.34 & & 0.14 & 1.9 & & 1.4 & 0.14 & $u$ & 0.14 \\
\hline $\mathrm{NW}-10$ & $316 \mathrm{DV} 6$ & $3 / 18 / 2008$ & 7520 & & 11.6 & 0.87 & $\mathrm{U}$ & 0.87 & 2.7 & & 1.4 & 77.4 & & 0.29 & 0.34 & & 0.14 & 1.9 & & 1.4 & 0.14 & $\mathrm{U}$ & 0.14 \\
\hline Equip blank & .116354 & $12 / 17 / 2007$ & 80.7 & & 3.7 & 0.28 & $\mathrm{U}$ & 0.28 & 0.46 & $\mathrm{U}$ & 0.46 & 1.7 & & 0.09 & 0.05 & $\mathrm{U}$ & 0.05 & 0.46 & $\mathrm{U}$ & 0.46 & 0.05 & $\mathrm{U}$ & 0.05 \\
\hline Equip blank & J19DT6 & $3 / 18 / 2008$ & 49.7 & & 3 & 0.24 & $\mathrm{U}$ & 0.24 & 0.4 & $\mathrm{U}$ & 0.4 & 1 & & 0.08 & 0.04 & $\mathrm{U}$ & 0.04 & 0.4 & $\mathrm{U}$ & 0.4 & 0.04 & $\mathrm{U}$ & 0.04 \\
\hline
\end{tabular}

Attachinent

Originator H.M. Sullowas (c) the Date $\frac{1 \text { of } 18}{04 / 03 / 08}$

Checked L. D. Habel If Date $04 / 03 / 08$

Calc. No. 0100F-CA-V0350 Rev. No. 
Attachment 1. 120-F-1 Verification Sampling Results.

\begin{tabular}{|c|c|c|c|c|c|c|c|c|c|c|c|c|c|c|c|c|c|c|c|c|c|c|c|}
\hline \multirow{2}{*}{$\begin{array}{c}\text { Sample } \\
\text { Location }\end{array}$} & \multirow{2}{*}{$\begin{array}{c}\text { Hers } \\
\text { Number }\end{array}$} & \multirow{2}{*}{$\begin{array}{c}\text { Sample } \\
\text { Date }\end{array}$} & \multicolumn{3}{|c|}{ Calcium } & \multicolumn{3}{|c|}{ Chromium } & \multicolumn{3}{|c|}{ Cobalt } & \multicolumn{3}{|c|}{ Copper } & \multicolumn{3}{|c|}{ Iron } & \multicolumn{3}{|c|}{ Lead } & \multicolumn{3}{|c|}{ Magnesium } \\
\hline & & & $\mathrm{mg} / \mathrm{kg}$ & $Q$ & $\mathrm{PQL}$ & $\mathrm{mg} / \mathrm{kg}$ & $Q$ & $\mathrm{PQL}$ & $\mathrm{mg} / \mathrm{kg}$ & $\mathrm{Q}$ & $\mathrm{PQL}$ & $\mathrm{mg} / \mathrm{kg}$ & $Q$ & $\mathrm{PQL}$ & $\mathrm{mg} / \mathrm{kg}$ & $Q$ & $\mathrm{PQL}$ & $\mathrm{mg} / \mathrm{kg}$ & Q & $\mathrm{PQL}$ & $\mathrm{mg} / \mathrm{kg}$ & Q & PQL \\
\hline SE-1 & $\mathrm{J} 16332$ & $12 / 17 / 2007$ & 2630 & C & 11.4 & 6.4 & & 0.57 & 4.3 & & 0.57 & 12.3 & C & 0.57 & 10700 & $\begin{array}{ll}\mathrm{C} \\
\end{array}$ & 12.8 & 2.7 & & 0.85 & 3220 & & 7.1 \\
\hline SE-2 & $\mathrm{J} 16333$ & $12 / 17 / 2007$ & 4920 & C & 10.5 & 6 & & 0.53 & 4.7 & & 0.53 & 12.3 & C & 0.53 & 13800 & \begin{tabular}{|l|}
$\mathrm{C}$ \\
\end{tabular} & 11.9 & 11.1 & & 0.79 & 3610 & & 6.6 \\
\hline \multicolumn{24}{|c|}{\begin{tabular}{l|l} 
Duplicate of & \\
\end{tabular}} \\
\hline II6333 & $\mathrm{J} 16334$ & $12 / 17 / 2007$ & 5050 & C & 11.9 & 6.1 & & 0.6 & 4.0 & & 0.60 & 11.6 & c & 0.6 & 10700 & $c$ & 13.4 & 9.0 & & 0.90 & 3240 & & 7.5 \\
\hline $\mathrm{SE}-3$ & $\mathrm{~J} 16335$ & $12 / 17 / 2007$ & 2590 & $\mathrm{C}$ & 11.6 & 5 & & 0.58 & 3.8 & & 0.58 & 12.1 & C & 0.58 & 10300 & \begin{tabular}{|l|}
$\mathrm{C}$ \\
\end{tabular} & 13 & 2.8 & & 0.87 & 2990 & & 7.2 \\
\hline SE-4 & $\pi 16336$ & $12 / 17 / 2007$ & 5550 & C & 11.5 & 7.2 & & 0.57 & 6.1 & & 0.57 & 13.3 & C & 0.57 & 16400 & \begin{tabular}{|l|}
$\mathrm{C}$ \\
\end{tabular} & 12.9 & 3.7 & & 0.86 & 3800 & & 7.2 \\
\hline SE-5 & .116337 & $12 / 17 / 2007$ & 3440 & C & 11.4 & 7.3 & & 0.57 & 4.6 & & 0.57 & 13.3 & & 0.57 & 12900 & & 12.8 & 2.6 & & 0.85 & 4020 & & 7.1 \\
\hline SE-6 & $\mathrm{J} 16338$ & $12 / 17 / 2007$ & 7250 & $\mathrm{C}$ & 11 & 7.6 & & 0.55 & 4.3 & & 0.55 & 11.4 & & 0.55 & 11000 & & 12.4 & 2.8 & & 0.82 & 3610 & & 6.9 \\
\hline SE-7 & $\mathrm{J} 16339$ & $12 / 17 / 2007$ & 3880 & $\mathrm{C}$ & 12 & 7.3 & & 0.6 & 4.9 & & 0.6 & 11.1 & & 0.6 & 15000 & & 13.5 & 5.5 & & 0.90 & 3710 & & 7.5 \\
\hline SE-8 & 116340 & $12 / 17 / 2007$ & 3100 & $\mathrm{C}$ & 10.6 & 8.1 & & 0.53 & 4.2 & & 0.53 & 13.1 & & 0.53 & 12300 & & 12 & 2.6 & & 0.80 & 3580 & & 6.6 \\
\hline SE-9 & $\mathrm{J} 16341$ & $12 / 17 / 2007$ & 4050 & C & 12.4 & 5.7 & & 0.62 & 5.4 & & 0.62 & 10.4 & & 0.62 & 16300 & & 13.9 & 9.6 & & 0.93 & 3430 & & 7.7 \\
\hline$S E-10$ & $\mathrm{~J} 16342$ & $12 / 17 / 2007$ & 3650 & C & 12.7 & 6.6 & & 0.64 & 6.1 & & 0.64 & 10.0 & & 0.64 & 19900 & & 14.3 & 3.3 & & 0.95 & 4100 & & 7.9 \\
\hline$N W-1$ & J16DT7 & $3 / 18 / 2008$ & 3750 & & 10.9 & 11.1 & & 0.54 & 7.3 & & 0.54 & 12.2 & & 0.54 & 21400 & & 12.2 & 2.8 & & 0.82 & 4370 & & 6.8 \\
\hline$N W-2$ & $316 \mathrm{DT} 8$ & $3 / 18 / 2008$ & 3910 & & 11.1 & 11.8 & & 0.56 & 7.1 & & 0.56 & 13.3 & & 0.56 & 21100 & & 12.5 & 3.0 & & 0.83 & 4360 & & 6.9 \\
\hline $\mathrm{NW}-3$ & $316 \mathrm{DT} 9$ & $3 / 18 / 2008$ & 4150 & & 10.4 & 12.4 & & 0.52 & 5.0 & & 0.52 & 11.4 & & 0.52 & 14800 & & 11.7 & 1.6 & & 0.78 & 3820 & & 6.5 \\
\hline NW-4 & J16DV0 & $3 / 18 / 2008$ & 4860 & & 10.4 & 13.0 & & 0.52 & 4.8 & & 0.52 & 10.4 & & 0.52 & 14100 & & 11.7 & 1.7 & & 0.78 & 4020 & & 6.5 \\
\hline $\mathrm{NW}-5$ & $116 \mathrm{DVI}$ & \begin{tabular}{|l|}
$3 / 18 / 2008$ \\
\end{tabular} & 4030 & & 10.2 & 9.3 & & 0.51 & 4.9 & & 0.51 & 10.5 & & 0.51 & 14100 & & 11.5 & 2.1 & & 0.76 & 3600 & & 6.4 \\
\hline$N W-6$ & J16DV2 & $3 / 18 / 2008$ & 3850 & & 10.1 & 11.1 & & $0.5 !$ & 5.7 & & 0.51 & 13.3 & & 0.51 & 16900 & & 11.4 & 2.2 & & 0.76 & 4000 & & 6.3 \\
\hline$N W-7$ & J16DV3 & $3 / 18 / 2008$ & 5060 & & 10.6 & 8.8 & & 0.53 & 8.5 & & 0.53 & 10.3 & & 0.53 & 24600 & & 11.9 & 1.6 & & 0.79 & 4330 & & 6.6 \\
\hline $\mathrm{NW}-\mathrm{S}$ & $\mathrm{J16DV4}$ & $3 / 18 / 2008$ & 4020 & & 11.0 & 9.4 & & 0.55 & 7.2 & & 0.55 & 10.9 & & 0.55 & 21300 & & 12.4 & 2.8 & & 0.83 & 4140 & & 6.9 \\
\hline NW-9 & J16DV5 & $3 / 18 / 2008$ & 4460 & & 11.4 & 10.1 & & 0.57 & 4.9 & & 0.57 & 10.7 & & 0.57 & 14900 & & 12.8 & 1.8 & & 0.85 & 3700 & & 7.1 \\
\hline \multicolumn{24}{|l|}{ Duplicate of } \\
\hline J16DV6 & J16DV7 & $3 / 18 / 2008$ & 3930 & & 11.5 & 10.6 & & 0.58 & 7.8 & & 0.58 & 12.6 & & 0.58 & 22600 & & 12.9 & 4.4 & & 0.86 & 4180 & & 7.2 \\
\hline NW-10 & J16DV6 & \begin{tabular}{|l|l|l|}
$3 / 2008$ \\
\end{tabular} & 3820 & & 11.6 & 11.6 & & 0.58 & 8.2 & & 0.58 & 11.6 & & 0.58 & 23500 & & 13.0 & 3.9 & & 0.87 & 4220 & & 7.2 \\
\hline Equip blank & $\mathrm{J16354}$ & \begin{tabular}{|l|}
$12 / 17 / 2007$ \\
\end{tabular} & 23.9 & $\mathrm{C}$ & 3.7 & 0.19 & $\mathrm{U}$ & 0.19 & 0.19 & $\mathrm{U}$ & 0.19 & 0.25 & & 0.19 & 156 & & 4.2 & 0.34 & & 0.28 & 10.3 & & 2.3 \\
\hline Equip blank & J19DT6 & $3 / 18 / 2008$ & 20.6 & & 3.2 & 0.16 & $\mathrm{U}$ & 0.16 & 0.16 & $U$ & 0.16 & 0.16 & U & 0.16 & 124 & & 3.6 & 0.24 & $\mathrm{U}$ & 0.24 & 10.1 & & 2 \\
\hline
\end{tabular}

Attechnent 1 Sheet No. $\frac{2 \text { of } 18}{04 / 03 / 08}$ Originator M. Sulloway Crigin

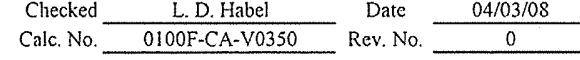


Attachment 1. 120-F-1 Verification Sampling Results.

\begin{tabular}{|c|c|c|c|c|c|c|c|c|c|c|c|c|c|c|c|c|c|c|c|c|c|c|c|}
\hline \multirow{2}{*}{$\begin{array}{c}\text { Sample } \\
\text { Location }\end{array}$} & \multirow{2}{*}{$\begin{array}{c}\text { HEIS } \\
\text { Number }\end{array}$} & \multirow{2}{*}{$\begin{array}{c}\text { Sample } \\
\text { Date }\end{array}$} & \multicolumn{3}{|c|}{ Manganese } & \multicolumn{3}{|c|}{ Mercury } & \multicolumn{3}{|c|}{ Molybdenum } & \multicolumn{3}{|c|}{ Nickel } & \multicolumn{3}{|c|}{ Potassium } & \multicolumn{3}{|c|}{ Selenium } & \multicolumn{3}{|c|}{ Silicon } \\
\hline & & & $\mathrm{mg} / \mathrm{kg}$ & $Q$ & POL & $\mathrm{mg} / \mathrm{kg}$ & Q & PQL & $\mathrm{mg} / \mathrm{kg}$ & $Q$ & $\mathrm{PQL}$ & $\mathrm{mg} / \mathrm{kg}$ & $Q$ & $\mathrm{PQL}$ & $\mathrm{mg} / \mathrm{kg}$ & $\mathrm{Q}$ & $\mathrm{PQL}$ & $\mathrm{mg} / \mathrm{kg}$ & $\mathrm{Q}$ & PQL & $\mathrm{mg} / \mathrm{kg}$ & $Q$ & $\mathrm{PQL}$ \\
\hline SE-1 & $\mathrm{J} 16332$ & $12 / 17 / 2007$ & 191 & & 0.11 & 0.01 & $\mathrm{U}$ & 0.01 & 0.85 & $\mathrm{U}$ & 0.85 & 8.4 & & 0.57 & 631 & & 11.4 & 1.7 & U & 1.7 & 2090 & & 11.4 \\
\hline $\mathrm{SE}-2$ & 116333 & $12 / 17 / 2007$ & 232 & & 0.11 & 0.009 & $\mathrm{U}$ & 0.009 & 0.79 & $\mathrm{U}$ & 0.79 & 8.4 & & 0.53 & 757 & & 10.5 & 1.6 & $\mathrm{U}$ & 1.6 & 1940 & & 10.5 \\
\hline Duplicate of & & & & & & & & & & & & & & & & & & & & & & & \\
\hline 16333 & 116334 & $12 / 17 / 2007$ & 198 & & 0.12 & 0.009 & $u$ & 0.009 & 0.90 & $U$ & 0.90 & 7.7 & & 0.6 & 675 & & 11.9 & 1.8 & $\mathrm{u}$ & 1.8 & 2100 & & 11.9 \\
\hline SE-3 & $\mathrm{J} 16335$ & $12 / 17 / 2007$ & 191 & & 0.12 & 0.009 & U & 0.009 & 0.87 & $\mathrm{U}$ & 0.87 & 7.0 & & 0.58 & 539 & & 11.6 & 1.7 & $\mathrm{u}$ & 1.7 & 1720 & & 11.6 \\
\hline $\mathrm{SE}-4$ & $\mathrm{J16336}$ & $12 / 17 / 2007$ & 261 & & 0.11 & 0.65 & & 0.008 & 0.86 & $\mathrm{U}$ & 0.86 & 10.5 & & 0.57 & 1040 & & 11.5 & 1.7 & $\mathrm{U}$ & 1.7 & 1650 & & 11.5 \\
\hline $\mathrm{SE}-5$ & $\mathrm{~J} 16337$ & $12 / 17 / 2007$ & 229 & & 0.11 & 0.009 & $\mathrm{U}$ & 0.009 & 0.85 & & 0.85 & 8.8 & & 0.57 & 678 & & 11.4 & 1.7 & $\mathrm{u}$ & 1.7 & 2320 & & 11.4 \\
\hline SE-6 & $\mathrm{J16338}$ & $12 / 17 / 2007$ & 206 & & 0.11 & 0.01 & & 0.01 & 0.82 & $\mathrm{U}$ & 0.82 & 9.9 & & 0.55 & 704 & & 11.0 & 1.6 & $\mathrm{U}$ & 1.6 & 1650 & & 11.0 \\
\hline SE-7 & 516339 & $12 / 17 / 2007$ & 247 & & 0.12 & 0.02 & & 0.01 & 0.90 & $\mathrm{U}$ & 0.90 & 8.4 & & 0.6 & 954 & & 12.0 & 1.8 & $\mathrm{u}$ & 1.8 & 2420 & & 12.0 \\
\hline SE-8 & .116340 & $12 / 17 / 2007$ & 216 & & 0.11 & 0.01 & $\mathrm{U}$ & 0.01 & 0.80 & $\mathrm{U}$ & 0.80 & 9.1 & & 0.53 & 784 & & 10.6 & 1.6 & $\mathrm{U}$ & 1.6 & 1860 & & 10.5 \\
\hline SE-9 & $\mathrm{J16341}$ & \begin{tabular}{|l|l|}
$12 / 17 / 2007$ \\
\end{tabular} & 270 & & 0.12 & 0.06 & & 0.009 & 0.93 & $\mathrm{U}$ & 0.93 & 7.7 & & 0.62 & 1160 & & 12.4 & 1.9 & U & 1.9 & 2900 & & 12.4 \\
\hline SE-10 & .116342 & $12 / 17 / 2007$ & 314 & & 0.13 & 0.01 & $\mathrm{U}$ & 0.01 & 0.95 & $\mathrm{U}$ & 0.95 & 8.9 & & 0.64 & 1210 & & 12.7 & 1.9 & U & 1.9 & 3200 & & $\frac{1.47}{12.7}$ \\
\hline NW-1 & $116 \mathrm{DT} 7$ & $3 / 18 / 2008$ & 305 & & 0.11 & 0.008 & U & 0.008 & 0.82 & U & 0.82 & 11.6 & & 0.54 & 1170 & & 10.9 & 1.6 & $\mathrm{U}$ & 1.6 & 387 & & 10.9 \\
\hline NW-2 & J16DT8 & $3 / 18 / 2008$ & 329 & & 0,11 & 0.01 & $\mathrm{U}$ & 0.01 & 0.83 & $\mathrm{U}$ & 0.83 & 11.8 & & 0.56 & 1230 & & 11.1 & 1.8 & & 1.7 & 423 & & 11.1 \\
\hline NW-3 & J160T9 & $3 / 18 / 2008$ & 239 & & 0.10 & 0.01 & $\mathrm{U}$ & 0.01 & 0.78 & $\mathrm{U}$ & 0.78 & 11.0 & & 0.52 & 654 & & 10.4 & 1.6 & $\mathrm{U}$ & 1.6 & 364 & & 10.4 \\
\hline NW-4 & J16DV0 & $3 / 18 / 2008$ & 238 & & 0.10 & 0.01 & $\mathrm{U}$ & 0.01 & 0.78 & $\mathrm{U}$ & 0.78 & 13.0 & & 0.52 & 617 & & 10.4 & 1.6 & $\mathrm{U}$ & 1.6 & 342 & & 10.4 \\
\hline NW-5 & J16DV1 & $3 / 18 / 2008$ & 229 & & 0.10 & 0.009 & $\mathrm{U}$ & 0.009 & 0.76 & $\bar{U}$ & 0.76 & 11.1 & & 0.51 & 584 & & 10.2 & 1.5 & U & 1.5 & 341 & & 10.2 \\
\hline NW-6 & $\mathrm{J16DV} 2$ & $3 / 18 / 2008$ & 269 & & 0.10 & 0.009 & U & 0.009 & 0.76 & $\mathrm{U}$ & 0.76 & 11.3 & & 0.51 & 730 & & 10.1 & 1.5 & $\mathrm{u}$ & 1.5 & 412 & & 10.1 \\
\hline$N W-7$ & II6DV3 & $3 / 18 / 2008$ & 324 & & 0.1 & 0.007 & $\mathrm{U}$ & 0.007 & 0.79 & $\mathrm{U}$ & 0.79 & 11.3 & & 0.53 & 733 & & 10.6 & 1.6 & $\mathrm{U}$ & 1.6 & 434 & & 10.6 \\
\hline NW-S & $116 \mathrm{DV} 4$ & & 307 & & 0.1 & 0.009 & $\mathrm{U}$ & 0.009 & 0.83 & $\mathrm{U}$ & 0.83 & 11 & & 0.55 & 826 & & 11.0 & 1.7 & $\mathrm{U}$ & 1.7 & 543 & & 11.0 \\
\hline NW-9 & $1160 V 5$ & $3 / 18 / 2008$ & 241 & & 0.11 & 0.008 & $\mathrm{U}$ & 0.008 & 0.85 & $\mathrm{U}$ & 0.85 & 10.8 & & 0.57 & 653 & & 11.4 & 1.7 & $\mathrm{U}$ & 1.7 & 381 & & 11.4 \\
\hline Duplica & & & & & & & & & & & & & & & & & & & & & & & \\
\hline $116 \mathrm{DV} 6$ & J16DV7 & & 371 & & 0.12 & 0.01 & $\mathrm{u}$ & 0.01 & 0.86 & $\mathrm{U}$ & 0.86 & 12.1 & & 0.58 & 1390 & & 11.5 & 1.7 & $\mathrm{u}$ & 1.7 & 495 & & 11.5 \\
\hline$N W-10$ & I16DV6 & & 378 & & 0.12 & 0.01 & $\mathrm{U}$ & 0.01 & 0.87 & $\mathrm{U}$ & 0.87 & 11.9 & & 0.58 & 1400 & & 11.6 & 1.7 & u & 1.7 & 466 & & 11.6 \\
\hline Equip blank & $J 16354$ & $12 / 17 / 2007$ & 4.2 & & 0.04 & 0.009 & $\mathrm{U}$ & 0.009 & 0.28 & $U$ & 0.28 & 0.19 & 10 & 0.19 & 37.3 & & 3.7 & 0.56 & U & 0.56 & 102 & & 3.7 \\
\hline Equip blank & J19DT6 & $3 / 18 / 2008$ & 3.2 & & 0.03 & 0.009 & $\mathrm{Uu}$ & 0.009 & 0.24 & $\mathrm{U}$ & 0.24 & 0.16 & & 0.16 & 15.2 & & 3.2 & 0.48 & U & 0.48 & 42.1 & & 3.2 \\
\hline
\end{tabular}

Attachment

Sheet No. $\quad 3$ of 18

Originator H.M. Sulloway Date

Checked L. D. Habel 
Attachment 1. 120-F-1 Verification Sampling Results.

\begin{tabular}{|c|c|c|c|c|c|c|c|c|c|c|c|c|c|c|c|c|c|c|c|c|c|c|c|}
\hline \multirow{2}{*}{$\begin{array}{c}\text { Sample } \\
\text { Location }\end{array}$} & \multirow{2}{*}{$\begin{array}{c}\text { HEIS } \\
\text { Number }\end{array}$} & \multirow{2}{*}{$\begin{array}{l}\text { Sample } \\
\text { Date }\end{array}$} & \multicolumn{3}{|c|}{ Silver } & \multicolumn{3}{|c|}{ Sodium } & \multicolumn{3}{|c|}{ Vanadium } & \multicolumn{3}{|c|}{ Zinc } & \multicolumn{3}{|c|}{$\begin{array}{c}\text { Total petroleum } \\
\text { hydrocarbons }\end{array}$} & \multicolumn{3}{|c|}{ Bromide } & \multicolumn{3}{|c|}{ Chloride } \\
\hline & & & $\mathrm{mg} / \mathrm{kg}$ & $Q$ & $\mathrm{PQL}$ & $\mathrm{mg} / \mathrm{kg}$ & $Q$ & $\mathrm{PQL}$ & $\mathrm{mg} / \mathrm{kg}$ & $\mathrm{Q}$ & $\mathrm{PQL}$ & $\mathrm{mg} / \mathrm{kg}$ & Q & $\mathrm{PQL}$ & $\mathrm{mg} / \mathrm{kg}$ & $Q$ & PQL & $\mathrm{mg} / \mathrm{kg}$ & Q & $\mathrm{PQL}$ & $\mathrm{mg} / \mathrm{kg}$ & Q & $\mathrm{PQL}$ \\
\hline SE-1 & $\mathrm{J16332}$ & $12 / 17 / 2007$ & 0.28 & $\mathrm{U}$ & 0.28 & 117 & C & 5.7 & 28 & & 0.4 & 24.8 & C & 1.7 & 137 & $\mathrm{U}$ & 137 & 2.4 & U & 2.4 & 2.4 & $\mathrm{U}$ & 2.4 \\
\hline $\mathrm{SE}-2$ & $J 16333$ & $12 / 17 / 2007$ & 0.26 & $\mathrm{U}$ & 0.26 & 200 & $C$ & 5.3 & 33.1 & & 0.37 & 30.2 & C & 1.6 & 138 & $\mathrm{U}$ & 138 & 2.4 & $\mathrm{U}$ & 2.4 & 3.1 & & 2.4 \\
\hline \multicolumn{24}{|l|}{ Duplicate of } \\
\hline 116333 & $\mathrm{~J} 16334$ & $12 / 17 / 2007$ & 0.3 & $\mathrm{U}$ & 0.3 & 200 & C & 6 & 26.5 & & 0.42 & 26 & c & 1.8 & 138 & $\mathrm{U}$ & 138 & 2.3 & $\mathrm{U}$ & 2.3 & 3.4 & & 2.3 \\
\hline SE-3 & .116335 & $12 / 17 / 2007$ & 0.29 & $\mathrm{U}$ & 0.29 & 127 & $\mathrm{C}$ & 5.8 & 25.5 & & 0.4 & 24.1 & C & 1.7 & 137 & $\mathrm{U}$ & 137 & 2.5 & U & 2.5 & 2.5 & $\mathrm{U}$ & 2.5 \\
\hline SE-4 & 316336 & \begin{tabular}{|l|}
$12 / 17 / 2007$ \\
\end{tabular} & 0.29 & $\mathrm{U}$ & 0.29 & 183 & $\mathrm{C}$ & 5.7 & 38.9 & & 0.4 & 35.9 & C & 1.7 & 143 & U & 143 & 2.7 & $\mathrm{U}$ & 2.7 & 9.7 & & 2.7 \\
\hline SE-5 & $\mathrm{J} 16337$ & \begin{tabular}{|l|}
$12 / 17 / 2007$ \\
\end{tabular} & 0.28 & $\mathrm{U}$ & 0.28 & 430 & $C$ & 5.7 & 33.3 & & 0.4 & 28.1 & C & 1.7 & 139 & $\mathrm{U}$ & 139 & 2.5 & $\mathrm{U}$ & 2.5 & 34 & & 2.5 \\
\hline SE-6 & $\mathrm{J16338}$ & $12 / 17 / 2007$ & 0.27 & $\mathrm{U}$ & 0.27 & 152 & $\mathrm{C}$ & 5.5 & 25.7 & & 0.38 & 28.4 & C & 1.6 & 140 & $\mathrm{U}$ & 140 & 2.5 & $\mathrm{U}$ & 2.5 & 2.5 & 0 & 2.5 \\
\hline SE-7 & 116339 & $12 / 17 / 2007$ & 0.3 & $\mathrm{U}$ & 0.3 & 172 & C & 6 & 38.3 & & 0.42 & 39 & C & 1.8 & 140 & $\mathrm{U}$ & 140 & 2.6 & $\mathrm{U}$ & 2.6 & 2.6 & $\mathrm{U}$ & 2.6 \\
\hline SE-8 & $\mathrm{J} 16340$ & $12 / 17 / 2007$ & 0.27 & $\mathrm{U}$ & 0.27 & 193 & C & 5.3 & 31.4 & & 0.37 & 30.9 & C & 1.6 & 139 & $\mathrm{U}$ & 139 & 2.7 & $\mathrm{U}$ & 2.7 & 2.7 & $\mathrm{U}$ & 2.7 \\
\hline SE-9 & .116341 & $12 / 17 / 2007$ & 0.31 & $\mathrm{U}$ & 0.31 & 154 & C & 6.2 & 36.4 & & 0.43 & 47.1 & C & 1.9 & 145 & $\mathrm{U}$ & 145 & 2.3 & U & 2.3 & 2.3 & u & 2.3 \\
\hline SE-10 & .116342 & $12 / 17 / 2007$ & 0.32 & $\mathrm{U}$ & 0.32 & 180 & $C$ & 6.4 & 49.7 & & 0.44 & 39.4 & C & 1.9 & 146 & $\mathrm{U}$ & 146 & 2.6 & \begin{tabular}{|l|l}
$\mathrm{U}$ \\
\end{tabular} & 2.6 & 2.6 & $\mathrm{U}$ & 2.6 \\
\hline $\mathrm{NW}-1$ & J16DT7 & \begin{tabular}{|l|}
$3 / 18 / 2008$ \\
\end{tabular} & 0.27 & $\mathrm{U}$ & 0.27 & 139 & & 5.4 & 53.7 & & 0.38 & 37.7 & & 1.6 & 141 & $\mathrm{U}$ & 141 & 2.4 & $\mathrm{U}$ & 2.4 & 3.5 & & 2.4 \\
\hline $\mathrm{NW}-2$ & J16DT8 & \begin{tabular}{|l|}
$3 / 18 / 2008$ \\
\end{tabular} & 0.28 & U & 0.28 & 132 & & 5.6 & 48.7 & & 0.39 & 38.8 & & 1.7 & 140 & $\mathrm{U}$ & 140 & 2.4 & $\mathrm{U}$ & 2.4 & 2.4 & $\mathrm{U}$ & 2.4 \\
\hline NW-3 & J16DT9 & \begin{tabular}{|c|}
$3 / 18 / 2008$ \\
\end{tabular} & 0.26 & $\mathrm{U}$ & 0.26 & 112 & & 5.2 & 35.6 & & 0.36 & 28.7 & & 1.6 & 133 & $\mathrm{U}$ & 133 & 2.2 & \begin{tabular}{|l|}
$\mathrm{u}$ \\
\end{tabular} & 2.2 & 2.2 & $\mathrm{U}$ & 2.2 \\
\hline NW-4 & J16DV0 & \begin{tabular}{|l|}
$3 / 18 / 2008$ \\
\end{tabular} & 0.26 & $\mathrm{U}$ & 0.26 & 131 & & 5.2 & 34.3 & & 0.36 & 27.7 & & 1.6 & 134 & $\mathrm{U}$ & 134 & 2.2 & $\mathrm{U}$ & 2.2 & 2.2 & $\mathrm{U}$ & 2.2 \\
\hline $\mathrm{NW}-5$ & J16DV1 & $3 / 18 / 2008$ & 0.26 & $\mathrm{U}$ & 0.26 & 174 & & 5.1 & 34.6 & & 0.36 & 28.1 & & 1.5 & 138 & $\mathrm{U}$ & 138 & 2.5 & \begin{tabular}{|l|} 
\\
\end{tabular} & 2.5 & 2.5 & U & 2.5 \\
\hline$N W-6$ & $\mathrm{~J} 16 \mathrm{DV} 2$ & \begin{tabular}{|l|}
$3 / 18 / 2008$ \\
\end{tabular} & 0.25 & $\mathrm{U}$ & 0.25 & 120 & & 5.1 & 40.6 & & 0.35 & 31.1 & & 1.5 & 134 & $\mathrm{U}$ & 134 & 2.4 & $\mathrm{U}$ & 2.4 & 2.4 & $\mathrm{U}$ & 2.4 \\
\hline NW-7 & $\mathrm{J16 \textrm {DV } 3}$ & $3 / 18 / 2008$ & 0.26 & $\mathrm{U}$ & 0.26 & 147 & & 5.3 & 67.7 & & 0.37 & 40.9 & & 1.6 & 134 & $\mathrm{U}$ & 134 & 2.5 & $\mathrm{U}$ & 2.5 & 2.5 & $\mathrm{U}$ & 2.5 \\
\hline$N W-8$ & $.116 \mathrm{DV} 4$ & \begin{tabular}{|l|}
$3 / 18 / 2008$ \\
\end{tabular} & 0.28 & $\mathrm{U}$ & 0.28 & 140 & & 5.5 & 55.2 & & 0.39 & 38.1 & & 1.7 & 144 & $\mathrm{U}$ & 144 & 2.6 & $\mathrm{U}$ & 2.6 & 7.6 & & 2.6 \\
\hline NW-9 & $.116 \mathrm{DV} 5$ & $3 / 18 / 2008$ & 0.28 & U & 0.28 & 112 & & 5.7 & 35.7 & & 0.40 & 29.4 & & 1.7 & 141 & $\mathrm{U}$ & 141 & 2.6 & $\mathrm{U}$ & 2.6 & 2.6 & $\mathrm{U}$ & 2.6 \\
\hline \multirow{2}{*}{\multicolumn{24}{|c|}{ Duplicate of }} \\
\hline & $.116 \mathrm{DV} 7$ & $3 / 18 / 2008$ & 0.29 & $\mathrm{U}$ & 0.29 & 126 & & 5.8 & 52.8 & & 0.40 & 41.9 & & 1.7 & 141 & $\mathrm{U}$ & 141 & 2.6 & $\mathrm{u}$ & 2.6 & 2.6 & U & 2.6 \\
\hline NW-10 & $316 \mathrm{DV} 6$ & \begin{tabular}{|l|}
$3 / 18 / 2008$ \\
\end{tabular} & 0.29 & $\mathrm{U}$ & 0.29 & 123 & & 5.8 & 54.0 & & 0.40 & 42.5 & & 1.7 & 143 & $\mathrm{U}$ & 143 & 2.7 & U & 2.7 & 2.7 & $\mathrm{U}$ & 2.7 \\
\hline Equip blank & $\mathrm{J} 16354$ & \begin{tabular}{|l|}
$12 / 17 / 2007$ \\
\end{tabular} & 0.09 & $\mathrm{U}$ & 0.09 & 9.2 & C & 1.9 & 0.21 & & 0.13 & 0.63 & \begin{tabular}{|c|} 
C \\
\end{tabular} & 0.56 & & & & & & & & & \\
\hline Equip blank & J19DT6 & $3 / 18 / 2008$ & 0.08 & $\mathrm{U}$ & 0.08 & 10.6 & & 1.6 & 0.11 & $\mathrm{U}$ & 0.11 & 0.48 & $\mathrm{U}$ & 0.48 & & & & 2.4 & U & 2.4 & 2.4 & $\mathrm{U}$ & 2.4 \\
\hline
\end{tabular}

Attachmen

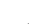

- 4 of 18

Originator H. M. Sulloway Date $\frac{04 / 03 / 08}{0403 / 08}$

Calc. No $\frac{\text { L. D. Habel }}{\text { O100F-CA-V0350 }}$ Rev. No. $\frac{04 / 03 / 08}{0}$ 
Attachment 1. 120-F-1 Verification Sampling Results.

\begin{tabular}{|c|c|c|c|c|c|c|c|c|c|c|c|c|c|c|c|c|c|c|c|c|c|c|c|}
\hline \multirow{2}{*}{$\begin{array}{c}\text { Sample } \\
\text { Location }\end{array}$} & \multirow{2}{*}{$\begin{array}{c}\text { HEIS } \\
\text { Number }\end{array}$} & \multirow{2}{*}{$\begin{array}{c}\text { Sample } \\
\text { Date }\end{array}$} & \multicolumn{3}{|c|}{ Cyanide } & \multicolumn{3}{|c|}{ Fluoride } & \multicolumn{3}{|c|}{ Nitrate } & \multicolumn{3}{|c|}{ Nitrite } & \multicolumn{3}{|c|}{ Phosphate } & \multicolumn{3}{|c|}{ Sulfate } & \multicolumn{3}{|c|}{ Sulfide } \\
\hline & & & $\mathrm{mg} / \mathrm{kg}$ & $Q$ & PQL & $\mathrm{mg} / \mathrm{kg}$ & $\mathrm{Q}$ & $\mathrm{PQL}$ & $\mathrm{mg} / \mathrm{kg}$ & Q & PQL & $\mathrm{mg} / \mathrm{kg}$ & Q & $\mathrm{PQL}$ & $\mathrm{mg} / \mathrm{kg}$ & $Q$ & PQL & $\mathrm{mg} / \mathrm{kg}$ & $Q$ & $\mathrm{PQL}$ & $\mathrm{mg} / \mathrm{kg}$ & $Q$ & $\mathrm{PQL}$ \\
\hline SE-1 & 116332 & $12 / 17 / 2007$ & 0.48 & $\mathrm{U}$ & 0.48 & 2.4 & $\overrightarrow{\mathrm{U}}$ & 2.4 & 2.37 & $\mathrm{U}$ & 2.4 & 2.37 & $\mathrm{U}$ & 2.4 & 2.4 & U & 2.4 & 2.4 & \begin{tabular}{|c|} 
\\
\end{tabular} & 2.4 & 15.4 & $\vec{U}$ & 15.4 \\
\hline SE-2 & $J 16333$ & $12 / 17 / 2007$ & 0.48 & $\mathrm{U}$ & 0.48 & 2.4 & $\mathrm{U}$ & 2.4 & 2.44 & $\mathrm{U}$ & 2.4 & 2.44 & $\mathrm{U}$ & 2.4 & 2.4 & $\mathrm{U}$ & 2.4 & 493 & D & 24 & 21.7 & $\mathrm{U}$ & 21.7 \\
\hline Duplicate of & & & & & & & & & & & & & & & & & & & & & & & \\
\hline$J 16333$ & $\mathrm{~J} 16334$ & $12 / 17 / 2007$ & 0.47 & $\mathrm{U}$ & 0.47 & 2.3 & $\mathrm{U}$ & 2.3 & 2.34 & $\mathrm{U}$ & 2.3 & 2.34 & $\mathrm{U}$ & 2.3 & 2.3 & $\mathrm{U}$ & 2.3 & 183 & D & 23 & 15.4 & u & 15.4 \\
\hline SE-3 & .116335 & $12 / 17 / 2007$ & 0.48 & $\mathrm{U}$ & 0.48 & 2.5 & $\mathrm{U}$ & 2.5 & 2.46 & $\mathrm{U}$ & 2.5 & 2.46 & $\mathrm{U}$ & 2.5 & 2.5 & $\mathrm{U}$ & 2.5 & 2.5 & $\mathrm{U}$ & 2.5 & 21.2 & $\mathrm{U}$ & 21.2 \\
\hline SE-4 & $J 16336$ & $12 / 17 / 2007$ & 0.5 & $\mathrm{U}$ & 0.5 & 3.9 & & 2.7 & 2.73 & $\mathrm{U}$ & 2.7 & 2.73 & $\mathrm{U}$ & 2.7 & 2.7 & $\mathrm{U}$ & 2.7 & 8410 & \begin{tabular}{|l|}
$\mathrm{D}$ \\
\end{tabular} & 270 & 23.7 & U & 23.7 \\
\hline SE-5 & $\mathrm{J} 16337$ & $12 / 17 / 2007$ & 0.51 & $\mathrm{U}$ & 0.51 & 3.4 & & 2.5 & 2.49 & $\mathrm{U}$ & 2.5 & 2.49 & $\mathrm{U}$ & 2.5 & 2.5 & $\mathrm{U}$ & 2.5 & 153 & \begin{tabular}{|l|} 
D \\
\end{tabular} & 2.5 & 21.5 & $\mathrm{U}$ & 21.5 \\
\hline SE-6 & $\mathrm{J} 16338$ & $12 / 17 / 2007$ & 0.5 & $\bar{U}$ & 0.5 & 2.5 & $\mathrm{U}$ & 2.5 & 2.54 & $\mathrm{U}$ & 2.5 & 2.54 & $\mathrm{U}$ & 2.5 & 2.5 & $\mathrm{U}$ & 2.5 & 2410 & \begin{tabular}{|l|}
$\mathrm{D}$ \\
\end{tabular} & 125 & 21.5 & $\bar{U}$ & 21.5 \\
\hline SE-7 & $\mathrm{J16339}$ & $12 / 17 / 2007$ & 0.5 & $\mathrm{U}$ & 0.5 & 2.6 & $U$ & 2.6 & 2.62 & $\mathrm{U}$ & 2.6 & 2.62 & $\mathrm{U}$ & 2.6 & 2.6 & $\bar{U}$ & 2.6 & 32.8 & & 2.6 & 21.5 & $\mathrm{U}$ & 21.5 \\
\hline SE-8 & $\mathrm{J} 16340$ & $12 / 17 / 2007$ & 0.49 & $\bar{U}$ & 0.49 & 2.7 & $\mathrm{U}$ & 2.7 & 2.72 & $\mathrm{U}$ & 2.7 & 2.72 & $\mathrm{U}$ & 2.7 & 2.7 & $\mathrm{U}$ & 2.7 & 11.4 & & 2.7 & 21.1 & $\mathrm{U}$ & 21.1 \\
\hline SE-9 & $\mathrm{J} 16341$ & $12 / 17 / 2007$ & 0.48 & $\mathrm{U}$ & 0.48 & 2.3 & $\mathrm{U}$ & 2.3 & 18.6 & & 2.3 & 2.27 & $\mathrm{U}$ & 2.3 & 6.8 & & 2.3 & 51.3 & & 2.3 & 22.3 & $\mathrm{U}$ & 22.3 \\
\hline$S E-10$ & $\mathrm{~J} 16342$ & $12 / 17 / 2007$ & 0.48 & $\mathrm{U}$ & 0.48 & 2.6 & $\mathrm{U}$ & 2.6 & 2.56 & $\mathrm{U}$ & 2.6 & 2.56 & $\mathrm{U}$ & 2.6 & 46.5 & & 2.6 & 3.2 & & 2.6 & 22.7 & $\mathrm{U}$ & 22.7 \\
\hline NW-1 & J16DT7 & $3 / 18 / 2008$ & & & & 2.4 & $\mathrm{U}$ & 2.4 & 3.53 & & 2.4 & 2.4 & $\mathrm{U}$ & 2.4 & 2.4 & $\mathrm{U}$ & 2.4 & 3.9 & & 2.4 & & & \\
\hline$N W-2$ & J16Dr8 & $3 / 18 / 2008$ & & & & 2.4 & $\mathrm{U}$ & 2.4 & 12.2 & & 2.36 & 2.36 & U & 2.36 & 2.4 & $\mathrm{U}$ & 2.4 & 3.9 & & 2.4 & & & \\
\hline NW-3 & J16DT9 & $3 / 18 / 2008$ & & & & 2.2 & $\mathrm{U}$ & 2.2 & 2.2 & $\mathrm{U}$ & 2.2 & 2.2 & U & 2.2 & 2.2 & $\mathrm{U}$ & 2.2 & 2.2 & \begin{tabular}{|l|}
0 \\
\end{tabular} & 2.2 & & & \\
\hline NW-4 & J16DV0 & $3 / 18 / 2008$ & & & & 2.2 & $\mathrm{U}$ & 2.2 & 2.24 & $\mathrm{U}$ & 2.24 & 2.24 & $\mathrm{U}$ & 2.24 & 2.2 & $\mathrm{U}$ & 2.2 & 4.0 & & 2.2 & & & \\
\hline NW-5 & J16DV1 & $3 / 18 / 2008$ & & & & 2.5 & $\mathrm{U}$ & 2.5 & 2.47 & $\mathrm{U}$ & 2.47 & 2.47 & U & 2.47 & 2.5 & U & 2.5 & 5.4 & & 2.5 & & & \\
\hline NW-6 & J16DV2 & $3 / 18 / 2008$ & & & & 2.4 & $\mathrm{U}$ & 2.4 & 2.36 & $\mathrm{U}$ & 2.36 & 2.36 & $\mathrm{U}$ & 2.36 & 2.4 & $\mathrm{U}$ & 2.4 & 3.3 & & 2.4 & & & \\
\hline NW-7 & $116 \mathrm{DV} 3$ & $3 / 18 / 2008$ & & & & 2.5 & U & 2.5 & 2.86 & & 2.48 & 2.48 & $\mathrm{U}$ & 2.48 & 2.5 & U & 2.5 & 2.5 & \begin{tabular}{|l|}
$\mathrm{U}$ \\
\end{tabular} & 2.5 & & & \\
\hline NW-8 & $\mathrm{J}_{16 \mathrm{DV} 4}$ & $3 / 18 / 2008$ & & & & 2.6 & $\mathrm{U}$ & 2.6 & 2.71 & & 2.57 & 2.57 & $\mathrm{U}$ & 2.57 & 2.6 & $\mathrm{U}$ & 2.6 & 13.4 & & 2.6 & & & \\
\hline NW-9 & J16DVS & $3 / 18 / 2008$ & & & & 2.6 & $\mathrm{U}$ & 2.6 & 3.54 & & 2.55 & 2.55 & $\mathrm{U}$ & 2.55 & 2.6 & $\mathrm{U}$ & 2.6 & 5.3 & & 2.6 & & & \\
\hline Duplicate of & & & & & & & & & & & & & & & & & & & & & & & \\
\hline $316 \mathrm{DV} 6$ & $116 \mathrm{DV} 7$ & $3 / 18 / 2008$ & & & & 2.6 & $U$ & 2.6 & 19.8 & & 2.64 & 2.64 & $\mathrm{u}$ & 2.64 & 2.6 & $\mathrm{U}$ & 2.6 & 4.3 & & 2.6 & & & \\
\hline $\mathrm{NW}-10$ & $.116 \mathrm{DV} 6$ & $3 / 18 / 2008$ & & & & 2.7 & $\mathrm{U}$ & 2.7 & 25.3 & & 2.68 & 2.68 & $\mathrm{U}$ & 2.68 & 2.7 & $\mathrm{U}$ & 2.7 & 5.5 & & 2.7 & & & \\
\hline Equip blank & $J 16354$ & $12 / 17 / 2007$ & & & & & & & & & & & & & & & & & & & & & \\
\hline Equip blank & I19DT6 & $3 / 18 / 2008$ & & & & 2.4 & $\mathrm{U}$ & 2.4 & 2.39 & $\mathrm{U}$ & 2.39 & 2.39 & $\mathrm{U}$ & 2.39 & 2.4 & $\mathrm{U}$ & 2.4 & 2.4 & U & 2.4 & & & \\
\hline
\end{tabular}

Attachment 1 Sheet No. 5 of 18 Originator H.M. Sulloway \& 4 Date

Calc. No. 0100 F-CA-V0350 Rev. No. $\frac{0}{0}$ 
Attachment 1, 120-F-1 Verification Sampling Results.

\begin{tabular}{|c|c|c|c|c|c|}
\hline Sample & HEIS & Sample & Hexaval & $\mathrm{nt} \mathrm{Cl}$ & omium \\
\hline Location & Number & Date & $\mathrm{mg} / \mathrm{kg}$ & $\mathrm{O}$ & PQL \\
\hline SE-1 & $\mathrm{J} 16 \mathrm{~B} 36$ & $2 / 19 / 2008$ & 0.21 & $\mathrm{U}$ & 0.21 \\
\hline SE-2 & $\mathrm{J16B37}$ & $2 / 19 / 2008$ & 0.21 & $\mathrm{U}$ & 0.21 \\
\hline SE-3 & $\mathrm{J16B38}$ & $2 / 19 / 2008$ & 0.21 & $\mathrm{U}$ & 0.21 \\
\hline SE-4 & $\mathrm{J16B39}$ & $2 / 19 / 2008$ & 1.2 & & 0.21 \\
\hline SE-5 & $316 B 40$ & $2 / 19 / 2008$ & 0.21 & $\mathrm{U}$ & 0.21 \\
\hline SE-6 & $\mathrm{J16B41}$ & $2 / 19 / 2008$ & 1.8 & & 0.21 \\
\hline SE-7 & $116 \mathrm{~B} 42$ & $2 / 19 / 2008$ & 0.20 & $\mathrm{U}$ & 0.20 \\
\hline SE-8 & J16B43 & $2 / 19 / 2008$ & 0.21 & $U$ & 0.21 \\
\hline SE-9 & $\mathrm{J16B44}$ & $2 / 19 / 2008$ & 0.21 & U & 0.21 \\
\hline SE-10 & J16B45 & $2 / 19 / 2008$ & 0.22 & $\mathrm{U}$ & 0.22 \\
\hline Duplicate of & & & & & \\
\hline $316 \mathrm{~B} 45$ & J16B46 & $2 / 19 / 2008$ & 0.21 & U & 0.21 \\
\hline NW-1 & J16DT7 & $3 / 18 / 2008$ & 0.21 & & 0.2 \\
\hline NW-2 & J16DT8 & $3 / 18 / 2008$ & 0.21 & U & 0.21 \\
\hline NW-3 & J16DT9 & $3 / 18 / 2008$ & 0.30 & & 0.2 \\
\hline NW-4 & J16DV0 & $3 / 18 / 2008$ & 0.2 & $\mathrm{U}$ & 0.2 \\
\hline NW-5 & $\mathrm{J} 16 \mathrm{DV} 1$ & $3 / 18 / 2008$ & 0.21 & $\mathrm{U}$ & 0.21 \\
\hline NW-6 & J16DV2 & $3 / 18 / 2008$ & 0.2 & $\mathrm{U}$ & 0.2 \\
\hline NW-7 & J16DV3 & $3 / 18 / 2008$ & 0.2 & U & 0.2 \\
\hline NW-8 & J16DV4 & $3 / 18 / 2008$ & 0.22 & $\mathrm{U}$ & 0.22 \\
\hline NW-9 & $116 \mathrm{DV} 5$ & $3 / 18 / 2008$ & 0.24 & & 0.21 \\
\hline plicate of & & & & & \\
\hline J16DV6 & $316 \mathrm{DV} 7$ & $3 / 18 / 2008$ & 0.22 & $u$ & 0.22 \\
\hline$N W-10$ & J16DV6 & $3 / 18 / 2008$ & 0.22 & $\mathrm{U}$ & 0.22 \\
\hline
\end{tabular}

Attachment

Sheet No. 6 of 18

Originator H.M. Sulloway WU/3 Date 04/03/08

Clecked L. D. Habel of Date 04/03/08

Calc. No. $0100 \mathrm{~F}-\mathrm{CA}-\mathrm{V} 0350$ Rev. No. 
Attachment 1. 120-F-1 Verification Sampling Results.

\begin{tabular}{|c|c|c|c|c|c|c|c|c|c|c|c|c|}
\hline \multirow[t]{2}{*}{ Constituent } & \multicolumn{3}{|c|}{$\begin{array}{l}\text { J16332 } \\
\text { Sample Location SE-1 } \\
\text { Sample Date } 12 / 17 / 07\end{array}$} & \multicolumn{3}{|c|}{$\begin{array}{c}\text { J16333 } \\
\text { Sample Location SE-2 } \\
\text { Sample Date } 12 / 17 / 07\end{array}$} & \multicolumn{3}{|c|}{$\begin{array}{c}\mathrm{J16334} \\
\text { Dup of } \mathrm{J} 16333 \\
\text { Sample Date } 12 / 17 / 07\end{array}$} & \multicolumn{3}{|c|}{$\begin{array}{l}\mathbf{J 1 6 3 3 5} \\
\text { Sample Location SE-3 } \\
\text { Sample Date 12/17/07 }\end{array}$} \\
\hline & $\mu \mathrm{g} / \mathrm{kg}$ & $Q$ & PQL & $\mu \mathrm{g} / \mathrm{kg}$ & Q & PQL & $\mu \mathrm{g} / \mathrm{kg}$ & $Q$ & PQL & $\mu \mathrm{g} / \mathrm{kg}$ & $Q$ & PQL \\
\hline \multicolumn{13}{|c|}{ Polychlorinated Biphenyls } \\
\hline Aroclor-1016 & 14 & $\mathrm{U}$ & 14 & 14 & $\mathrm{U}$ & 14 & 14 & $\mathrm{U}$ & 14 & 14 & U & 14 \\
\hline Aroclor-1221 & 14 & $\mathrm{U}$ & 14 & 14 & $\mathrm{U}$ & 14 & 14 & $U$ & 14 & 14 & U & 14 \\
\hline Aroclor -1232 & 14 & $\mathrm{U}$ & 14 & 14 & $\mathrm{U}$ & 14 & 14 & $\mathrm{U}$ & 14 & 14 & $U$ & 14 \\
\hline Aroclor -1242 & 14 & $\mathrm{U}$ & 14 & 14 & $\mathrm{U}$ & 14 & 14 & $\mathrm{U}$ & 14 & 14 & $\mathrm{U}$ & 14 \\
\hline Aroclor- 1248 & 14 & $\mathrm{U}$ & 14 & 14 & $\mathrm{U}$ & 14 & 14 & $\mathrm{U}$ & 14 & 14 & $\mathrm{U}$ & 14 \\
\hline Aroclor-1254 & 14 & $\mathrm{U}$ & 14 & 14 & $\mathrm{U}$ & 14 & 14 & $\mathrm{U}$ & 14 & 14 & $\mathrm{U}$ & 14 \\
\hline Aroclor -1260 & 14 & $\mathrm{U}$ & 14 & 14 & $\mathrm{U}$ & 14 & 14 & $\mathrm{U}$ & 14 & 14 & $\mathrm{U}$ & 14 \\
\hline \multicolumn{13}{|c|}{ Pesticides } \\
\hline Aldrin & 1.4 & $\mathrm{U}$ & 1.4 & 1.4 & $\mathrm{U}$ & 1.4 & 1.4 & $\mathrm{U}$ & 1.4 & 1.4 & $\mathrm{U}$ & 1.4 \\
\hline Alpha-BHC & 1.4 & $\mathrm{U}$ & 1.4 & 1.4 & $\mathrm{U}$ & 1.4 & 1.4 & $U$ & 1.4 & 1.4 & $\mathrm{U}$ & 1.4 \\
\hline Alpha-Chlordane & 1.4 & $\mathrm{U}$ & 1.4 & 1.4 & $\mathrm{U}$ & 1.4 & 1.4 & $\mathrm{U}$ & 1.4 & 1.4 & $\mathrm{U}$ & 1.4 \\
\hline Beta-BHC & 1.4 & $\mathrm{U}$ & 1.4 & 1.4 & $\mathrm{U}$ & 1.4 & 1.4 & $\mathrm{U}$ & 1.4 & 1.4 & $\mathrm{U}$ & 1.4 \\
\hline Delta-BHC & 1.4 & $\mathrm{U}$ & 1.4 & 1.4 & $\mathrm{U}$ & 1.4 & 1.4 & $\mathrm{U}$ & 1.4 & 1.4 & $\mathrm{U}$ & 1.4 \\
\hline Dichlorodiphenyldichloroethane & 1.4 & $\mathrm{U}$ & 1.4 & 1.4 & $\mathrm{U}$ & 1.4 & 1.4 & $\mathrm{U}$ & 1.4 & 1.4 & $\mathrm{U}$ & 1.4 \\
\hline Dichlorodiphenyldichloroethylene & 1.4 & $\mathrm{U}$ & 1.4 & 1.4 & $\mathrm{U}$ & 1.4 & 1.4 & $\mathrm{U}$ & 1.4 & 1.4 & $\mathrm{U}$ & 1.4 \\
\hline Dichlorodiphenyltrichloroethane & 1.4 & $\mathrm{U}$ & 1.4 & 1.4 & $\mathrm{U}$ & 1.4 & 2.1 & $I$ & 1.4 & 1.4 & $\mathrm{U}$ & 1.4 \\
\hline Dieldrin & 1.4 & $\mathrm{U}$ & 1.4 & 1.4 & $\mathrm{U}$ & 1.4 & 1.4 & $\mathrm{U}$ & 1.4 & 1.4 & $\mathrm{U}$ & 1.4 \\
\hline Endosulfan I & 1.4 & $\mathrm{U}$ & 1.4 & 1.4 & $\mathrm{U}$ & 1.4 & 1.4 & $\mathrm{U}$ & 1.4 & 1.4 & $\mathrm{U}$ & 1.4 \\
\hline Endosulfan II & 1.4 & $\mathrm{U}$ & 1.4 & 1.4 & $U$ & 1.4 & 1.4 & $\mathrm{U}$ & 1.4 & 1.4 & $\mathrm{U}$ & 1.4 \\
\hline Endosulfan sulfate & 1.4 & $\mathrm{U}$ & 1.4 & 1.4 & $\mathrm{U}$ & 1.4 & 1.4 & $\mathrm{U}$ & 1.4 & 1.4 & $\mathrm{U}$ & 1.4 \\
\hline Endrin & 1.4 & $\mathrm{U}$ & 1.4 & 1.4 & $\mathrm{U}$ & 1.4 & 1.4 & $\mathrm{U}$ & 1.4 & 1.4 & $\mathrm{U}$ & 1.4 \\
\hline Endrin aldehyde & 1.4 & $\bar{U}$ & 1.4 & 1.4 & $\mathrm{U}$ & 1.4 & 1.4 & $\mathrm{U}$ & 1.4 & 1.4 & $\mathrm{U}$ & 1.4 \\
\hline Endrin ketone & 1.4 & $\mathrm{U}$ & 1.4 & 1.4 & $\mathrm{U}$ & 1.4 & 1.4 & $\mathrm{U}$ & 1.4 & 1.4 & $\mathrm{U}$ & 1.4 \\
\hline Gamma-BHC (Lindane) & 1.4 & $\mathrm{U}$ & 1.4 & 1.4 & $\mathrm{U}$ & 1.4 & 1.4 & $\mathrm{U}$ & 1.4 & 1.4 & $\mathrm{U}$ & 1.4 \\
\hline gamma-Chlordane & 1.4 & $\mathrm{U}$ & 1.4 & 1.4 & $\mathrm{U}$ & 1.4 & 1.4 & $\mathrm{U}$ & 1.4 & 1.4 & $\mathrm{U}$ & 1.4 \\
\hline Heptachlor & 1.4 & $\mathrm{U}$ & 1.4 & 1.4 & $\mathrm{U}$ & 1.4 & 1.4 & $\mathrm{U}$ & 1.4 & 1.4 & $\mathrm{U}$ & 1.4 \\
\hline Heptachlor epoxide & 1.4 & $\mathrm{U}$ & 1.4 & 1.4 & $\mathrm{U}$ & 1.4 & 1.4 & $\mathrm{U}$ & 1.4 & 1.4 & $\mathrm{U}$ & 1.4 \\
\hline Methoxychlor & 1.4 & $\mathrm{U}$ & 1.4 & 1.4 & $\mathrm{U}$ & 1.4 & 1.4 & $U$ & 1.4 & 1.4 & $\mathrm{U}$ & 1.4 \\
\hline Toxaphene & 14 & $\mathrm{U}$ & 14 & 14 & $\mathrm{U}$ & 14 & 14 & $U$ & 14 & 14 & $\mathrm{U}$ & 14 \\
\hline \multicolumn{13}{|c|}{ Semivolatile Organic Analytes } \\
\hline 1,2,4-Trichlorobenzene & 340 & $\mathrm{U}$ & 340 & 350 & $\mathrm{U}$ & 350 & 350 & $\mathrm{U}$ & 350 & 340 & $\mathrm{U}$ & 340 \\
\hline 1,2-Dichlorobenzene & 340 & $\mathrm{U}$ & 340 & 350 & $\mathrm{U}$ & 350 & 350 & $\mathrm{U}$ & 350 & 340 & U & 340 \\
\hline 1,3-Dichlorobenzene & 340 & $\mathrm{U}$ & 340 & 350 & $\mathrm{U}$ & 350 & 350 & $\mathrm{U}$ & 350 & 340 & $\mathrm{U}$ & 340 \\
\hline 1,4-Dichlorobenzene & 340 & $\mathrm{U}$ & 340 & 350 & $\mathrm{U}$ & 350 & 350 & $\mathrm{U}$ & 350 & 340 & $U$ & 340 \\
\hline 2,4,5-Trichlorophenol & 860 & $\mathrm{U}$ & 860 & 860 & $\mathrm{U}$ & 860 & 860 & $\mathrm{U}$ & 860 & 860 & $\mathrm{U}$ & 860 \\
\hline 2,4,6-Trichlorophenol & 340 & $\mathrm{U}$ & 340 & 350 & $\mathrm{U}$ & 350 & 350 & $\mathrm{U}$ & 350 & 340 & $\mathrm{U}$ & 340 \\
\hline 2,4-Dichlorophenol & 340 & $\mathrm{U}$ & 340 & 350 & $\mathrm{U}$ & 350 & 350 & $\mathrm{U}$ & 350 & 340 & $\mathrm{U}$ & 340 \\
\hline 2,4-Dimethylphenol & 340 & $\mathrm{U}$ & 340 & 350 & $\mathrm{U}$ & 350 & 350 & $\mathrm{U}$ & 350 & 340 & $\mathrm{U}$ & 340 \\
\hline 2,4-Dinitrophenol & 860 & $\mathrm{U}$ & 860 & 860 & $\mathrm{U}$ & 860 & 860 & $\mathrm{U}$ & 860 & 860 & $\mathrm{U}$ & 860 \\
\hline 2,4-Dinitrotoluene & 340 & $\mathrm{U}$ & 340 & 350 & $\mathrm{U}$ & 350 & 350 & $\mathrm{U}$ & 350 & 340 & $\mathrm{U}$ & 340 \\
\hline 2,6-Dinitrotoluene & 340 & $\mathrm{U}$ & 340 & 350 & $\mathrm{U}$ & 350 & 350 & $\mathrm{U}$ & 350 & 340 & $\mathrm{U}$ & 340 \\
\hline 2-Chloronaphthalene & 340 & $U$ & 340 & 350 & $\mathrm{U}$ & 350 & 350 & $\mathrm{U}$ & 350 & 340 & $\mathrm{U}$ & 340 \\
\hline 2-Chlorophenol & 340 & $\mathrm{U}$ & 340 & 350 & $\mathrm{U}$ & 350 & 350 & $\mathrm{U}$ & 350 & 340 & $\mathrm{U}$ & 340 \\
\hline 2-Methylnaphthalene & 340 & $\mathrm{U}$ & 340 & 350 & $\mathrm{U}$ & 350 & 350 & $\mathrm{U}$ & 350 & 340 & $\mathrm{U}$ & 340 \\
\hline 2-Methylphenol (cresol, o-) & 340 & $U$ & 340 & 350 & $\mathrm{U}$ & 350 & 350 & $\mathrm{U}$ & 350 & 340 & $\mathrm{U}$ & 340 \\
\hline 2-Nitroaniline & 860 & $\mathrm{U}$ & 860 & 860 & $\mathrm{U}$ & 860 & 860 & $\mathrm{U}$ & 860 & 860 & $\mathrm{U}$ & 860 \\
\hline 2-Nitrophenol & 340 & $\mathrm{U}$ & 340 & 350 & $\mathrm{U}$ & 350 & 350 & $U$ & 350 & 340 & $\mathrm{U}$ & 340 \\
\hline & & & & & & Attachment & & 1 & & Sheet No. & & 0118 \\
\hline & & & & & & Originator & $\mathrm{H}$ & I. Sul & way $\mathrm{XI}$ & Date & & $03 / 08$ \\
\hline & & & & & & Checked & & D. H & & Date & & $03 / 08$ \\
\hline & & & & & & Calc. No. & 010 & $-\mathrm{CA}$ & 0350 & Rev. No. & & 0 \\
\hline
\end{tabular}


Attachment 1. 120-F-1 Verification Sampling Results.

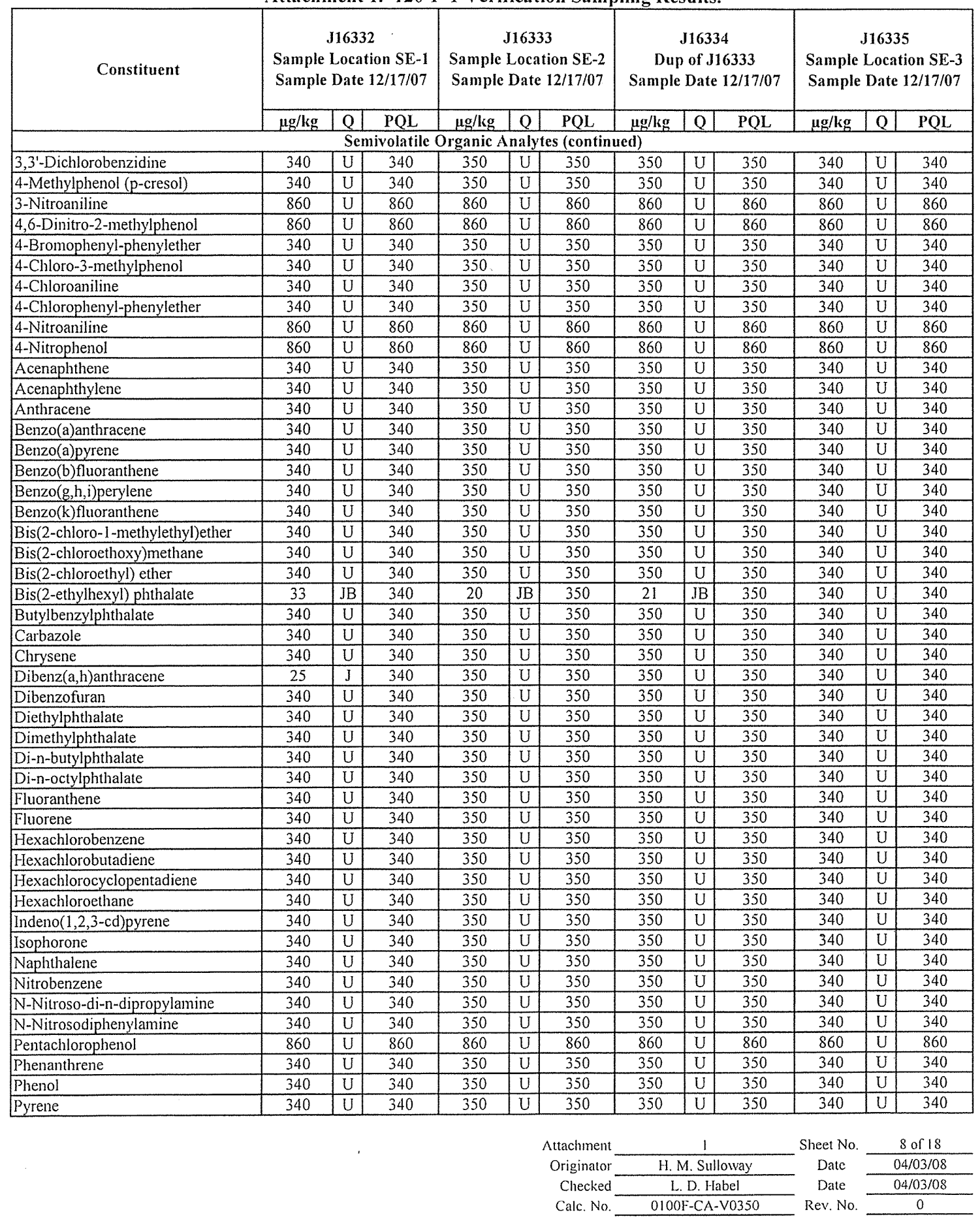


Attachment 1. 120-F-1 Verification Sampling Results.

\begin{tabular}{|c|c|c|c|c|c|c|c|c|c|c|c|c|}
\hline \multirow[t]{2}{*}{ Constituent } & \multicolumn{3}{|c|}{$\begin{array}{c}\text { J16336 } \\
\text { Sample Location SE-4 } \\
\text { Sample Date } 12 / 17 / 07\end{array}$} & \multicolumn{3}{|c|}{$\begin{array}{c}\text { J16337 } \\
\text { Sample Location SE-5 } \\
\text { Sample Date } 12 / 17 / 07\end{array}$} & \multicolumn{3}{|c|}{$\begin{array}{c}\text { J16338 } \\
\text { Sample Location SE-6 } \\
\text { Sample Date } 12 / 17 / 07\end{array}$} & \multicolumn{3}{|c|}{$\begin{array}{l}\mathrm{J16339} \\
\text { Sample Location SE- } \\
\text { Sample Date } 12 / 17 / 07\end{array}$} \\
\hline & $\mu \mathrm{g} / \mathrm{kg}$ & Q & $\mathrm{PQL}$ & $\mu \mathrm{g} / \mathrm{kg}$ & $Q$ & PQL & $\mu g / \mathrm{kg}$ & $Q$ & PQL & $\mu \mathrm{g} / \mathrm{kg}$ & $Q$ & $\mathrm{PQL}$ \\
\hline \multicolumn{13}{|c|}{ Polychlorinated Biphenyls } \\
\hline Aroclor -1016 & 14 & $\mathrm{U}$ & 14 & 14 & U & 14 & 14 & U & 14 & 14 & $\mathrm{U}$ & 14 \\
\hline Aroclor-1221 & 14 & $\mathrm{U}$ & 14 & 14 & U & 14 & 14 & U & 14 & 14 & $\mathrm{U}$ & 14 \\
\hline Aroclor-1232 & 14 & $\mathrm{U}$ & 14 & 14 & $\bar{U}$ & 14 & 14 & $\mathrm{U}$ & 14 & 14 & $\mathrm{U}$ & 14 \\
\hline Aroclor -1242 & 14 & $\mathrm{U}$ & 14 & 14 & $\mathrm{U}$ & 14 & 14 & $\mathrm{U}$ & 14 & 14 & U & 14 \\
\hline Aroclor-1248 & 14 & $\mathrm{U}$ & 14 & 14 & $\mathrm{U}$ & 14 & 14 & $\mathrm{U}$ & 14 & 14 & U & 14 \\
\hline Aroclor -1254 & 14 & $\mathrm{U}$ & 14 & 14 & $\mathrm{U}$ & 14 & 14 & $\mathrm{U}$ & 14 & 14 & $\mathrm{U}$ & 14 \\
\hline Aroclor-1260 & 14 & $\mathrm{U}$ & 14 & 14 & $\bar{U}$ & 14 & 14 & $\mathrm{U}$ & 14 & 14 & $\mathrm{U}$ & 14 \\
\hline \multicolumn{13}{|c|}{ Pesticides } \\
\hline Aldrin & 1.4 & $\mathrm{U}$ & 1.4 & 1.4 & $\mathrm{U}$ & 1.4 & 1.4 & $\mathrm{U}$ & 1.4 & 1.4 & $\mathrm{U}$ & 1.4 \\
\hline Alpha-BHC & 1.4 & U & 1.4 & 1.4 & U & 1.4 & 1.4 & $\mathrm{U}$ & 1.4 & 1.4 & $\mathrm{U}$ & 1.4 \\
\hline Alpha-Chlordane & 10 & & 1.4 & 1.4 & U & 1.4 & 8.6 & D & 1.4 & 1.4 & $\mathrm{U}$ & 1.4 \\
\hline Beta-BHC & 1.4 & U & 1.4 & 1.4 & $\mathrm{U}$ & 1.4 & 1.4 & U & 1.4 & 1.4 & U & 1.4 \\
\hline Delta-BHC & 1.4 & $\mathrm{U}$ & 1.4 & 1.4 & U & 1.4 & 1.4 & $\mathrm{U}$ & 1.4 & 1.4 & $\mathrm{U}$ & 1.4 \\
\hline Dichlorodiphenyldichloroethane & 1.4 & $\mathrm{U}$ & 1.4 & 1.4 & $\mathrm{U}$ & 1.4 & 1.4 & $\mathrm{U}$ & 1.4 & 1.4 & U & 1.4 \\
\hline Dichlorodiphenyldichloroethylene & 1.4 & $\mathrm{U}$ & 1.4 & 1.4 & $\mathrm{U}$ & 1.4 & 1.4 & $\mathrm{U}$ & 1.4 & 1.4 & $\mathrm{U}$ & 1.4 \\
\hline Dichlorodiphenyltrichloroethane & 1.4 & $\mathrm{U}$ & 1.4 & 1.4 & $\mathrm{U}$ & 1.4 & 1.4 & $\mathrm{U}$ & 1.4 & 1.4 & $\mathrm{U}$ & 1.4 \\
\hline Dieldrin & 1.4 & $\mathrm{U}$ & 1.4 & 1.4 & $\mathrm{U}$ & 1.4 & 1.4 & $\mathrm{U}$ & 1.4 & 1.4 & $\mathrm{U}$ & 1.4 \\
\hline Endosulfan I & 1.4 & U & 1.4 & 1.4 & $\mathrm{U}$ & 1.4 & 1.4 & $U$ & 1.4 & 1.4 & $\mathrm{U}$ & 1.4 \\
\hline Endosulfan II & 1.4 & $\mathrm{U}$ & 1.4 & 1.4 & $\mathrm{U}$ & 1.4 & 1.4 & U & 1.4 & 1.4 & U & 1.4 \\
\hline Endosulfan sulfate & 1.4 & $\mathrm{U}$ & 1.4 & 1.4 & $\mathrm{U}$ & 1.4 & 1.4 & U & 1.4 & 1.4 & $\mathrm{U}$ & 1.4 \\
\hline Endrin & 1.4 & $\mathrm{U}$ & 1.4 & 1.4 & $\mathrm{U}$ & 1.4 & 1.4 & $\mathrm{U}$ & 1.4 & 1.4 & U & 1.4 \\
\hline Endrin aldehyde & 1.4 & $\mathrm{U}$ & 1.4 & 1.4 & $\mathrm{U}$ & 1.4 & 1.4 & $\mathrm{U}$ & 1.4 & 1.4 & $\mathrm{U}$ & 1.4 \\
\hline Endrin ketone & 1.4 & $\mathrm{U}$ & 1.4 & 1.4 & $\mathrm{U}$ & 1.4 & 1.4 & $\mathrm{U}$ & 1.4 & 1.4 & $\mathrm{U}$ & 1.4 \\
\hline Gamma-BHC (Lindane) & 1.4 & $\mathrm{U}$ & 1.4 & 1.4 & $\mathrm{U}$ & 1.4 & 1.4 & $\mathrm{U}$ & 1.4 & 1.4 & $\mathrm{U}$ & 1.4 \\
\hline gamma-Chlordane & 13 & & 1.4 & 1.4 & U & 1.4 & 8.2 & $\mathrm{D}$ & 1.4 & 1.4 & $\mathrm{U}$ & 1.4 \\
\hline Heptachlor & 1.4 & $\mathrm{U}$ & 1.4 & 1.4 & $U$ & 1.4 & 1.4 & U & 1.4 & 1.4 & U & 1.4 \\
\hline Heptachlor epoxide & 1.4 & $\mathrm{U}$ & 1.4 & 1.4 & $\mathrm{U}$ & 1.4 & 1.4 & $\mathrm{U}$ & 1.4 & 1.4 & 0 & 1.4 \\
\hline Methoxychlor & 1.4 & $\mathrm{U}$ & 1.4 & 1.4 & U & 1.4 & 1.4 & $\mathrm{U}$ & 1.4 & 1.4 & $\mathrm{U}$ & 1.4 \\
\hline Toxaphene & 14 & U & 14 & 14 & $\mathrm{U}$ & 14 & 14 & $\mathrm{U}$ & 14 & 14 & U & 14 \\
\hline \multicolumn{13}{|c|}{ Semivolatile Organic Analytes } \\
\hline 1,2,4-Trichlorobenzene & 360 & U & 360 & 350 & $U$ & 350 & 350 & $\mathrm{U}$ & 350 & 350 & U & 350 \\
\hline 1,2-Dichlorobenzene & 360 & $\mathrm{U}$ & 360 & 350 & $\mathrm{U}$ & 350 & 350 & $\mathrm{U}$ & 350 & 350 & $\mathrm{U}$ & 350 \\
\hline 1,3-Dichlorobenzene & 360 & $\mathrm{U}$ & 360 & 350 & $\mathrm{U}$ & 350 & 350 & $\mathrm{U}$ & 350 & 350 & $\mathrm{U}$ & 350 \\
\hline 1,4-Dichlorobenzene & 360 & $\mathrm{U}$ & 360 & 350 & $\mathrm{U}$ & 350 & 350 & U & 350 & 350 & $\mathrm{U}$ & 350 \\
\hline $2,4,5$-Trichlorophenol & 890 & $\mathrm{U}$ & 890 & 870 & U & 870 & 880 & $\mathrm{U}$ & 880 & 870 & U & 870 \\
\hline 2,4,6-Trichlorophenol & 360 & $\mathrm{U}$ & 360 & 350 & U & 350 & 350 & $\mathrm{U}$ & 350 & 350 & $\mathrm{U}$ & 350 \\
\hline 2,4-Dichlorophenol & 360 & $\mathrm{U}$ & 360 & 350 & $\mathrm{U}$ & 350 & 350 & $\mathrm{U}$ & 350 & 350 & U & 350 \\
\hline 2,4-Dimethylphenol & 360 & $\mathrm{U}$ & 360 & 350 & $\mathrm{U}$ & 350 & 350 & $\mathrm{U}$ & 350 & 350 & $\mathrm{U}$ & 350 \\
\hline 2,4-Dinitrophenol & 890 & $\mathrm{U}$ & 890 & 870 & $\mathrm{U}$ & 870 & 880 & $\mathrm{U}$ & 880 & 870 & $\mathrm{U}$ & 870 \\
\hline 2,4-Dinitrotoluene & 360 & U & 360 & 350 & $\mathrm{U}$ & 350 & 350 & $\mathrm{U}$ & 350 & 350 & $\mathrm{U}$ & 350 \\
\hline 2,6-Dinitrotoluene & 360 & $U$ & 360 & 350 & $\mathrm{U}$ & 350 & 350 & $\mathrm{U}$ & 350 & 350 & $\mathrm{U}$ & 350 \\
\hline 2-Chloronaphthalene & 360 & $\mathrm{U}$ & 360 & 350 & $\mathrm{U}$ & 350 & 350 & U & 350 & 350 & $\mathrm{U}$ & 350 \\
\hline 2-Chlorophenol & 360 & U & 360 & 350 & $\mathrm{U}$ & 350 & 350 & $\mathrm{U}$ & 350 & 350 & U & 350 \\
\hline 2-Methylnaphthalene & 360 & $\mathrm{U}$ & 360 & 350 & $\mathrm{U}$ & 350 & 350 & $\mathrm{U}$ & 350 & 350 & $U$ & 350 \\
\hline 2-Methylphenol (cresol, o-) & 360 & $\mathrm{U}$ & 360 & 350 & U & 350 & 350 & U & 350 & 350 & $\mathrm{U}$ & 350 \\
\hline 2-Nitroaniline & 890 & $\mathrm{U}$ & 890 & 870 & $\mathrm{U}$ & 870 & 880 & $\mathrm{U}$ & 880 & 870 & $U$ & 870 \\
\hline 2-Nitrophenol & 360 & $\mathrm{U}$ & 360 & 350 & $\mathrm{UI}$ & 350 & 350 & $U$ & 350 & 350 & $U$ & 350 \\
\hline & & & & & & Attachment & & 1 & & Sheet No. & & 9 of 18 \\
\hline & & & & & & Originator & $\mathrm{H}$. & 1. Sul & Bway & Date & & $04 / 03 / 08$ \\
\hline & & & & & & Checked & & D. $\mathrm{H}$ & & Date & & $04 / 03 / 08$ \\
\hline & & & & & & Calc. No. & 0100 & $-\mathrm{CA}$ & 10350 & & & 0 \\
\hline
\end{tabular}


Attachment 1. 120-F-1 Verification Sampling Results.

\begin{tabular}{|c|c|c|c|c|c|c|c|c|c|c|c|c|}
\hline \multirow[t]{2}{*}{ Constituent } & \multicolumn{3}{|c|}{$\begin{array}{c}\text { J16336 } \\
\text { Sample Location SE-4 } \\
\text { Sample Date } 12 / 17 / 07\end{array}$} & \multicolumn{3}{|c|}{$\begin{array}{c}\text { J16337 } \\
\text { Sample Location SE-5 } \\
\text { Sample Date } 12 / 17 / 07\end{array}$} & \multicolumn{3}{|c|}{$\begin{array}{c}\text { J16338 } \\
\text { Sample Location SE-6 } \\
\text { Sample Date } 12 / 17 / 07\end{array}$} & \multicolumn{3}{|c|}{$\begin{array}{c}\text { J16339 } \\
\text { Sample Location SE-7 } \\
\text { Sample Date } 12 / 17 / 07\end{array}$} \\
\hline & $\mu \mathrm{g} / \mathrm{kg}$ & $\mathrm{Q}$ & PQL & $\mu \mathrm{g} / \mathrm{kg}$ & Q] & $\mathrm{PQL}$ & $\mu \mathrm{g} / \mathrm{kg}$ & $\mathrm{Q}$ & PQL & $\mu \mathrm{g} / \mathrm{kg}$ & $\mathbf{Q}$ & PQL \\
\hline \multicolumn{13}{|c|}{ Semivolatile Organic Analytes (continued) } \\
\hline 3,3'-Dichlorobenzidine & 360 & $\mathrm{U}$ & 360 & 350 & $\mathrm{U}$ & 350 & 350 & $\mathrm{U}$ & 350 & 350 & $\mathrm{U}$ & 350 \\
\hline 4-Methylphenol (p-cresol) & 360 & $\mathrm{U}$ & 360 & 350 & $\mathrm{U}$ & 350 & 350 & $\mathrm{U}$ & 350 & 350 & $\mathrm{U}$ & 350 \\
\hline 3-Nitroaniline & 890 & $\mathrm{U}$ & 890 & 870 & $\mathrm{U}$ & 870 & 880 & $\mathrm{U}$ & 880 & 870 & $\mathrm{U}$ & 870 \\
\hline 4,6-Dinitro-2-methylphenol & 890 & $\mathrm{U}$ & 890 & 870 & $\mathrm{U}$ & 870 & 880 & $\mathrm{U}$ & 880 & 870 & $\mathrm{U}$ & 870 \\
\hline 4-Bromophenyl-phenylether & 360 & $\mathrm{U}$ & 360 & 350 & $\mathrm{U}$ & 350 & 350 & $\mathrm{U}$ & 350 & 350 & $\mathrm{U}$ & 350 \\
\hline 4-Chloro-3-methylphenol & 360 & $\mathrm{U}$ & 360 & 350 & $\mathrm{U}$ & 350 & 350 & $\mathrm{U}$ & 350 & 350 & $\mathrm{U}$ & 350 \\
\hline 4-Chloroaniline & 360 & $\mathrm{U}$ & 360 & 350 & $\mathrm{U}$ & 350 & 350 & $U$ & 350 & 350 & $\mathrm{U}$ & 350 \\
\hline 4-Chlorophenyl-phenylether & 360 & $\mathrm{U}$ & 360 & 350 & $\mathrm{U}$ & 350 & 350 & $\mathrm{U}$ & 350 & 350 & $\mathrm{U}$ & 350 \\
\hline 4-Nitroaniline & 890 & $\mathrm{U}$ & 890 & 870 & $\mathrm{U}$ & 870 & 880 & U & 880 & 870 & $\mathrm{U}$ & 870 \\
\hline 4-Nitrophenol & 890 & U & 890 & 870 & $\mathrm{U}$ & 870 & 880 & $\mathrm{U}$ & 880 & 870 & $\mathrm{U}$ & 870 \\
\hline Acenaphthene & 360 & $\mathrm{U}$ & 360 & 350 & $\mathrm{U}$ & 350 & 350 & $\mathrm{U}$ & 350 & 350 & U & 350 \\
\hline Acenaphthylene & 360 & $\mathrm{U}$ & 360 & 350 & $\mathrm{U}$ & 350 & 350 & $\mathrm{U}$ & 350 & 350 & $\mathrm{U}$ & 350 \\
\hline Anthracene & 360 & $\mathrm{U}$ & 360 & 350 & $\mathrm{U}$ & 350 & 350 & $\mathrm{U}$ & 350 & 350 & U & 350 \\
\hline Benzo(a)anthracene & 360 & $\mathrm{U}$ & 360 & 350 & $\mathrm{U}$ & 350 & 350 & $U$ & 350 & 350 & $\mathrm{U}$ & 350 \\
\hline Benzo(a)pyrene & 360 & $\mathrm{U}$ & 360 & 350 & $\mathrm{U}$ & 350 & 350 & $\mathrm{U}$ & 350 & 350 & $\mathrm{U}$ & 350 \\
\hline Benzo(b)fluoranthene & 360 & $\mathrm{U}$ & 360 & 350 & $\mathrm{U}$ & 350 & 350 & $\mathrm{U}$ & 350 & 350 & $\mathrm{U}$ & 350 \\
\hline Benzo $(\mathrm{g}, \mathrm{h}, \mathrm{i})$ perylene & 360 & $\mathrm{U}$ & 360 & 350 & $\mathrm{U}$ & 350 & 350 & U & 350 & 350 & $\mathrm{U}$ & 350 \\
\hline Benzo(k)fluoranthene & 360 & $\mathrm{U}$ & 360 & 350 & $\mathrm{U}$ & 350 & 350 & $\mathrm{U}$ & 350 & 350 & $\mathrm{U}$ & 350 \\
\hline Bis(2-chloro-1-methylethyl)ether & 360 & $\mathrm{U}$ & 360 & 350 & $\mathrm{U}$ & 350 & 350 & $\mathrm{U}$ & 350 & 350 & U & 350 \\
\hline Bis(2-chloroethoxy)methane & 360 & $\mathrm{U}$ & 360 & 350 & $\mathrm{U}$ & 350 & 350 & $\mathrm{U}$ & 350 & 350 & U & 350 \\
\hline Bis(2-chloroethyl) ether & 360 & $\mathrm{U}$ & 360 & 350 & $\mathrm{U}$ & 350 & 350 & U & 350 & 350 & $\mathrm{U}$ & 350 \\
\hline Bis(2-ethylhexyl) phthalate & 22 & $\mathrm{JB}$ & 360 & 350 & $\mathrm{U}$ & 350 & 28 & 1 & 350 & 20 & $\mathrm{~J}$ & 350 \\
\hline Butylbenzylphthalate & 360 & $\mathrm{U}$ & 360 & 350 & $\mathrm{U}$ & 350 & 350 & $\mathrm{U}$ & 350 & 350 & $\mathrm{U}$ & 350 \\
\hline Carbazole & 360 & $\mathrm{U}$ & 360 & 350 & $\mathrm{U}$ & 350 & 350 & $\mathrm{U}$ & 350 & 350 & $\mathrm{U}$ & 350 \\
\hline Chrysene & 360 & $\mathrm{U}$ & 360 & 350 & $\mathrm{U}$ & 350 & 350 & $\mathrm{U}$ & 350 & 350 & $\mathrm{U}$ & 350 \\
\hline Dibenz $(\mathrm{a}, \mathrm{h})$ anthracene & 360 & $\mathrm{U}$ & 360 & 350 & $\mathrm{U}$ & 350 & 350 & U & 350 & 350 & U & 350 \\
\hline Dibenzofuran & 360 & $\mathrm{U}$ & 360 & 350 & $\mathrm{U}$ & 350 & 350 & $\mathrm{U}$ & 350 & 350 & $\mathrm{U}$ & 350 \\
\hline Diethylphthalate & 360 & $\mathrm{U}$ & 360 & 350 & $\mathrm{U}$ & 350 & 350 & $\mathrm{U}$ & 350 & 350 & U & 350 \\
\hline Dimethylphthalate & 360 & $\mathrm{U}$ & 360 & 350 & $\mathrm{U}$ & 350 & 350 & U & 350 & 350 & U & 350 \\
\hline Di-n-butylphthalate & 360 & $\mathrm{U}$ & 360 & 350 & $\mathrm{U}$ & 350 & 350 & $\mathrm{U}$ & 350 & 350 & $\mathrm{U}$ & 350 \\
\hline Di-n-octylphthalate & 360 & $\mathrm{U}$ & 360 & 350 & $\mathrm{U}$ & 350 & 350 & U & 350 & 350 & $\mathrm{U}$ & 350 \\
\hline Fluoranthene & 360 & $\mathrm{U}$ & 360 & 350 & $\mathrm{U}$ & 350 & 350 & $\mathrm{U}$ & 350 & 350 & $\mathrm{U}$ & 350 \\
\hline Fluorene & 360 & $\mathrm{U}$ & 360 & 350 & $\mathrm{U}$ & 350 & 350 & $\mathrm{U}$ & 350 & 350 & $\mathrm{U}$ & 350 \\
\hline Hexachlorobenzene & 360 & $\mathrm{U}$ & 360 & 350 & U & 350 & 350 & U & 350 & 350 & $\mathrm{U}$ & 350 \\
\hline Hexachlorobutadiene & 360 & $\mathrm{U}$ & 360 & 350 & $\mathrm{U}$ & 350 & 350 & U & 350 & 350 & $\mathrm{U}$ & 350 \\
\hline Hexachlorocyclopentadiene & 360 & $\mathrm{U}$ & 360 & 350 & $\mathrm{U}$ & 350 & 350 & $\mathrm{U}$ & 350 & 350 & U & 350 \\
\hline Hexachloroethane & 360 & $\mathrm{U}$ & 360 & 350 & $\mathrm{U}$ & 350 & 350 & $\mathrm{U}$ & 350 & 350 & $\mathrm{U}$ & -350 \\
\hline Indeno( $(1,2,3-c d)$ pyrene & 360 & $\mathrm{U}$ & 360 & 350 & $\mathrm{U}$ & 350 & 350 & $\mathrm{U}$ & 350 & 350 & $\mathrm{U}$ & 350 \\
\hline Isophorone & 360 & $\mathrm{U}$ & 360 & 350 & U & 350 & 350 & $\mathrm{U}$ & 350 & 350 & $\mathrm{U}$ & 350 \\
\hline Naphthalene & 360 & $\mathrm{U}$ & 360 & 350 & $\mathrm{U}$ & 350 & 350 & $\mathrm{U}$ & 350 & 350 & $\mathrm{U}$ & 350 \\
\hline Nitrobenzene & 360 & $\mathrm{U}$ & 360 & 350 & $\mathrm{U}$ & 350 & 350 & $\mathrm{U}$ & 350 & 350 & $\mathrm{U}$ & 350 \\
\hline N-Nitroso-di-n-dipropylamine & 360 & $\mathrm{U}$ & 360 & 350 & $\mathrm{U}$ & 350 & 350 & $\mathrm{U}$ & 350 & 350 & U & 350 \\
\hline N-Nitrosodiphenylamine & 360 & $\mathrm{U}$ & 360 & 350 & $\mathrm{U}$ & 350 & 350 & $\mathrm{U}$ & 350 & 350 & $\mathrm{U}$ & 350 \\
\hline Pentachlorophenol & 890 & $\mathrm{U}$ & 890 & 870 & U & 870 & 880 & $\mathrm{U}$ & 880 & 870 & $\mathrm{U}$ & 870 \\
\hline Phenanthrene & 360 & $\mathrm{U}$ & 360 & 350 & $\mathrm{U}$ & 350 & 350 & $\mathrm{U}$ & 350 & 350 & $\mathrm{U}$ & 350 \\
\hline Phenol & 360 & $\mathrm{U}$ & 360 & 350 & $U$ & 350 & 350 & $\mathrm{U}$ & 350 & 350 & U & 350 \\
\hline Pyrene & 360 & $\mathrm{U}$ & 360 & 350 & U & 350 & 350 & $\mathrm{U}$ & 350 & 350 & U & 350 \\
\hline & & & & & & Atlachment & & 1 & & Sheet No. & & 10 of 18 \\
\hline & & & & & & Originator & H. & Sul & way & Date & & $04 / 03 / 08$ \\
\hline & & & & & & Checked & & D. $\mathrm{H}$ & & Date & & $04 / 03 / 08$ \\
\hline & & & & & & Calc. No. & 0100 & $-\mathrm{CA}$ & 0350 & Rev. No. & & 0 \\
\hline
\end{tabular}


Attachment 1. 120-F-1 Verification Sampling Results.

\begin{tabular}{|c|c|c|c|c|c|c|c|c|c|c|c|c|}
\hline \multirow[t]{2}{*}{ Constituent } & \multicolumn{3}{|c|}{$\begin{array}{c}\text { J16340 } \\
\text { Sample Location SE-8 } \\
\text { Sample Date } 12 / 17 / 07\end{array}$} & \multicolumn{3}{|c|}{$\begin{array}{l}\text { J16341 } \\
\text { Sample Location SE-9 } \\
\text { Sample Date } 12 / 17 / 07\end{array}$} & \multicolumn{3}{|c|}{$\begin{array}{c}\mathrm{J16342} \\
\text { Sample Location SE-10 } \\
\text { Sample Date } 12 / 17 / 07\end{array}$} & \multicolumn{3}{|c|}{$\begin{array}{c}\text { J16DT7 } \\
\text { Sample Location NW-1 } \\
\text { Sample Date } 3 / 18 / 07\end{array}$} \\
\hline & $\mu \mathrm{g} / \mathrm{kg}$ & $Q$ & $\mathrm{PQL}$ & $\mu \mathrm{g} / \mathrm{kg}$ & $Q$ & PQL & $\mu \mathrm{g} / \mathrm{kg}$ & $Q$ & $\mathrm{PQL}$ & ug/kg & Q & PQL \\
\hline \multicolumn{13}{|c|}{ Polychlorinated Biphenyls } \\
\hline Aroclor-1016 & 14 & $\mathrm{U}$ & 14 & 15 & $\mathrm{U}$ & 15 & 15 & $\mathrm{U}$ & 15 & 14 & $\mathrm{U}$ & 14 \\
\hline Aroclor -1221 & 14 & $\mathrm{U}$ & 14 & 15 & $\mathrm{U}$ & 15 & 15 & $\mathrm{U}$ & 15 & 14 & $\mathrm{U}$ & 14 \\
\hline Aroclor-1232 & 14 & $\mathrm{U}$ & 14 & 15 & $\mathrm{U}$ & 15 & 15 & $\mathrm{U}$ & 15 & 14 & $\mathrm{U}$ & 14 \\
\hline Aroclor- 1242 & 14 & $\mathrm{U}$ & 14 & 15 & $\mathrm{U}$ & 15 & 15 & U & 15 & 14 & $\mathrm{U}$ & 14 \\
\hline Aroclor-1248 & 14 & $\mathrm{U}$ & 14 & 15 & $\mathrm{U}$ & 15 & 15 & U & 15 & 14 & $\mathrm{U}$ & 14 \\
\hline Aroclor-1254 & 14 & $\mathrm{U}$ & 14 & 23 & & 15 & 15 & $U$ & 15 & 14 & $\mathrm{U}$ & 14 \\
\hline Aroclor -1260 & 14 & $\mathrm{U}$ & 14 & 9.8 & $\mathrm{~J}$ & 15 & 15 & $\mathrm{U}$ & 15 & 14 & $\mathrm{U}$ & 14 \\
\hline \multicolumn{13}{|c|}{ Pesticides } \\
\hline Aldrin & 1.4 & UD & 1.4 & 1.5 & UD & 1.5 & 1.5 & UD & 1.5 & 1.4 & $\mathrm{U}$ & 1.4 \\
\hline Alpha-BHC & 1.4 & UD & 1.4 & 1.5 & UD & 1.5 & 1.5 & UD & 1.5 & 1.4 & $\mathrm{U}$ & 1.4 \\
\hline Alpha-Chlordane & 1,4 & UD & 1.4 & 1.5 & UD & 1.5 & 1.5 & UD & 1.5 & 1.4 & $\mathrm{U}$ & 1.4 \\
\hline Beta-BHC & 1.4 & UD & 1.4 & 1.5 & UD & 1.5 & 1.5 & UD & 1.5 & 1.4 & $\mathrm{U}$ & 1.4 \\
\hline Delta-BHC & 1.4 & UD & 1.4 & 1.5 & UD & 1.5 & 1.5 & UD & 1.5 & 1.4 & $\mathrm{U}$ & 1.4 \\
\hline Dichlorodiphenyldichloroethane & 1.4 & UD & 1.4 & 1.5 & UD & 1.5 & 1.5 & UD & 1.5 & 1.4 & $\mathrm{U}$ & 1.4 \\
\hline Dichlorodiphenyldichloroethylene & 1.4 & UD & 1.4 & 1.8 & JD & 1.5 & 1.5 & UD & 1.5 & 1.4 & $\mathrm{U}$ & 1.4 \\
\hline Dichlorodiphenyltrichloroethane & 1.4 & UD & 1.4 & 1.7 & JD & 1.5 & 1.5 & UD & 1.5 & 1.4 & $\mathrm{U}$ & 1.4 \\
\hline Dieldrin & 1.4 & UD & 1.4 & 1.5 & UD & 1.5 & 1.5 & UD & 1.5 & 1.4 & $\mathrm{U}$ & 1.4 \\
\hline Endosulfan I & 1.4 & UD & 1.4 & 1.8 & JD & 1.5 & 1.5 & UD & 1.5 & 1.4 & $\mathrm{U}$ & 1.4 \\
\hline Endosulfan II & 1.4 & UD & 1.4 & 1.5 & UD & 1.5 & 1.5 & UD & 1.5 & 1.4 & $\mathrm{U}$ & 1.4 \\
\hline Endosulfan sulfate & 1.4 & UD & 1.4 & 1.5 & UD & 1.5 & 1.5 & UD & 1.5 & 1.4 & $\mathrm{U}$ & 1.4 \\
\hline Endrin & 1.4 & UD & 1.4 & 1.5 & UD & 1.5 & 1.5 & UD & 1.5 & 1.4 & $\mathrm{U}$ & 1.4 \\
\hline Endrin aldehyde & 1.4 & UD & 1.4 & 1.5 & UD & 1.5 & 1.5 & UD & 1.5 & 1.4 & $\mathrm{U}$ & 1.4 \\
\hline Endrin ketone & 1.4 & UD & 1.4 & 1.5 & UD & 1.5 & 1.5 & UD & 1.5 & 1.4 & $\mathrm{U}$ & 1.4 \\
\hline Gamma-BHC (Lindane) & 1.4 & UD & 1.4 & 1.5 & UD & 1.5 & 1.5 & UD & 1.5 & 1.4 & $\mathrm{U}$ & 1.4 \\
\hline gamma-Chlordane & 1.4 & UD & 1.4 & 1.5 & UD & 1.5 & 1.5 & UD & 1.5 & 1.4 & $\mathrm{U}$ & 1.4 \\
\hline Heptachlor & 1.4 & UD & 1.4 & 1.5 & UD & 1.5 & 1.5 & UD & 1.5 & 1.4 & $\mathrm{U}$ & 1.4 \\
\hline Heptachlor epoxide & 1.4 & UD & 1.4 & 1.5 & UD & 1.5 & 1.5 & UD & 1.5 & 1.4 & U & 1.4 \\
\hline Methoxychlor & 1.4 & UD & 1.4 & 1.5 & UD & 1.5 & 1.5 & UD & 1.5 & 1.4 & $\mathrm{U}$ & 1.4 \\
\hline Toxaphene & 14 & UD & 14 & 15 & UD & 15 & 15 & UD & 15 & 14 & U & 14 \\
\hline \multicolumn{13}{|c|}{ Semivolatile Organic Analytes } \\
\hline 1,2,4-Trichlorobenzene & 350 & $\mathrm{U}$ & 350 & 360 & $\mathrm{U}$ & 360 & 370 & $\mathrm{U}$ & 370 & 340 & $\mathrm{U}$ & 340 \\
\hline 1,2-Dichlorobenzene & 350 & $\mathrm{U}$ & 350 & 360 & $\mathrm{U}$ & 360 & 370 & $\mathrm{U}$ & 370 & 340 & $\mathrm{U}$ & 340 \\
\hline 1,3-Dichlorobenzene & 350 & $\mathrm{U}$ & 350 & 360 & $\mathrm{U}$ & 360 & 370 & $\mathrm{U}$ & 370 & 340 & $\mathrm{U}$ & 340 \\
\hline 1,4-Dichlorobenzene & 350 & $\mathrm{U}$ & 350 & 360 & $\mathrm{U}$ & 360 & 370 & $\mathrm{U}$ & 370 & 340 & $\mathrm{U}$ & 340 \\
\hline $2,4,5$-Trichlorophenol & 870 & $\mathrm{U}$ & 870 & 910 & $\mathrm{U}$ & 910 & 920 & $\mathrm{U}$ & 920 & 850 & $\mathrm{U}$ & 850 \\
\hline 2,4,6-Trichlorophenol & 350 & $\mathrm{U}$ & 350 & 360 & $\mathrm{U}$ & 360 & 370 & U & 370 & 340 & $\mathrm{U}$ & 340 \\
\hline 2,4-Dichlorophenol & 350 & $\mathrm{U}$ & 350 & 360 & $\mathrm{U}$ & 360 & 370 & $\mathrm{U}$ & 370 & 340 & $\mathrm{U}$ & 340 \\
\hline 2,4-Dimethylphenol & 350 & $U$ & 350 & 360 & $\mathrm{U}$ & 360 & 370 & $\mathrm{U}$ & 370 & 340 & $\mathrm{U}$ & 340 \\
\hline 2,4-Dinitrophenol & 870 & $\mathrm{U}$ & 870 & 910 & $\mathrm{U}$ & 910 & 920 & $\mathrm{U}$ & 920 & 850 & U & 850 \\
\hline 2,4-Dinitrotoluene & 350 & $\bar{U}$ & 350 & 360 & $\mathrm{U}$ & 360 & 370 & $U$ & 370 & 350 & $\mathrm{U}$ & 350 \\
\hline 2,6-Dinitrotoluene & 350 & U & 350 & 360 & $\mathrm{U}$ & 360 & 370 & $U$ & 370 & 340 & U & 340 \\
\hline 2-Chloronaphthalene & 350 & $\mathrm{U}$ & 350 & 360 & $\mathrm{U}$ & 360 & 370 & $\mathrm{U}$ & 370 & 340 & $\mathrm{U}$ & 340 \\
\hline 2-Chlorophenol & 350 & $\mathrm{U}$ & 350 & 360 & $\mathrm{U}$ & 360 & 370 & $\mathrm{U}$ & 370 & 340 & U & 340 \\
\hline 2-Methylnaphthalene & 350 & $\mathrm{U}$ & 350 & 360 & $\mathrm{U}$ & 360 & 370 & $\mathrm{U}$ & 370 & 340 & U & 340 \\
\hline 2-Methylphenol (cresol, o-) & 350 & U & 350 & 360 & $\mathrm{U}$ & 360 & 370 & U & 370 & 340 & U & 340 \\
\hline 2-Nitroaniline & 870 & $\mathrm{U}$ & 870 & 910 & $\mathrm{U}$ & 910 & 920 & $\mathrm{U}$ & 920 & 850 & $\mathrm{U}$ & 850 \\
\hline 2-Nitrophenol & 350 & $\mathrm{U}$ & 350 & 360 & $\mathrm{U}$ & 360 & 370 & $\mathrm{U}$ & 370 & 340 & $U$ & 340 \\
\hline & & & & & & Attachment & & 1 & & Sheet No. & & 11 of 18 \\
\hline & & & & & & Originator & H. & 4. Sull & & Date & & $4 / 03 / 08$ \\
\hline & & & & & & Checked & & D. Ha & & Date & & $4 / 03 / 08$ \\
\hline & & & & & & Calc. No & 0100 & $\mathrm{FCA}-$ & 350 & & & 0 \\
\hline
\end{tabular}


Attachment 1. 120-F-1 Verification Sampling Results.

\begin{tabular}{|c|c|c|c|c|c|c|c|c|c|c|c|c|}
\hline \multirow[t]{2}{*}{ Constituent } & \multicolumn{3}{|c|}{$\begin{array}{c}\text { J16340 } \\
\text { Sample Location SE-8 } \\
\text { Sample Date } 12 / 17 / 07\end{array}$} & \multicolumn{3}{|c|}{$\begin{array}{c}\text { J16341 } \\
\text { Sample Location SE-9 } \\
\text { Sample Date } 12 / 17 / 07\end{array}$} & \multicolumn{3}{|c|}{$\begin{array}{c}\mathrm{J16342} \\
\text { Sample Location SE-10 } \\
\text { Sample Date } 12 / 17 / 07\end{array}$} & \multicolumn{3}{|c|}{$\begin{array}{c}\text { J16DT7 } \\
\text { Sample Location NW-1 } \\
\text { Sample Date 3/18/07 }\end{array}$} \\
\hline & $\mu \mathrm{g} / \mathrm{kg}$ & Q & PQL & $\mu \mathrm{g} / \mathrm{kg}$ & $Q$ & PQL & $\mu \mathrm{g} / \mathrm{kg}$ & $Q$ & PQL & $\mu \mathrm{g} / \mathrm{kg}$ & Q & PQL \\
\hline \multicolumn{13}{|c|}{ Semivolatile Organic Analytes (continued) } \\
\hline 3,3'-Dichlorobenzidine & 350 & $\mathrm{U}$ & 350 & 360 & $\mathrm{U}$ & 360 & 370 & $\mathrm{U}$ & 370 & 340 & U & 340 \\
\hline 4-Methylphenol ( $\mathrm{p}$-cresol) & 350 & $\mathrm{U}$ & 350 & 360 & $\mathrm{U}$ & 360 & 370 & U & 370 & 340 & $\mathrm{U}$ & 340 \\
\hline 3-Nitroaniline & 870 & $\mathrm{U}$ & 870 & 910 & $\mathrm{U}$ & 910 & 920 & $\mathrm{U}$ & 920 & 850 & U & 850 \\
\hline 4,6-Dinitro-2-methylphenol & 870 & $\mathrm{U}$ & 870 & 910 & $\mathrm{U}$ & 910 & 920 & $\mathrm{U}$ & 920 & 850 & $\mathrm{U}$ & 850 \\
\hline 4-Bromophenyl-phenylether & 350 & $\mathrm{U}$ & 350 & 360 & $\mathrm{U}$ & 360 & 370 & $\mathrm{U}$ & 370 & 340 & $\mathrm{U}$ & 340 \\
\hline 4-Chloro-3-methylphenol & 350 & $\mathrm{U}$ & 350 & 360 & $\mathrm{U}$ & 360 & 370 & $\mathrm{U}$ & 370 & 340 & $\mathrm{U}$ & 340 \\
\hline 4-Chloroaniline & 350 & $\mathrm{U}$ & 350 & 360 & $\mathrm{U}$ & 360 & 370 & $\mathrm{U}$ & 370 & 340 & $\mathrm{U}$ & 340 \\
\hline 4-Chlorophenyl-phenylether & 350 & $\mathrm{U}$ & 350 & 360 & $\mathrm{U}$ & 360 & 370 & $\mathrm{U}$ & 370 & 340 & $\mathrm{U}$ & 340 \\
\hline 4-Nitroaniline & 870 & $\mathrm{U}$ & 870 & 910 & $\mathrm{U}$ & 910 & 920 & $\mathrm{U}$ & 920 & 850 & $\mathrm{U}$ & 850 \\
\hline 4-Nitrophenol & 870 & $\mathrm{U}$ & 870 & 910 & $\mathrm{U}$ & 910 & 920 & $\mathrm{U}$ & 920 & 850 & $\mathrm{U}$ & 850 \\
\hline Acenaphthene & 350 & $\mathrm{U}$ & 350 & 360 & $U$ & 360 & 370 & $\mathrm{U}$ & 370 & 340 & $\mathrm{U}$ & 340 \\
\hline Acenaphthylene & 350 & $\mathrm{U}$ & 350 & 360 & U & 360 & 370 & U & 370 & 340 & $\mathrm{U}$ & 340 \\
\hline Anthracene & 350 & $\bar{U}$ & 350 & 360 & $\mathrm{U}$ & 360 & 370 & $\mathrm{U}$ & 370 & 340 & $\mathrm{U}$ & 340 \\
\hline Benzo(a)anthracene & 350 & $\mathrm{U}$ & 350 & 360 & $\mathrm{U}$ & 360 & 370 & $\mathrm{U}$ & 370 & 340 & $\mathrm{U}$ & 340 \\
\hline Benzo(a)pyrene & 350 & $\mathrm{U}$ & 350 & 360 & $\mathrm{U}$ & 360 & 370 & U & 370 & 340 & $\mathrm{U}$ & 340 \\
\hline Benzo(b)fluoranthene & 350 & $\mathrm{U}$ & 350 & 360 & $\mathrm{U}$ & 360 & 370 & $\mathrm{U}$ & 370 & 340 & $\mathrm{U}$ & 340 \\
\hline Benzo(g,h,i)perylene & 350 & $\mathrm{U}$ & 350 & 360 & $\mathrm{U}$ & 360 & 370 & $\mathrm{U}$ & 370 & 340 & $\mathrm{U}$ & 340 \\
\hline Benzo(k)fluoranthene & 350 & $\mathrm{U}$ & 350 & 360 & $\mathrm{U}$ & 360 & 370 & $\mathrm{U}$ & 370 & 340 & $\mathrm{U}$ & 340 \\
\hline Bis(2-chloro-1-methylethyl)ether & 350 & $\mathrm{U}$ & 350 & 360 & $U$ & 360 & 370 & $\mathrm{U}$ & 370 & 340 & $\mathrm{U}$ & 340 \\
\hline Bis(2-chloroethoxy)methane & 350 & $\mathrm{U}$ & 350 & 360 & $\mathrm{U}$ & 360 & 370 & $\mathrm{U}$ & 370 & 340 & $\mathrm{U}$ & 340 \\
\hline Bis(2-chloroethyl) ether & 350 & $\mathrm{U}$ & 350 & 360 & $\mathrm{U}$ & 360 & 370 & $\mathrm{U}$ & 370 & 340 & $\mathrm{U}$ & 340 \\
\hline Bis(2-ethylhexyl) phthalate & 200 & $\mathrm{~J}$ & 350 & 27 & $\mathrm{~J}$ & 360 & 25 & $\mathrm{~J}$ & 370 & 25 & $\mathrm{JB}$ & 350 \\
\hline Butylbenzylphthalate & 350 & $\mathrm{U}$ & 350 & 360 & $\mathrm{U}$ & 360 & 370 & $\mathrm{U}$ & 370 & 340 & $\mathrm{U}$ & 340 \\
\hline Carbazole & 350 & $\mathrm{U}$ & 350 & 360 & $\mathrm{U}$ & 360 & 370 & $\mathrm{U}$ & 370 & 340 & $\mathrm{U}$ & 340 \\
\hline Chrysene & 350 & $\mathrm{U}$ & 350 & 360 & $\mathrm{U}$ & 360 & 370 & $\mathrm{U}$ & 370 & 340 & $\mathrm{U}$ & 340 \\
\hline Dibenz $(a, h)$ anthracene & 350 & $\mathrm{U}$ & 350 & 360 & $\mathrm{U}$ & 360 & 370 & $\mathrm{U}$ & 370 & 340 & $\mathrm{U}$ & 340 \\
\hline Dibenzofuran & 350 & $\mathrm{U}$ & 350 & 360 & $\mathrm{U}$ & 360 & 370 & $\mathrm{U}$ & 370 & 340 & $\mathrm{U}$ & 340 \\
\hline Diethylphthalate & 350 & $\mathrm{U}$ & 350 & 360 & $\mathrm{U}$ & 360 & 370 & $\mathrm{U}$ & 370 & 340 & $\mathrm{U}$ & 340 \\
\hline Dimethylphthalate & 350 & $\mathrm{U}$ & 350 & 360 & $\mathrm{U}$ & 360 & 370 & $\mathrm{U}$ & 370 & 340 & $\mathrm{U}$ & 340 \\
\hline Di-n-butylphthalate & 350 & $\mathrm{U}$ & 350 & 360 & $\mathrm{U}$ & 360 & 370 & $\mathrm{U}$ & 370 & 340 & $\mathrm{U}$ & 340 \\
\hline Di-n-octylphthalate & 350 & $\mathrm{U}$ & 350 & 360 & $\mathrm{U}$ & 360 & 370 & U & 370 & 340 & $\mathrm{U}$ & 340 \\
\hline Fluoranthene & 350 & $\mathrm{U}$ & 350 & 360 & U & 360 & 370 & U & 370 & 340 & $U$ & 340 \\
\hline Fluorene & 350 & $\mathrm{U}$ & 350 & 360 & $\mathrm{U}$ & 360 & 370 & $\mathrm{U}$ & 370 & 340 & $\mathrm{U}$ & 340 \\
\hline Hexachlorobenzene & 350 & $\mathrm{U}$ & 350 & 360 & $\mathrm{U}$ & 360 & 370 & $\mathrm{U}$ & 370 & 340 & $\mathrm{U}$ & 340 \\
\hline Hexachlorobutadiene & 350 & $\mathrm{U}$ & 350 & 360 & $\mathrm{U}$ & 360 & 370 & $\mathrm{U}$ & 370 & 340 & $\mathrm{U}$ & 340 \\
\hline Hexachlorocyclopentadiene & 350 & $\mathrm{U}$ & 350 & 360 & $\mathrm{U}$ & 360 & 370 & $\mathrm{U}$ & 370 & 340 & $\mathrm{U}$ & 340 \\
\hline Hexachloroethane & 350 & U & 350 & 360 & $\mathrm{U}$ & 360 & 370 & U & 370 & 340 & $\mathrm{U}$ & 340 \\
\hline Indeno(1,2,3-cd)pyrene & 350 & $\mathrm{U}$ & 350 & 360 & $\mathrm{U}$ & 360 & 370 & $\mathrm{U}$ & 370 & 340 & $\mathrm{U}$ & 340 \\
\hline Isophorone & 350 & $\mathrm{U}$ & 350 & 360 & $\mathrm{U}$ & 360 & 370 & $\mathrm{U}$ & 370 & 340 & $\mathrm{U}$ & 340 \\
\hline Naphthalene & 350 & $\mathrm{U}$ & 350 & 360 & U & 360 & 370 & $\mathrm{U}$ & 370 & 340 & $\mathrm{U}$ & 340 \\
\hline Nitrobenzene & 350 & $\mathrm{U}$ & 350 & 360 & $\mathrm{U}$ & 360 & 370 & $\mathrm{U}$ & 370 & 340 & $\mathrm{U}$ & 340 \\
\hline N-Nitroso-di-n-dipropylamine & 350 & $\mathrm{U}$ & 350 & 360 & U & 360 & 370 & $\mathrm{U}$ & 370 & 340 & $\mathrm{U}$ & 340 \\
\hline N-Nitrosodiphenylamine & 350 & $\mathrm{U}$ & 350 & 360 & $U$ & 360 & 370 & U & 370 & 340 & $\mathrm{U}$ & 340 \\
\hline Pentachlorophenol & 870 & $\mathrm{U}$ & 870 & 910 & $U$ & 910 & 920 & $\mathrm{U}$ & 920 & 850 & $\mathrm{U}$ & 850 \\
\hline Phenanthrene & 350 & $\mathrm{U}$ & 350 & 360 & U & 360 & 370 & $\mathrm{U}$ & 370 & 340 & $\mathrm{U}$ & 340 \\
\hline Phenol & 350 & U & 350 & 360 & $\mathrm{U}$ & 360 & 370 & $\mathrm{U}$ & 370 & 340 & $\mathrm{U}$ & 340 \\
\hline Pyrene & 350 & $\mathrm{U}$ & 350 & 360 & $U$ & 360 & 370 & $U$ & 370 & 340 & $U$ & 340 \\
\hline & & & & & & Attachment & & 1 & & Sheet No. & & 2 of 18 \\
\hline & & & & & & Originator & & Sul & & Date & & $4 / 03 / 08$ \\
\hline & & & & & & Checked & & D. H & & Date & & $4 / 03 / 08$ \\
\hline & & & & & & Calc No. & 010 & $-\mathrm{CA}$ & 350 & Rev. No. & & 0 \\
\hline
\end{tabular}


Attachment 1. 120-F-1 Verification Sampling Results.

\begin{tabular}{|c|c|c|c|c|c|c|c|c|c|c|c|c|}
\hline \multirow[t]{2}{*}{ Constituent } & \multicolumn{3}{|c|}{$\begin{array}{c}\text { J16DT8 } \\
\text { Sample Location NW-2 } \\
\text { Sample Date 3/18/07 }\end{array}$} & \multicolumn{3}{|c|}{$\begin{array}{c}\text { J16DT9 } \\
\text { Sample Location NW-3 } \\
\text { Sample Date } 3 / 18 / 07\end{array}$} & \multicolumn{3}{|c|}{$\begin{array}{c}\text { J16DV0 } \\
\text { Sample Location NW }-4 \\
\text { Sample Date } 3 / 18 / 07\end{array}$} & \multicolumn{3}{|c|}{$\begin{array}{c}\text { J16DV1 } \\
\text { Sample Location NW-5 } \\
\text { Sample Date 3/18/07 }\end{array}$} \\
\hline & $\mu \mathrm{g} / \mathrm{kg}$ & $Q$ & PQL & $\mu \mathrm{g} / \mathrm{kg}$ & $Q$ & $\mathrm{PQL}$ & $\mu \mathrm{g} / \mathrm{kg}$ & $\mathrm{Q}$ & PQL & $\mu \mathrm{gg} / \mathrm{kg}$ & $Q$ & PQL \\
\hline \multicolumn{13}{|c|}{ Polychlorinated Biphenyls } \\
\hline Aroclor-1016 & 14 & $\mathrm{U}$ & 14 & 14 & $\mathrm{U}$ & 14 & 13 & $\mathrm{U}$ & 13 & 14 & $\mathrm{U}$ & 14 \\
\hline Aroclor-1221 & 14 & $\mathrm{U}$ & 14 & 14 & $\mathrm{U}$ & 14 & 13 & $\mathrm{U}$ & 13 & 14 & $\mathrm{U}$ & 14 \\
\hline Aroclor-1232 & 14 & $\mathrm{U}$ & 14 & 14 & $\mathrm{U}$ & 14 & 13 & $\mathrm{U}$ & 13 & 14 & $\mathrm{U}$ & 14 \\
\hline Aroclor- 1242 & 14 & $\mathrm{U}$ & 14 & 14 & $\mathrm{U}$ & 14 & 13 & $\mathrm{U}$ & 13 & 14 & $\mathrm{U}$ & 14 \\
\hline Aroclor-1248 & 14 & $U$ & 14 & 14 & $\mathrm{U}$ & 14 & 13 & $\mathrm{U}$ & 13 & 14 & $\mathrm{U}$ & 14 \\
\hline Aroclor -1254 & 14 & $\mathrm{U}$ & 14 & 14 & $\mathrm{U}$ & 14 & 13 & $\mathrm{U}$ & 13 & 14 & $\mathrm{U}$ & 14 \\
\hline Aroclor- 1260 & 14 & $\mathrm{U}$ & 14 & 14 & $\mathrm{U}$ & 14 & 13 & $\mathrm{U}$ & 13 & 14 & $\mathrm{U}$ & 14 \\
\hline \multicolumn{13}{|c|}{ Pesticides } \\
\hline Aldrin & 1.4 & $\mathrm{U}$ & 1.4 & 1.4 & $\mathrm{U}$ & 1.4 & 1.3 & $\mathrm{U}$ & 1.3 & 1.4 & $\mathrm{U}$ & 1.4 \\
\hline Alpha-BHC & 1.4 & $\mathrm{U}$ & 1.4 & 1.4 & $\mathrm{U}$ & 1.4 & 1.3 & $\mathrm{U}$ & 1.3 & 1.4 & $\mathrm{U}$ & 1.4 \\
\hline Alpha-Chlordane & 1.6 & $\mathrm{~J}$ & 1.4 & 1.4 & $\mathrm{U}$ & 1.4 & 1.3 & $\mathrm{U}$ & 1.3 & 1.4 & $\mathrm{U}$ & 1.4 \\
\hline Beta-BHC & 1.4 & $\mathrm{U}$ & 1.4 & 1.4 & $\mathrm{U}$ & 1.4 & 1.3 & $\mathrm{U}$ & 1.3 & 1.4 & $\mathrm{U}$ & 1.4 \\
\hline Delta-BHC & 1.4 & $\mathrm{U}$ & 1.4 & 1.4 & $\mathrm{U}$ & 1.4 & 1.3 & $\mathrm{U}$ & 1.3 & 1.4 & $\mathrm{U}$ & 1.4 \\
\hline Dichlorodiphenyldichloroethane & 1.4 & $U$ & 1.4 & 1.4 & $\mathrm{U}$ & 1.4 & 1.3 & $\mathrm{U}$ & 1.3 & 1.4 & $\mathrm{U}$ & 1.4 \\
\hline Dichlorodiphenyldichloroethylene & 1.4 & $\mathrm{U}$ & 1.4 & 1.4 & $\mathrm{U}$ & 1.4 & 1.3 & $\mathrm{U}$ & 1.3 & 1.4 & $\mathrm{U}$ & 1.4 \\
\hline Dichlorodiphenyltrichloroethane & 1.4 & $\mathrm{U}$ & 1.4 & 1.4 & $\mathrm{U}$ & 1.4 & 1.3 & $\mathrm{U}$ & 1.3 & 1.4 & $\mathrm{U}$ & 1.4 \\
\hline Dieldrin & 1.4 & $\mathrm{U}$ & 1.4 & 1.4 & $\mathrm{U}$ & 1.4 & 1.3 & $\mathrm{U}$ & 1.3 & 1.4 & $\mathrm{U}$ & 1.4 \\
\hline Endosulfan I & 1.4 & $\mathrm{U}$ & 1.4 & 1.4 & $\mathrm{U}$ & 1.4 & 1.3 & $\mathrm{U}$ & 1.3 & 1.4 & $\mathrm{U}$ & 1.4 \\
\hline Endosulfan II & 1.4 & $\mathrm{U}$ & 1.4 & 1.4 & $\mathrm{U}$ & 1.4 & 1.3 & $\mathrm{U}$ & 1.3 & 1.4 & $\mathrm{U}$ & 1.4 \\
\hline Endosulfan sulfate & 1.4 & $\mathrm{U}$ & 1.4 & 1.4 & $\mathrm{U}$ & 1.4 & 1.3 & $\mathrm{U}$ & 1.3 & 1.4 & $\mathrm{U}$ & 1.4 \\
\hline Endrin & 1.4 & $\mathrm{U}$ & 1.4 & 1.4 & $\mathrm{U}$ & 1.4 & 1.3 & $\mathrm{U}$ & 1.3 & 1.4 & $\mathrm{U}$ & 1.4 \\
\hline Endrin aldehyde & 1.4 & $\mathrm{U}$ & 1.4 & 1.4 & $\mathrm{U}$ & 1.4 & 1.3 & $\mathrm{U}$ & 1.3 & 1.4 & $\mathrm{U}$ & 1.4 \\
\hline Endrin ketone & 1.4 & $\mathrm{U}$ & 1.4 & 1.4 & $\mathrm{U}$ & 1.4 & 1.3 & $\mathrm{U}$ & 1.3 & 1.4 & $\mathrm{U}$ & 1.4 \\
\hline Gamma-BHC (Lindane) & 1.4 & $\mathrm{U}$ & 1.4 & 1.4 & $\mathrm{U}$ & 1.4 & 1.3 & $\mathrm{U}$ & 1.3 & 1.4 & $\mathrm{U}$ & 1.4 \\
\hline gamma-Chlordane & 1.8 & $\mathrm{~J}$ & 1.4 & 1.4 & $\mathrm{U}$ & 1.4 & 1.3 & $\mathrm{U}$ & 1.3 & 1.4 & $\mathrm{U}$ & 1.4 \\
\hline Heptachlor & 1.4 & $\mathrm{U}$ & 1.4 & 1.4 & $\mathrm{U}$ & 1.4 & 1.3 & $\mathrm{U}$ & 1.3 & 1.4 & $\mathrm{U}$ & 1.4 \\
\hline Heptachlor epoxide & 1.4 & $\mathrm{U}$ & 1.4 & 1.4 & $\mathrm{U}$ & 1.4 & 1.3 & $\mathrm{U}$ & 1.3 & 1.4 & $\mathrm{U}$ & 1.4 \\
\hline Methoxychlor & 1.4 & $\mathrm{U}$ & 1.4 & 1.4 & $\mathrm{U}$ & 1.4 & 1.3 & $\mathrm{U}$ & 1.3 & 1.4 & $\mathrm{U}$ & 1.4 \\
\hline Toxaphene & 14 & $\mathrm{U}$ & 14 & 14 & $\mathrm{U}$ & 14 & 13 & $\mathrm{U}$ & 13 & 14 & $\mathrm{U}$ & 14 \\
\hline \multicolumn{13}{|c|}{ Semivolatile Organic Analytes } \\
\hline 1,2,4-Trichlorobenzene & 350 & $\mathrm{U}$ & 350 & 340 & $\mathrm{U}$ & 340 & 340 & $\mathrm{U}$ & 340 & 350 & $\mathrm{U}$ & 350 \\
\hline 1,2-Dichlorobenzene & 350 & $\mathrm{U}$ & 350 & 340 & $\mathrm{U}$ & 340 & 340 & $\mathrm{U}$ & 340 & 350 & $\mathrm{U}$ & 350 \\
\hline 1,3-Dichlorobenzene & 350 & $\mathrm{U}$ & 350 & 340 & $\mathrm{U}$ & 340 & 340 & $\mathrm{U}$ & 340 & 350 & $\mathrm{U}$ & 350 \\
\hline 1,4-Dichlorobenzene & 350 & $\mathrm{U}$ & 350 & 340 & $\mathrm{U}$ & 340 & 340 & $\mathrm{U}$ & 340 & 350 & $\mathrm{U}$ & 350 \\
\hline 2,4,5-Trichlorophenol & 880 & $\mathrm{U}$ & 880 & 850 & $\mathrm{U}$ & 850 & 840 & $\mathrm{U}$ & 840 & 880 & $\mathrm{U}$ & 880 \\
\hline 2,4,6-Trichlorophenol & 350 & $\mathrm{U}$ & 350 & 340 & $\mathrm{U}$ & 340 & 340 & $\mathrm{U}$ & 340 & 350 & $\mathrm{U}$ & 350 \\
\hline 2,4-Dichlorophenol & 350 & $\mathrm{U}$ & 350 & 340 & $\mathrm{U}$ & 340 & 340 & $\mathrm{U}$ & 340 & 350 & $\mathrm{U}$ & 350 \\
\hline 2,4-Dimethylphenol & 350 & $U$ & 350 & 340 & $\mathrm{U}$ & 340 & 340 & $\mathrm{U}$ & 340 & 350 & $\mathrm{U}$ & 350 \\
\hline 2,4-Dinitrophenol & 880 & $\mathrm{U}$ & 880 & 850 & $\mathrm{U}$ & 850 & 840 & $\mathrm{U}$ & 840 & 880 & $\mathrm{U}$ & 880 \\
\hline 2,4-Dinitrotoluene & 350 & $\mathrm{U}$ & 350 & 340 & $\mathrm{U}$ & 340 & 340 & $\mathrm{U}$ & 340 & 350 & $\mathrm{U}$ & 350 \\
\hline 2,6-Dinitrotoluene & 350 & $\mathrm{U}$ & 350 & 340 & $\mathrm{U}$ & 340 & 340 & $\mathrm{U}$ & 340 & 350 & $\mathrm{U}$ & 350 \\
\hline 2-Chloronaphthalene & 350 & $\mathrm{U}$ & 350 & 340 & $\mathrm{U}$ & 340 & 340 & $\mathrm{U}$ & 340 & 350 & $\mathrm{U}$ & 350 \\
\hline 2-Chlorophenol & 350 & $\mathrm{U}$ & 350 & 340 & $\mathrm{U}$ & 340 & 340 & $\mathrm{U}$ & 340 & 350 & $\mathrm{U}$ & 350 \\
\hline 2-Methylnaphthalene & 350 & $\mathrm{U}$ & 350 & 340 & $\mathrm{U}$ & 340 & 340 & $\mathrm{U}$ & 340 & 350 & $\mathrm{U}$ & 350 \\
\hline 2-Methylphenol (cresol, o-) & 350 & $\mathrm{U}$ & 350 & 340 & $\mathrm{U}$ & 340 & 340 & $\mathrm{U}$ & 340 & 350 & $\mathrm{U}$ & 350 \\
\hline 2-Nitroaniline & 880 & $U$ & 880 & 850 & $\mathrm{U}$ & 850 & 840 & $\mathrm{U}$ & 840 & 880 & $\mathrm{U}$ & 880 \\
\hline 2-Nitrophenol & 350 & $\mathrm{U}$ & 350 & 340 & $\mathrm{U}$ & 340 & 340 & $U$ & 340 & 350 & $U$ & 350 \\
\hline & & & & & & Attachment & & 1 & & Sheet No. & & 3 of 18 \\
\hline & & & & & & Originator & $\mathrm{H.N}$ & Sul & vay & Date & & 4/03/08 \\
\hline & & & & & & Checked & & D. $\mathrm{H}$ & & Datc & & 4/03/08 \\
\hline & & & & & & Calc. No. & 0100 & $\mathrm{CA}$ & 0350 & Rev. No. & & 0 \\
\hline
\end{tabular}


Attachment 1. 120-F-1 Verification Sampling Results.

\begin{tabular}{|c|c|c|c|c|c|c|c|c|c|c|c|c|}
\hline \multirow[t]{2}{*}{ Constituent } & \multicolumn{3}{|c|}{$\begin{array}{c}\text { J16DTs } \\
\text { Sample Location NW-2 } \\
\text { Sample Date } 3 / 18 / 07\end{array}$} & \multicolumn{3}{|c|}{$\begin{array}{c}\text { J16DT9 } \\
\text { Sample Location NW-3 } \\
\text { Sample Date 3/18/07 }\end{array}$} & \multicolumn{3}{|c|}{$\begin{array}{c}\text { J16DV0 } \\
\text { Sample Location NW-4 } \\
\text { Sample Date } 3 / 18 / 07\end{array}$} & \multicolumn{3}{|c|}{$\begin{array}{c}\text { J16DV1 } \\
\text { Sample Location NW-5 } \\
\text { Sample Date 3/18/07 }\end{array}$} \\
\hline & $\mu \mathrm{g} / \mathrm{kg}$ & $Q$ & PQL & $\mu \mathrm{g} / \mathrm{kg}$ & $Q$ & PQL & $\mu \mathrm{g} / \mathrm{kg}$ & $\mathrm{Q}$ & PQL & $\mu \mathrm{g} / \mathrm{kg}$ & Q & PQL \\
\hline \multicolumn{13}{|c|}{ Semivolatile Organic Analytes (continued) } \\
\hline 3,3'-Dichlorobenzidine & 350 & $\mathrm{U}$ & 350 & 340 & $\mathrm{U}$ & 340 & 340 & $\mathrm{U}$ & 340 & 350 & $\mathrm{U}$ & 350 \\
\hline 4-Methylphenol (p-cresol) & 350 & $\mathrm{U}$ & 350 & 340 & $\mathrm{U}$ & 340 & 340 & $\mathrm{U}$ & 340 & 350 & $\mathrm{U}$ & 350 \\
\hline 3-Nitroaniline & 880 & $\mathrm{U}$ & 880 & 850 & $\mathrm{U}$ & 850 & 840 & $\mathrm{U}$ & 840 & 880 & $\mathrm{U}$ & 880 \\
\hline 4,6-Dinitro-2-methylphenol & 880 & $\mathrm{U}$ & 880 & 850 & $\mathrm{U}$ & 850 & 840 & $\mathrm{U}$ & 840 & 880 & $\mathrm{U}$ & 880 \\
\hline 4-Bromophenyl-phenylether & 350 & $\mathrm{U}$ & 350 & 340 & $\mathrm{U}$ & 340 & 340 & $\mathrm{U}$ & 340 & 350 & $\mathrm{U}$ & 350 \\
\hline 4-Chloro-3-methylphenol & 350 & $\mathrm{U}$ & 350 & 340 & $\mathrm{U}$ & 340 & 340 & $\mathrm{U}$ & 340 & 350 & $\mathrm{U}$ & 350 \\
\hline 4-Chloroaniline & 350 & $\mathrm{U}$ & 350 & 340 & $\mathrm{U}$ & 340 & 340 & $\mathrm{U}$ & 340 & 350 & $\mathrm{U}$ & 350 \\
\hline 4-Chlorophenyl-phenylether & 350 & $\mathrm{U}$ & 350 & 340 & $\mathrm{U}$ & 340 & 340 & $\mathrm{U}$ & 340 & 350 & $\mathrm{U}$ & 350 \\
\hline 4-Nitroaniline & 880 & $\mathrm{U}$ & 880 & 850 & $\mathrm{U}$ & 850 & 840 & $\mathrm{U}$ & 840 & 880 & $\mathrm{U}$ & 880 \\
\hline 4-Nitrophenol & 880 & $\mathrm{U}$ & 880 & 850 & $\mathrm{U}$ & 850 & 840 & $\mathrm{U}$ & 840 & 880 & $\mathrm{U}$ & 880 \\
\hline Acenaphthene & 350 & $\mathrm{U}$ & 350 & 340 & $\mathrm{U}$ & 340 & 340 & $\mathrm{U}$ & 340 & 350 & $\mathrm{U}$ & 350 \\
\hline Acenaphthylene & 350 & $\mathrm{U}$ & 350 & 340 & $\mathrm{U}$ & 340 & 340 & $\mathrm{U}$ & 340 & 350 & $\mathrm{U}$ & 350 \\
\hline Anthracene & 350 & $\mathrm{U}$ & 350 & 340 & $\mathrm{U}$ & 340 & 340 & $\mathrm{U}$ & 340 & 350 & $\mathrm{U}$ & 350 \\
\hline Benzo(a)anthracene & 350 & $\mathrm{U}$ & 350 & 340 & $\mathrm{U}$ & 340 & 340 & $\mathrm{U}$ & 340 & 350 & $\mathrm{U}$ & 350 \\
\hline Benzo(a)pyrene & 350 & $\mathrm{U}$ & 350 & 340 & $\mathrm{U}$ & 340 & 340 & $\mathrm{U}$ & 340 & 350 & $\mathrm{U}$ & 350 \\
\hline Benzo(b)fluoranthene & 350 & $\mathrm{U}$ & 350 & 340 & $\mathrm{U}$ & 340 & 340 & $\mathrm{U}$ & 340 & 350 & $\mathrm{U}$ & 350 \\
\hline Benzo $(\mathrm{g}, \mathrm{h}, \mathrm{i})$ perylene & 350 & $\mathrm{U}$ & 350 & 340 & $\mathrm{U}$ & 340 & 340 & $\mathrm{U}$ & 340 & 350 & $\mathrm{U}$ & 350 \\
\hline Benzo(k)fluoranthene & 350 & $\mathrm{U}$ & 350 & 340 & $\mathrm{U}$ & 340 & 340 & $\mathrm{U}$ & 340 & 350 & $\mathrm{U}$ & 350 \\
\hline Bis(2-chloro-1-methylethyl)ether & 350 & $\mathrm{U}$ & 350 & 340 & $\mathrm{U}$ & 340 & 340 & $\mathrm{U}$ & 340 & 350 & $\mathrm{U}$ & 350 \\
\hline Bis(2-chloroethoxy)methane & 350 & $\mathrm{U}$ & 350 & 340 & $\mathrm{U}$ & 340 & 340 & $\mathrm{U}$ & 340 & 350 & $\mathrm{U}$ & 350 \\
\hline Bis(2-chloroethyl) ether & 350 & $\mathrm{U}$ & 350 & 340 & $\mathrm{U}$ & 340 & 340 & $\mathrm{U}$ & 340 & 350 & $\mathrm{U}$ & 350 \\
\hline Bis(2-ethylhexyl) phthalate & 100 & $\mathrm{JB}$ & 350 & 84 & $\mathrm{JB}$ & 340 & 72 & $\mathrm{JB}$ & 340 & 31 & $\mathrm{JB}$ & 350 \\
\hline Butylbenzylphthalate & 350 & $\mathrm{U}$ & 350 & 340 & $\mathrm{U}$ & 340 & 340 & $\mathrm{U}$ & 340 & 350 & $\mathrm{U}$ & 350 \\
\hline Carbazole & 350 & $\mathrm{U}$ & 350 & 340 & $\mathrm{U}$ & 340 & 340 & $\mathrm{U}$ & 340 & 350 & $\mathrm{U}$ & 350 \\
\hline Chrysene & 350 & $\mathrm{U}$ & 350 & 340 & $\mathrm{U}$ & 340 & 340 & $\mathrm{U}$ & 340 & 350 & $\bar{U}$ & 350 \\
\hline Dibenz(a,h)anthracene & 350 & $\mathrm{U}$ & 350 & 340 & $\mathrm{U}$ & 340 & 340 & $\mathrm{U}$ & 340 & 350 & $\mathrm{U}$ & 350 \\
\hline Dibenzofuran & 350 & $\mathrm{U}$ & 350 & 340 & U & 340 & 340 & $\mathrm{U}$ & 340 & 350 & $\mathrm{U}$ & 350 \\
\hline Diethylphthalate & 350 & $\mathrm{U}$ & 350 & 340 & $\mathrm{U}$ & 340 & 340 & $\mathrm{U}$ & 340 & 350 & $\mathrm{U}$ & 350 \\
\hline Dimethylphthalate & 350 & $\mathrm{U}$ & 350 & 340 & $\mathrm{U}$ & 340 & 340 & $\mathrm{U}$ & 340 & 350 & $\mathrm{U}$ & 350 \\
\hline Di-n-butylphthalate & 19 & $\mathrm{~J}$ & 350 & 27 & $\mathrm{~J}$ & 340 & 25 & $\mathrm{~J}$ & 340 & 350 & $\mathrm{U}$ & 350 \\
\hline Di-n-octylphthalate & 350 & $\mathrm{U}$ & 350 & 340 & $\mathrm{U}$ & 340 & 340 & $\mathrm{U}$ & 340 & 350 & $\mathrm{U}$ & 350 \\
\hline Fluoranthene & 350 & $\mathrm{U}$ & 350 & 340 & $\mathrm{U}$ & 340 & 340 & $\mathrm{U}$ & 340 & 350 & $\mathrm{U}$ & 350 \\
\hline Fluorene & 350 & $\mathrm{U}$ & 350 & 340 & $U$ & 340 & 340 & $\mathrm{U}$ & 340 & 350 & $\mathrm{U}$ & 350 \\
\hline Hexachlorobenzene & 350 & $\mathrm{U}$ & 350 & 340 & $\mathrm{U}$ & 340 & 340 & $\mathrm{U}$ & 340 & 350 & $\mathrm{U}$ & 350 \\
\hline Hexachlorobutadiene & 350 & $\mathrm{U}$ & 350 & 340 & $\mathrm{U}$ & 340 & 340 & $\mathrm{U}$ & 340 & 350 & $U$ & 350 \\
\hline Hexachlorocyclopentadiene & 350 & $\mathrm{U}$ & 350 & 340 & $\mathrm{U}$ & 340 & 340 & $\mathrm{U}$ & 340 & 350 & $\mathrm{U}$ & 350 \\
\hline Hexachloroethane & 350 & $\mathrm{U}$ & 350 & 340 & $\mathrm{U}$ & 340 & 340 & $\mathrm{U}$ & 340 & 350 & $\mathrm{U}$ & 350 \\
\hline Indeno(1,2,3-cd)pyrene & 350 & $\mathrm{U}$ & 350 & 340 & $\mathrm{U}$ & 340 & 340 & $\mathrm{U}$ & 340 & 350 & $\mathrm{U}$ & 350 \\
\hline Isophorone & 350 & $\mathrm{U}$ & 350 & 340 & $\mathrm{U}$ & 340 & 340 & $\mathrm{U}$ & 340 & 350 & $\mathrm{U}$ & 350 \\
\hline Naphthalene & 350 & $\mathrm{U}$ & 350 & 340 & $\mathrm{U}$ & 340 & 340 & $\mathrm{U}$ & 340 & 350 & $U$ & 350 \\
\hline Nitrobenzene & 350 & $\mathrm{U}$ & 350 & 340 & $\mathrm{U}$ & 340 & 340 & $\mathrm{U}$ & 340 & 350 & $\mathrm{U}$ & 350 \\
\hline N-Nitroso-di-n-dipropylamine & 350 & $\mathrm{U}$ & 350 & 340 & $\mathrm{U}$ & 340 & 340 & $\mathrm{U}$ & 340 & 350 & $U$ & 350 \\
\hline N-Nitrosodiphenylamine & 350 & $\mathrm{U}$ & 350 & 340 & $\mathrm{U}$ & 340 & 340 & $\mathrm{U}$ & 340 & 350 & $\mathrm{U}$ & 350 \\
\hline Pentachlorophenol & 880 & $\mathrm{U}$ & 880 & 850 & $\mathrm{U}$ & 850 & 840 & $\mathrm{U}$ & 840 & 880 & $\mathrm{U}$ & 880 \\
\hline Phenanthrene & 350 & $\mathrm{U}$ & 350 & 340 & $\mathrm{U}$ & 340 & 340 & $\mathrm{U}$ & 340 & 350 & $\mathrm{U}$ & 350 \\
\hline Phenol & 350 & $\mathrm{U}$ & 350 & 340 & $\mathrm{U}$ & 340 & 340 & $\mathrm{U}$ & 340 & 350 & $\mathrm{U}$ & 350 \\
\hline Pyrene & 350 & $\mathrm{U}$ & 350 & 340 & $\mathrm{U}$ & 340 & 340 & $\mathrm{U}$ & 340 & 350 & $\mathrm{U}$ & 350 \\
\hline & & & & & & Attachment & & 1 & & Sheet No. & & 4 of 18 \\
\hline & & & & & & Originator & & Sul & way & Date & & $4 / 03 / 08$ \\
\hline & & & & & & Checked & & D. H & & Date & & $4 / 03 / 08$ \\
\hline & & & & & & Calc. No. & 010 & $\mathrm{CA}$ & 10350 & Rev. No. & & 0 \\
\hline
\end{tabular}


Attachment 1. 120-F-1 Verification Sampling Results.

\begin{tabular}{|c|c|c|c|c|c|c|c|c|c|c|c|c|}
\hline \multirow[t]{2}{*}{ Constituent } & \multicolumn{3}{|c|}{$\begin{array}{c}\text { J16DV2 } \\
\text { Sample Location NW-6 } \\
\text { Sample Date 3/18/07 }\end{array}$} & \multicolumn{3}{|c|}{$\begin{array}{c}\text { J16DV3 } \\
\text { Sample Location NW-7 } \\
\text { Sample Date } 3 / 18 / 07\end{array}$} & \multicolumn{3}{|c|}{$\begin{array}{c}\text { J16DV4 } \\
\text { Sample Location NW-8 } \\
\text { Sample Date 3/18/07 }\end{array}$} & \multicolumn{3}{|c|}{$\begin{array}{c}\text { J16DV5 } \\
\text { Sample Location NW-9 } \\
\text { Sample Date 3/18/07 }\end{array}$} \\
\hline & $\mu \mathrm{g} / \mathrm{kg}$ & $Q$ & PQL & $\mu \mathrm{g} / \mathrm{kg}$ & $Q$ & PQL & $\mu \mathrm{g} / \mathrm{kg}$ & $Q$ & PQL & $\mu \mathrm{g} / \mathrm{kg}$ & $\mathrm{Q}$ & PQL \\
\hline \multicolumn{13}{|c|}{ Polychlorinated Biphenyls } \\
\hline Aroclor-1016 & 14 & $\mathrm{U}$ & 14 & 14 & U & 14 & 14 & U & 14 & 14 & U & 14 \\
\hline Aroclor-1221 & 14 & $\mathrm{U}$ & 14 & 14 & $\mathrm{U}$ & 14 & 14 & $\mathrm{U}$ & 14 & 14 & $\mathrm{U}$ & 14 \\
\hline Aroclor-1232 & 14 & $\mathrm{U}$ & 14 & 14 & $\mathrm{U}$ & 14 & 14 & $\mathrm{U}$ & 14 & 14 & $\mathrm{U}$ & 14 \\
\hline Aroclor- 1242 & 14 & $\mathrm{U}$ & 14 & 14 & $\mathrm{U}$ & 14 & 14 & $\mathrm{U}$ & 14 & 14 & $\mathrm{U}$ & 14 \\
\hline Aroclor- 1248 & 14 & $\mathrm{U}$ & 14 & 14 & $\mathrm{U}$ & 14 & 14 & $\mathrm{U}$ & 14 & 14 & \begin{tabular}{|l|}
$U$ \\
\end{tabular} & 14 \\
\hline Aroclor- 1254 & 14 & U & 14 & 14 & $\mathrm{U}$ & 14 & 14 & $\mathrm{U}$ & 14 & 14 & $\mathrm{U}$ & 14 \\
\hline Aroclor- 1260 & 14 & $\mathrm{U}$ & 14 & 14 & $\mathrm{U}$ & 14 & 14 & $\mathrm{U}$ & 14 & 14 & $\mathrm{U}$ & 14 \\
\hline \multicolumn{13}{|c|}{ Pesticides } \\
\hline Aldrin & 1.4 & $\mathrm{U}$ & 1.4 & 1.4 & U & 1.4 & 1.4 & $\mathrm{U}$ & 1.4 & 1.4 & U & 1.4 \\
\hline Alpha-BHC & 1.4 & U & 1.4 & 1.4 & $\mathrm{U}$ & 1.4 & 1.4 & $\mathrm{U}$ & 1.4 & 1.4 & \begin{tabular}{|l|}
$\mathrm{U}$ \\
\end{tabular} & 1.4 \\
\hline Alpha-Chlordane & 1.4 & $\mathrm{U}$ & 1.4 & 1.4 & $\mathrm{U}$ & 1.4 & 2.1 & $\mathrm{~J}$ & 1.4 & 1.4 & $\mathrm{U}$ & 1.4 \\
\hline Beta-BHC & 1.4 & $\mathrm{U}$ & 1.4 & 1.4 & $\mathrm{U}$ & 1.4 & 1.4 & $\mathrm{U}$ & 1.4 & 1.4 & \begin{tabular}{|l|l}
$U$ \\
\end{tabular} & 1.4 \\
\hline Delta-BHC & 1.4 & $\mathrm{U}$ & 1.4 & 1.4 & $\mathrm{U}$ & 1.4 & 1.4 & $\mathrm{U}$ & 1.4 & 1.4 & $\mathrm{U}$ & 1.4 \\
\hline Dichlorodiphenyldichloroethane & 1.4 & $\mathrm{U}$ & 1.4 & 1.4 & $\mathrm{U}$ & 1.4 & 1.4 & $\mathrm{U}$ & 1.4 & 1.4 & $\mathrm{U}$ & 1.4 \\
\hline Dichlorodiphenyldichloroetlyylene & 1.4 & $\mathrm{U}$ & 1.4 & 1.4 & $\mathrm{U}$ & 1.4 & 1.4 & $\mathrm{U}$ & 1.4 & 1.4 & $\mathrm{U}$ & 1.4 \\
\hline Dichlorodiphenyltrichloroethane & 1.4 & $\mathrm{U}$ & 1.4 & 1.4 & $\mathrm{U}$ & 1.4 & 1.4 & $\mathrm{U}$ & 1.4 & 1.4 & $\mathrm{U}$ & 1.4 \\
\hline Dieldrin & 1.4 & $\mathrm{U}$ & 1.4 & 1.4 & $\mathrm{U}$ & 1.4 & 1.4 & $\mathrm{U}$ & 1.4 & 1.4 & U & 1.4 \\
\hline Endosulfan I & 1.4 & $\mathrm{U}$ & 1.4 & 1.4 & $\mathrm{U}$ & 1.4 & 1.4 & $\mathrm{U}$ & 1.4 & 1.4 & $\mathrm{U}$ & 1.4 \\
\hline Endosulfan II & 1.4 & $\mathrm{U}$ & 1.4 & 1.4 & U & 1.4 & 1.4 & $\mathrm{U}$ & 1.4 & 1.4 & U & 1.4 \\
\hline Endosulfan sulfate & 1.4 & $\mathrm{U}$ & 1.4 & 1.4 & $\mathrm{U}$ & 1.4 & 1.4 & $\mathrm{U}$ & 1.4 & 1.4 & \begin{tabular}{|l|}
$U$ \\
\end{tabular} & 1.4 \\
\hline Endrin & 1.4 & U & 1.4 & 1.4 & U & 1.4 & 1.4 & $\mathrm{U}$ & 1.4 & 1.4 & $\mathrm{U}$ & 1.4 \\
\hline Endrin aldehyde & 1.4 & U & 1.4 & 1.4 & U & 1.4 & 1.4 & $\mathrm{U}$ & 1.4 & 1.4 & $\mathrm{U}$ & 1.4 \\
\hline Endrin ketone & 1.4 & $\mathrm{U}$ & 1.4 & 1.4 & $U$ & 1.4 & 1.4 & $\mathrm{U}$ & 1.4 & 1.4 & $\mathrm{U}$ & 1.4 \\
\hline Gamma-BHC (Lindane) & 1.4 & $\mathrm{U}$ & 1.4 & 1.4 & U & 1.4 & 1.4 & $\mathrm{U}$ & 1.4 & 1.4 & $\mathrm{U}$ & 1.4 \\
\hline gamma-Chlordane & 1.4 & $\mathrm{U}$ & 1.4 & 1.4 & U & 1.4 & 2.2 & $\mathrm{~J}$ & 1.4 & 1.4 & $\mathrm{U}$ & 1.4 \\
\hline Heptachlor & 1.4 & U & 1.4 & 1.4 & U & 1.4 & 1.4 & $\mathrm{U}$ & 1.4 & 1.4 & $\mathrm{U}$ & 1.4 \\
\hline Heptachlor epoxide & 1.4 & U & 1.4 & 1.4 & $\mathrm{U}$ & 1.4 & 1.4 & $\mathrm{U}$ & 1.4 & 1.4 & $\mathrm{U}$ & 1.4 \\
\hline Methoxychlor & 1.4 & $\mathrm{U}$ & 1.4 & 1.4 & $U$ & 1.4 & 1.4 & $\mathrm{U}$ & 1.4 & 1.4 & $\mathrm{U}$ & 1.4 \\
\hline Toxaphene & 14 & $\mathrm{U}$ & 14 & 14 & $\mathrm{U}$ & 14 & 14 & $\mathrm{U}$ & 14 & 14 & $\mathrm{U}$ & 14 \\
\hline \multicolumn{13}{|c|}{ Semivolatile Organic Analytes } \\
\hline 1,2,4-Trichlorobenzene & 340 & $\mathrm{U}$ & 340 & 340 & $\mathrm{U}$ & 340 & 360 & $\mathrm{U}$ & 360 & 340 & $\mathrm{U}$ & 340 \\
\hline 1,2-Dichlorobenzene & 340 & $\mathrm{U}$ & 340 & 340 & $\mathrm{U}$ & 340 & 360 & $\mathrm{U}$ & 360 & 340 & $\mathrm{U}$ & 340 \\
\hline 1,3-Dichlorobenzene & 340 & $\mathrm{U}$ & 340 & 340 & $\mathrm{U}$ & 340 & 360 & $\mathrm{U}$ & 360 & 340 & $\mathrm{U}$ & 340 \\
\hline 1,4-Dichlorobenzene & 340 & $\mathrm{U}$ & 340 & 340 & $\mathrm{U}$ & 340 & 360 & $\mathrm{U}$ & 360 & 340 & U & 340 \\
\hline 2,4,5-Trichlorophenol & 860 & $\mathrm{U}$ & 860 & 840 & $\mathrm{U}$ & 840 & 900 & $\mathrm{U}$ & 900 & 860 & \begin{tabular}{|l|}
$\mathrm{U}$ \\
\end{tabular} & 860 \\
\hline $2,4,6$-Trichlorophenol & 340 & U & 340 & 340 & $\mathrm{U}$ & 340 & 360 & $\mathrm{U}$ & 360 & 340 & $\mathrm{U}$ & 340 \\
\hline 2,4-Dichlorophenol & 340 & $\mathrm{U}$ & 340 & 340 & U & 340 & 360 & $\mathrm{U}$ & 360 & 340 & U & 340 \\
\hline 2,4-Dimethylphenol & 340 & $\mathrm{U}$ & 340 & 340 & $\mathrm{U}$ & 340 & 360 & $\mathrm{U}$ & 360 & 340 & U & 340 \\
\hline 2,4-Dinitrophenol & 860 & $\mathrm{U}$ & 860 & 840 & $\mathrm{U}$ & 840 & 900 & $\mathrm{U}$ & 900 & 860 & U & 860 \\
\hline 2,4-Dinitrotoluene & 340 & $\mathrm{U}$ & 340 & 340 & $\mathrm{U}$ & 340 & 360 & U & 360 & 340 & $\mathrm{U}$ & 340 \\
\hline 2,6-Dinitrotoluene & 340 & $\mathrm{U}$ & 340 & 340 & U & 340 & 360 & $\mathrm{U}$ & 360 & 340 & U & 340 \\
\hline 2-Chloronaphthalene & 340 & $\mathrm{U}$ & 340 & 340 & $\mathrm{U}$ & 340 & 360 & $\mathrm{U}$ & 360 & 340 & $\mathrm{U}$ & 340 \\
\hline 2-Chlorophenol & 340 & U & 340 & 340 & 0 & 340 & 360 & $\mathrm{U}$ & 360 & 340 & $\mathrm{U}$ & 340 \\
\hline 2-Methylnaphthalene & 340 & $\mathrm{U}$ & 340 & 340 & $\mathrm{U}$ & 340 & 360 & $\mathrm{U}$ & 360 & 340 & U & 340 \\
\hline 2-Methylphenol (cresol, o-) & 340 & $\mathrm{U}$ & 340 & 340 & $\mathrm{U}$ & 340 & 360 & U & 360 & 340 & \begin{tabular}{|l|}
$U$ \\
\end{tabular} & 340 \\
\hline 2-Nitroaniline & 860 & $\mathrm{U}$ & 860 & 840 & $\mathrm{U}$ & 840 & 900 & U & 900 & 860 & U & 860 \\
\hline 2-Nitrophenol & 340 & U & 340 & 340 & $\mathrm{U}$ & 340 & 360 & $\mathrm{U}$ & 360 & 340 & $\mathrm{U}$ & 340 \\
\hline & & & & & & Altachment & & 1 & & Sheet No. & & 15 or 18 \\
\hline & & & & & & Originator & $\mathrm{H}$. & Sul & vay & Date & & $04 / 03 / 08$ \\
\hline & & & & & & Checked & & . 1. & & Date & & $04 / 03 / 08$ \\
\hline & & & & & & Calc. No. & 0100 & $\mathrm{CA}$ & 0350 & Rev. No. & & 0 \\
\hline
\end{tabular}


Attachment 1. 120-F-1 Verification Sampling Results.

\begin{tabular}{|c|c|c|c|c|c|c|c|c|c|c|c|c|}
\hline \multirow[t]{2}{*}{ Constituent } & \multicolumn{3}{|c|}{$\begin{array}{c}\text { J16DV2 } \\
\text { Sample Location NW-6 } \\
\text { Sample Date 3/18/07 }\end{array}$} & \multicolumn{3}{|c|}{$\begin{array}{c}\text { J16DV3 } \\
\text { Sample Location NW-7 } \\
\text { Sample Date } 3 / 18 / 07\end{array}$} & \multicolumn{3}{|c|}{$\begin{array}{c}\text { J16DV4 } \\
\text { Sample Location NW-8 } \\
\text { Sample Date } 3 / 18 / 07\end{array}$} & \multicolumn{3}{|c|}{$\begin{array}{c}\text { J16DV5 } \\
\text { Sample Location NW-9 } \\
\text { Sample Date 3/18/07 }\end{array}$} \\
\hline & $\mu \mathrm{g} / \mathrm{kgg}$ & $Q$ & PQL & $\mu \mathrm{g} / \mathrm{kg}$ & $Q$ & PQL & $\mu \mathrm{g} / \mathrm{kg}$ & $Q$ & PQL & $\mu \mathrm{g} / \mathrm{kg}$ & $Q$ & PQL \\
\hline \multicolumn{13}{|c|}{ Semivolatile Organic Analytes (continued) } \\
\hline 3,3'-Dichlorobenzidine & 340 & $\mathrm{U}$ & 340 & 340 & $\mathrm{U}$ & 340 & 360 & $\mathrm{U}$ & 360 & 340 & $\mathrm{U}$ & 340 \\
\hline 4-Methylphenol (p-cresol) & 340 & U & 340 & 340 & $U$ & 340 & 360 & $\mathrm{U}$ & 360 & 340 & $\mathrm{U}$ & 340 \\
\hline 3-Nitroaniline & 860 & U & 860 & 840 & $\mathrm{U}$ & 840 & 900 & $\mathrm{U}$ & 900 & 860 & $\mathrm{U}$ & 860 \\
\hline 4,6-Dinitro-2-methylphenol & 860 & U & 860 & 840 & $\mathrm{U}$ & 840 & 900 & $\mathrm{U}$ & 900 & 860 & $\mathrm{U}$ & 860 \\
\hline 4-Bromophenyl-phenylether & 340 & $\mathrm{U}$ & 340 & 340 & U & 340 & 360 & $\mathrm{U}$ & 360 & 340 & $\mathrm{U}$ & 340 \\
\hline 4-Chloro-3-methylphenol & 340 & $\mathrm{U}$ & 340 & 340 & U & 340 & 360 & $\mathrm{U}$ & 360 & 340 & $\mathrm{U}$ & 340 \\
\hline 4-Chloroaniline & 340 & U & 340 & 340 & $\mathrm{U}$ & 340 & 360 & $\mathrm{U}$ & 360 & 340 & $\mathrm{U}$ & 340 \\
\hline 4-Chlorophenyl-phenylether & 340 & U & 340 & 340 & U & 340 & 360 & $\mathrm{U}$ & 360 & 340 & $\mathrm{U}$ & 340 \\
\hline 4-Nitroaniline & 860 & U & 860 & 840 & $\mathrm{U}$ & 840 & 900 & $\mathrm{U}$ & 900 & 860 & $\mathrm{U}$ & 860 \\
\hline 4-Nitrophenol & 860 & U & 860 & 840 & $\mathrm{U}$ & 840 & 900 & $\mathrm{U}$ & 900 & 860 & U & 860 \\
\hline Acenaphthene & 340 & U & 340 & 340 & $\mathrm{U}$ & 340 & 360 & $\mathrm{U}$ & 360 & 340 & $\mathrm{U}$ & 340 \\
\hline Acenaphthylene & 340 & U & 340 & 340 & $\mathrm{U}$ & 340 & 360 & $\mathrm{U}$ & 360 & 340 & \begin{tabular}{|l|}
$\mathrm{U}$ \\
\end{tabular} & 340 \\
\hline \begin{tabular}{|l} 
Anthracene \\
\end{tabular} & 340 & $\mathrm{U}$ & 340 & 340 & $\mathrm{U}$ & 340 & 360 & $\mathrm{U}$ & 360 & 340 & U & 340 \\
\hline Benzo(a)anthracene & 340 & $\mathrm{U}$ & 340 & 340 & $\mathrm{U}$ & 340 & 360 & $\mathrm{U}$ & 360 & 340 & \begin{tabular}{|l|}
$U$ \\
\end{tabular} & 340 \\
\hline Benzo(a)pyrene & 340 & $\mathrm{U}$ & 340 & 340 & $\mathrm{U}$ & 340 & 360 & $\mathrm{U}$ & 360 & 340 & $\mathrm{U}$ & 340 \\
\hline Benzo(b)fluoranthene & 340 & $\mathrm{U}$ & 340 & 340 & $\mathrm{U}$ & 340 & 360 & $\mathrm{U}$ & 360 & 340 & $\mathrm{U}$ & 340 \\
\hline Benzo(g,h,i)perylene & 340 & $\mathrm{U}$ & 340 & 340 & $\mathrm{U}$ & 340 & 360 & $\mathrm{U}$ & 360 & 340 & $\mathrm{U}$ & 340 \\
\hline Benzo(k)fluoranthene & 340 & $\mathrm{U}$ & 340 & 340 & $\mathrm{U}$ & 340 & 360 & $\mathrm{U}$ & 360 & 340 & $\mathrm{U}$ & 340 \\
\hline Bis(2-chloro-1-methylethyl)ether & 340 & U & 340 & 340 & $\mathrm{U}$ & 340 & 360 & $\mathrm{U}$ & 360 & 340 & $\mathrm{U}$ & 340 \\
\hline Bis(2-chloroethoxy)methane & 340 & U & 340 & 340 & $\mathrm{U}$ & 340 & 360 & $\mathrm{U}$ & 360 & 340 & \begin{tabular}{|l|l}
$U$ \\
\end{tabular} & 340 \\
\hline Bis(2-chloroethyl) ether & 340 & $\mathrm{U}$ & 340 & 340 & $\mathrm{U}$ & 340 & 360 & $\mathrm{U}$ & 360 & 340 & \begin{tabular}{|l|}
$\mathrm{U}$ \\
\end{tabular} & 340 \\
\hline Bis(2-ethylhexyl) phthalate & 23 & $J B$ & 340 & 340 & $\mathrm{U}$ & 340 & 64 & $\mathrm{JB}$ & 360 & 29 & $\mathrm{JB}$ & 340 \\
\hline Butylbenzylphthalate & 340 & $\mathrm{U}$ & 340 & 340 & $\mathrm{U}$ & 340 & 360 & $\mathrm{U}$ & 360 & 340 & U & 340 \\
\hline Carbazole & 340 & $\mathrm{U}$ & 340 & 340 & $\mathrm{U}$ & 340 & 360 & $\mathrm{U}$ & 360 & 340 & $\mathrm{U}$ & 340 \\
\hline Chrysene & 340 & U & 340 & 340 & $\mathrm{U}$ & 340 & 360 & $\mathrm{U}$ & 360 & 340 & $\mathrm{U}$ & 340 \\
\hline Dibenz $(\mathrm{a}, \mathrm{h})$ anthracene & 340 & $\mathrm{U}$ & 340 & 340 & $U$ & 340 & 360 & $\mathrm{U}$ & 360 & 340 & $\mathrm{U}$ & 340 \\
\hline \begin{tabular}{|l|} 
Dibenzofuran \\
\end{tabular} & 340 & U & 340 & 340 & $\mathrm{U}$ & 340 & 360 & $\mathrm{U}$ & 360 & 340 & $\mathrm{U}$ & 340 \\
\hline Diethylphthalate & 340 & $\mathrm{U}$ & 340 & 340 & \begin{tabular}{|l|}
$U$ \\
\end{tabular} & 340 & 360 & $\mathrm{U}$ & 360 & 340 & $\mathrm{U}$ & 340 \\
\hline Dimethylphthalate & 340 & $\mathrm{U}$ & 340 & 340 & $\mathrm{U}$ & 340 & 360 & $\mathrm{U}$ & 360 & 340 & $\mathrm{U}$ & 340 \\
\hline Di-n-butylphthalate & 340 & U & 340 & 340 & $\mathrm{U}$ & 340 & 360 & $\mathrm{U}$ & 360 & 340 & $\mathrm{U}$ & 340 \\
\hline Di-n-octylphthalate & 340 & $\mathrm{U}$ & 340 & 340 & $\mathrm{U}$ & 340 & 360 & $\mathrm{U}$ & 360 & 340 & $\mathrm{U}$ & 340 \\
\hline Fluoranthene & 340 & $\mathrm{U}$ & 340 & 340 & $\mathrm{U}$ & 340 & 360 & $\mathrm{U}$ & 360 & 340 & U & 340 \\
\hline \begin{tabular}{|l} 
Fluorene \\
\end{tabular} & 340 & $\mathrm{U}$ & 340 & 340 & $\mathrm{U}$ & 340 & 360 & $\mathrm{U}$ & 360 & 340 & $\mathrm{U}$ & 340 \\
\hline Hexachlorobenzene & 340 & U & 340 & 340 & $\mathrm{U}$ & 340 & 360 & $\mathrm{U}$ & 360 & 340 & $\mathrm{U}$ & 340 \\
\hline Hexachlorobutadiene & 340 & $\mathrm{U}$ & 340 & 340 & $\mathrm{U}$ & 340 & 360 & $\mathrm{U}$ & 360 & 340 & $\mathrm{U}$ & 340 \\
\hline Hexachlorocyclopentadiene & 340 & $\mathrm{U}$ & 340 & 340 & $\mathrm{U}$ & 340 & 360 & $\mathrm{U}$ & 360 & 340 & $\mathrm{U}$ & 340 \\
\hline Hexachloroethane & 340 & U & 340 & 340 & $\mathrm{U}$ & 340 & 360 & $\mathrm{U}$ & 360 & 340 & $\mathrm{U}$ & 340 \\
\hline Indeno( $1,2,3$-cd)pyrene & 340 & U & 340 & 340 & $\mathrm{U}$ & 340 & 360 & $\mathrm{U}$ & 360 & 340 & $\mathrm{U}$ & 340 \\
\hline Isophorone & 340 & $\mathrm{U}$ & 340 & 340 & $\mathrm{U}$ & 340 & 360 & $\mathrm{U}$ & 360 & 340 & $\mathrm{U}$ & 340 \\
\hline Naphthalene & 340 & $\mathrm{U}$ & 340 & 340 & $\mathrm{U}$ & 340 & 360 & $\mathrm{U}$ & 360 & 340 & $\mathrm{U}$ & 340 \\
\hline Nitrobenzene & 340 & $\mathrm{U}$ & 340 & 340 & $\mathrm{U}$ & 340 & 360 & $\mathrm{U}$ & 360 & 340 & $\mathrm{U}$ & 340 \\
\hline N-Nitroso-di-n-dipropylamine & 340 & $\mathrm{U}$ & 340 & 340 & $\mathrm{U}$ & 340 & 360 & $\mathrm{U}$ & 360 & 340 & $\mathrm{U}$ & 340 \\
\hline N-Nitrosodiphenylamine & 340 & $\mathrm{U}$ & 340 & 340 & $\mathrm{U}$ & 340 & 360 & $\mathrm{U}$ & 360 & 340 & $\mathrm{U}$ & 340 \\
\hline Pentachlorophenol & 860 & $\mathrm{U}$ & 860 & 840 & $\mathrm{U}$ & 840 & 900 & $\mathrm{U}$ & 900 & 860 & $\mathrm{U}$ & 860 \\
\hline Phenanthrene & 340 & $\mathrm{U}$ & 340 & 340 & $\mathrm{U}$ & 340 & 360 & $\mathrm{U}$ & 360 & 340 & $\mathrm{U}$ & 340 \\
\hline Phenol & 340 & $\mathrm{U}$ & 340 & 340 & \begin{tabular}{|l|}
$U$ \\
\end{tabular} & 340 & 360 & $\mathrm{U}$ & 360 & 340 & $\mathrm{U}$ & 340 \\
\hline Pyrene & 340 & $\mathrm{U}$ & 340 & 340 & $\mathrm{U}$ & 340 & 360 & $\mathrm{U}$ & 360 & 340 & $\mathrm{U}$ & 340 \\
\hline & & & & & & Attachment & & 1 & & Sheet No. & & 6 of 18 \\
\hline & & & & & & Originator & H. & Sull & & Date & & $4 / 03 / 08$ \\
\hline & & & & & & Checked & & D. $\mathrm{Ha}$ & & Date & & $4 / 03 / 08$ \\
\hline & & & & & & Calc. No. & 0100 & $\mathrm{CA}-$ & 350 & Rev. No. & & 0 \\
\hline
\end{tabular}


Attachment 1. 120-F-1 Verification Sampling Results.

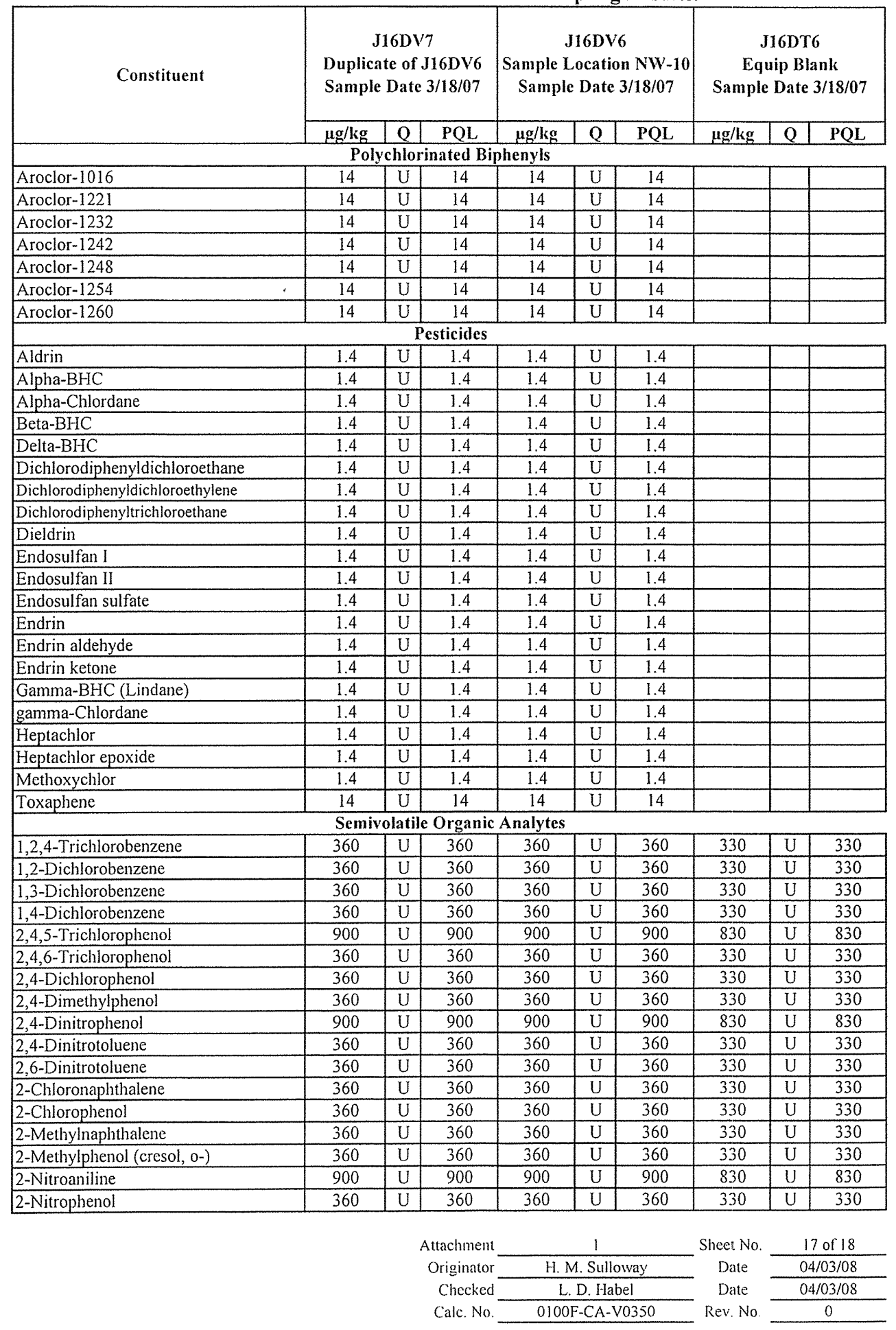


Attachment 1. 120-F-1 Verification Sampling Results.

\begin{tabular}{|c|c|c|c|c|c|c|c|c|c|}
\hline \multirow[t]{2}{*}{ Constituent } & \multicolumn{3}{|c|}{$\begin{array}{c}\text { J16DV7 } \\
\text { Duplicate of J16DV6 } \\
\text { Sample Date 3/18/07 }\end{array}$} & \multicolumn{3}{|c|}{$\begin{array}{c}\text { J16DV6 } \\
\text { Sample Location NW-10 } \\
\text { Sample Date 3/18/07 }\end{array}$} & \multicolumn{3}{|c|}{$\begin{array}{c}\text { J16D'T6 } \\
\text { Equip Blank } \\
\text { Sample Date } 3 / 18 / 07\end{array}$} \\
\hline & $\mu \mathrm{g} / \mathrm{kg}$ & $Q$ & PQL & $\mu \mathrm{g} / \mathrm{kg}$ & $Q$ & $\mathrm{PQL}$ & $\mu \mathrm{g} / \mathrm{kg}$ & $\mathrm{Q}$ & PQL \\
\hline \multicolumn{10}{|c|}{ Semivolatile Organic Analytes (continued) } \\
\hline 3,3'-Dichlorobenzidine & 360 & $\mathrm{U}$ & 360 & 360 & $\mathrm{U}$ & 360 & 330 & $\mathrm{U}$ & 330 \\
\hline 4-Methylphenol (p-cresol) & 360 & U & 360 & 360 & $\mathrm{U}$ & 360 & 330 & $\mathrm{U}$ & 330 \\
\hline 3-Nitroaniline & 900 & $\mathrm{U}$ & 900 & 900 & $\mathrm{U}$ & 900 & 830 & $\mathrm{U}$ & 830 \\
\hline 4,6-Dinitro-2-methylphenol & 900 & $\mathrm{U}$ & 900 & 900 & $\mathrm{U}$ & 900 & 830 & $\mathrm{U}$ & 830 \\
\hline 4-Bromophenyl-phenylether & 360 & $\mathrm{U}$ & 360 & 360 & $\mathrm{U}$ & 360 & 330 & $\mathrm{U}$ & 330 \\
\hline 4-Chloro-3-methylphenol & 360 & $\mathrm{U}$ & 360 & 360 & $\mathrm{U}$ & 360 & 330 & $\mathrm{U}$ & 330 \\
\hline 4-Chloroaniline & 360 & $\mathrm{U}$ & 360 & 360 & $\mathrm{U}$ & 360 & 330 & $\mathrm{U}$ & 330 \\
\hline 4-Chlorophenyl-phenylether & 360 & $\mathrm{U}$ & 360 & 360 & $\mathrm{U}$ & 360 & 330 & $\mathrm{U}$ & 330 \\
\hline 4-Nitroaniline & 900 & $\mathrm{U}$ & 900 & 900 & $\mathrm{U}$ & 900 & 830 & $\mathrm{U}$ & 830 \\
\hline 4-Nitrophenol & 900 & $\mathrm{U}$ & 900 & 900 & $\mathrm{U}$ & 900 & 830 & $\mathrm{U}$ & 830 \\
\hline Acenaphthene & 360 & $\mathrm{U}$ & 360 & 360 & $\mathrm{U}$ & 360 & 330 & $\mathrm{U}$ & 330 \\
\hline Acenaphthylene & 360 & $\mathrm{U}$ & 360 & 360 & $\mathrm{U}$ & 360 & 330 & $\mathrm{U}$ & 330 \\
\hline Anthracene & 360 & $\mathrm{U}$ & 360 & 360 & $\mathrm{U}$ & 360 & 330 & $\mathrm{U}$ & 330 \\
\hline Benzo(a)anthracene & 360 & $\mathrm{U}$ & 360 & 360 & $\mathrm{U}$ & 360 & 330 & $\mathrm{U}$ & 330 \\
\hline Benzo(a)pyrene & 360 & $\mathrm{U}$ & 360 & 360 & $\mathrm{U}$ & 360 & 330 & $\mathrm{U}$ & 330 \\
\hline Benzo(b)fluoranthene & 360 & $\mathrm{U}$ & 360 & 360 & $\mathrm{U}$ & 360 & 330 & $\mathrm{U}$ & 330 \\
\hline Benzo $(\mathrm{g}, \mathrm{h}, \mathrm{i})$ perylene & 360 & $\mathrm{U}$ & 360 & 360 & $\mathrm{U}$ & 360 & 330 & $\mathrm{U}$ & 330 \\
\hline Benzo(k)fluoranthene & 360 & $\mathrm{U}$ & 360 & 360 & $\mathrm{U}$ & 360 & 330 & $\mathrm{U}$ & 330 \\
\hline Bis(2-chloro-1-methylethyl)ether & 360 & $\mathrm{U}$ & 360 & 360 & $\mathrm{U}$ & 360 & 330 & $\mathrm{U}$ & 330 \\
\hline Bis(2-chloroethoxy)methane & 360 & $\mathrm{U}$ & 360 & 360 & $U$ & 360 & 330 & $\mathrm{U}$ & 330 \\
\hline Bis(2-chloroethyl) ether & 360 & $\mathrm{U}$ & 360 & 360 & $\mathrm{U}$ & 360 & 330 & $\mathrm{U}$ & 330 \\
\hline Bis(2-ethylhexyl) phthalate & 28 & $\mathrm{JB}$ & 360 & 18 & $\mathrm{~J}$ & 360 & 59 & $\mathrm{~J}$ & 330 \\
\hline Butylbenzylphthalate & 360 & $\mathrm{U}$ & 360 & 360 & $\mathrm{U}$ & 360 & 330 & $\mathrm{U}$ & 330 \\
\hline Carbazole & 360 & $\mathrm{U}$ & 360 & 360 & $\mathrm{U}$ & 360 & 330 & $\mathrm{U}$ & 330 \\
\hline Chrysene & 360 & $\mathrm{U}$ & 360 & 360 & $\mathrm{U}$ & 360 & 330 & $\mathrm{U}$ & 330 \\
\hline Dibenz $(a, h)$ anthracene & 360 & $\mathrm{U}$ & 360 & 360 & $\mathrm{U}$ & 360 & 330 & $\mathrm{U}$ & 330 \\
\hline Dibenzofuran & 360 & $\mathrm{U}$ & 360 & 360 & U & 360 & 330 & $\mathrm{U}$ & 330 \\
\hline Diethylphthalate & 360 & $\mathrm{U}$ & 360 & 360 & $\mathrm{U}$ & 360 & 330 & $\mathrm{U}$ & 330 \\
\hline Dimethylphthalate & 360 & $\mathrm{U}$ & 360 & 360 & $\mathrm{U}$ & 360 & 330 & $\mathrm{U}$ & 330 \\
\hline Di-n-butylphthalate & 360 & $\mathrm{U}$ & 360 & 360 & $\mathrm{U}$ & 360 & 330 & $\mathrm{U}$ & 330 \\
\hline Di-n-octylphthalate & 360 & $\mathrm{U}$ & 360 & 360 & $\mathrm{U}$ & 360 & 330 & $\mathrm{U}$ & 330 \\
\hline Fluoranthene & 360 & $\mathrm{U}$ & 360 & 360 & $\mathrm{U}$ & 360 & 330 & $\mathrm{U}$ & 330 \\
\hline Fluorene & 360 & $\mathrm{U}$ & 360 & 360 & $U$ & 360 & 330 & $\mathrm{U}$ & 330 \\
\hline Hexachlorobenzene & 360 & $\mathrm{U}$ & 360 & 360 & $\mathrm{U}$ & 360 & 330 & $\mathrm{U}$ & 330 \\
\hline Hexachlorobutadiene & 360 & $\mathrm{U}$ & 360 & 360 & $\mathrm{U}$ & 360 & 330 & $\mathrm{U}$ & 330 \\
\hline Hexachlorocyclopentadiene & 360 & $\mathrm{U}$ & 360 & 360 & $\mathrm{U}$ & 360 & 330 & $\mathrm{U}$ & 330 \\
\hline Hexachloroethane & 360 & $\mathrm{U}$ & 360 & 360 & $\mathrm{U}$ & 360 & 330 & $\mathrm{U}$ & 330 \\
\hline Indeno( $1,2,3-c d)$ pyrene & 360 & $\mathrm{U}$ & 360 & 360 & $\mathrm{U}$ & 360 & 330 & $\mathrm{U}$ & 330 \\
\hline Isophorone & 360 & $\mathrm{U}$ & 360 & 360 & $\mathrm{U}$ & 360 & 330 & $\mathrm{U}$ & 330 \\
\hline Naphthalene & 360 & $\mathrm{U}$ & 360 & 360 & $\mathrm{U}$ & 360 & 330 & $\mathrm{U}$ & 330 \\
\hline Nitrobenzene & 360 & $\mathrm{U}$ & 360 & 360 & $\mathrm{U}$ & 360 & 330 & $U$ & 330 \\
\hline N-Nitroso-di-n-dipropylamine & 360 & $\mathrm{U}$ & 360 & 360 & $\mathrm{U}$ & 360 & 330 & $\mathrm{U}$ & 330 \\
\hline N-Nitrosodiphenylamine & 360 & $\mathrm{U}$ & 360 & 360 & $\mathrm{U}$ & 360 & 330 & $\mathrm{U}$ & 330 \\
\hline Pentachlorophenol & 900 & $\mathrm{U}$ & 900 & 900 & $\mathrm{U}$ & 900 & 830 & U & 830 \\
\hline Phenanthrene & 360 & $\mathrm{U}$ & 360 & 360 & $\mathrm{U}$ & 360 & 330 & U & 330 \\
\hline Phenol & 360 & $\mathrm{U}$ & 360 & 360 & $\mathrm{U}$ & 360 & 330 & U & 330 \\
\hline Pyrene & 360 & $\mathrm{U}$ & 360 & 360 & $\mathrm{U}$ & 360 & 330 & $\mathrm{U}$ & 330 \\
\hline & & & Attachment & & 1 & & Sheet No. & & of 18 \\
\hline & & & Originator & $\mathrm{H}$. & Sul & vay & Date & & $03 / 08$ \\
\hline & & & Checked & & D. $\mathrm{Hc}$ & & Date & & $03 / 08$ \\
\hline & & & Calc. No. & 0100 & $\mathrm{CA}-$ & 350 & Rev. No. & & 0 \\
\hline
\end{tabular}




\section{CALCULATION COVER SHEET}

Project Title: 100-F Area Field Remediation

Job No. 14655

Area: $100-\mathrm{F}$

Discipline: Environmental

${ }^{*}$ Calculation No: 0100F-CA-V0355

Subject: 120-F-1 Waste Site Cleanup Verification Hazard Quotient and Carcinogenic Risk Calculation

Computer Program: Excel

Program No: Excel 2003

The attached calculations have been generated to document compliance with established cleanup levels. These calculations should be used in conjunction with other relevant documents in the administrative record.

Committed Calculation $\bar{X}$

Preliminary $\Gamma$

Superseded $\Gamma$

Voided $\Gamma$

\begin{tabular}{|c|c|c|c|c|c|c|}
\hline Rev. & Sheet Numbers & Originator & Checker & Reviewer & Approval & Date \\
\hline 0 & $\begin{array}{c}\text { Cover }=1 \\
\text { Summary }=3\end{array}$ & H. M. Sulloway & L. D. Habel & & J. D. Fancher & signed $4 / 2 / 08$ \\
\hline 1 & $\begin{array}{c}\text { Cover }=1 \\
\text { Summary }=3\end{array}$ & H. M. Sulloway & & $N A$ & J.P. Fankcher & $5 / 216$ \\
\hline & & & & & & \\
\hline & & & & & & \\
\hline & & & & & $\therefore$ & \\
\hline & & & & & & \\
\hline
\end{tabular}

SUMMARY OF REVISION

\begin{tabular}{|l|l|}
\hline 1 & $\begin{array}{l}\text { Entire calculation revised for simplicity. Changes are as follows: } 1 . \text { Sheet 2, line } 26 \text { and } 27, \text { the term } \\
\text { 'statistical' replaced with the term maximum as value was not obtained from a statistical calculation, } 2 . \\
\text { sheet 3, line 5, column heading term "Maximum" changed to "Statistical or Maximum Result", 3. sheet } 3, \\
\text { line 13, added fluoride to hazard quotient calculation, and 4. sheet 3, line 19, value of 4,4'-DDT changed } \\
\text { from } 0.0017 \text { to } 0.0021 .\end{array}$ \\
\hline & \\
\hline
\end{tabular}


Washington Closure Hanford, Inc. CALCULATION SHEET

\begin{tabular}{|c|c|c|c|c|c|c|c|}
\hline Origina & H. M. Sulloway dflus & Date: & $05 /$ & Calc. No.: & $0100 \mathrm{~F}-\mathrm{C} A$ & Re & 1 \\
\hline & 100-F Area Field Remediation & Job No: & 14655 & Checked: & L. D. Habelotetit & Da & $05 /$ \\
\hline & \multicolumn{7}{|c|}{ 120-F-1 Cleanup Verification Hazard Quotient and Carcinogenic Risk Calculation } \\
\hline
\end{tabular}

\section{PURPOSE:}

Provide documentation to support the calculation of the hazard quotient (HQ) and carcinogenic (excess cancer) risk for the 120-F-1 glass dump waste site. In accordance with the remedial action goals (RAGs) in the remedial design report/remedial action work plan (RDR/RAWP) (DOE-RL 2005), the following criteria must be met:

1) An HQ of $<1.0$ for all individual noncarcinogens

2) A cumulative $\mathrm{HQ}$ of $<1.0$ for noncarcinogens

3) An excess cancer risk of $<1 \times 10^{-6}$ for individual carcinogens

4) A cumulative excess cancer risk of $<1 \times 10^{-5}$ for carcinogens.

\section{GIVEN/REFERENCES:}

1) Capron, J. M., 2008, Revised 120-F-1 Verification Sampling, CCN 138678, email to R. Lobos (EPA) and C. Smith (DOE), dated February 14, 2008, Washington Closure Hanford, Inc., Richland, Washington.

2) DOE-RL, 2005, Remedial Design Report/Remedial Action Work Plan for the 100 Areas, DOE/RL-96-17, Rev. 5, U.S. Department of Energy, Richland Operations Office, Richland, Washington.

3) WAC 173-340, "Model Toxics Control Act - Cleanup," Washington Administrative Code, 1996.

4) WCH, 2007, Work Instruction for Verification Sampling of the 120-F-1 Glass Dump Waste Site, Work Instruction No. 0100F-WI-G0069, Washington Closure Hanford, Inc., Richland, Washington.

5) WCH, 2008, 120-F-1 Cleanup Verification 95\% UCL Calculation, Calc. No. 0100F-CA-V0345, Washington Closure Hanford, Inc., Richland, Washington.

\section{SOLUTION:}

1) Generate an HQ for each noncarcinogenic constituent detected above background or required detection limit/practical quantitation limit and compare it to the individual HQ of $<1.0$ (DOE-RL 2005).

2) Sum the HQs and compare this value to the cumulative HQ of $<1.0$.

3) Generate an excess cancer risk value for each carcinogenic constituent detected above background or required detection limit/practical quantitation limit and compare it to the excess cancer risk of $<1 \times 10^{-6}$ (DOE-RL 2005).

4) Sum the excess cancer risk value(s) and compare it to the cumulative cancer risk of $<1 \times 10^{-5}$. 
Washington Closure Hanford, Inc.

CALCULATION SHEET

\begin{tabular}{r|r|r|r|r|r|r|r|} 
Project: & $100-\mathrm{F}$ Area Field Remediation & Job No: & 14655 & Checked: & L. D. Habel $h \psi$ & Date: & $05 / 06 / 08$ \\
\hline
\end{tabular}

\begin{tabular}{l|l|l|l} 
Subject: & $120-\mathrm{F}-1$ Cleanup Verification Hazard Quotient and Carcinogenic Risk Calculation & Sheet No. 2 of 3
\end{tabular}

\section{METHODOLOGY:}

The 120-F-1 waste site was divided into two sampling areas (northwest and southwest excavations) for verification sampling (WCH 2007). The original area of the northwest excavation was expanded after the original sampling showed additional soil removal would be required. The sample design for the northwest area was updated and approved by the regulators (Capron 2008). The maximum values from the combined results of the two sampling areas were used in developing the HQ and risk calculations. Of the nonradionuclide contaminants of potential concern (COPCs), mercury and selenium required the HQ and risk calculations because they were quantified above background. Boron, molybdenum, and hexavalent chromium values require HQ and risk calculations because these analytes were detected and a Washington State or Hanford Site background value is not available. Aroclor-1254, Aroclor-1260, and multiple organic COPCs (as listed in Table 1) are included because they were detected by laboratory analysis and cannot be attributed to natural occurrence. All other site nonradionuclide COCs were not detected or were quantified below background levels.

An example of the HQ and risk calculations is presented below:

1) For example, the statistical value for boron is $4.6 \mathrm{mg} / \mathrm{kg}$, divided by the noncarcinogenic RAG value of $16,000 \mathrm{mg} / \mathrm{kg}$ (boron is identified as a noncarcinogen in WAC 173-340-740[3]), is $2.9 \times 10^{-4}$. Comparing this value to the requirement of $<1.0$, this criteria is met.

2) After the HQ calculation is completed for the appropriate analytes, the cumulative HQ can be obtained by summing the individual values. The sum of the HQ values (shown in Table 1) is $4.2 \times 10^{-2}$. Comparing this value to the requirement of $<1.0$, this criteria is met.

3) To calculate the excess cancer risk, the maximum value is divided by the carcinogenic RAG value, then multiplied by $1 \times 10^{-6}$. For example, the maximum value for hexavalent chromium is $1.8 \mathrm{mg} / \mathrm{kg}$, divided by $2.1 \mathrm{mg} / \mathrm{kg}$, and multiplied as indicated, is $8.6 \times 10^{-7}$. Comparing this value and all other individual values to the requirement of $<1 \times 10^{-6}$, this criteria is met.

4) After these calculations are completed for the carcinogenic analytes, the cumulative excess cancer risk can be obtained by summing the individual values. The sum of the excess cancer risk values is $1.1 \times 10^{-6}$. Comparing this value to the requirement of $<1 \times 10^{-5}$, this criterion is met.

\section{RESULTS:}

1) List individual noncarcinogens and corresponding HQs $>1.0$ : None

2) List the cumulative noncarcinogenic $H Q>1.0$ : None

3) List individual carcinogens and corresponding excess cancer risk $>1 \times 10^{-6}$ : None

4) List the cumulative excess cancer risk for carcinogens $>1 \times 10^{-5}$ : None.

Table 1 shows the results of the calculations. 
Washington Closure Hanford, linc.

CALCULATION SHEET

\begin{tabular}{l|l|l|l|l|l|r|r|r} 
& \\
\hline
\end{tabular}

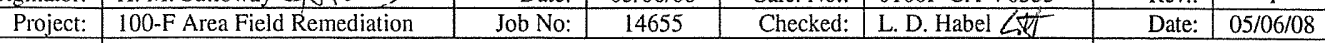

\begin{tabular}{l|l|l} 
Subject: & $120-\mathrm{F}-1$ Cleanup Verification Hazard Quotient and Carcinogenic Risk Calculation & Sheet No. 3 of 3
\end{tabular}

4

5

6

7

8

10

11

12

13

14

15

16

17

18

19

20

21

22

23

24

25

26

27

Table 1. Hazard Quotient and Excess Cancer Risk Results for the 120-F-1 Waste Site.

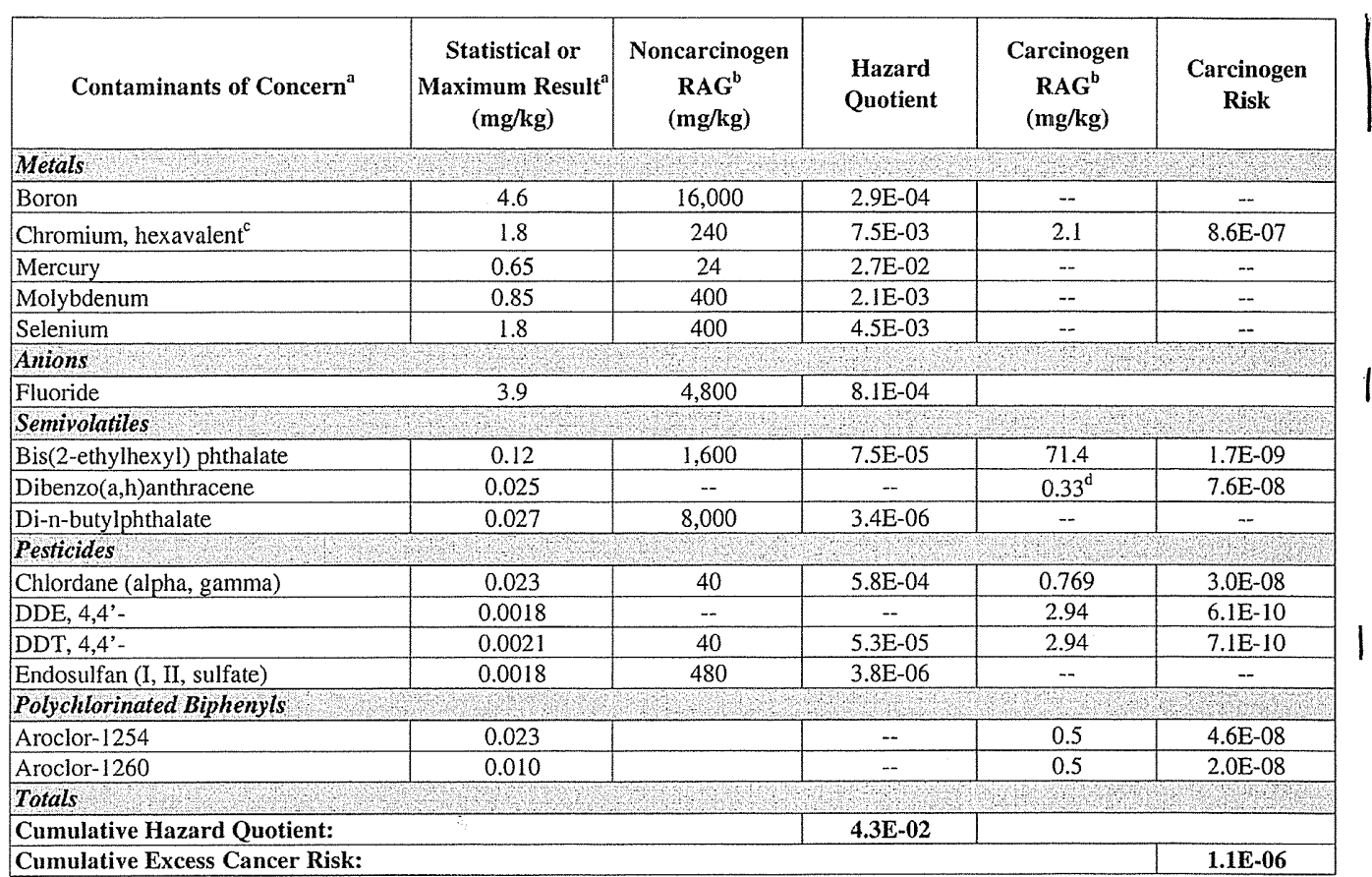

Notes:

" = From WCH (2008)

${ }^{\mathrm{h}}=$ Value obtained from the RDR/RAWP (DOE-RL 2005) or Washington Administrative Code (WAC) 173-340-740(3), Method B, 1996, unless otherwise noted.

${ }^{c}=$ Value for the carcinogen RAG calculated based on the inhalation exposure pathway WAC 173-340-750(3), 1996.

"Individual carcinogenic risk calculated using the required detection limit. Contribution to cumulative carcinogenic risk calculated based on the remedial action goal instead of the required detection limit, per WAC 173-340-740(3), Method B, 1996.

-. = not applicable

$\mathrm{RAG}=$ remedial action goal

\section{CONCLUSION:}

This calculation demonstrates that the $120-\mathrm{F}-1$ waste site meets the requirements for the hazard quotients and carcinogenic (excess cancer) risk as identified in the RDR/RAWP (DOE-RL 2005). 


\section{APPENDIX D}

\section{SUMMARY OF UPDATED SAMPLE DESIGN FOR 120-F-1 NORTHWEST EXCAVATION}




\section{Summary}

This appendix summarizes the updated sampling design used and associated statistical assumptions for verification sampling of the northwest excavation of 120-F-1 site, as well as general guidelines to be used for conducting post-sampling data analysis. Sampling plan components presented here include how many sampling locations to choose and where, within the sampling area, to collect those samples. Requirements for collecting and analyzing the samples are provided in (WCH 2007).

\section{Primary Sampling Objective}

The primary purpose of sampling at this site is to compare a site mean value with a fixed threshold. The decision rule for demonstrating compliance with the cleanup criteria requires comparison of the true population mean, as estimated by the $95 \%$ upper confidence limit on the sample mean, with the cleanup level (DOE-RL 2005). The working hypothesis (or "null" hypothesis) is that the mean value at the site is equal to or exceeds the action threshold (the site is "dirty"). The alternative hypothesis is that the mean value is less than the threshold. Visual Sample Plan (VSP) calculates the number of samples required to reject the null hypothesis in favor of the alternative one, given a selected sampling approach and inputs to the associated equation.

\section{Selected Sampling Approach}

A nonparametric, systematic sampling approach with a random start was used to determine the number of samples and to specify sampling locations. A nonparametric formula was selected because the site conceptual model and analogous information (i.e., data from similar sites) indicate that typical parametric assumptions may not be true.

Both parametric and nonparametric equations rely on assumptions about the population. Typically, however, nonparametric equations require fewer assumptions and allow for more uncertainty about the statistical distribution of values at the site. Alternatively, if the parametric assumptions are valid, the required number of samples is usually less than if a nonparametric equation was used.

The Washington State Department of Ecology publication Guidance on Sampling and Data Analysis Methods (Ecology 1995) recommends that systematic sampling with sample locations distributed over the entire study area be used. Therefore, a systematic grid sampling design with a random start was selected for use in VSP. Locating the sample points over a systematic grid with a random start ensures spatial coverage of the site. Statistical analyses of systematically collected data are valid if a random start to the grid is used. One disadvantage of systematically

\footnotetext{
${ }^{1}$ Visual Sample Plan is a site map-based user-interface program that may be downloaded at http://dqo.pnl.gov.
} 
collected samples is that spatial variability or patterns may not be discovered if the grid spacing is large relative to the spatial patterns.

\section{Number of Total Samples: Calculation Equation and Inputs}

The equation used to calculate the number of samples is based on a Sign test (see Gilbert et al. 2001 for discussion). For this site, the null hypothesis is rejected in favor of the alternative, if the mean is sufficiently smaller than the threshold. The number of samples to collect is calculated such that, if the inputs to the equation are true, the calculated number of samples will cause the null hypothesis to be rejected.

The formula used to calculate the number of samples is as follows:

$$
n=1.20\left[\frac{\left(Z_{1-\alpha}+Z_{1-\beta}\right)^{2}}{4(\operatorname{Sign} P-0.5)^{2}}\right]
$$

where:

$$
\operatorname{Sign} P=\Phi\left(\frac{\Delta}{s_{\text {Total }}}\right)
$$

$\Phi(z)=$ the cumulative standard normal distribution on $(-\infty, \mathrm{z})$ (see Gilbert et al. 2001 for details)

$n=$ the number of samples

$S=$ the estimated standard deviation of the measured values including analytical error

$\Delta \quad=$ the width of the gray region

$\alpha=$ the acceptable probability of incorrectly concluding the site mean is less than the threshold

$\beta=$ the acceptable probability of incorrectly concluding the site mean exceeds the threshold

$Z_{1-\alpha}=$ the value of the standard normal distribution such that the proportion of the distribution less than $Z_{1-\alpha}$ is $1-\alpha$

$Z_{1-\beta}=$ the value of the standard normal distribution such that the proportion of the distribution less than $Z_{1-\beta}$ is $1-\beta$.

NOTE: The Multi-Agency Radiation Survey and Site Investigation Manual (MARSSIM)

(EPA et al. 2000) suggests that the number of samples should be increased by at least $20 \%$ to account for missing or unusable data and uncertainty in the calculated value of $n$. VSP allows a user-supplied percent overage as discussed in MARSSIM (EPA et al. 2000, p. 5-33).

The values of these inputs that result in the calculated number of sampling locations are summarized in Table C-1. 
Table D-1. VSP User Inputs.

\begin{tabular}{|l|c|l|}
\hline \multicolumn{1}{|c|}{ Parameter } & Value & \multicolumn{1}{c|}{ Basis } \\
\hline$S$ & 0.25 & Assumed standard deviation. \\
\hline$\Delta$ & 0.45 & User defined conservative value. \\
\hline$\alpha$ & $5 \%$ & False rejection rate specified in the DQO summary report (BHI 2003). \\
\hline$\beta$ & $20 \%$ & False acceptance rate specified in the DQO summary report (BHI 2003). \\
\hline$Z_{1-\alpha}$ & 1.64485 & $\begin{array}{l}\text { This value is automatically calculated by VSP based on the user-defined } \\
\text { value of } \alpha .\end{array}$ \\
\hline$Z_{1-\beta}$ & 0.841621 & $\begin{array}{l}\text { This value is automatically calculated by VSP based on the user-defined } \\
\text { value of } \beta .\end{array}$ \\
\hline MARSSIM overage & $20 \%$ & User-defined sample increase factor. \\
\hline
\end{tabular}

DQO = data quality objective

MARSSIM = Multi-Agency Radiation Survey and Site Investigation Manual

VSP $\quad=$ Visual Sample Plan

In order to use VSP to calculate the appropriate number of samples, $n$, to collect for estimating the mean, it is necessary to have some estimate of the sample standard deviation. In general, estimates made from samples tend to more closely approximate the true population mean as the number of samples increases. Appropriate statistical parameters (i.e., standard deviation within the population) for the post-remediation residual contaminant concentration levels at the 120-F-1 waste site are unknown. For the purpose of the development of the statistical sampling design, a generic action limit of 1.0 can be assumed (where 0.5 would be $50 \%$ of the action limit). The standard deviation for each residual contaminant population was then conservatively assumed to be less than $25 \%$ of the corresponding decision threshold for the population. Using this standard deviation value along with a conservative "gray region" (45\% or 0.45$)$ in VSP, the estimated number of verification samples to collect is calculated. These assumptions will be verified in the data quality assessment using verification sampling data from the resulting data set.

Table D-2 summarizes the sampling design that was developed. Table D-3 lists sampling location coordinates. Figure D-1 shows sampling locations in the field.

Table D-2. Summary of Sampling Design.

\begin{tabular}{|l|l|}
\hline Primary objective of design & Compare a site mean to a fixed threshold \\
\hline Type of sampling design & Nonparametric \\
\hline Sample placement (location) in the field & Systematic with a random start location \\
\hline Working (null) hypothesis & The mean value at the site exceeds the threshold \\
\hline $\begin{array}{l}\text { Formula for calculating number of sampling } \\
\text { locations }\end{array}$ & Sign test - MARSSIM version \\
\hline Calculated total number of samples & 10 per sampling area \\
\hline Specified sampling area & $322.3 \mathrm{~m}^{2}\left(3468.8 \mathrm{ft}^{2}\right)$ \\
\hline Size of grid/area of grid cell & b.1 $\mathrm{m}(20.0 \mathrm{ft}) / 32.2 \mathrm{~m}^{2}\left(346.9 \mathrm{ft}^{2}\right)$ \\
\hline Grid pattern & Triangular \\
\hline
\end{tabular}

a The sampling area is the total surface area of the selected shaded sample areas on the map of the site.

${ }^{b}$ Size of grid/area of grid cell gives the linear distance between grid points and the grid cell area used to systematically place samples.

MARSSIM = Multi-Agency Radiation Survey and Site Investigation Manual 
Table D-3. Verification Sample Location Coordinates.

\begin{tabular}{|c|c|c|c|}
\hline X Coord & Y Coord & Label & Type \\
\hline 581035.4 & 147211.5 & 1 & Systematic \\
\hline 581041.3 & 147210.0 & 2 & Systematic \\
\hline 581033.8 & 147217.4 & 3 & Systematic \\
\hline 581039.7 & 147215.9 & 4 & Systematic \\
\hline 581045.6 & 147211.3 & 5 & Systematic \\
\hline 581051.5 & 147212.7 & 6 & Systematic \\
\hline 581038.1 & 147221.8 & 7 & Systematic \\
\hline 581044.0 & 147220.2 & 8 & Systematic \\
\hline 581049.9 & 147218.6 & 9 & Systematic \\
\hline 581055.8 & 147217.1 & 10 & Systematic \\
\hline
\end{tabular}


Figure D-1. Map of 120-F-1 Northwest Excavation Verification Sample Locations.

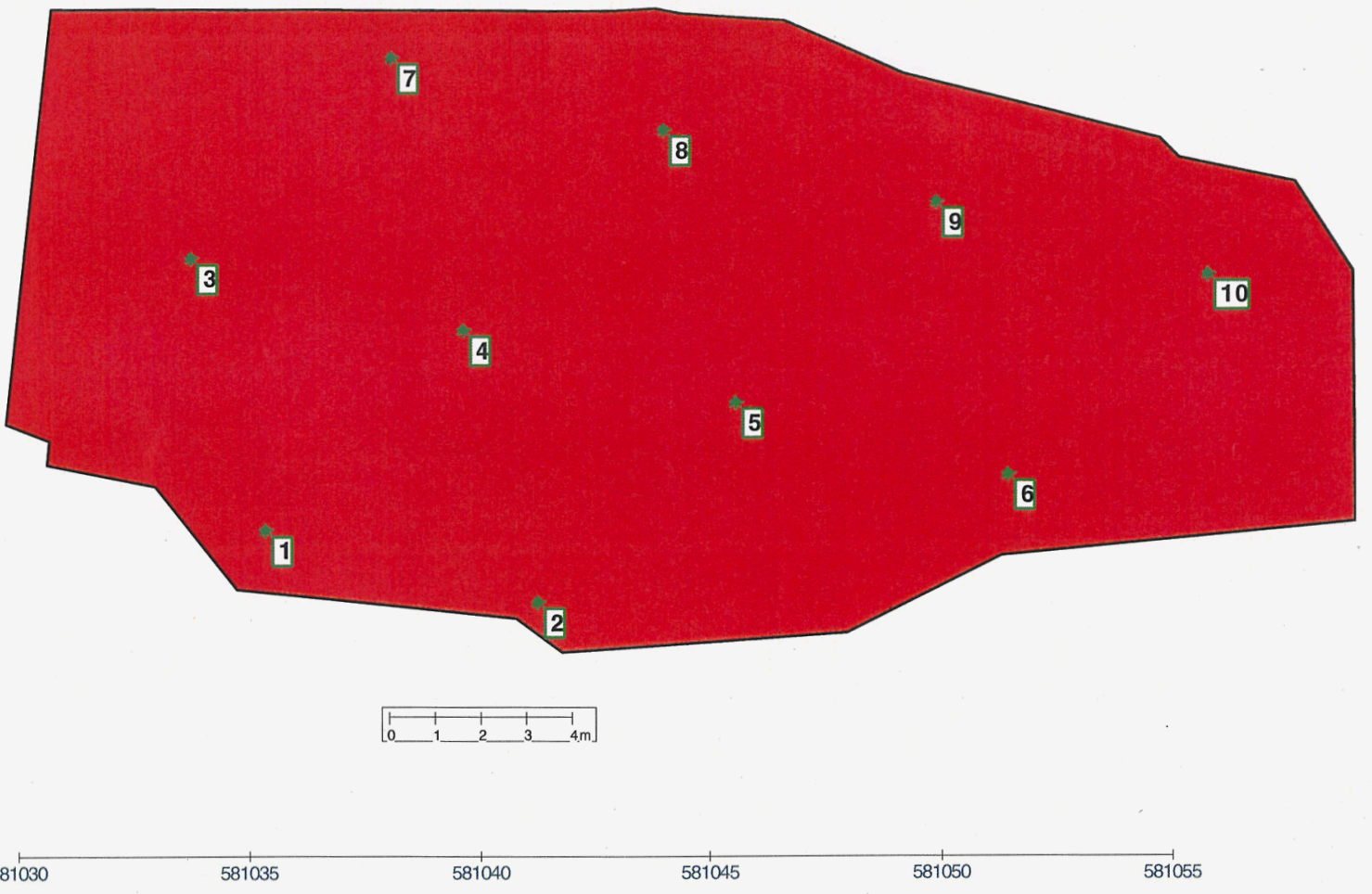

Figure D-2 is a performance goal diagram, described in the U.S. Environmental Protection Agency's QA/G-4 guidance (EPA 2000b). This figure shows the probability of concluding that the sample area is dirty on the vertical axis versus a range of possible unit true median (mean) values (where 0.5 would be $50 \%$ of the action limit for a specific contaminant of concern) for the site on the horizontal axis. These graphs contain all of the inputs to the number of samples equation and pictorially represent the calculations.

The solid vertical line to the right of the gray region is shown at the threshold (unit action limit) on the horizontal axis. The width of the gray shaded area is equal to $\Delta$; the upper horizontal dashed line is positioned at $1-\alpha$ on the vertical axis; and the lower horizontal dashed line is positioned at $\beta$ on the vertical axis. The short vertical line in the gray region to the left of the action level is positioned at one standard deviation below the threshold. The shape of the curve corresponds to the estimates of variability. The calculated number of samples results in the curve that passes through the lower bound of $\Delta$ at $\beta$ and the upper bound of $\Delta$ at 1- $\alpha$. 
Figure D-2. Performance Goal Diagram.

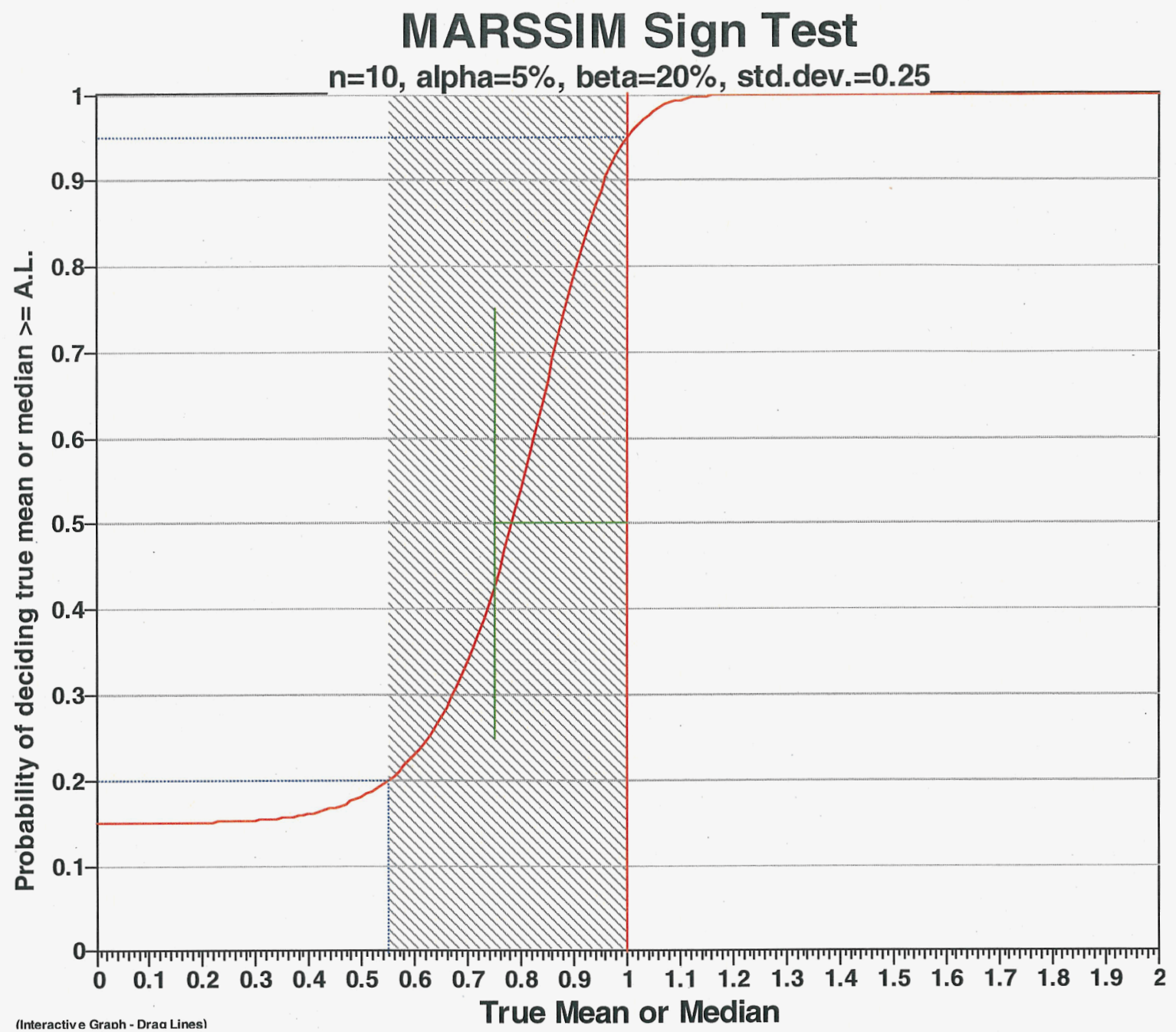

\section{Statistical Assumptions}

The assumptions associated with the formulae for computing the number of samples are as follows:

1. The computed Sign test statistic is normally distributed.

2. The variance estimate, $S^{2}$, is reasonable and representative of the population being sampled.

3. The population values are not spatially or temporally correlated.

4. The sampling locations will be selected probabilistically.

The first three assumptions will be assessed in a post-data collection analysis. The last assumption is valid because the gridded sample locations were selected based on a random start. 


\section{Sensitivity Analysis}

The sensitivity of the calculation of number of samples was explored by varying $S$, lower bound of the gray region (LBGR), $\beta$ and $\alpha$ and examining the resulting changes in the number of samples. Table D-4 shows the results of this analysis.

Table D-4. Sensitivity Analysis.

\begin{tabular}{|c|c|c|c|c|c|c|c|}
\hline \multicolumn{9}{|c|}{} & \multicolumn{2}{c|}{$\alpha=\mathbf{5}$} & \multicolumn{2}{c|}{$\alpha=\mathbf{1 0}$} & \multicolumn{2}{c|}{$\alpha=\mathbf{1 5}$} \\
\hline \multicolumn{2}{|c|}{$\mathbf{A L = 1}$} & $S=\mathbf{0 . 5}$ & $S=\mathbf{0 . 2 5}$ & $S=\mathbf{0 . 5}$ & $S=\mathbf{0 . 2 5}$ & $\boldsymbol{S = 0 . 5}$ & $S=\mathbf{0 . 2 5}$ \\
\hline LBGR=90 & $\beta=\mathbf{1 5}$ & 345 & 90 & 257 & 68 & 206 & 54 \\
\hline & $\beta=\mathbf{2 0}$ & 297 & 77 & 216 & 57 & 170 & 45 \\
\hline & $\beta=\mathbf{2 5}$ & 258 & 68 & 184 & 48 & 141 & 38 \\
\hline LBGR=80 & $\beta=\mathbf{1 5}$ & 90 & 27 & 68 & 21 & 54 & 16 \\
\hline & $\beta=\mathbf{2 0}$ & 77 & 23 & 57 & 17 & 45 & 14 \\
\hline & $\beta=\mathbf{2 5}$ & 68 & 21 & 48 & 15 & 38 & 11 \\
\hline LBGR=70 & $\beta=\mathbf{1 5}$ & 44 & 16 & 33 & 12 & 27 & 10 \\
\hline & $\beta=\mathbf{2 0}$ & 38 & 14 & 28 & 10 & 22 & 8 \\
\hline & $\beta=\mathbf{2 5}$ & 33 & 12 & 23 & 9 & 18 & 6 \\
\hline
\end{tabular}

$\mathrm{LBGR}=$ lower bound of the gray region

\section{Recommended Data Analysis Activities}

Post-data collection activities generally follow those outlined in the U.S. Environmental Protection Agency's Guidance for Data Quality Assessment (EPA 2000a). The data analysts will become familiar with the context of the problem and goals for data collection and assessment. The data will be verified and validated before being subjected to statistical or other analyses. Graphical and analytical tools will be used to verify, to the extent possible, the assumptions of any statistical analyses that are performed as well as to achieve a general understanding of the data. The data will be assessed to determine if they are adequate in both quality and quantity to support the primary objective of sampling.

Because the primary objective for sampling for this site is to compare the site mean values with threshold values, the data will be assessed in this context. Assuming the data are adequate, statistical tests will be done, as necessary, to perform a comparison between the data and the threshold(s) of interest. Results of the exploratory and quantitative assessments of the data will be reported, along with conclusions that may be supported by them. 


\section{References}

BHI, 2003, Data Quality Objectives Summary Report for 100/300 Area Remaining Sites Analytical Sampling Effort, BHI-01249, Rev. 3, Bechtel Hanford, Inc., Richland, Washington.

DOE-RL, 2005, Remedial Design Report/Remedial Action Work Plan for the 100 Area, DOE/RL-96-17, Rev. 5, U.S. Department of Energy, Richland Operations Office, Richland, Washington.

Ecology, 1995, Guidance on Sampling and Data Analysis Methods, Publication No. 94-49, Washington State Department of Ecology, Olympia, Washington.

EPA, 2000a, Guidance for Data Quality Assessment: Practical Methods for Data Analysis, EPA/600/R-96/084, U.S. Environmental Protection Agency, Washington, D.C.

EPA, 2000b, Guidance for the Data Quality Objectives Process, EPA QA/G-4, EPA/600/R-96/055, U.S. Environmental Protection Agency, Washington, D.C.

EPA, DOD, DOE, and NRC, 2000, Multi-Agency Radiation Survey and Site Investigation Manual (MARSSIM), NUREG-1575, Rev. 1, EPA 402-R-97-016, Rev. 1, DOE/EH-0624, Rev. 1, U.S. Environmental Protection Agency, U.S. Department of Defense, U.S. Department of Energy, and U.S. Nuclear Regulatory Commission, Washington, D.C.

Gilbert R. O., J. R. Davidson, J. E. Wilson, and B. A. Pulsipher, 2001, Visual Sample Plan (VSP) Models and Code Verification, PNNL-13450, Pacific Northwest National Laboratory, Richland, Washington.

WCH, 2007, Work Instruction for Verification Sampling of the 120-F-1 Glass Dump Waste Site, 0100F-WI-G0069, Rev. 0, Washington Closure Hanford, Richland, Washington. 


\section{Verification Sampling Data Quality Assessment}

A data quality assessment (DQA) was performed to compare the verification sampling approach and resulting analytical data with the sampling and data requirements specified in the sitespecific sample design (DOE-RL 2005a, WHC 2007, WCH 2008a). This DQA was performed in accordance with site specific data quality objectives found in the SAP (DOE-RL 2005b).

A review of the sample design (WCH 2007, WCH 2008a), the field logbook (WCH 2008b), and applicable analytical data packages has been performed as part of this DQA. All samples were collected per the sample design. In addition, ion chromatography (IC) anions, sulfides, cyanide, and $\mathrm{pH}$ analyses were performed on the verification samples collected at the 120-F-1 waste site. These constiuents are not contaminants of concern (COCs) for the 120-F-1 waste site. This DQA limited the data review for the 120-F-1 verification sampling to the data required per the sample design.

To ensure quality data, the SAP data assurance requirements and the data validation procedures for chemical analysis (BHI 2000) are used as appropriate. This review involves evaluation of the data to determine if they are of the right type, quality, and quantity to support the intended use (i.e., closeout decisions). The DQA completes the data life cycle (i.e., planning, implementation, and assessment) that was initiated by the data quality objectives process (EPA 2000).

The closeout sampling approach for the 120-F-1 Glass Dump waste site included a sample design with multiple subunit areas. Verification sample data collected at the 120-F-1 waste site were provided by the laboratories in four sample delivery groups (SDGs). For the 120-F-1 southeastern dump site, verification sample data was provided in SDG K1066, SDG K1067, and SDG K1134. SDG K1066 was submitted for third-party validation. For the 120-F-1 northwestern dump site, verification sample data was provided in SDG K1155. No major deficiencies were found in the in the DQA review of the analytical data set. Minor deficiencies found in the analytical data set are discussed below.

\section{SDG K1066}

This SDG comprises six verification samples (J16337-J16342) collected from the southeastern dump site. These samples were analyzed for inductively coupled plasma (ICP) metals, mercury, semivolatile organic compounds (SVOCs), pesticides, polychlorinated biphenyls (PCBs), and total petroleum hydrocarbon (TPH). Anions, sulfides, cyanide, and $\mathrm{pH}$ analyses were also performed for these samples; however, these constituents are outside the scope of the sample design and are not included in this DQA review. In addition, one equipment blank (J16354) was collected and analyzed for ICP metals and mercury. SDG K1066 was submitted for formal third-party validation. No major deficiencies were found in SDG K1066. No major deficiencies were found in SDG K1066. Minor deficiencies are as follows:

In the SVOC analysis, the common laboratory contaminants bis(2-ethylhexyl)phthalate are detected in the method blank (MB). Third party validation raised the reported values for bis(2-ethylhexyl)phthalate for all samples to the required quantitation limit of $660 \mu \mathrm{g} / \mathrm{kg}$ and 
qualified them as undetected and flagged "U". The data are useable for decision-making purposes.

In the SVOC analysis, the matrix spike (MS) and matrix spike duplicate (MSD) recoveries are below the acceptance criteria for 2,2'-oxybis(1-chloropropane), at $49 \%$ and $45 \%$, respectively. The laboratory control sample (LCS) recovery was outside QC limits for 2,4-dinitrophenol (28\%), 4,6-dinitro-2-methylphenol (47\%), and pentachlorophenol (48\%). All results for analytes with low MS or LCS recoveries were qualified as estimates and flagged "J" by third party validation. Estimated data are useable for decision making purposes.

The relative percent difference (RPD) for 2,4-dinitrophenol (56\%) is greater than 30\%. The results for 2,4-dinitrophenol in all samples were qualified as estimates and flagged "J" by third party validation. Estimated data are useable for decision making purposes.

The MS and MSD samples (J16337 MS and MSD) for the pesticide and PCB analyses in SDG K1066 were prepared in separate preparation batches. As a result, all pesticide and PCB results for samples J16338-J16342 were qualified by third-party validation as estimated with "J" flags. Estimated, or "J"-flagged, data are acceptable for decision making purposes.

All of the toxaphene data in SDG K1066 was qualified by third-party validation as estimated with "J" flags, due to lack of a MS, MSD, or LCS analysis for the analyte. Estimated, or "J" flagged, data are acceptable for decision making purposes.

In the ICP metals analysis, the calcium, sodium, and zinc results for sample J16354 (the equipment blank) are of similar magnitude as the method blank results. These results are qualified by third party validation as undetected estimates with "UJ" flags, due to method blank contamination. The data are useable for decision-making purposes.

Also in the ICP metals analysis, the MS recoveries for three ICP metals (aluminum, iron, and silicon) are out of acceptance criteria. For these analytes, the spiking concentration is insignificant compared to the native concentration in the sample from which the MS was prepared. Therefore, the deficiency in the MS result is a reflection of the analytical variability of the native concentration rather than a measure of the recovery from the sample. To confirm quantitation, post digestion spikes (PDSs) and serial dilutions were prepared for all three analytes with acceptable results. The data are useable for decision making purposes.

Also in the ICP metals analysis, the laboratory duplicate RPD for boron is above the acceptance criteria at $98.7 \%$. Elevated RPDs in environmental soil samples are generally attributed to heterogeneities in the sample matrix and not to deficiencies in the laboratory procedures. The data are useable for decision making purposes.

\section{SDG K1067}

This SDG comprises five verification samples (J16337-J16342) collected from the southeastern dump site. A field duplicate pair (J16333/J16334) is included in this SDG. These samples were analyzed for ICP metals, mercury, SVOCs, pesticides, PCBs, and TPH. Anions, sulfides, 
cyanide, and $\mathrm{pH}$ analyses were also performed for these samples; however, these constituents are outside the scope of the sample design and are not included in this DQA review. No major deficiencies were found in SDG K1067. Minor deficiencies are as follows:

In the ICP metals analysis, the MS recoveries for three ICP metals (aluminum, iron, and silicon) are out of acceptance criteria. For these analytes, the spiking concentration is insignificant compared to the native concentration in the sample from which the MS was prepared. Therefore, the deficiency in the MS result is a reflection of the analytical variability of the native concentration rather than a measure of the recovery from the sample. To confirm quantitation, PDSs and serial dilutions were prepared for all three analytes with acceptable results. The data are useable for decision making purposes.

Also in the ICP metals analysis, the laboratory duplicate RPD for boron is above the acceptance criteria at $90.9 \%$. Elevated RPDs in environmental soil samples are generally attributed to heterogeneities in the sample matrix and not to deficiencies in the laboratory procedures. The data are useable for decision making purposes.

All of the toxaphene data in SDG K1067 is considered estimated due to lack of a MS, MSD, or LCS analysis for the analyte. Estimated data are acceptable for decision making purposes.

In the SVOC analysis, 3 of $128 \mathrm{MS}$ recoveries are outside the acceptance criteria. The MSD recoveries are below the acceptance criteria for 2,2'-oxybis(1-chloropropane) and 4,6-dinitro-2methylphenol, at $45 \%$ and $37 \%$ respectively. The MS recovery was below QC limits for pentachlorophenol (29\%). All results for analytes with low MS recoveries are considered estimated. Estimated data are useable for decision making purposes.

\section{SDG $\mathbb{K} 1134$}

This SDG comprises eleven verification samples (J16B36-J16B46) collected from the southeastern dump site. A field duplicate pair (J16B45/J16B46) is included in this SDG. These samples were all analyzed for hexavalent chromium. No major deficiencies were found in SDG K1134. Minor deficiencies are as follows:

The laboratory duplicate RPD for hexavalent chromium is above the acceptance criteria at $35.4 \%$. Elevated RPDs in environmental soil samples are generally attributed to heterogeneities in the sample matrix and not to deficiencies in the laboratory procedures. The data are useable for decision making purposes.

\section{SDG K1155}

This SDG comprises eleven verification samples (J16DT7-J16DT9 and J16DV0-J16DV7) collected from the northwestern dump site. A field duplicate pair (J16DV7/J16DV6) is included in this SDG. These samples were analyzed for ICP metals, mercury, hexavalent chromium, SVOCs, pesticides, PCBs, and TPH. Anions, sulfides, cyanide, and pH analyses were also performed for these samples; however, these constituents are outside the scope of the sample 
design and are not included in this DQA review. In addition, one equipment blank (J16DT6) was collected and analyzed for ICP metals and mercury. Major and minor deficiencies are as follows:

All of the toxaphene data in SDG K1155 is considered estimated due to lack of a MS, MSD, or LCS analysis for the analyte. Estimated data are acceptable for decision making purposes.

Six of 128 MS recoveries in the SVOC analysis are below the acceptance criteria. The MS and MSD recoveries for 4-chloro-3-methylphenol are 55\% and 58\%, respectively. The MS recoveries for nitrobenzene and isophorone are $47 \%$ and $56 \%$, respectively. The MS for 1,2,4-trichlorobenzene is $51 \%$ and the MS for 2 -methylnaphthalene is $57 \%$. Six LCS recoveries were outside QC limits. The LCS recoveries for isophorone, 1,2,4-trichlorobenzene, and 4chloro-3-methylphenol, are 59\%, 57\%, and 56\%, respectively. The LCS recoveries for 2-methylnaphthalene, 2,4-dinitrophenol, and 4,6-dinitro-2-methylphenol, are 58\%, 16\%, and $28 \%$, respectively. The results for these analytes may be considered estimated. Estimated data are useable for decision making purposes.

In the ICP metals analysis, the MS recoveries for four ICP metals (aluminum, iron, antimony, and silicon) are out of acceptance criteria. For most of these analytes, the spiking concentration is insignificant compared to the native concentration in the sample from which the MS was prepared. Therefore, the deficiency in the MS result is a reflection of the analytical variability of the native concentration rather than a measure of the recovery from the sample. To confirm quantitation, PDSs and serial dilutions were prepared for the analytes with acceptable results. Antimony did not have mismatched spike and native concentrations in the original MS. The original MS recovery for antimony was $54.7 \%$. The antimony results in SDG K1155 may be considered estimated. Estimated data are useable for decision making purposes.

\section{FIELD QUALITY ASSURANCE/QUALITY CONTROL}

RPD evaluations of main sample(s) versus the laboratory duplicate(s) are routinely performed and reported by the laboratory. Any deficiencies in those calculations are reported by SDG in the previous sections.

Field quality assurance/ quality control (QA/QC) measures are used to assess potential sources of error and cross contamination of samples that could bias results. Field QA/QC samples, listed in the field logbook (WCH 2008b), are summarized in Table 1. The main and QA/QC sample results are presented in Appendix C. 
Table 1. Field Quality Assurance/Quality Control Samples.

\begin{tabular}{|c|c|c|}
\hline Sample Area & Main Sample & $\begin{array}{c}\text { Duplicate } \\
\text { Sample }\end{array}$ \\
\hline Southeastern dump site & J16333 & J16334 \\
\hline Southeastern dump site & J16B45 & J16B46 \\
\hline Northwestern dump site & J16DV7 & J16DV6 \\
\hline
\end{tabular}

Field duplicate samples are collected to provide a relative measure of the degree of local heterogeneity in the sampling medium, unlike laboratory duplicates that are used to evaluate precision in the analytical process. The field duplicates are evaluated by computing the RPD of the duplicate samples for each COC. The 95\% upper confidence limit (UCL) calculation brief in Appendix C provides details on duplicate pair evaluation and RPD calculation. Only analytes with values above five times the detection limits for both the main and duplicate samples are compared. None of the RPDs calculated exceeded the acceptance criteria of $30 \%$.

RPDs for the remaining analytes are not calculated because an evaluation of the data shows the analytes are not detected in both the main and duplicate sample at more than 5 times the target detection limit. RPDs of analytes detected at low concentrations (less than five times the detection limit) are not considered to be indicative of the analytical system performance. The data are useable for decision making purposes.

A secondary check of the data variability is used when one or both of the samples being evaluated (main and duplicate) is less than 5 times the target detection limit (TDL), including undetected analytes. In these cases, a control limit of \pm 2 times the TDL is used (Appendix C) to indicate that a visual check of the data is required by the reviewer. For the southern tank footprint focused duplicate sample, the difference was less than 2 times the TDL (for all analytes with one or both of the samples less than 5 times the TDL), and did not required the visual check. However, a visual inspection of all of the data is also performed. No additional major or minor deficiencies are noted. The data are useable for decision-making purposes.

\section{Summary}

Limited, random, or sample matrix-specific influenced batch quality control (QC) issues such as those discussed above, are a potential for any analysis. The number and types seen in these data sets are within expectations for the matrix types and analyses performed. The DQA review of the 120-F-1 verification sampling data found that the analytical results are accurate within the standard errors associated with the analytical methods, sampling, and sample handling. The DQA review for 120-F-1 waste site concludes that the reviewed data are of the right type, quality, and quantity to support the intended use. Detection limits, precision, accuracy, and sampling data group completeness were assessed to determine if any analytical results should be rejected as a result of QA and QC deficiencies. The analytical data were found acceptable for decision-making purposes. The verification sample analytical data are stored in the Environmental Restoration (ENRE) project-specific database prior to being submitted for 
inclusion in the Hanford Environmental Information System (HEIS) database. The verification sample analytical data are also summarized in Appendix C.

\section{REFERENCES}

BHI, 2000, Data Validation Procedure for Chemical Analysis, BHI-01435, Rev. 0, Bechtel Hanford, Inc., Richland, Washington.

DOE-RL, 2005a, 100 Area Remedial Action Sampling and Analysis Plan, DOE/RL-96-22, Rev. 4, U.S. Department of Energy, Richland Operations Office, Richland, Washington.

DOE-RL, 2005b, Remedial Design Report/Remedial Action Work Plan for the 100 Area, DOE/RL-96-17, Rev. 5, U.S. Department of Energy, Richland Operations Office, Richland, Washington.

EPA, 2000, Guidance for Data Quality Assessment, EPA QA/G-9, QA00 Update,

U.S. Environmental Protection Agency, Office of Environmental Information, Washington, D.C.

WCH, 2007, Work Instruction for Verification Sampling of the 120-F-1 Glass Dump Waste Site, Work Instruction No. 0100F-WI-G0069, Rev 0, Washington Closure Hanford, Richland, Washington.

WCH, 2008a, Revised 120-F-1 Verification Sampling, CCN 139173 dated February 14, 2008, to R. Lobos and D.C. Smith from J.M. Capron, Washington Closure Hanford, Richland, Washington.

WCH, 2008b, 100 F Remedial Sampling, Logbook EFL-1173-4 pp 37-39, 81-82, and 88-89, Washington Closure Hanford, Richland, Washington. 